\title{
Radical-Mediated C-H Functionalization: A Strategy for Access to Modified Cyclodextrins
}

\author{
Dimitri Alvarez-Dorta, ${ }^{\dagger}$ Elisa I. León,${ }^{\dagger}$ Alan R. Kennedy ${ }^{\ddagger}$ Angeles Martín, ${ }^{*}{ }^{\dagger}$ \\ Inés Pérez-Martín, ${ }^{\dagger}$ and Ernesto Suárez ${ }^{*}, \dagger$
}

†Síntesis de Productos Naturales, Instituto de Productos Naturales y Agrobiología del CSIC

Carretera de La Esperanza 3, 38206, La Laguna, Tenerife (Spain)

WestCHEM Department of Pure and Applied Chemistry University of Strathclyde, 295 Cathedral Street, Glasgow G1 1XL, Scotland (UK)

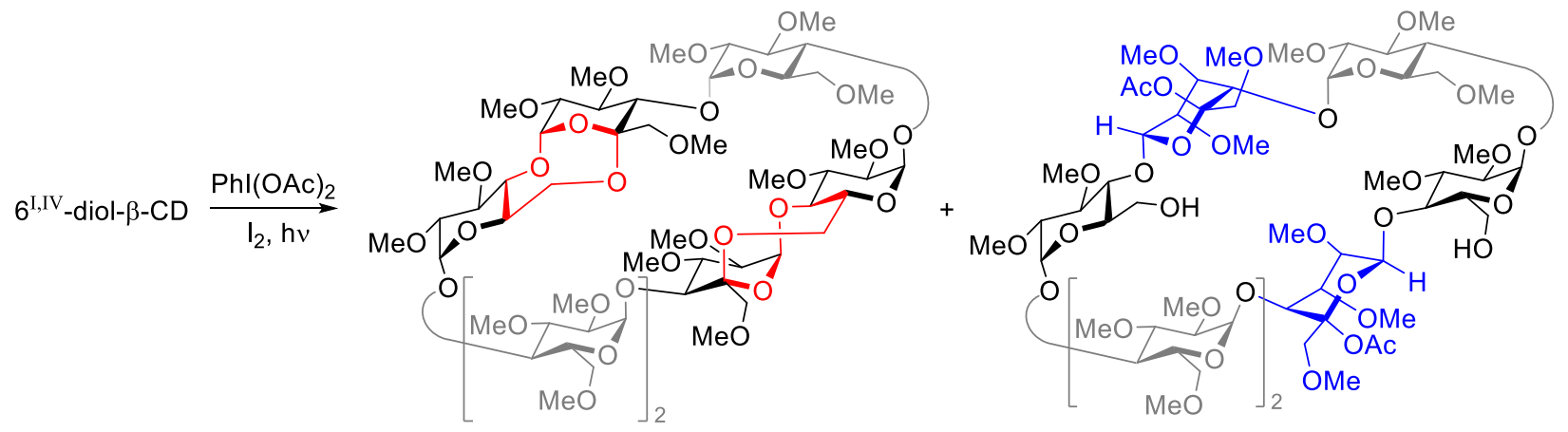

\section{ABSTRACT}

A simple and efficient radical $\mathrm{C}-\mathrm{H}$ functionalization to access modified cyclodextrins (CDs) has been developed. The well-defined conformation of glycosidic and aglyconic bonds in $\alpha-, \beta-$, and $\gamma$-CDs favors the intramolecular 1,8-hydrogen atom transfer (HAT) promoted by the $6^{\mathrm{I}}-O$-yl radical which abstracts regioselectively the hydrogen at $\mathrm{C} 5^{\mathrm{II}}$ of the contiguous pyranose. The $\mathrm{C} 5^{\mathrm{II}}$-radical evolves by a polar crossover mechanism to a stable 1,3,5-trioxocane ring between two adjacent 
glucoses or alternatively triggers the inversion of one $\alpha$-D-glucose into a 5 - $C$-acetoxy- $\beta$-L-idose unit possessing a ${ }^{1} C_{4}$ conformation. The $6^{\mathrm{IIV}}$ - and $6^{\mathrm{I}, \mathrm{IIII}}$-diols of $\alpha$ - and $\beta$-CDs behave similarly to the monoalcohols, forming mostly compounds originating from two 1,8-HAT consecutive processes. In the case of $6^{\mathrm{I}, \mathrm{II}}$-diols the proximity of the two 6-O-yl radicals in adjacent sugar units allows the formation of unique lactone rings within the $\mathrm{CD}$ framework via a 1,8 -HAT- $\beta$-scission tandem mechanism. X-Ray diffraction carried out on the crystalline 1,4-bis(trioxocane)- $\alpha$-CD derivative shows a severe distortion toward a narrower elliptical shape for the primary face.

\section{INTRODUCTION}

Over the past decades, cyclodextrins (CDs) have been extensively investigated for their valuable applications in several areas. ${ }^{1}$ These naturally-occurring cyclic oligosaccharides are composed of glucose units in ${ }^{4} C_{1}$ chair conformations, with a hydrogen bond network formed between the secondary hydroxyl groups, creating a conical shape with an internal hydrophobic cavity, conferring the ability to form host-guest or inclusion complexes with a great variety of molecules and rendering them one of the most important supramolecular host families. Because of these inclusion properties, CDs are displayed as being potentially useful as drug and gene delivery systems, ${ }^{2}$ artificial enzymes ${ }^{3}$ or catalysts, ${ }^{4}$ just to cite a few.

Considering their unique features and relevant applications, great effort has been invested in developing chemical modifications of native CDs to modulate their chemophysical properties, and thus achieve an enhancement of their solubility, a change in their cavity size or an improvement in their inclusion capability. The strategies are usually based on grafting a wide assortment of substituents taking into account the different accessibility and reactivity of the hydroxyl groups to 
obtain diverse patterns of functionalization, which act as platforms to access amphiphilic $\mathrm{CDs},{ }^{5}$ and hence, sophisticated molecular architectures. ${ }^{6}$

It should be pointed out that most of these methodologies have addressed the structural changes retaining the ${ }^{4} C_{1}$ chair conformation of the glucose units, but a few of them describe modifications of the cavity by altering the conformations of these units. Thus, the reported synthesis of 3,6anhydro-CDs shows the conformational change of the pyranose units to ${ }^{1} C_{4}$, and consequently, a significant change in their binding properties. ${ }^{7}$ Moreover, the formation of a mono-2,3-epoxide- $\beta$ CD followed by a nucleophilic ring opening generates an altrose unit in a ${ }^{1} C_{4}$ which distorts the cavity from conical to elliptical. ${ }^{8}$ The introduction of several altrose moieties reflects a rapid equilibrium between ${ }^{1} C_{4}$ and ${ }^{4} C_{1}$ conformers and their ratio depends on the number of residues and their position in the macromolecule. ${ }^{9}$ The cycloglycosylation syntheses of cyclodextrin analogues composed exclusively of L-sugars or incorporating both L- and D-sugars residues have been reported. ${ }^{7 a}$ Finally, it has been found that in the crystalline structure of permethylated $\beta$-CD one of the D-glucose residues is inverted from the normal ${ }^{4} C_{1}$ to the ${ }^{1} C_{4}$ chair conformation. ${ }^{10}$

Interestingly, in the methodologies employed to create this structural diversity in CDs, regardless of conformational changes, radical reactions are practically unknown and this fact is not surprising if we consider that in simple carbohydrates they mainly involve the anomeric position which is committed in the cyclic CD structure. ${ }^{11}$ Only one example has been reported in the literature and is focused on the intermolecular attack of reactive-oxygen-centered free radicals on CDs in aqueous solution using EPR detection experiments. ${ }^{12}$

During the last several years, our group has been immersed in the development of methods for the activation and functionalization of "inert" $\mathrm{C}-\mathrm{H}$ bonds in carbohydrate chemistry. ${ }^{13}$ Of special 
interest is a novel 1,8-hydrogen atom transfer reaction $(1,8$-HAT) which takes place, through a rather unusual nine-membered transition state (TS), between both pyranose units in a Hexp-(1 $\rightarrow 4)$ Hexp disaccharide system. The hydrogen abstraction at $\mathrm{C}^{\mathrm{II}}$ is promoted, in a highly regioselective manner, by the electrophilic $6^{\mathrm{I}}-O$-yl radical I generated from the corresponding alcohol under oxidative conditions, producing a C5 ${ }^{\mathrm{II}}$ radical II (Scheme 1).

Scheme 1. Radical polar crossover mechanism for the HAT reaction promoted by 6-O-yl radicals in Hexp-(1 $\rightarrow 4)$-Hexp systems of carbohydrates. DIB = $($ diacetoxyiodo $)$ benzene.
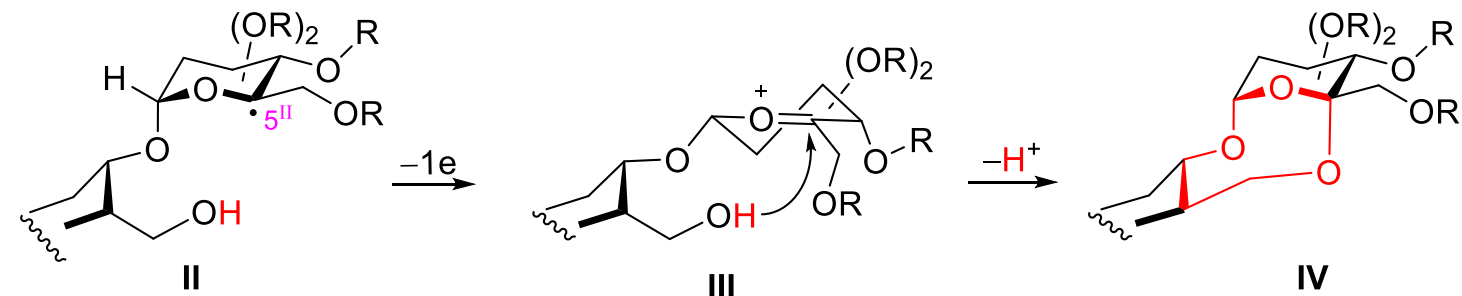

III

IV
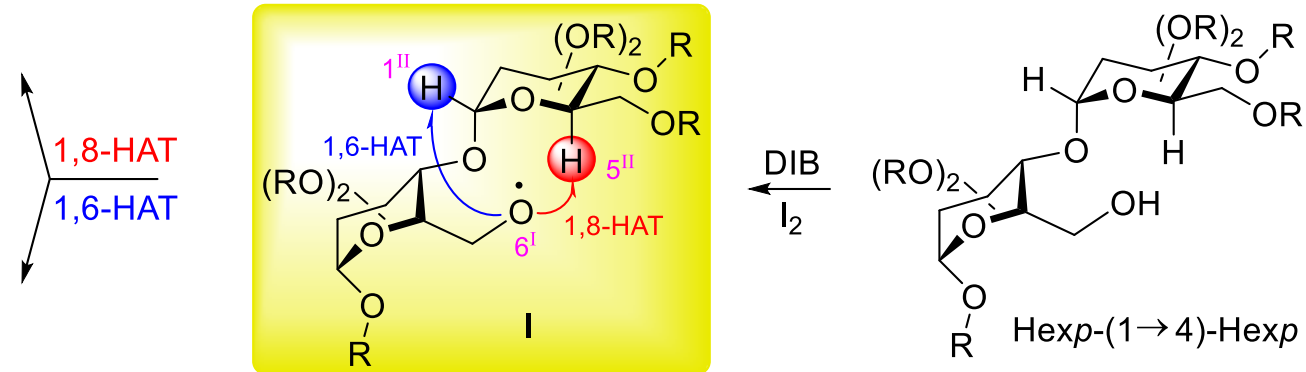

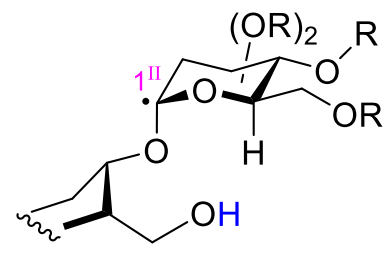

V

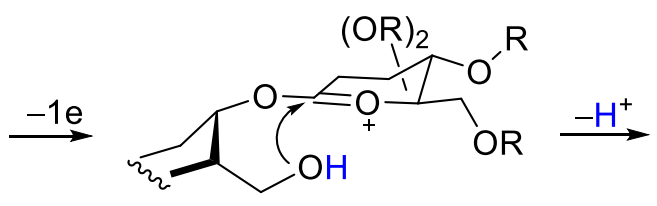

VI

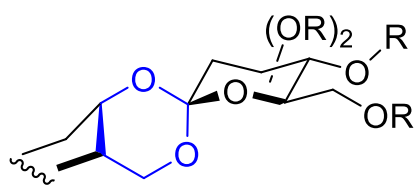

VII

$$
\mathrm{R}=\mathrm{Me}, \mathrm{Ac}
$$

This intermediate suffers a one-electron oxidation causing a crossover from the radical to the oxacarbenium ion III which is subsequently trapped by the alcohol to give the intramolecular bridged eight-membered 1,3,5-trioxocane IV. The obtained results show that this regioselectivity is highly dependent on both the four stereogenic centers implicated in the cyclization step $\left(\mathrm{C} 5^{\mathrm{II}}\right.$, 
$\mathrm{C} 1^{\mathrm{II}}, \mathrm{C} 4^{\mathrm{I}}$, and $\left.\mathrm{C} 5^{\mathrm{I}}\right)$ as the conformation of the glycosidic $\left(\Phi=\mathrm{H} 1^{\mathrm{II}}-\mathrm{C} 1^{\mathrm{II}}-\mathrm{O} 4^{\mathrm{I}}-\mathrm{C} 4^{\mathrm{I}}\right)$ and aglyconic $\left(\Psi=\mathrm{C} 1^{\mathrm{II}}-\mathrm{O} 4^{\mathrm{I}}-\mathrm{C} 4^{\mathrm{I}}-\mathrm{H} 4^{\mathrm{I}}\right)$ bonds. In fact, when these stereochemical and conformational requisites are not suitable for the 1,8-HAT to take place, the alkoxyl radical I may alternatively abstract the hydrogen at $\mathrm{C} 1^{\mathrm{II}}$ via a $1,6-\mathrm{HAT}$ to produce a new $\mathrm{C} 1^{\mathrm{II}}$ radical $\mathbf{V}$ which, after oxidation to the respective oxacarbenium ion VI and cyclization, gives the spiro ortho ester VII.

For the $\beta$-maltose disaccharide, $\alpha$-D-Glcp- $(1 \rightarrow 4)-\beta$-D-Glc $p$, the reaction proceed with complete regioselectivity, yielding compounds formed exclusively from the 1,8 -HAT. ${ }^{13 \mathrm{a}}$ In this case, the corresponding glycosidic and aglyconic bonds in the TS involved $\left(\Phi=-32.7, \Psi=-37.3^{\circ}\right)$ are arranged in an exo-syn conformation, where the alkoxyl radical and the $\mathrm{H} 5^{\mathrm{II}}$ are at an optimal distance of $3.1 \AA$ for the initial abstraction step. ${ }^{14}$

With this result in mind, we decided to investigate whether this radical-polar crossover methodology could be extended to more complex carbohydrates such as CDs, since in these

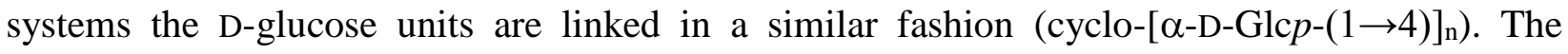
measures of distances and angles made on the reported X-ray structure of permethylated $\beta-C D^{15}$ as well as on its minimized structure indicate that there is sufficient inter-residue flexibility in the macromolecule to adopt an exo-syn conformation similar to $\beta$-maltose. Therefore, from a theoretical point of view, the 1,8-HAT process could take place between two vicinal units of the macrosystem to provide modified CDs on their primary face, which could exhibit important distortions in their cavities.

Preliminary results of this work have been described recently and we now present here the full details of the process and the extension to other positional isomeric diols and per-6-ol models. ${ }^{16}$ 


\section{RESULTS AND DISCUSSIONS}

Initially, the most common and readily available $\beta$-cyclodextrin was the substrate of choice in order to investigate the viability of this radical process. The corresponding monoalcohol $2^{\mathrm{I}-\mathrm{VII}}, 3^{\mathrm{I}-\mathrm{VII}}, 6^{\mathrm{II}-}$ VII-icosa- $O$-methyl- $\beta$-CD (1) necessary to assay the reaction was prepared from the commercial $\beta$ CD following a well-known three-step sequence consisting in monosilylation of one primary alcohol, $O$-methylation of the remaining hydroxyl groups and finally removal of silyl-ether. ${ }^{17}$ This protocol has the advantage of using methyl ethers as protecting groups which usually allow an increase in solubility both in water and in organic solvent. ${ }^{18}$ To our delight, visible light irradiation of alcohol 1 with diacetoxyiodobenzene (DIB) (2.2 equiv) and iodine (1 equiv) at $30^{\circ} \mathrm{C}$ proceeded nicely in 30 minutes delivering the expected 1,3,5-trioxocane $\mathbf{2}(36 \%)$ and the acetate $\mathbf{3}(61 \%)$ as the major compound (Table 1, entry 1 ). ${ }^{19}$ Lower reaction temperatures or fewer equivalents of reagents (DIB or $\mathrm{I}_{2}$ ) only gave rise to slower and incomplete reactions while the increase in the reaction times or the amount of reagents allowed us to obtain exclusively trioxocane $\mathbf{2}$ in $60 \%$ yield (entry 2). The reaction is plausibly explained by a sequential intermolecular nucleophilic attack by acetate anion to give $\mathbf{3}$ followed by an intramolecular cyclization leading to $\mathbf{2}$. A competitive addition of both nucleophiles to the oxacarbenium ion intermediate may also be considered. ${ }^{20}$

Obviously, both products, $\mathbf{2}$ and $\mathbf{3}$, derived from the same $\mathrm{C} 5^{\mathrm{VII}}$ radical intermediate, through an intramolecular 1,8-HAT reaction promoted by the $6^{\mathrm{I}}-O$-yl radical. No compounds resulting from abstraction at $\mathrm{C} 1^{\mathrm{VII}}$ via a 1,6-HAT process were detected.

In both compounds, the oxidation at $\mathrm{C}^{\mathrm{VII}}$ was clearly confirmed by 1D and 2D NMR experiments. Thus, for compound 2 , the HMBC correlation of the $\mathrm{H} 1{ }^{\mathrm{VII}}$ proton signal at $\delta_{\mathrm{H}} 5.06 \mathrm{ppm}$ with the $\mathrm{C}^{\mathrm{VIII}}$ quaternary carbon atom $\left(\delta_{\mathrm{C}} 101.04 \mathrm{ppm}\right)$ corroborates this $\mathrm{C} 5^{\mathrm{VII}}$ functionalization. Moreover, 
all D-glucopyranose units are preferentially in ${ }^{4} C_{1}$ chair conformations as we can be deduced from the values of the coupling constants of the anomeric hydrogens $\left({ }^{3} J_{1,2} \approx 3.6 \mathrm{~Hz}\right)$.

Table 1. 1,8-HAT for $6^{\mathrm{I}}-0 \mathrm{l}-\beta-\mathrm{CD} 1,6^{\mathrm{I}}-\mathrm{ol}-\alpha-\mathrm{CD} 5$, and $6^{\mathrm{I}}-\mathrm{ol}-\gamma-\mathrm{CD} 8 .^{a}$

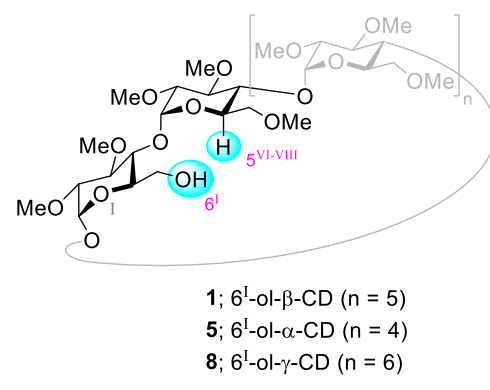

entry
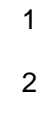

3

4

5

6

7

8

9

10

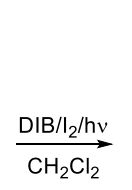

substrate

$1 ; \beta-C D$

$1 ; \beta-C D$

$1 ; \beta-C D$

$1 ; \beta-C D$

$1 ; \beta-C D$

$1 ; \beta-C D$

5; $\alpha-C D$

5; $\alpha-C D$

8; $\gamma-C D$

8; $\gamma-C D$

$\underset{\mathrm{CH}_{2} \mathrm{Cl}_{2}}{\stackrel{\mathrm{DIB} / \mathrm{I}_{2} / \mathrm{hv}}{\longrightarrow}}$ $\mathrm{MeO}$

$2(\mathrm{n}=5)$

$6(n=4)$

$9(n=6)$

$\mathrm{I}(\mathrm{III}) / \mathrm{I}_{2}$ (equiv)

$\mathrm{DIB} / \mathrm{I}_{2}(2.2 / 1.0)$

$\mathrm{DIB} / \mathrm{I}_{2}(3.0 / 1.7)$

$\mathrm{Phl}(\mathrm{OCOAd})_{2} / \mathrm{I}_{2}(2.2 / 1.0)^{b}$

$\mathrm{Phl}\left(\mathrm{OCO}-2,4,6-\mathrm{Pr}_{3} \mathrm{Ph}\right)_{2} / \mathrm{I}_{2}(2.2 / 1.0)$

$\mathrm{Phl}\left(\mathrm{OCO}-4-\mathrm{BuPh}_{2} / \mathrm{I}_{2}(2.2 / 1.0)\right.$

$\mathrm{Phl}\left(\mathrm{OCO}-2,4,6-\mathrm{Me}_{3} \mathrm{Ph}\right)_{2} / \mathrm{I}_{2}(2.2 / 1.0)$

$\mathrm{DIB} / \mathrm{I}_{2}(2.2 / 0.5)$

$\mathrm{DIB} / \mathrm{I}_{2}(2.2 / 1.0)$

$\mathrm{DIB} / \mathrm{I}_{2}(2.2 / 0.5)$

$\mathrm{DIB} / \mathrm{I}_{2}(2.2 / 1.0)$

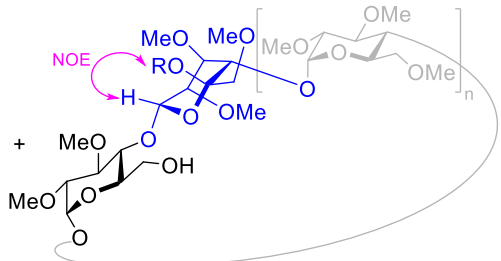

$3,4(n=5)$

$7(n=4)$

$10(n=6)$

products (yield)

$2(36 \%)$

$2(60 \%)$

No reaction

No reaction

$2(46 \%)$

n.d.

$2(21 \%)$

4; $\mathrm{R}=\mathrm{CO}-2,4,6-\mathrm{Me}_{3} \mathrm{Ph}(33 \%)$

$6(31 \%)$

$7 ; R=A c(30 \%)$

$6(59 \%)$

$9(36 \%)$

10; $R=A c(41 \%)$

9 (44\%)

3; $R=A c(61 \%)$

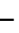

${ }^{a}$ Reaction conditions: A solution of the alcohol in dry $\mathrm{CH}_{2} \mathrm{Cl}_{2}$ containing the organoiodine(III) reagent and $\mathrm{I}_{2}$ was stirred under nitrogen while irradiated with two $80 \mathrm{~W}$ tungsten-filament lamps. $\mathrm{n} . \mathrm{d}$. $=$ not determined. ${ }^{b} \mathrm{Ad}=1$-Adamantyl.

With respect to the acetyl derivative $\mathbf{3}$, the deshielding observed for the ring protons of the unit VII bearing the acetyl group and the magnitude of the ${ }^{3} J_{\mathrm{H}, \mathrm{H}}$ coupling constants $\left[{ }^{3} J_{1,2}=1.9 \mathrm{~Hz}\right.$ (calcd $1.3 \mathrm{~Hz}),{ }^{3} J_{2,3}=2.5 \mathrm{~Hz}(\mathrm{calcd} 2.6 \mathrm{~Hz})$, and ${ }^{3} J_{3,4}=1.9 \mathrm{~Hz}($ calcd $\left.3.3 \mathrm{~Hz})\right]$ suggest that the ring exists substantially in a ${ }^{1} C_{4}$ inverted chair conformation. ${ }^{21}$ Moreover, the NOE experiment shows an interaction between $\mathrm{H} 1^{\mathrm{VII}}$ and the acetyl methyl protons, thus tentatively establishing the stereochemistry at $\mathrm{C} 5^{\mathrm{VII}}$ as $R$. These data indicate that the acetate group has been incorporated with 
inversion of configuration at $\mathrm{C} 5^{\mathrm{VII}}$ and consequently the original $\alpha$-D-glucopyranose has been transformed into a 5-C-acetoxy- $\beta$-L-idopyranose unit, ${ }^{22}$ flipping from ${ }^{4} C_{1}$ to ${ }^{1} C_{4}$ chair conformation. As far as we know, this is the first precedent of a $\beta$-CD molecule having a $\beta$-L-idose unit in its structure. Conversely, in the formation of trioxocane 2, the intramolecular nucleophilic addition to the oxacarbenium ion occurs with retention of configuration at $\mathrm{C} 5{ }^{\mathrm{VII}}$ or by a double inversion if we consider $\mathbf{3}$ to be an intermediate.

To achieve additional insight into the incorporation of acetate, we decided to explore the reaction with different bis(acyloxyl) hypervalent iodine(III) reagents to evaluate the nucleophilic addition of other acyloxyl groups to the C5 position. ${ }^{23}$ As depicted in Table 1, in the cases of the sterically demanding 1-adamantylcarboxyl (entry 3) or 2,4,6-triisopropylbenzoyloxyl (entry 4), the reaction did not proceed, recovering most of the unchanged starting material in both cases. This may be caused by the steric hindrance of the acyl hypoiodite intermediate which precludes entirely the formation of the 6 - $O$-yl radical. ${ }^{24}$ When the ester was the 4-tert-butylbenzoyloxyl (entry 5), TLC analysis showed the completion of the reaction and the presence of two main products. However after chromatographic separation we were able to isolate only the cyclized compound $\mathbf{2}$ in $46 \%$ yield, presumably due to intramolecular hydrolytic cleavage of the ester. Only the reaction with bis(2,4,6-trimethylbenzoyloxy)iodobenzene allowed us to isolate both products, $\mathbf{2}$ and $\mathbf{4}$, albeit in moderate yield, $21 \%$ and $33 \%$ respectively, together with a significant amount of starting material (39\%) (entry 6). The NMR data for $\mathbf{4}$ show clearly that, also in this case, the ring chair conformation of unit VII has been inverted.

The effectiveness of this methodology was tested on $\alpha$ - and $\gamma$-CD models. The corresponding monoalcohols $5^{25}$ and $\mathbf{8}^{26}$ were prepared following the same three-step protocol employed for the $6^{\mathrm{I}}$-ol- $\beta-\mathrm{CD}$ derivative 1. Using similar amounts of DIB and iodine, both substrates behaved in an 
analogous manner to the $\beta-\mathrm{CD}$ case, generating the corresponding trioxocane derivatives 6 and 9 and acetates 7 and 10, albeit in somewhat lower overall yields (Table 1, entries 7-10). While the trioxocane derivatives are stable for long periods of time at room temperature and can be easily manipulated, the acetates seem to be acid- and base-sensitive compounds. The use of an excess of iodine (entries 8 and 10), aqueous work up or standard silica gel column chromatographic purification preclude their isolation and the reaction gives exclusively the respective trioxocane derivatives $\mathbf{6}$ and $\mathbf{9}$ in variable yields. Fortunately, pure acetates $\mathbf{7}$ and $\mathbf{1 0}$ could be obtained using 0.5 equiv of iodine, avoiding any work up and after careful flash chromatography employing silica gel scraped from commercially coated TLC plates (entries 7 and 9).

As described above for the $\beta$-CD derivative 3, the structures and conformations of the acetyl derivatives $\mathbf{7}$ and $\mathbf{1 0}$ were corroborated by 1D TOCSY and 2D HSQC and HMBC experiments. Remarkably, the conformation of unit VI of compound 7 exhibits changes in the TOCSY experiments by varying from $\mathrm{CDCl}_{3}$ to $\mathrm{C}_{6} \mathrm{D}_{6}$. The observed values of the coupling constants of this unit in $\mathrm{CDCl}_{3}\left[{ }^{3} J_{1,2}=2.8 \mathrm{~Hz}(\right.$ calcd $1.4 \mathrm{~Hz}),{ }^{3} J_{2,3}=3.2 \mathrm{~Hz}($ calcd $2.5 \mathrm{~Hz})$, and ${ }^{3} J_{3,4}=2.8 \mathrm{~Hz}($ calcd $3.3 \mathrm{~Hz})]$ indicate predominantly a ${ }^{1} C_{4}$ conformation while in $\mathrm{C}_{6} \mathrm{D}_{6}$ these values $\left[{ }^{3} J_{1,2}=3.5 \mathrm{~Hz}\right.$ (calcd $3.2 \mathrm{~Hz}$ ), ${ }^{3} J_{2,3}=8.5 \mathrm{~Hz}$ (calcd $9.7 \mathrm{~Hz}$ ), and ${ }^{3} J_{3,4}=7.9 \mathrm{~Hz}$ (calcd $9.2 \mathrm{~Hz}$ )] preferentially point to a ${ }^{4} C_{1}$ conformation. ${ }^{21}$ In contrast, the acetates 3,4 , and 10 , derived from $\beta$ - and $\gamma-\mathrm{CD}$, show the same ${ }^{1} C_{4}$ conformation in both solvents. The inversion barrier seems to be significantly lower in the more strained hexameric ring.

Encouraged by these promising results with mono-alcohols, we decided to investigate what would happen in this HAT process if we have two primary hydroxyl groups in the molecule which can react simultaneously, a situation hitherto little studied. ${ }^{27}$ Taking into account the structure of $\beta$ - and $\alpha$-CDs, there are three different positional isomers $\left(6^{\mathrm{I}, \mathrm{II}}-6^{\mathrm{I}, \mathrm{III}}-\right.$ and $6^{\mathrm{I}, \mathrm{IV}}-$ diol $)$ for each of them. 
With the aim of obtaining cyclized compounds, we examined the relative stability of the expected final products, the 1,4-, 1,3-, and 1,2-bis(1,3,5-trioxocane) compounds. A preliminary analysis using molecular mechanic calculations revealed that the energetic differences among the three isomers, in either $\beta$ - or $\alpha-\mathrm{CD}$, are very small (approx. $2 \mathrm{kcal} / \mathrm{mol}$ ), providing evidence for the thermodynamic feasibility of the cyclization step. ${ }^{28}$

Therefore, we decided to synthesize the three isomeric diols of each CD using a similar protectiondeprotection sequence employed for the monoalcohols, as has been described previously, ${ }^{17 a}$ and assess the scope of this methodology.

The study was begun with the more distant 1,4-diol derivatives, that we believe have greater possibilities of success. Firstly, we carried out the reaction of the $6^{\mathrm{IIV}}-$ diol- $\beta-\mathrm{CD}$ derivative $11^{17 \mathrm{a}}$ with DIB and $I_{2}$ as shown in Scheme 2. Gratifyingly, the process proceeded in good overall yield, affording the 1,4-bis(trioxocane) $\mathbf{1 2}(20 \%)$, the diacetate $\mathbf{1 3}(49 \%)$ as the main product, and a 3:2 chromatographically inseparable mixture of mono-trioxocane-mono-acetyl positional isomers (14\%, not shown, see the Experimental Section) which could be transformed into 12 after treatment with iodine. Analogously, compound $\mathbf{1 2}$ was obtained when $\mathbf{1 3}$ was submitted to these iodine catalyzed conditions. The acetyl methyl protons at $\mathrm{C}^{\mathrm{III}}$ and $\mathrm{C} 5^{\mathrm{VII}}$ in compound $\mathbf{1 3}$ were readily assigned by 2D HSQC and HMBC experiments, whereas the ${ }^{1} \mathrm{H}$ NMR showed the deshielding of the ring protons in these units III and VII and the values of their coupling constants $\left({ }^{3} J_{\mathrm{H}, \mathrm{H}} \approx 1.9-\right.$ $2.5 \mathrm{~Hz}$ ) were consistent with the introduction of both acetates with inversion at $\mathrm{C} 5$ and thus, with the presence of two $\beta$-L-idose units in ${ }^{1} C_{4}$ conformations. Although no chair inversion was detected using $\mathrm{C}_{6} \mathrm{D}_{6}$ as solvent, the axial $\mathrm{H} 1{ }^{\mathrm{III}}$ and $\mathrm{H} 1{ }^{\mathrm{VII}}$ protons started to broaden at $18^{\circ} \mathrm{C}$ suggesting that coalescence is just below this temperature, while the signals were sharpened by heating at $70{ }^{\circ} \mathrm{C}$. Additionally, the stereochemistry of the quaternary centers $\mathrm{C}^{\mathrm{III}}$ and $\mathrm{C} 5^{\mathrm{VII}}$ were established 
tentatively as $R$ on the basis of NOE interactions between $\mathrm{H} 1^{\mathrm{III}}$ or $\mathrm{H} 1^{\mathrm{VII}}$ and the respective acetyl methyl protons.

\section{Scheme 2. 1,8-HAT for the $6^{\mathrm{I}, \mathrm{IV}}$-diol- $\beta$-CD 11 and $6^{\mathrm{I}, \mathrm{IV}}$-diol- $\alpha-C D 14$}

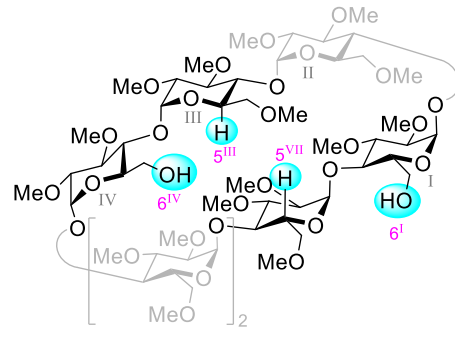

$11 ; 6^{\mathrm{I}, \mathrm{IV}}$-diol- $\beta-\mathrm{CD}$

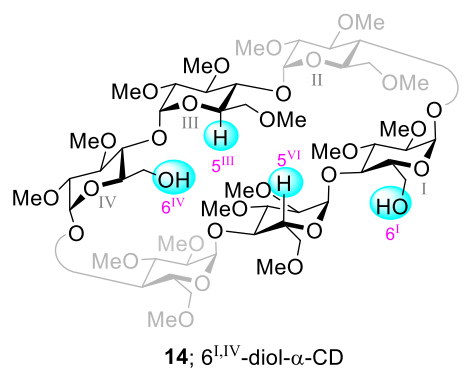

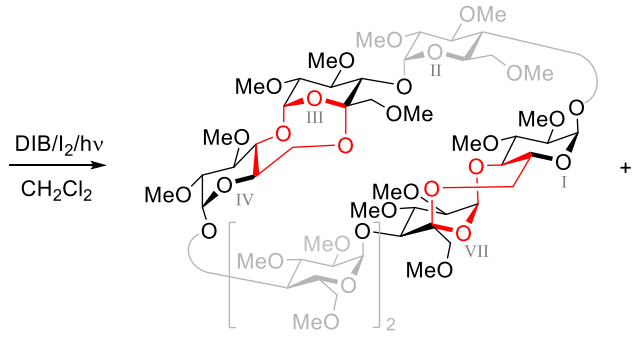

$12(20 \%)$

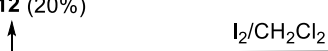

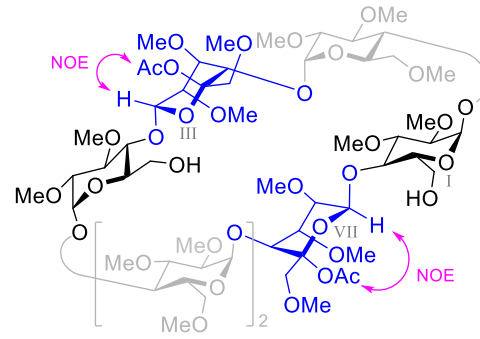

$13(49 \%)$
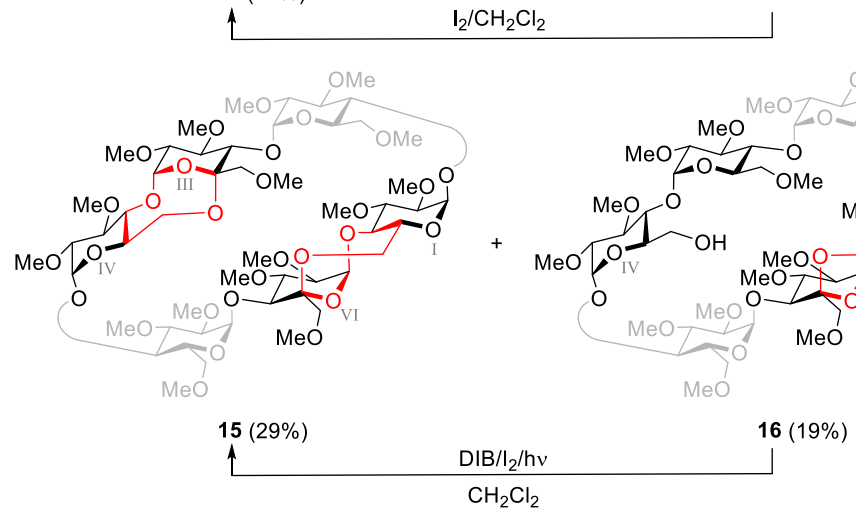

Next, we explored the behavior of $6^{\mathrm{IIV}}$-diol- $\alpha$-CD derivative $14^{25,29}$ under these oxidative radical conditions. The reaction afforded the bis- and mono-trioxocanes $\mathbf{1 5}$ and $\mathbf{1 6}$ as sole compounds, not detecting any product from incorporation of acetate into the molecule, and thus, revealing the greater steric hindrance in this hexamer (Scheme 2). Moreover, compound $\mathbf{1 5}$ is a crystalline solid whose structure was unambiguously confirmed by X-ray crystallographic analysis, showing two trioxocane rings in a restricted boat-chair (BC) conformation with a guest $n$-hexane molecule in the CD cavity (Figure 1). ${ }^{30}$ The simplified ${ }^{1} \mathrm{H}$ and ${ }^{13} \mathrm{C}$ NMR spectra are consistent with the $C_{2}$ symmetry of the proposed structure.

The comparison of the geometrical structural parameters of $\mathbf{1 5}$ with those of the related permethylated $\alpha-C D^{31}$ shows that the presence of the two 1,3,5-trioxocane rings severely distort 
the primary face of the molecule. This distortion is clearly observed when comparing the geometrical parameters of the irregular hexagon comprising the side-chain carbon atoms $\left(\mathrm{C}^{\mathrm{n}}\right)$, which points toward a much narrower elliptical shape for this rim (see Tables S2 and S3 in the Supporting Information). Conversely, the presence of the bis(trioxocane) rings does not significantly alter the secondary face of the molecule. Thus, for instance, the distances and angles between the six interglycosidic oxygen atoms $\left(\mathrm{O}^{\mathrm{n}}\right)$, as well as the radii of the gravity center of the hexagon formed by these $\mathrm{O}^{\mathrm{n}}$ oxygen atoms are quite similar in both X-ray structures. The most significant differences are observed in the tilt angles made by the $\mathrm{O} 4^{\mathrm{n}}$ mean plane and the mean planes through the glucose units principally in residues II and V adjacent to the trioxocane rings. What do not seem to be affected either are the ${ }^{4} C_{1}$ chair geometries of the six glucose units, as shown by the Cremer-Pople puckering parameters which describe slightly distorted chairs similar to those found in the permethylated $\alpha-\mathrm{CD}$ (see Tables S2 and S3). ${ }^{32}$

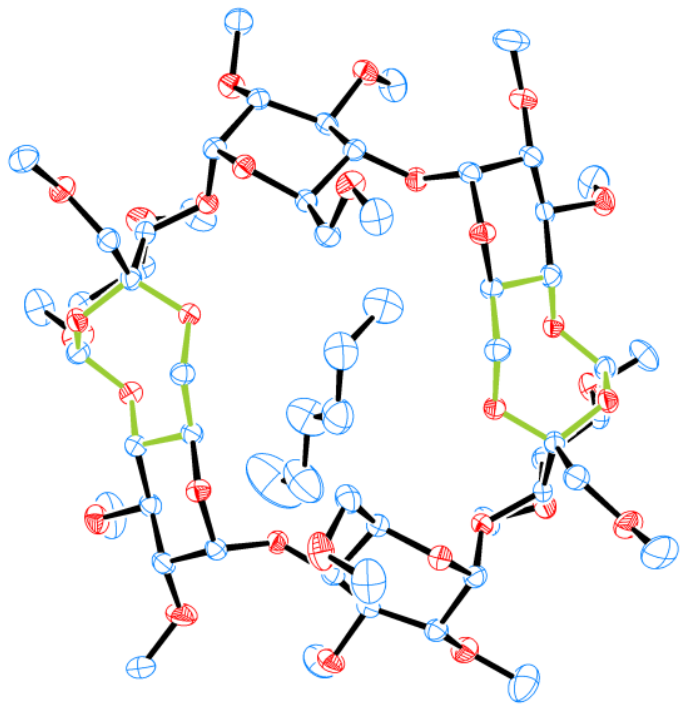

Figure 1. X-ray crystal structure of 15. C blue, O red, 1,3,5-trioxocane ring yellow-green, $\mathrm{H}$ atoms have been omitted by clarity. Ellipsoids are set at $50 \%$ probability. 


\section{Scheme 3. HAT for the $6^{\mathrm{I}, \mathrm{III}}$-diol- $\beta-\mathrm{CD} 17$}

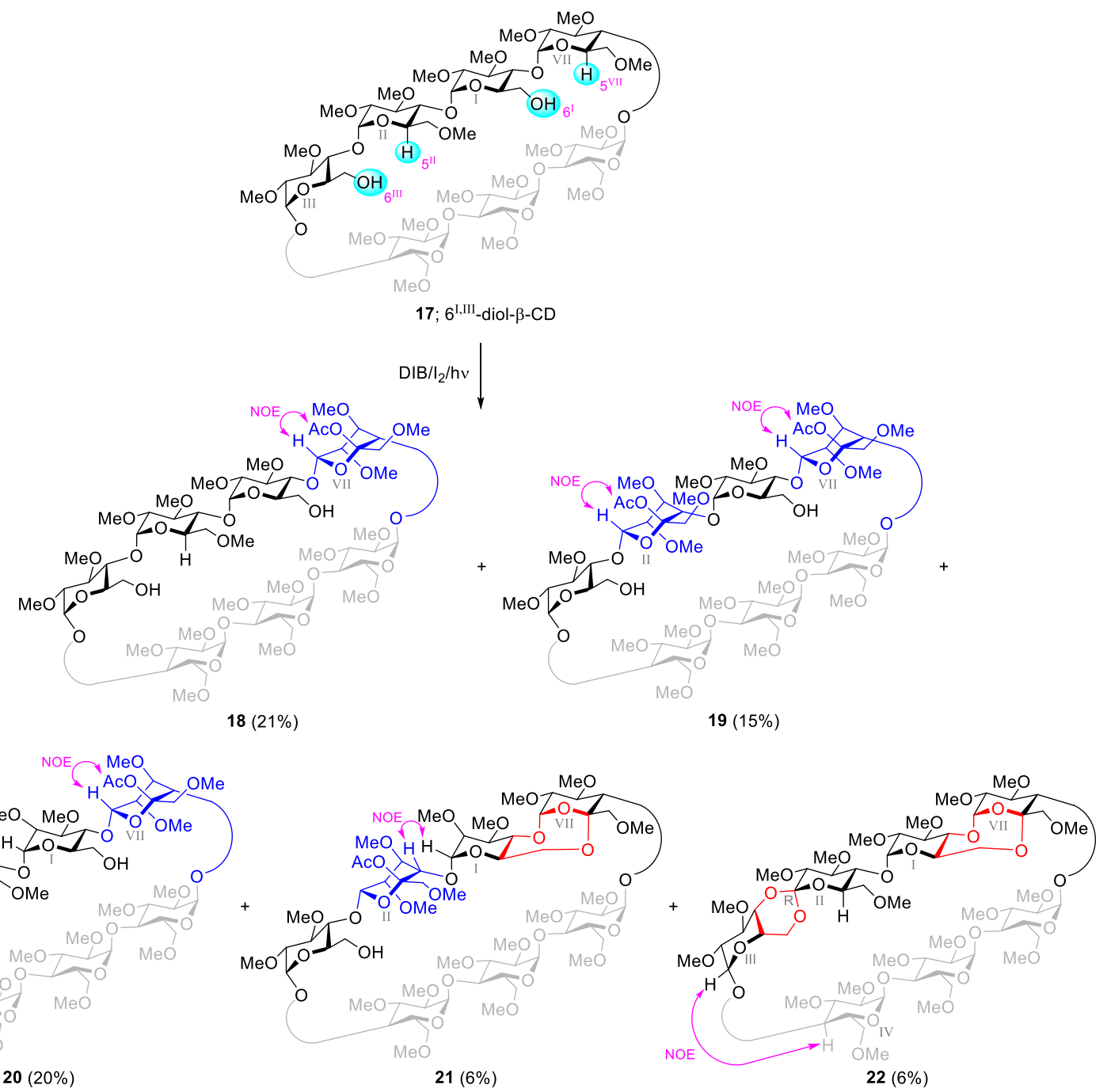

We next focused our attention on applying the $\mathrm{C}-\mathrm{H}$ functionalization to 1,3-diol derivatives. The reaction of $6^{\mathrm{I}, \mathrm{III}}$-diol- $\beta$-CD $17^{17 \mathrm{a}}$ under the usual conditions produced one compound derived from the mono-abstraction, the acetate $\mathbf{1 8}$ in $21 \%$ yield together with four compounds in $47 \%$ overall yield arising from double abstraction: the diacetate 19 with incorporation of two external nucleophiles, the two possible mono-trioxocane-mono-acetyl derivatives $\mathbf{2 0}$ and $\mathbf{2 1}$, and the spiro ortho ester-trioxocane $\mathbf{2 2}$ in which only internal nucleophiles are implicated (Scheme 3). 
Curiously, the expected bis(trioxocane) was not detected in the crude reaction mixture, even after longer reaction times.

Analogously, the incorporation of the acetate has taken place with inversion at C5 and transformation of the sugar into a $\beta$-L-idose unit. This appears to be a general trend for all compounds even in the cases of compounds $\mathbf{1 3}$ and $\mathbf{1 9}$ where two units in a ${ }^{1} C_{4}$ inverted chair conformation exist as part of the heptameric ring.

On the other hand, the positional isomers $\mathbf{2 0}$ and $\mathbf{2 1}$ can be differentiated by a careful study using 2D HSQC and HMBC and 1D TOCSY and ROESY experiments. The H1 $1^{\mathrm{I}}$ and H4 ${ }^{\mathrm{II}}$ 1D ROESY correlation that has been observed in both compounds can be used to distinguish between both isomers. The $\mathrm{H} 4{ }^{\mathrm{II}}$ protons, that have been clearly identified by $1 \mathrm{D}$ TOCSY, appear at $3.90 \mathrm{ppm}(\mathrm{d}$,

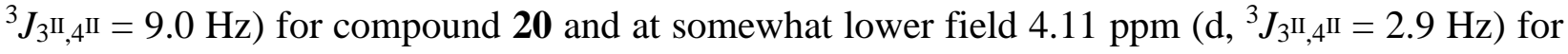
the isomer 21, which undoubtedly confirms the proposed structures.

Moreover, in compound $\mathbf{2 2}$ where two positional isomers could be a priori possible we were able to assign the correct structure, since the 1D ROESY experiment showed a correlation between $\mathrm{H} 1^{\mathrm{III}}$ and $\mathrm{H}^{\mathrm{IV}}[(3.60 \mathrm{ppm}(\mathrm{dd}, J=9.2,9.2 \mathrm{~Hz})]$. In the other hypothetical isomer (spiro ortho ester at unit I and trioxocane at unit III) the analogous ROESY interaction would have been between $\mathrm{H} 1^{\mathrm{I}}$ and $\mathrm{H} 4^{\mathrm{II}}$ near the trioxocane and this last proton should be a doublet. Although two stereoisomers at the spiro quaternary carbon at $\mathrm{C}^{\mathrm{II}}$ are possible, only one has been isolated and the configuration tentatively assigned as $R$. Molecular mechanics calculations show that the $R$-isomer is more stable by $6 \mathrm{kcal} / \mathrm{mol}$ than the $S$-isomer. The stereochemistry is supposed to be stereoelectronically controlled, in the $R$-isomer the alcohol approaches the oxacarbenium ion by the $\alpha$-axial direction to maximize the anomeric effect. ${ }^{33}$ Additional support was secured by examination of the ${ }^{1} \mathrm{H}$ NMR spectrum, revealing a downfield displacement for the pro-S H6 ${ }^{\mathrm{III}}$ proton $[4.24(\mathrm{dd}, J=9.1,7.9$ 
$\mathrm{Hz})]$, which in this stereoisomer presents a 1,3 -diaxial interaction with the $\mathrm{C} 1^{\mathrm{II}}-\mathrm{O}^{\mathrm{II}}$ bond. An obvious experiment that could be important for the determination of this stereochemistry such as the study of NOE correlations from $\mathrm{H} 2^{\mathrm{II}}$ could not be performed due to signal overlap. Fortunately, this problem does not exist in the ${ }^{1} \mathrm{H}$ NMR spectrum of the analogous spiro ortho ester $\mathbf{3 0}$ and the results confirm the proposed stereochemistry (vide infra).

In contrast with the previous results, when the reaction is performed with the hitherto unknown $6^{\mathrm{I}, \mathrm{III}}$-diol- $\alpha-\mathrm{CD} 23$, we only obtained the bis- and the two possible mono-trioxocanes $\mathbf{2 4 ,} 25$ and 26 respectively, in $66 \%$ overall yield, not detecting products with inverted $\beta$-L-idose units in the molecule (Scheme 4). This is in agreement with the results obtained in the $6^{\mathrm{I}, \mathrm{IV}}$-diol series where we did not find inverted sugar units in the reaction of $6^{\mathrm{I}, \mathrm{IV}}-$ diol- $\alpha-\mathrm{CD} 14$ (Scheme 2).

\section{Scheme 4. HAT for the $6^{\mathrm{I}, \mathrm{III}}-$ diol- $\alpha-\mathrm{CD} 23$}

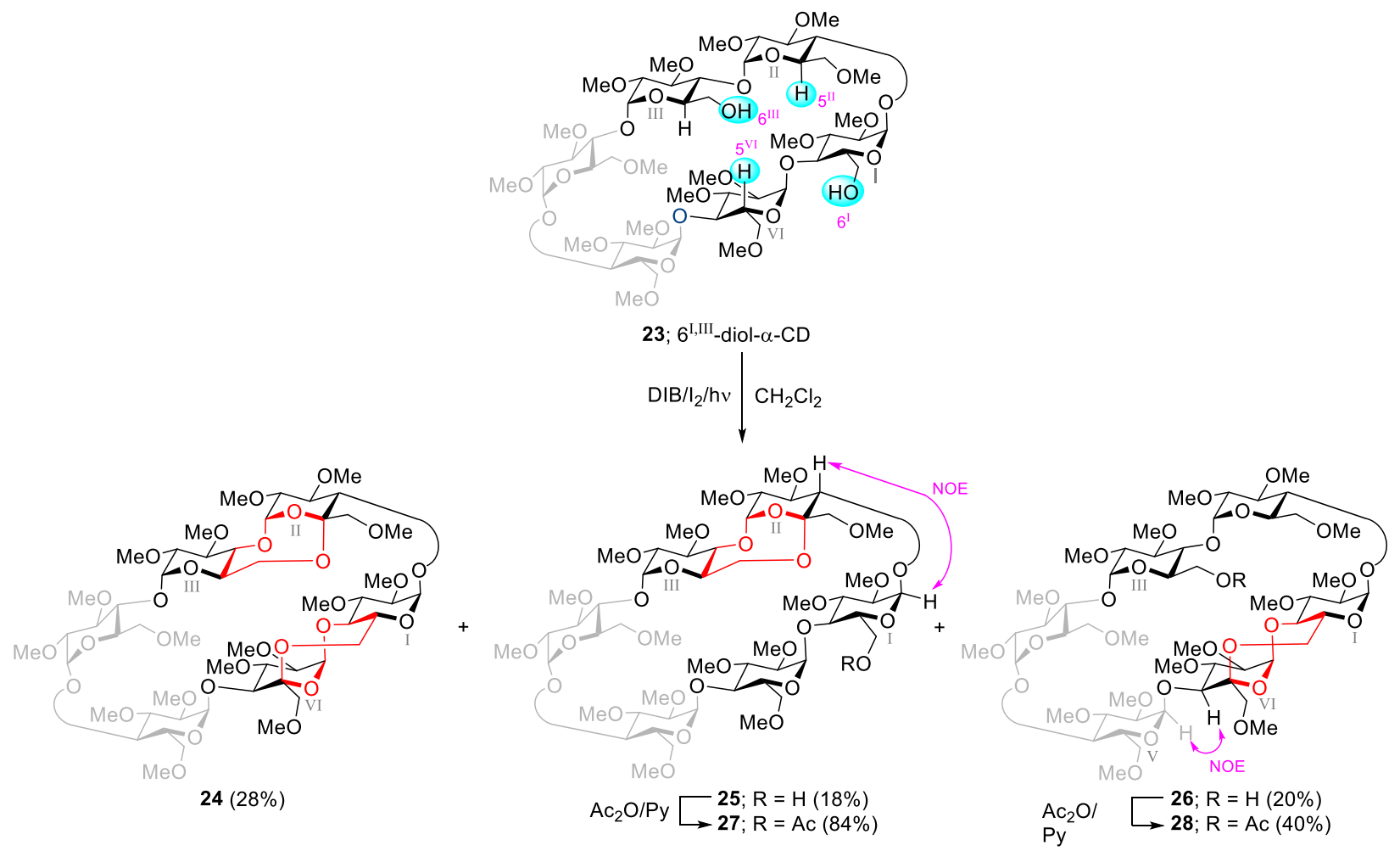


Although, all structures were analogously determined by NMR spectroscopy the preparation of the acetyl derivatives $\mathbf{2 7}$ and $\mathbf{2 8}$ from both mono-trioxocanes was necessary to differentiate these positional isomers. The NOE interaction between $\mathrm{H} 1^{\mathrm{I}}$ and $\mathrm{H} 4^{\mathrm{II}}[4.26 \mathrm{ppm}(\mathrm{d}, J=9.2 \mathrm{~Hz})]$ in compound 27 and the corresponding correlation between $\mathrm{H} 4^{\mathrm{VI}}$ and $\mathrm{H} 1^{\mathrm{V}}$ [5.04 $\left.\mathrm{ppm}(\mathrm{d}, J=3.2 \mathrm{~Hz})\right]$ in the isomer $\mathbf{2 8}$ firmly stablished the proposed structures.

Finally, to complete the study of the diols, the reactions of 1,2-diols of $\beta$ - and $\alpha$-CDs were carried out. The reaction of the $6^{\mathrm{I}, \mathrm{II}}-$ diol $-\beta-\mathrm{CD} 29^{17 \mathrm{a}}$ under the usual oxidative conditions led to the formation of four compounds in moderate overall yield: the spiro ortho ester-trioxocane $\mathbf{3 0}(5 \%)$, the ten-membered lactone $\mathbf{3 1}(10 \%)$ and a mixture of two products, $\mathbf{3 2}$ and $\mathbf{3 3}$, which after acetylation was readily separated as the mono-trioxocane $\mathbf{3 4}$ and the $\delta$-lactone $\mathbf{3 5}$, in $22 \%$ and $11 \%$ yield respectively (Scheme 5). The relative position of the spiro ortho ester with respect to the trioxocane rings in $\mathbf{3 0}$ and the primary acetate with respect to the trioxocane in $\mathbf{3 4}$ was readily established by spectroscopic means. The ring coupling constants for the sugar units involved were determined by 1D TOCSY and ROESY experiments but in any case, the alternative structures for the positional isomers should have a very different ${ }^{1} \mathrm{H}$ and ${ }^{13} \mathrm{C}$ NMR pattern. The spiro quaternary carbon stereochemistry $\mathrm{C} 1^{\mathrm{I}}$ in compound $\mathbf{3 0}$ was assigned as $R$ on the basis discussed above for analogous spiro ortho ester 22 . The absence of NOE interactions between $\mathrm{H} 2^{\mathrm{I}}$ and the pro-S $\mathrm{H} 6^{\mathrm{II}}$, which was expected for the $S$-isomer, provides additional support for the anomeric configuration as $R$.

The ten-membered lactone $\mathbf{3 1}$ possesses a one-carbon dehomologated skeleton and among its more important structural and functional features are: the presence of carboxyl and acetal functions and the disappearance of one of the anomeric carbons of the starting $\beta$-CD. The protons of the sugar residues I and II can be differentiated by 1D TOCSY and all the above appears to confirm the 
decanolide structure proposed, while additional support is provided by HMBC 1,3-correlations, being of special significance that observed between the carboxyl carbon at $\mathrm{C}^{\mathrm{I}}$ and the two protons at $\mathrm{H}^{\mathrm{II}}$.

\section{Scheme 5. HAT for the $6^{\text {I,II-diol- } \beta-C D} 29$}
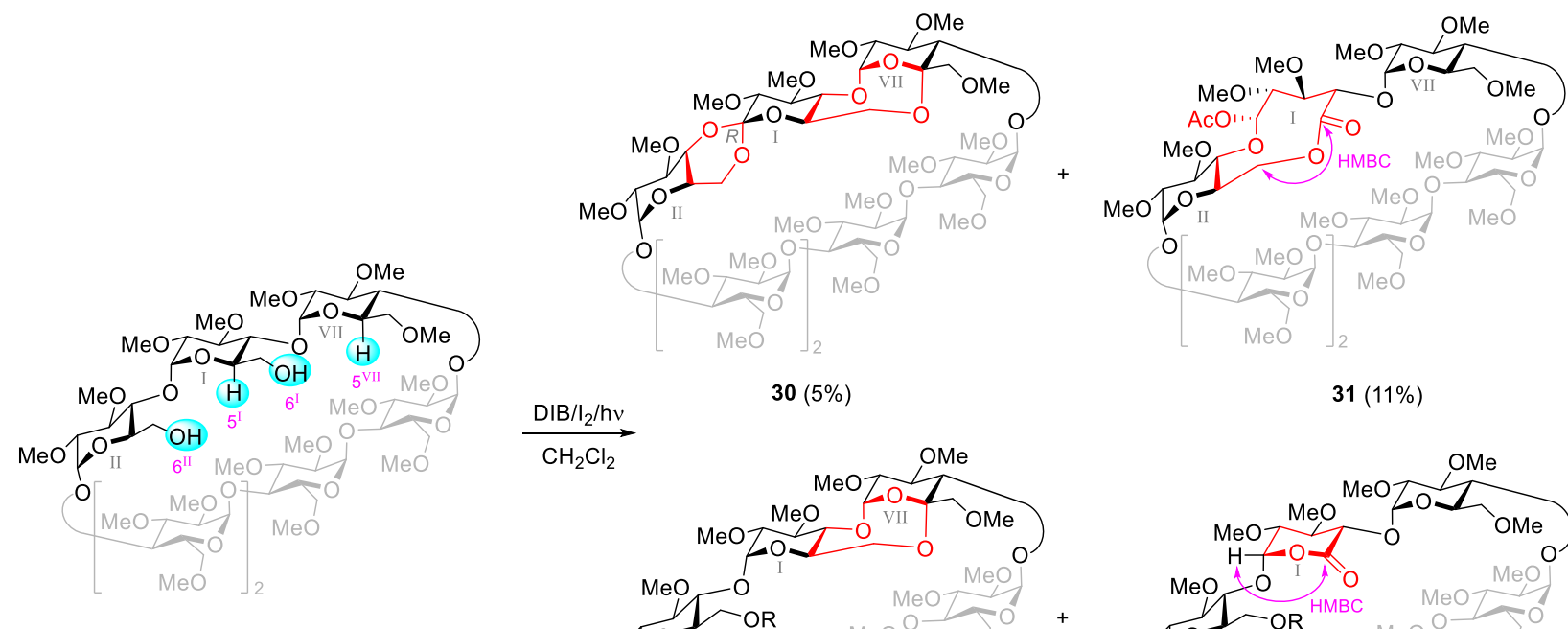

29; $6^{\text {I,II-diol }-\beta-C D}$

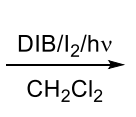

$30(5 \%)$

$31(11 \%)$
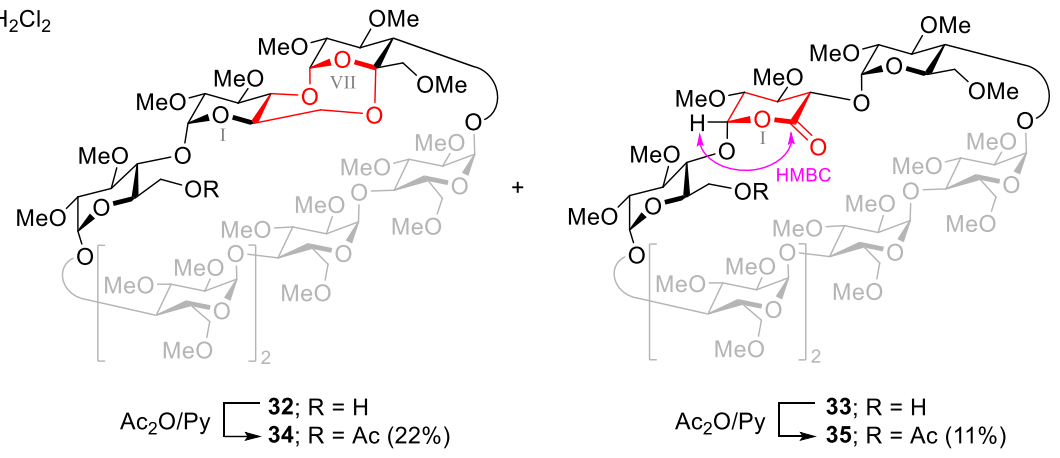

The $\delta$-lactone 35 has also a one carbon dehomologated skeleton and the more notable features of its structure are the large deshielding observed for the chemical shift of the anomeric hydrogen at $\mathrm{H}^{\mathrm{I}}[5.56 \mathrm{ppm}(\mathrm{d}, J=3.2 \mathrm{~Hz})]$ and the $1,3-\mathrm{HMBC}$ correlation of this proton with the carboxylic carbon at $\mathrm{C}^{\mathrm{I}}$.

According to these results, the formation of all products described in Scheme 5 could be explained in terms of which of the two primary alkoxyl radicals $\left(6^{\mathrm{I}}-O\right.$-yl or $6^{\mathrm{II}}-O$-yl) is generated first. In this sense, compound 30 should be formed by the $6^{\mathrm{I}}-O$-yl radical 1,8 -abstraction in the first place followed by a second 1,6-abstraction of the $6^{\mathrm{II}}-O$-yl radical to generate the spiro ortho ester. Clearly, the primary alcohol $\mathbf{3 2}$ should be an intermediate in the formation of $\mathbf{3 0}$. On the other 
hand, the mechanism for the formation of lactones $\mathbf{3 1}$ and $\mathbf{3 3}$ from diol $\mathbf{2 9}$ certainly implies a prior abstraction of hydrogen at $\mathrm{H} 5^{\mathrm{I}}$ by the $6^{\mathrm{II}}-\mathrm{O}$-yl radical to give the trioxocane VIII (Scheme 6 ). The excess of reagent generates a new $6^{\mathrm{I}}-\mathrm{O}$-yl radical from the free primary alcohol which by $\beta$-scission with loss of formaldehyde produces a $\mathrm{C} 5^{\mathrm{I}}$ tertiary radical IX ${ }^{34}$ This intermediate evolves following two competitive paths: (i) it may experience a second $\beta$-scission of the $\mathrm{C} 1^{\mathrm{I}}-\mathrm{O}^{\mathrm{I}}$ bond leading to the $\mathrm{C} 1{ }^{\mathrm{I}}$-radical $\mathbf{X}$, which by one-electron oxidation and nucleophilic attack by the acetate anion finally gives the ten-membered lactone $\mathbf{3 1}$ and (ii) it can be directly stabilized by oxidation-nucleophilic attack to give the $\delta$-lactone $\mathbf{3 3}$ previous hydrolysis of ortho ester intermediate XI. Neither intermediate VIII nor XI could be detected in the crude reaction mixture.

\section{Scheme 6. Proposed mechanistic pathway for the formation of lactones 31 and 33}

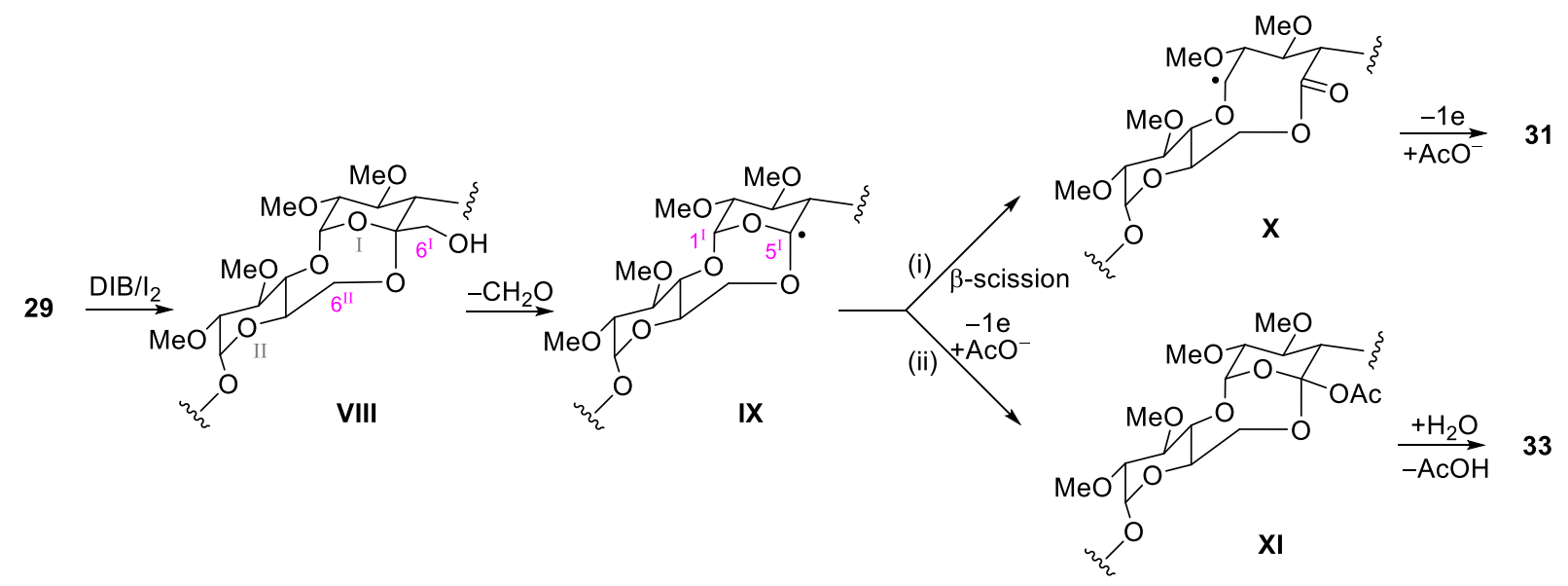

Analogously to the $\beta-\mathrm{CD}$ derivative, the $\mathrm{C}-\mathrm{H}$ functionalization of the $6^{\mathrm{I}, \mathrm{II}}$-diol- $\alpha-\mathrm{CD} \mathbf{3 6}^{35}$ proceeded in a similar way, giving the corresponding ten-membered lactone 37, the monotrioxocane 38 and the $\delta$-lactone 39 in $51 \%$ overall yield (Scheme 7). All these compounds show NMR features similar to those of the respective compounds 31, 34 and 35 and their formation can be explained by a mechanism identical with that described for the $\beta$-CD model (Scheme 6). 
Although, as commented previously, the formation of the 1,2-bis(trioxocane)s in $\alpha$ - and $\beta$-CDs may be thermodynamically possible, we have been unable to detect any of them. Therefore, the possibility of a second 1,8-HAT on the logical precursors, the isolated mono-trioxocanes $\mathbf{3 2}$ and 38, was studied by molecular mechanics. ${ }^{28}$ In the minimized structures of these compounds the distances between the $6^{\mathrm{II}}-\mathrm{O}$-yl radical and the extractable hydrogens at $\mathrm{H} 5^{\mathrm{I}}$ are excessively long (4.7 and $5.5 \AA$, respectively) and 1,8-HAT reactions should be clearly unfavorable (see Figure S2 and Table S4 in the SI for more details). Notwithstanding, the alternative 1,6-HAT of the H1 ${ }^{\mathrm{I}}$ appears possible in the case of compound $32\left(d \mathrm{O} 6^{\mathrm{II}}-\mathrm{H} 1^{\mathrm{I}}=2.7 \AA\right)$ that would explain the formation of spiro ortho ester $\mathbf{3 0}$ in the $\beta-\mathrm{CD}$ series. This $1,6-\mathrm{HAT}$ reaction is very unlikely in the $\alpha-\mathrm{CD}$ series, since in 38 a calculated distance $\mathrm{O}^{\mathrm{II}}-\mathrm{H} 1^{\mathrm{I}}$ of $4.6 \AA$ would prevent the formation of the corresponding spiro ortho ester.

\section{Scheme 7. HAT for the $6^{\text {I,II-diol- } \alpha \text {-CD } 36}$}

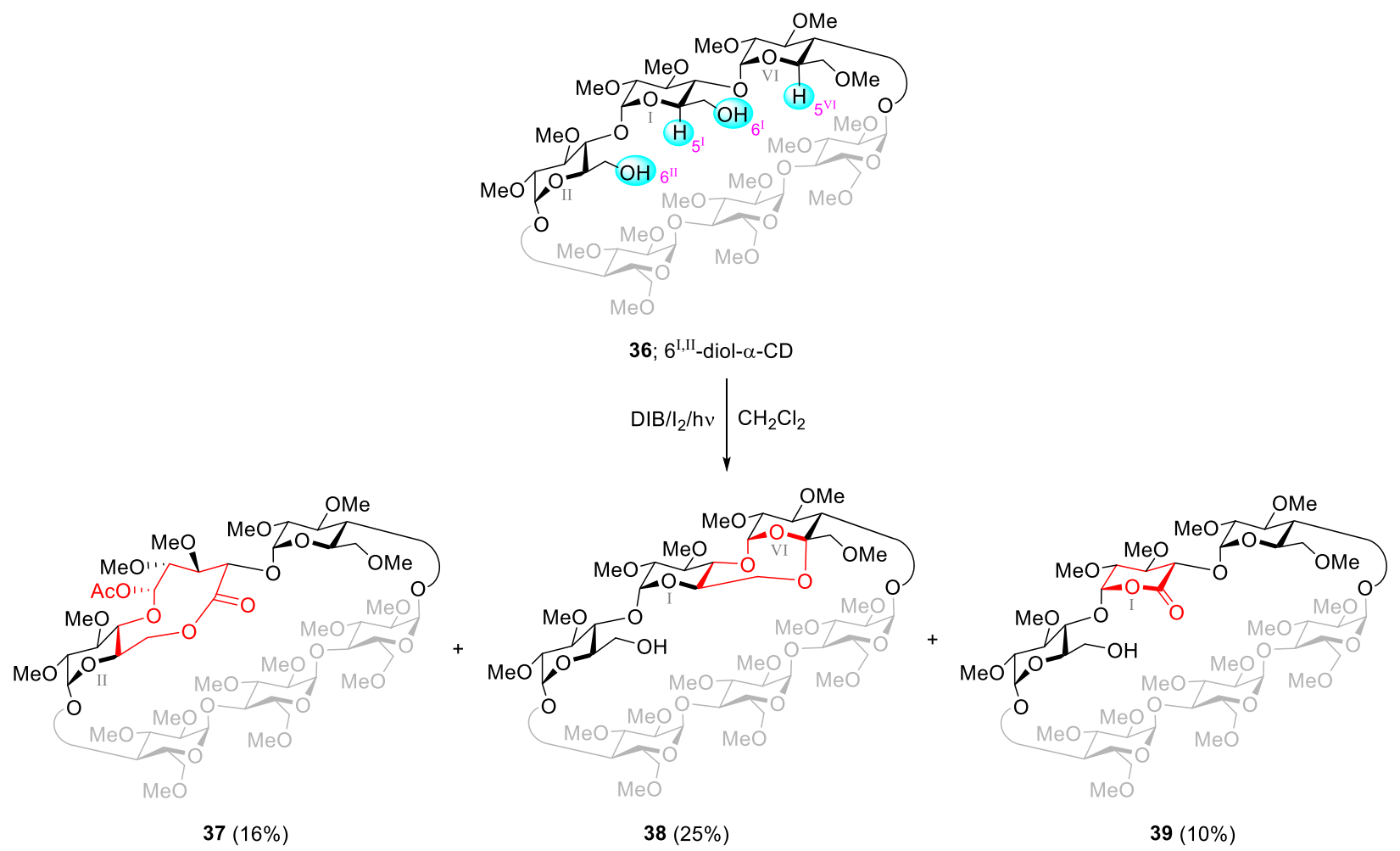


To gain insight into the reaction mechanism and clarify the different behavior of the $6^{\mathrm{I}, \mathrm{II}}$-diols in the $\mathrm{C}-\mathrm{H}$ functionalization with respect to the other positional isomers, we carried out the process with the corresponding diol in a more simple and conformationally flexible molecule such as the hexamethyl $\beta$-maltose derivative $\mathbf{4 1}$ obtained in three steps from $\mathrm{D}-(+)$-maltose through the silylether derivatives $40 \alpha$ and $40 \beta$ (Scheme 8). In this case, the reaction generated a complex mixture from which we were able to isolate the ten-membered lactone $\mathbf{4 2}$ together with compound $\mathbf{4 3}$ in only $20 \%$ overall yield. Both compounds were found to be unstable toward chromatographic purification. This instability does not prevent complete characterization but is presumably responsible for the small yield obtained.

\section{Scheme 8. HAT for the $6^{\mathrm{I}, \mathrm{II}}$-diol- $\beta$-maltose 41}

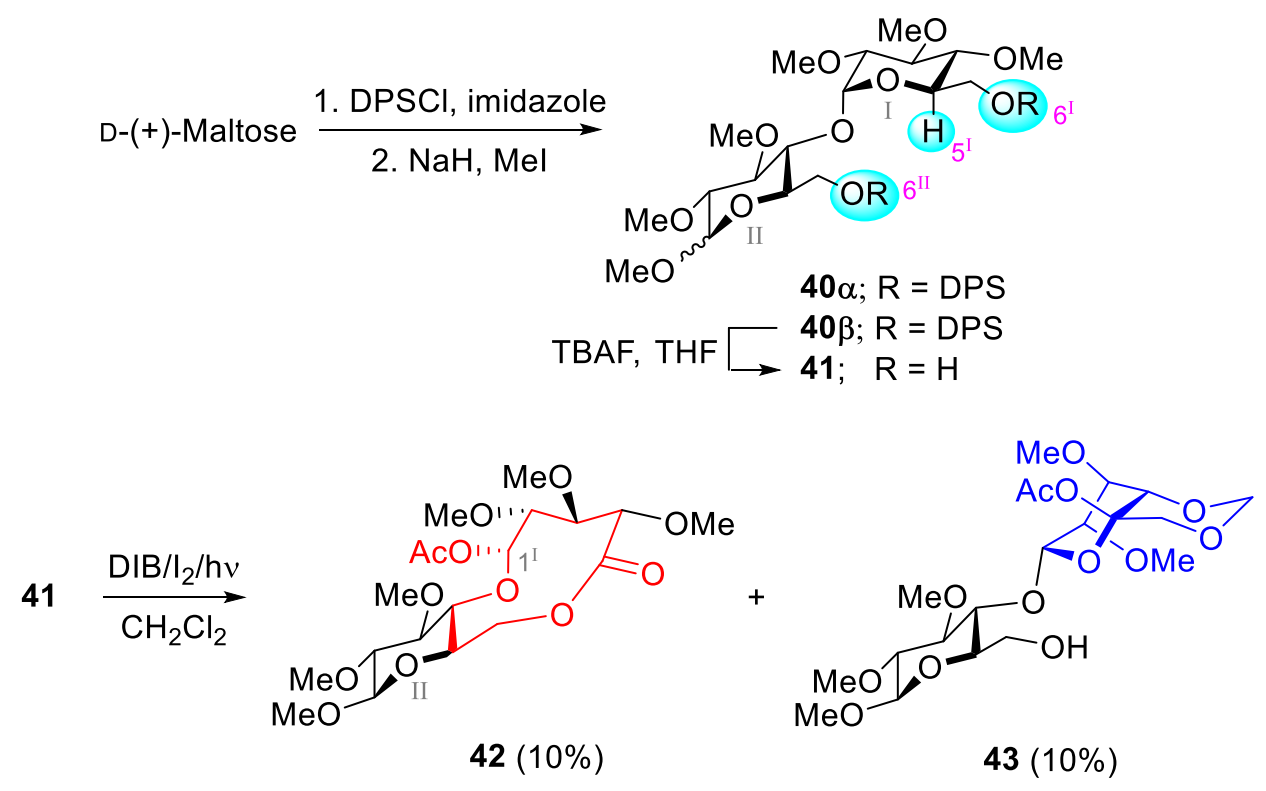

The structure and stereochemistry of the ten-membered lactone $\mathbf{4 2}$, where all ring protons are directly observable in the ${ }^{1} \mathrm{H}$ NMR spectrum, can be now more conveniently and unambiguously studied. The NMR data obtained are in quite good agreement with those previously described for the ten-membered lactones $\mathbf{3 1}$ and $\mathbf{3 7}$, which had been obtained mostly using 1D TOCSY. The 
presence of NOE interactions between $\mathrm{H} 1^{\mathrm{I}}$ and all the protons positioned on the $\beta$-face of the decanolide ring $\left(\mathrm{H} 2^{\mathrm{I}}, \mathrm{H} 4^{\mathrm{I}}, \mathrm{H} 4^{\mathrm{II}}\right.$, and pro-S $\left.\mathrm{H} 6^{\mathrm{II}}\right)$ seems to indicate a stereoselective $\alpha$-attack of the nucleophile and an $R$ configuration for the $\mathrm{C}^{\mathrm{I}}$. The relative position of the involved ring protons and the ${ }^{3} J_{\mathrm{H}, \mathrm{H}}$ coupling constants has been checked on a minimized structure where the tenmembered lactone adopts a boat-chair-chair (BCC) conformation with an $(R)$-configuration for the $\mathrm{C} 1^{\mathrm{I}} \cdot{ }^{36}$

While the decanolide formation could be explained by the mechanism shown above, the new disaccharide 43 could arise from initial 1,6-hydrogen abstraction by the $6^{\mathrm{I}}-\mathrm{O}$-yl radical from the proximal methoxyl group at $\mathrm{C} 4^{\mathrm{I}}$ followed by oxidation and cyclization to form a 1,3-dioxane ring. ${ }^{37}$ Then, a second 1,8 -abstraction now by the $6^{\mathrm{II}}-O$-yl radical generates a $\mathrm{C} 5^{\mathrm{I}}$-radical which finally collapses to the oxacarbenium ion and the incorporation of acetate with inversion of configuration. Also in this case, the coupling constants for the vicinal ring protons account for a preferred ${ }^{1} C_{4}$ chair conformation for the L-idose unit. The occurrence of this compound sheds some light on the mechanism, the less hindered environment permitting this second 1,8-abstraction which in neither case was observed when the more restricted $6^{\mathrm{I}, \mathrm{II}}$-diols of $\beta$ - and $\alpha$-CDs were used, namely compounds $\mathbf{2 9}$ (Scheme 5) and $\mathbf{3 6}$ (Scheme 7).

Until now, only mono- and di-alcohols have been investigated. We thought that this study would not be complete without trying the reaction with per-6-ol models derived from $\beta$ - and $\alpha$-CDs, $2^{\mathrm{I}-}$ ${ }_{\mathrm{VII}}, 3^{\mathrm{I}-\mathrm{VII}}$-tetradeca- $O$-methyl- $\beta$-cyclomaltoheptaose ${ }^{38,5 \mathrm{f}} \quad$ and $\quad 2^{\mathrm{I}-\mathrm{VI}}, 3^{\mathrm{I}-\mathrm{VI}}$-dodeca- $O$-methyl- $\alpha$ cyclomaltohexaose, ${ }^{39,5 f}$ to find out if we can access to polyfunctionalized compounds through multiple radical abstractions. In both substrates, several different conditions were screened in which the reagents stoichiometry, temperature, and time were varied, to no avail, only intractable 
mixtures of products being observed in all instances. Such a result is not very surprising if we consider the many possibilities of abstraction and the presumed instability of the products formed.

\section{CONCLUSIONS}

In summary, we have successfully applied for the first time the remote $\mathrm{C}-\mathrm{H}$ functionalization logic to $\mathrm{CD}$ systems. The process is initiated by the $6^{\mathrm{I}}-O$-yl radical which abstracts with complete regioselectivity the hydrogen atom at $\mathrm{C} 5^{\mathrm{II}}$ located in the adjacent $\mathrm{D}$-glucose by a favored geometrically restricted nine-membered transition state. Using this methodology with monoalcohols derived from $\alpha-, \beta$-, and $\gamma$-CDs two types of structurally modified CDs can be obtained in moderate to good yields: a) a new stable 1,3,5-trioxocane ring is formed between the two glucopyranose units involved and b) one of the ${ }^{4} C_{1}$ glucopyranose residues has been transformed into a ${ }^{1} C_{4} \beta$-L-idose unit by inversion of configuration at $\mathrm{C} 5^{\mathrm{II}}$. Interestingly, in the case of $\alpha$-CD derivative 7 , the conformation of the $\beta$-L-idose ring is solvent-dependent, and the ${ }^{1} C_{4}$ conformer is favored in $\mathrm{CDCl}_{3}$ while the equilibrium is strongly shifted toward the ${ }^{4} C_{1}$ chair in $\mathrm{C}_{6} \mathrm{D}_{6}$ solution. This phenomenon that can be readily detected at $26{ }^{\circ} \mathrm{C}$ by $1 \mathrm{D}$ TOCSY experiments is not observable in $\beta$ - and $\gamma$-CD analogs $\mathbf{3}, \mathbf{4}$, and $\mathbf{1 0} .^{40}$

This methodology was also applied to $6^{\mathrm{IIII}}-6^{\mathrm{I}, \mathrm{III}}-$, and $6^{\mathrm{IIV}}-$ diols of $\alpha$ - and $\beta$-CDs with the intention of assessing the viability of two hydrogen abstractions in a simultaneous or tandem fashion, a

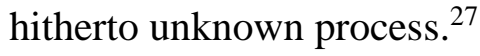

The $6^{\mathrm{IIV}}$-diol- $\beta-\mathrm{CD}$ derivative $\mathbf{1 1}$ behaved as if the two primary alcohols were independent, the expected products with two trioxocane rings $\mathbf{1 2}$ and two inverted $\beta$-L-idose units $\mathbf{1 3}$ being formed in good overall yield (Scheme 2). In the reaction of $6^{\mathrm{I}, \mathrm{IV}}$-diol- $\alpha-\mathrm{CD}$ derivative 14 a bis(trioxocane) 
15 was also formed. However, in this more restricted hexameric ring, the corresponding products with one or two $\beta$-L-idose units were not detected.

Among the products that can be generated by a double 1,8 -abstraction in the reaction of $6^{\mathrm{I}, \mathrm{III}}$-diol$\beta$-CD 17, three (19, 20 and 21) out of four possible have been obtained (Scheme 3). The only one that could not be isolated was the corresponding bis(trioxocane). Instead, the trioxocane-spiro ortho ester 22, formed also by a double abstraction, this time 1,8- followed by $1,6-\mathrm{HAT}$, was obtained. Unexpectedly, any attempt to cyclize the plausible intermediates 19-21 by acid catalysis failed to produce the bis(trioxocane). A mixture of chromatographically more polar unstable compounds presumably containing $\mathrm{CD}$ ring fragmentation products was obtained in all cases.

In sharp contrast to the situation encountered above with $6^{\mathrm{I}, \mathrm{III}}$-diol- $\beta$-CD 17 , the reaction of $6^{\mathrm{I}, \mathrm{III}}$ diol- $\alpha$-CD 23 led to the bis(trioxocane) 24 as the major product. (Scheme 4). Apart from the acid instability of the intermediates, we have no satisfactory explanation at the moment to account for the fact that the 1,3-bis(trioxocane) is easily formed in the $\alpha-\mathrm{CD}$ and not in the $\beta$-CD reaction.

In the case of the $6^{\mathrm{I}, \mathrm{II}}$-diol- of $\beta$ - and $\alpha$-CD, 29 and 36 respectively, the proximity of the two alkoxyl radicals in adjacent sugar units, facilitates the possibility of interaction between them. This situation which has not been presented in the previous diols is responsible for the formation of the ten-membered lactones ( $\mathbf{3 1}$ and $\mathbf{3 7}$ ) and the $\delta$-lactones ( $\mathbf{3 5}$ and $\mathbf{3 9})$. These compounds are obtained by the proposed mechanism (vide supra) where the two alkoxyl radicals are involved in the formation of the lactonic rings (Schemes 5, 6, and 7).

To the best of our knowledge, this is the first time that a radical protocol is applied to create structural differentiation on the primary face of CDs. Moreover, the mild reaction conditions and the good efficiency are remarkable features that make this process a powerful tool to access 
modified CDs and obtain macrocyclic rings with a different range of functionalization, otherwise difficult to achieve, and with potential synthetic and pharmaceutical applications.

\section{EXPERIMENTAL SECTION}

General Experimental Methods. Melting points were measured on a hot-stage apparatus. Optical rotations were recorded on a polarimeter at a wavelength of $589 \mathrm{~nm}$ at room temperature in $\mathrm{CHCl}_{3}$ solutions. IR spectra were recorded on a FT-IR spectrophotometer in film. ${ }^{1} \mathrm{H}$ NMR spectra were determined at $500 \mathrm{MHz}$ in $\mathrm{CDCl}_{3}$ or $\mathrm{C}_{6} \mathrm{D}_{6}$. Chemical shifts are reported in parts per million (ppm) and are calibrated to residual solvent peaks $\left(\mathrm{CHCl}_{3} 7.26 \mathrm{ppm}\right.$ and $\left.\mathrm{C}_{6} \mathrm{H}_{6} 7.15 \mathrm{ppm}\right) .{ }^{13} \mathrm{C}$ NMR spectra were determined at $125.7 \mathrm{MHz}$ in $\mathrm{CDCl}_{3}$ or $\mathrm{C}_{6} \mathrm{D}_{6}$. Chemical shifts are reported in parts per million (ppm) and are calibrated to residual solvent peaks $\left(\mathrm{CHCl}_{3} 77.0 \mathrm{ppm}\right.$ and $\left.\mathrm{C}_{6} \mathrm{H}_{6} 128.0 \mathrm{ppm}\right)$. NMR peaks assignments and stereochemistries have been established using COSY, TOCSY, DEPT, HMBC, HSQC, and ROESY experiments. Low and high resolution mass spectra were recorded with a TOF analyzer spectrometer by using electrospray $\left(\mathrm{ESI}^{+}\right)$. Flash column chromatography was performed on Merck silica gel $60 \mathrm{PF}(0.063-0.2 \mathrm{~mm})$. Sensitive compounds were purified by medium-pressure column chromatography using TLC silica gel $60 \mathrm{~F}_{254}$, scraped from Merck Millipore aluminum sheets (Product No. 1055540001), as adsorbent. Reaction progress was monitored by thin-layer chromatography (TLC) carried out on $0.25 \mathrm{~mm}$ coated commercial silica gel plates impregnated with a fluorescent indicator $(254 \mathrm{~nm})$. The spray reagents for TLC analysis were conducted with $0.5 \%$ vanillin in $\mathrm{H}_{2} \mathrm{SO}_{4}-\mathrm{EtOH}(4: 1)$ and further heating until development of color. Commercially available reagents and solvents were analytical grade or were purified by standard procedures prior to use.

Oxidative HAT of $2^{I-V I I}, 3^{I-V I I}, 6^{I I-V I I}$-icosa-O-methyl- $\beta$-cyclomaltoheptaose (1) with DIB and iodine. Method A: A solution of alcohol $\mathbf{1}^{17 \mathrm{~b}}(50 \mathrm{mg}, 0.035 \mathrm{mmol})$ in dry $\mathrm{CH}_{2} \mathrm{Cl}_{2}(2.4 \mathrm{~mL})$ 
containing DIB (33.4 mg, $0.105 \mathrm{mmol})$ and $\mathrm{I}_{2}(15.5 \mathrm{mg}, 0.06 \mathrm{mmol})$ was stirred under nitrogen at $30{ }^{\circ} \mathrm{C}$ for $0.75 \mathrm{~h}$ while irradiated with two $80 \mathrm{~W}$ tungsten-filament lamps. An excess of solid $\mathrm{Na}_{2} \mathrm{~S}_{2} \mathrm{O}_{3}$ was then added and stirring continued until complete disappearance of the iodine color. The reaction mixture was then filtered and concentrated under reduced pressure. Silica gel [Merck $60 \mathrm{PF}(0.063-0.2 \mathrm{~mm})]$ column chromatography of the reaction residue (hexanes-acetone, 65:35) afforded $\quad$ cyclo- $5^{\mathrm{VII}}, 6^{\mathrm{I}}$-anhydro-( $\left.5^{\mathrm{VII}} R\right)-(2,3,6$-tri- $O$-methyl- $\alpha$-D- $x y l o$-hexos-5-ulopyranosyl)$(1 \rightarrow 4)-2,3-$ di- $O$-methyl- $\alpha$-D-glucopyranosyl-[(1 $\rightarrow 4)-2,3,6$-tri- $O$-methyl- $\alpha$-D-glucopyranosyl $]_{5}$

(2) (30 mg, $0.021 \mathrm{mmol}, 60 \%)$ : colorless oil, $[\alpha]_{\mathrm{D}}+138.5$ (c 1.78, $\mathrm{CHCl}_{3}$ ); IR (film): 2929, 1143, 1107, $1042 \mathrm{~cm}^{-1} ;{ }^{1} \mathrm{H}$ NMR (500 MHz, $\left.\mathrm{CDCl}_{3}\right): \delta 3.365$ (s, 3H), 3.371 (s, 9H), $3.376(\mathrm{~s}, 3 \mathrm{H}), 3.384$ $(\mathrm{s}, 3 \mathrm{H}), 3.461(\mathrm{~s}, 3 \mathrm{H}), 3.465(\mathrm{~s}, 3 \mathrm{H}), 3.48(\mathrm{~s}, 3 \mathrm{H}), 3.49(\mathrm{~s}, 3 \mathrm{H}), 3.50(\mathrm{~s}, 6 \mathrm{H}), 3.51(\mathrm{~s}, 3 \mathrm{H}), 3.58(\mathrm{~s}$, 3H), $3.60(\mathrm{~s}, 3 \mathrm{H}), 3.62(\mathrm{~s}, 6 \mathrm{H}), 3.63(\mathrm{~s}, 3 \mathrm{H}), 3.64(\mathrm{~s}, 3 \mathrm{H}), 3.69(\mathrm{~s}, 3 \mathrm{H}), 5.06(\mathrm{~d}, J=3.5 \mathrm{~Hz}, 1 \mathrm{H})$, $5.06(\mathrm{~d}, J=3.5 \mathrm{~Hz}, 1 \mathrm{H}), 5.07(\mathrm{~d}, J=3.8 \mathrm{~Hz}, 1 \mathrm{H}), 5.08(\mathrm{~d}, J=3.2 \mathrm{~Hz}, 1 \mathrm{H}), 5.14(\mathrm{~d}, J=3.5 \mathrm{~Hz}$, $1 \mathrm{H}), 5.20(\mathrm{~d}, J=4.4 \mathrm{~Hz}, 1 \mathrm{H}), 5.21(\mathrm{~d}, J=3.8 \mathrm{~Hz}, 1 \mathrm{H}) ;{ }^{13} \mathrm{C} \mathrm{NMR}\left(125.7 \mathrm{MHz}, \mathrm{CDCl}_{3}\right): \delta 57.78$ $\left(\mathrm{CH}_{3}\right), 57.95\left(\mathrm{CH}_{3}\right), 58.04\left(\mathrm{CH}_{3}\right), 58.36\left(\mathrm{CH}_{3}\right), 58.51\left(\mathrm{CH}_{3}\right), 58.58\left(\mathrm{CH}_{3}\right), 58.86\left(3 \times \mathrm{CH}_{3}\right), 59.01$ $\left(\mathrm{CH}_{3}\right), 59.03\left(3 \times \mathrm{CH}_{3}\right), 60.91\left(\mathrm{CH}_{3}\right), 61.04\left(\mathrm{CH}_{3}\right), 61.26\left(\mathrm{CH}_{3}\right), 61.37\left(\mathrm{CH}_{3}\right), 61.65\left(2 \times \mathrm{CH}_{3}\right)$, $62.17\left(\mathrm{CH}_{3}\right), 64.81\left(\mathrm{CH}_{2}\right), 66.88(\mathrm{CH}), 70.74(\mathrm{CH}), 70.78(\mathrm{CH}), 70.90(\mathrm{CH}), 70.92\left(\mathrm{CH}_{2}\right), 70.96$ $(\mathrm{CH}), 70.98(\mathrm{CH}), 70.98\left(\mathrm{CH}_{2}\right), 71.23\left(\mathrm{CH}_{2}\right), 71.47\left(\mathrm{CH}_{2}\right), 71.76\left(\mathrm{CH}_{2}\right), 71.94\left(\mathrm{CH}_{2}\right), 77.62(\mathrm{CH})$, $79.18(\mathrm{CH}), 79.20(\mathrm{CH}), 79.21(\mathrm{CH}), 80.07(\mathrm{CH}), 80.16(\mathrm{CH}), 80.20(\mathrm{CH}), 80.78(\mathrm{CH}), 81.18$ $(\mathrm{CH}), 81.20(\mathrm{CH}), 81.46(\mathrm{CH}), 81.51(\mathrm{CH}), 81.57(\mathrm{CH}), 81.70(\mathrm{CH}), 81.81(2 \times \mathrm{CH}), 82.06(2 \times$ CH), $82.21(\mathrm{CH}), 82.30(\mathrm{CH}), 82.96(\mathrm{CH}), 96.97(\mathrm{CH}), 97.98(\mathrm{CH}), 98.24(\mathrm{CH}), 99.02(\mathrm{CH}), 99.06$ (CH), $99.15(\mathrm{CH}), 99.39(\mathrm{CH}), 101.04(\mathrm{C})$; MS (ESI $-\mathrm{TOF}): m / z(\%) 1435$ [(M + Na) $\left.{ }^{+}, 100\right]$; HRMS (ESI'-TOF): $m / z[\mathrm{M}+\mathrm{Na}]^{+}$calcd for $\mathrm{C}_{62} \mathrm{H}_{108} \mathrm{NaO}_{35}$ 1435.6569; found 1435.6605. Anal. calcd for $\mathrm{C}_{62} \mathrm{H}_{108} \mathrm{O}_{35}:$ C, 52.68; H, 7.70. Found: C, 52.31; H, 7.57. 
Method B: A solution of alcohol $1(100 \mathrm{mg}, 0.071 \mathrm{mmol})$ in dry $\mathrm{CH}_{2} \mathrm{Cl}_{2}$ (2.9 mL) containing $\mathrm{DIB}(50.3 \mathrm{mg}, 0.156 \mathrm{mmol})$ and $\mathrm{I}_{2}(18 \mathrm{mg}, 0.071 \mathrm{mmol})$ was stirred under nitrogen at $30{ }^{\circ} \mathrm{C}$ for $0.5 \mathrm{~h}$ while irradiated with two $80 \mathrm{~W}$ tungsten-filament lamps. The reaction mixture was then poured into $10 \%$ aqueous $\mathrm{Na}_{2} \mathrm{~S}_{2} \mathrm{O}_{3}$, extracted with $\mathrm{CH}_{2} \mathrm{Cl}_{2}$, dried over $\mathrm{Na}_{2} \mathrm{SO}_{4}$, and concentrated. The residue was purified by silica gel [Merck $60 \mathrm{PF}(0.063-0.2 \mathrm{~mm})$ ] column chromatography (hexanes-acetone, 65:35) to give $2(36.3 \mathrm{mg}, 0.026 \mathrm{mmol}, 36 \%)$ and cyclo-(5R)-5 $5^{\mathrm{VII}}-\mathrm{O}$-acetyl2,3,6-tri-O-methyl- $\alpha$-D-xylo-hexos-5-ulopyranosyl-(1 $\rightarrow 4)-2,3-d i-O$-methyl- $\alpha$-D-glucopyranosyl$[(1 \rightarrow 4)-2,3,6 \text {-tri- } O \text {-methyl- } \alpha \text {-D-glucopyranosyl }]_{5}$ (3) (62.9 mg, $\left.0.043 \mathrm{mmol}, 61 \%\right)$. Compound 3: colorless oil, $[\alpha]_{\mathrm{D}}=+134.5\left(c\right.$ 1.32, $\left.\mathrm{CHCl}_{3}\right)$; IR (film): 3524, 2929, 1734, 1109, $1041 \mathrm{~cm}^{-1} ;{ }^{1} \mathrm{H}$ $\operatorname{NMR}\left(500 \mathrm{MHz}, \mathrm{CDCl}_{3}\right): \delta 2.06(\mathrm{~s}, 3 \mathrm{H}), 3.14(\mathrm{dd}, J=10.1,3.5 \mathrm{~Hz}, 1 \mathrm{H}), 3.15(\mathrm{dd}, J=10.1,3.8$ $\mathrm{Hz}, 1 \mathrm{H}), 3.18(\mathrm{dd}, J=9.5,3.2 \mathrm{~Hz}, 1 \mathrm{H}), 3.19(\mathrm{dd}, J=10.1,3.5 \mathrm{~Hz}, 1 \mathrm{H}), 3.20(\mathrm{dd}, J=10.1,3.5 \mathrm{~Hz}$, 1H), $3.22(\mathrm{dd}, J=10.1,3.5 \mathrm{~Hz}, 1 \mathrm{H}), 3.31(\mathrm{dd}, J=9.8,9.8 \mathrm{~Hz}, 1 \mathrm{H}), 3.367(\mathrm{~s}, 6 \mathrm{H}), 3.373(\mathrm{~s}, 3 \mathrm{H})$, $3.38(\mathrm{~s}, 3 \mathrm{H}), 3.39$ (s, 3H), $3.40(\mathrm{~s}, 3 \mathrm{H}), 3.42(\mathrm{~s}, 3 \mathrm{H}), 3.45$ (s, 3H), $3.475(\mathrm{~s}, 3 \mathrm{H}), 3.478(\mathrm{~s}, 3 \mathrm{H}), 3.49$ $(\mathrm{s}, 3 \mathrm{H}), 3.51(\mathrm{~s}, 3 \mathrm{H}), 3.53(\mathrm{~s}, 3 \mathrm{H}), 3.58(\mathrm{~s}, 3 \mathrm{H}), 3.61(\mathrm{~s}, 3 \mathrm{H}), 3.62(\mathrm{~s}, 6 \mathrm{H}), 3.637(\mathrm{~s}, 6 \mathrm{H}), 3.644(\mathrm{~s}$, 3H), $3.96\left(\mathrm{~d}, J=10.1 \mathrm{~Hz}, 1 \mathrm{H}, \mathrm{H}^{\mathrm{VII}}\right), 3.99\left(\mathrm{~d}, J=9.8 \mathrm{~Hz}, 1 \mathrm{H}, \mathrm{H}^{\mathrm{V}} \mathrm{b}^{\mathrm{VII}}\right), 4.04$ (br d, $J=1.9 \mathrm{~Hz}, 1 \mathrm{H}$, $\left.\mathrm{H} 4^{\mathrm{VII}}\right), 4.20\left(\mathrm{dd}, J=2.5,2.5 \mathrm{~Hz}, 1 \mathrm{H}, \mathrm{H} 3^{\mathrm{VII}}\right), 4.31(\mathrm{br} \mathrm{d}, J=11.7 \mathrm{~Hz}, 1 \mathrm{H}), 5.01(\mathrm{~d}, J=3.2 \mathrm{~Hz}, 1 \mathrm{H})$, $5.04(\mathrm{~d}, J=3.2 \mathrm{~Hz}, 1 \mathrm{H}), 5.060(\mathrm{~d}, J=2.8 \mathrm{~Hz}, 1 \mathrm{H}), 5.061(\mathrm{~d}, J=4.1 \mathrm{~Hz}, 1 \mathrm{H}), 5.12(\mathrm{~d}, J=3.8 \mathrm{~Hz}$, 1H), $5.14\left(\mathrm{~d}, J=1.9 \mathrm{~Hz}, 1 \mathrm{H}, \mathrm{H} 1^{\mathrm{VII}}\right), 5.18(\mathrm{~d}, J=3.5 \mathrm{~Hz}, 1 \mathrm{H}) ;{ }^{1} \mathrm{H}$ NMR $\left(500 \mathrm{MHz}, \mathrm{CDCl}_{3}, 1 \mathrm{D}-\right.$ TOCSY, irradiation at $\left.\mathrm{H} 3^{\mathrm{VII}}, 4.20 \mathrm{ppm}\right): \delta 3.41$ (br s, $\left.1 \mathrm{H}, \mathrm{H} 2^{\mathrm{VII}}\right), 4.04\left(\right.$ br d, $J=1.9 \mathrm{~Hz}, 1 \mathrm{H}, \mathrm{H} 4^{\mathrm{VII}}$ ), $5.14\left(\mathrm{~d}, J=1.9 \mathrm{~Hz}, 1 \mathrm{H}, \mathrm{H} 1^{\mathrm{VII}}\right) ;{ }^{1} \mathrm{H}$ NMR $\left(500 \mathrm{MHz}, \mathrm{C}_{6} \mathrm{D}_{6}\right): \delta 1.72(\mathrm{~s}, 3 \mathrm{H}), 3.10(\mathrm{dd}, J=9.1,3.2$ $\mathrm{Hz}, 1 \mathrm{H}), 3.13(\mathrm{dd}, J=9.8,3.5 \mathrm{~Hz}, 1 \mathrm{H}), 3.19(\mathrm{~s}, 3 \mathrm{H}), 3.20(\mathrm{~s}, 3 \mathrm{H}), 3.26(\mathrm{~s}, 3 \mathrm{H}), 3.27$ (s, 3H), 3.28 (s, 3H), $3.30(\mathrm{~s}, 6 \mathrm{H}), 3.31(\mathrm{~s}, 3 \mathrm{H}), 3.32(\mathrm{~s}, 3 \mathrm{H}), 3.39(\mathrm{~s}, 3 \mathrm{H}), 3.40(\mathrm{~s}, 6 \mathrm{H}), 3.41(\mathrm{~s}, 3 \mathrm{H}), 3.46$ (s, $3 \mathrm{H}), 3.60(\mathrm{~s}, 3 \mathrm{H}), 3.68(\mathrm{~s}, 3 \mathrm{H}), 3.74(\mathrm{~s}, 3 \mathrm{H}), 3.75(\mathrm{~s}, 3 \mathrm{H}), 3.76(\mathrm{~s}, 3 \mathrm{H}), 3.80(\mathrm{~s}, 3 \mathrm{H}), 5.07(\mathrm{~d}, J=$ 
$3.2 \mathrm{~Hz}, 1 \mathrm{H}), 5.10(\mathrm{~d}, J=2.8 \mathrm{~Hz}, 1 \mathrm{H}), 5.20(\mathrm{~d}, J=3.2 \mathrm{~Hz}, 1 \mathrm{H}), 5.27(\mathrm{~d}, J=3.8 \mathrm{~Hz}, 1 \mathrm{H}), 5.32(\mathrm{~d}, J$ $=3.5 \mathrm{~Hz}, 1 \mathrm{H}), 5.45(\mathrm{~d}, J=3.8 \mathrm{~Hz}, 1 \mathrm{H}), 5.52\left(\mathrm{~d}, J=1.6 \mathrm{~Hz}, 1 \mathrm{H}, \mathrm{H} 1{ }^{\mathrm{II}}\right) ;{ }^{13} \mathrm{C}$ NMR $(125.7 \mathrm{MHz}$, $\left.\mathrm{CDCl}_{3}\right): \delta 22.08\left(\mathrm{CH}_{3}\right), 57.27\left(\mathrm{CH}_{3}\right), 57.45\left(\mathrm{CH}_{3}\right), 58.04\left(\mathrm{CH}_{3}\right), 58.37\left(\mathrm{CH}_{3}\right), 58.65\left(\mathrm{CH}_{3}\right), 58.68$ $\left(\mathrm{CH}_{3}\right), 58.72\left(\mathrm{CH}_{3}\right), 58.86\left(2 \times \mathrm{CH}_{3}\right), 58.93\left(\mathrm{CH}_{3}\right), 58.96\left(3 \times \mathrm{CH}_{3}\right), 59.17\left(\mathrm{CH}_{3}\right), 60.16\left(\mathrm{CH}_{2}\right)$, $61.18\left(\mathrm{CH}_{3}\right), 61.25\left(\mathrm{CH}_{3}\right), 61.34\left(\mathrm{CH}_{3}\right), 61.45\left(\mathrm{CH}_{3}\right), 61.52\left(\mathrm{CH}_{3}\right), 61.67\left(\mathrm{CH}_{3}\right), 70.33\left(\mathrm{CH}_{2}\right.$, $\left.\mathrm{C6}^{\mathrm{VII}}\right), 70.60(\mathrm{CH}), 70.80\left(\mathrm{CH}_{2}\right), 70.91(\mathrm{CH}), 70.91\left(\mathrm{CH}_{2}\right), 71.01\left(\mathrm{CH}, \mathrm{C} 4{ }^{\mathrm{VII}}\right), 71.13(2 \times \mathrm{CH})$, $71.13\left(2 \times \mathrm{CH}_{2}\right), 71.31\left(\mathrm{CH}_{2}\right), 71.35(\mathrm{CH}), 71.47(\mathrm{CH}), 75.22\left(\mathrm{CH}, \mathrm{C}^{\mathrm{VII}}\right), 76.64\left(\mathrm{CH}, \mathrm{C} 2^{\mathrm{VII}}\right), 79.43$ $(\mathrm{CH}), 79.93(\mathrm{CH}), 80.61(\mathrm{CH}), 81.20(\mathrm{CH}), 81.31(\mathrm{CH}), 81.47(\mathrm{CH}), 81.59(\mathrm{CH}), 81.74(2 \times \mathrm{CH})$, $81.75(\mathrm{CH}), 81.97(\mathrm{CH}), 82.00(\mathrm{CH}), 82.08(\mathrm{CH}), 82.22(\mathrm{CH}), 82.26(\mathrm{CH}), 82.28(\mathrm{CH}), 82.30$ $(\mathrm{CH}), 83.15(\mathrm{CH}), 97.59\left(\mathrm{CH}, \mathrm{C} 1^{\mathrm{VII}}\right), 99.25(3 \times \mathrm{CH}), 99.52(\mathrm{CH}), 99.87(\mathrm{CH}), 100.34(\mathrm{CH})$, $105.03\left(\mathrm{C}, \mathrm{C} 5^{\mathrm{VII}}\right), 170.19$ (C); MS (ESI $\left.{ }^{+}-\mathrm{TOF}\right): \mathrm{m} / z(\%) 1495\left[(\mathrm{M}+\mathrm{Na})^{+}, 100\right]$; HRMS $\left(\mathrm{ESI}^{+}-\right.$ TOF): $m / z[\mathrm{M}+\mathrm{Na}]^{+}$calcd for $\mathrm{C}_{64} \mathrm{H}_{112} \mathrm{NaO}_{37}$ 1495.6780; found 1495.6727. Anal. calcd for $\mathrm{C}_{64} \mathrm{H}_{112} \mathrm{O}_{37}: \mathrm{C}, 52.17 ; \mathrm{H}, 7.66$. Found: C, 52.29; H, 7.58.

Oxidative HAT of $2^{I-V I I}, 3^{I-V I I}, 6^{I I-V I I}$-icosa-O-methyl- $\beta$-cyclomaltoheptaose (1) with bis(4-tertbutylbenzoyloxy)iodobenzene and iodine. A solution of alcohol 1 (30 mg, $0.021 \mathrm{mmol})$ in dry $\mathrm{CH}_{2} \mathrm{Cl}_{2}(0.85 \mathrm{~mL})$ containing bis(4-tert-butylbenzoyloxy)iodobenzene $(25.8 \mathrm{mg}, 0.046 \mathrm{mmol})$ and $\mathrm{I}_{2}(5.3 \mathrm{mg}, 0.021 \mathrm{mmol})$ was stirred under nitrogen at $25^{\circ} \mathrm{C}$ for $1.5 \mathrm{~h}$ while irradiated with two 80 W tungsten-filament lamps. The reaction mixture was then directly loaded onto a silica gel (TLC Silica gel $60 \mathrm{~F}_{254}$, scraped from Merck Aluminum sheets) column chromatography (hexanesacetone, $70: 30 \rightarrow 65: 35)$ to give $2(13.7 \mathrm{mg}, 0.01 \mathrm{mmol}, 46 \%)$.

Oxidative HAT of $2^{I-V I I}, 3^{I-V I I}, 6^{I I-V I I}$-icosa-O-methyl- $\beta$-cyclomaltoheptaose (1) with bis $(2,4,6$ trimethylbenzoyloxy)iodobenzene and iodine. A solution of alcohol 1 (90 mg, $0.064 \mathrm{mmol})$ in dry $\mathrm{CH}_{2} \mathrm{Cl}_{2}$ (2.6 mL) containing bis(2,4,6-trimethylbenzoyloxy)iodobenzene (101 mg, $\left.0.191 \mathrm{mmol}\right)$ 
and $\mathrm{I}_{2}(16.2 \mathrm{mg}, 0.064 \mathrm{mmol})$ was stirred under nitrogen at $28{ }^{\circ} \mathrm{C}$ for $2 \mathrm{~h}$ while irradiated with two $80 \mathrm{~W}$ tungsten-filament lamps. The reaction mixture was then directly loaded onto a silica gel (TLC Silica gel $60 \mathrm{~F}_{254}$, scraped from Merck Aluminum sheets) column chromatography (hexanesacetone, 70:30) to give $2(19.3 \mathrm{mg}, 0.014 \mathrm{mmol}, 21 \%)$ and cyclo- $(5 R)-5^{\mathrm{VII}}-O-(2,4,6-$ trimethylbenzoyl)-2,3,6-tri-O-methyl- $\alpha$-D- $x y l o$-hexos-5-ulopyranosyl-(1 $\rightarrow 4)-2,3-$ di- $O$-methyl- $\alpha$ -

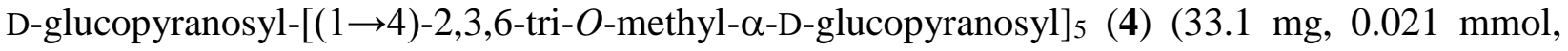
33\%). Compound 4: amorphous solid, $[\alpha]_{\mathrm{D}}+132.5$ (c 1.10, $\mathrm{CHCl}_{3}$ ); IR (film): 2928, 1727, 1455, 1368, 1192, 1107, $1043 \mathrm{~cm}^{-1} ;{ }^{1} \mathrm{H}$ NMR (500 MHz, $\left.\mathrm{CDCl}_{3}\right): \delta 2.26$ (s, 3H), $2.36(\mathrm{~s}, 6 \mathrm{H}), 3.14$ (dd, $J=6.9,3.2 \mathrm{~Hz}, 1 \mathrm{H}), 3.17(\mathrm{~m}, 5 \mathrm{H}), 3.21(\mathrm{dd}, J=5.7,3.8 \mathrm{~Hz}, 1 \mathrm{H}), 3.32(\mathrm{~s}, 3 \mathrm{H}), 3.37(\mathrm{~s}, 3 \mathrm{H}), 3.38$ (s, 3H), $3.39(\mathrm{~s}, 3 \mathrm{H}), 3.40(\mathrm{~s}, 3 \mathrm{H}), 3.40(\mathrm{~s}, 3 \mathrm{H}), 3.41(\mathrm{~s}, 3 \mathrm{H}), 3.44(\mathrm{~s}, 3 \mathrm{H}), 3.48(\mathrm{~s}, 3 \mathrm{H}), 3.48(\mathrm{~s}$, 3H), $3.49(\mathrm{~s}, 3 \mathrm{H}), 3.51(\mathrm{~s}, 6 \mathrm{H}), 3.58(\mathrm{~s}, 3 \mathrm{H}), 3.62(\mathrm{~s}, 6 \mathrm{H}), 3.64(\mathrm{~s}, 3 \mathrm{H}), 3.65(\mathrm{~s}, 3 \mathrm{H}), 3.66(\mathrm{~s}, 3 \mathrm{H})$, 4.36 (br d, $J=12.0 \mathrm{~Hz}), 5.04(\mathrm{~d}, J=2.8 \mathrm{~Hz}, 1 \mathrm{H}), 5.07(\mathrm{~m}, 3 \mathrm{H}), 5.13(\mathrm{~d}, J=3.8 \mathrm{~Hz}, 1 \mathrm{H}), 5.18$ (d, $J=3.8 \mathrm{~Hz}, 1 \mathrm{H}), 5.36(\mathrm{~d}, J=1.9 \mathrm{~Hz}, 1 \mathrm{H}), 6.81(\mathrm{~s}, 2 \mathrm{H}) ;{ }^{1} \mathrm{H} \mathrm{NMR}\left(500 \mathrm{MHz}, \mathrm{C}_{6} \mathrm{D}_{6}\right): \delta 2.03(\mathrm{~s}, 3 \mathrm{H})$, $2.57(\mathrm{~s}, 6 \mathrm{H}), 3.12(\mathrm{~s}, 3 \mathrm{H}), 3.14(\mathrm{~s}, 3 \mathrm{H}), 3.20(\mathrm{~s}, 3 \mathrm{H}), 3.28(\mathrm{~s}, 3 \mathrm{H}), 3.29(\mathrm{~s}, 3 \mathrm{H}), 3.29(\mathrm{~s}, 3 \mathrm{H}), 3.30$ (s, 3H), $3.31(\mathrm{~s}, 3 \mathrm{H}), 3.32(\mathrm{~s}, 3 \mathrm{H}), 3.40(\mathrm{~s}, 3 \mathrm{H}), 3.41(\mathrm{~s}, 3 \mathrm{H}), 3.42(\mathrm{~s}, 3 \mathrm{H}), 3.42(\mathrm{~s}, 3 \mathrm{H}), 3.44(\mathrm{~s}$, $3 \mathrm{H}), 3.60(\mathrm{~s}, 3 \mathrm{H}), 3.68(\mathrm{~s}, 3 \mathrm{H}), 3.73(\mathrm{~s}, 3 \mathrm{H}), 3.75(\mathrm{~s}, 3 \mathrm{H}), 3.77(\mathrm{~s}, 3 \mathrm{H}), 3.79(\mathrm{~s}, 3 \mathrm{H}), 4.67(\mathrm{~d}, J=$ $9.5 \mathrm{~Hz}, 1 \mathrm{H}), 5.09$ (d, $J=3.2 \mathrm{~Hz}, 1 \mathrm{H}), 5.17(\mathrm{~d}, J=2.8 \mathrm{~Hz}, 1 \mathrm{H}), 5.21(\mathrm{~d}, J=3.5 \mathrm{~Hz}, 1 \mathrm{H}), 5.26(\mathrm{~d}, J$ $=3.8 \mathrm{~Hz}, 1 \mathrm{H}), 5.33(\mathrm{~d}, J=3.5 \mathrm{~Hz}, 1 \mathrm{H}), 5.47(\mathrm{~d}, J=3.8 \mathrm{~Hz}, 1 \mathrm{H}), 5.74(\mathrm{~d}, J=1.9 \mathrm{~Hz}, 1 \mathrm{H}), 6.67(\mathrm{~s}$, $2 \mathrm{H}) ;{ }^{13} \mathrm{C} \mathrm{NMR}\left(125.7 \mathrm{MHz}, \mathrm{CDCl}_{3}\right): \delta 19.53\left(2 \times \mathrm{CH}_{3}\right), 21.08\left(\mathrm{CH}_{3}\right), 57.12\left(\mathrm{CH}_{3}\right), 57.27\left(\mathrm{CH}_{3}\right)$, $58.07\left(\mathrm{CH}_{3}\right), 58.37\left(\mathrm{CH}_{3}\right), 58.44\left(\mathrm{CH}_{3}\right), 58.68\left(\mathrm{CH}_{3}\right), 58.70\left(\mathrm{CH}_{3}\right), 58.74\left(\mathrm{CH}_{3}\right), 58.88\left(2 \times \mathrm{CH}_{3}\right)$, $58.95\left(\mathrm{CH}_{3}\right), 58.98\left(\mathrm{CH}_{3}\right), 58.99\left(\mathrm{CH}_{3}\right), 59.19\left(\mathrm{CH}_{3}\right), 60.51\left(\mathrm{CH}_{2}\right), 61.00\left(\mathrm{CH}_{3}\right), 61.28\left(\mathrm{CH}_{3}\right), 61.36$ $\left(\mathrm{CH}_{3}\right), 61.50\left(\mathrm{CH}_{3}\right), 61.56\left(\mathrm{CH}_{3}\right), 61.72\left(\mathrm{CH}_{3}\right), 69.93\left(\mathrm{CH}_{2}\right), 70.62(\mathrm{CH}), 70.87\left(\mathrm{CH}_{2}\right), 70.91\left(\mathrm{CH}_{2}\right)$, $70.91(\mathrm{CH}), 71.04(\mathrm{CH}), 71.11(\mathrm{CH}), 71.16\left(\mathrm{CH}_{2}\right), 71.20\left(\mathrm{CH}_{2}\right), 71.40(\mathrm{CH}), 71.40\left(\mathrm{CH}_{2}\right), 71.51$ 
$(\mathrm{CH}), 71.83(\mathrm{CH}), 75.16(\mathrm{CH}), 76.44(\mathrm{CH}), 78.98(\mathrm{CH}), 80.10(\mathrm{CH}), 80.63(\mathrm{CH}), 81.31(\mathrm{CH})$, $81.39(\mathrm{CH}), 81.52(\mathrm{CH}), 81.63(\mathrm{CH}), 81.78(2 \times \mathrm{CH}), 81.84(\mathrm{CH}), 81.94(\mathrm{CH}), 81.954(\mathrm{CH}), 82.13$ $(\mathrm{CH}), 82.26(\mathrm{CH}), 82.31(\mathrm{CH}), 82.36(2 \times \mathrm{CH}), 83.19(\mathrm{CH}), 97.40(\mathrm{CH}), 99.13(\mathrm{CH}), 99.26(\mathrm{CH})$, $99.40(\mathrm{CH}), 99.57(\mathrm{CH}), 99.88(\mathrm{CH}), 100.26(\mathrm{CH}), 106.38(\mathrm{C}), 128.37(2 \times \mathrm{CH}), 131.24(\mathrm{C})$, $135.26(2 \times \mathrm{C}), 139.10(\mathrm{C}), 169.51(\mathrm{C}) ; \mathrm{MS}\left(\mathrm{ESI}^{+}-\mathrm{TOF}\right): \mathrm{m} / z(\%) 1599\left[(\mathrm{M}+\mathrm{Na})^{+}, 100\right] ; \mathrm{HRMS}$ (ESI ${ }^{+}$-TOF): $m / z[\mathrm{M}+\mathrm{Na}]^{+}$calcd for $\mathrm{C}_{72} \mathrm{H}_{120} \mathrm{NaO}_{37}$ 1599.7406; found: 1599.7413 . Anal. calcd for $\mathrm{C}_{72} \mathrm{H}_{120} \mathrm{O}_{37}: \mathrm{C}, 54.81 ; \mathrm{H}, 7.67$. Found: $\mathrm{C}, 54.60 ; \mathrm{H}, 7.66$.

Oxidative HAT of $2^{I-V I}, 3^{I-V I}, 6^{I I-V I}$-heptadeca-O-methyl- $\alpha$-cyclomaltohexaose (5). Method A: A solution of alcohol $5^{25}(30 \mathrm{mg}, 0.025 \mathrm{mmol})$ in dry $\mathrm{CH}_{2} \mathrm{Cl}_{2}(1 \mathrm{~mL})$ containing DIB (17.6 mg, 0.055 mmol) and $\mathrm{I}_{2}(6.3 \mathrm{mg}, 0.025 \mathrm{mmol})$ was stirred under nitrogen at $21{ }^{\circ} \mathrm{C}$ for $1 \mathrm{~h}$ while irradiated with two $80 \mathrm{~W}$ tungsten-filament lamps. An excess of solid $\mathrm{Na}_{2} \mathrm{~S}_{2} \mathrm{O}_{3}$ was then added and stirring continued until complete disappearance of the iodine color. The reaction mixture was then filtered and concentrated under reduced pressure. Silica gel [Merck 60 PF $(0.063-0.2 \mathrm{~mm})]$ column chromatography of the reaction residue (hexanes-acetone, 65:35) afforded cyclo- $5^{\mathrm{VI}}, 6^{\mathrm{I}}$-anhydro$\left(5^{\mathrm{VI}} R\right)$-(2,3,6-tri- $O$-methyl- $\alpha$-D-xylo-hexos-5-ulopyranosyl)-( $\left.1 \rightarrow 4\right)$-2,3-di- $O$-methyl- $\alpha$-D-

glucopyranosyl-[(1 $\rightarrow 4)$-2,3,6-tri- $O$-methyl- $\alpha$-D-glucopyranosyl $]_{4}(6)(17.7 \mathrm{mg}, 0.015 \mathrm{mmol}, 59 \%)$ as a colorless oil: $[\alpha]_{\mathrm{D}}+127.6\left(c\right.$ 1.24, $\left.\mathrm{CHCl}_{3}\right)$; IR (film): 3052, 2929, $28321454,1365,1196,1107$ $\mathrm{cm}^{-1}$; ${ }^{1} \mathrm{H}$ NMR (500 MHz, $\left.\mathrm{CDCl}_{3}\right): \delta 3.12(\mathrm{dd}, J=10.1,3.2 \mathrm{~Hz}, 1 \mathrm{H}), 3.14(\mathrm{dd}, J=9.9,3.3 \mathrm{~Hz}$, $1 \mathrm{H}), 3.15(\mathrm{dd}, J=9.3,3.6 \mathrm{~Hz}, 1 \mathrm{H}), 3.18(\mathrm{dd}, J=9.8,3.8 \mathrm{~Hz}, 1 \mathrm{H}), 3.19(\mathrm{dd}, J=9.8,3.5 \mathrm{~Hz}, 1 \mathrm{H})$, $3.25(\mathrm{dd}, J=9.8,3.2 \mathrm{~Hz}, 1 \mathrm{H}), 3.357$ (s, 3H), 3.360 (s, 3H), 3.37 (s, 3H), 3.378 (s, 3H), 3.379 (s, 3H), 3.469 (s, 3H), 3.470 (s, 3H), 3.483 (s, 3H), $3.488(\mathrm{~s}, 3 \mathrm{H}), 3.493$ (s, 3H), 3.51 (s, 3H), $3.58(\mathrm{~s}$, 3H), $3.60(\mathrm{~s}, 3 \mathrm{H}), 3.627$ (s, 3H), $3.632(\mathrm{~s}, 3 \mathrm{H}), 3.70(\mathrm{~s}, 3 \mathrm{H}), 3.73(\mathrm{~s}, 3 \mathrm{H}), 4.98(\mathrm{~d}, J=3.8 \mathrm{~Hz}, 1 \mathrm{H})$, $5.00(\mathrm{~d}, J=3.5 \mathrm{~Hz}, 1 \mathrm{H}), 5.03(\mathrm{~d}, J=3.8 \mathrm{~Hz}, 1 \mathrm{H}), 5.04(\mathrm{~d}, J=3.5 \mathrm{~Hz}, 1 \mathrm{H}), 5.06(\mathrm{~d}, J=3.2 \mathrm{~Hz}$, 
$1 \mathrm{H}), 5.19(\mathrm{~d}, J=3.5 \mathrm{~Hz}, 1 \mathrm{H}) ;{ }^{13} \mathrm{C} \mathrm{NMR}\left(125.7 \mathrm{MHz}, \mathrm{CDCl}_{3}\right): \delta 57.65\left(\mathrm{CH}_{3}\right), 57.80\left(2 \times \mathrm{CH}_{3}\right)$, $57.96\left(\mathrm{CH}_{3}\right), 58.31\left(\mathrm{CH}_{3}\right), 58.34\left(\mathrm{CH}_{3}\right), 58.67\left(\mathrm{CH}_{3}\right), 58.72\left(\mathrm{CH}_{3}\right), 58.96\left(\mathrm{CH}_{3}\right), 59.00\left(\mathrm{CH}_{3}\right), 59.07$ $\left(\mathrm{CH}_{3}\right), 61.26\left(\mathrm{CH}_{3}\right), 61.45\left(\mathrm{CH}_{3}\right), 61.51\left(\mathrm{CH}_{3}\right), 61.89\left(\mathrm{CH}_{3}\right), 61.98\left(\mathrm{CH}_{3}\right), 62.22\left(\mathrm{CH}_{3}\right), 63.83$ $\left(\mathrm{CH}_{2}\right), 66.20(\mathrm{CH}), 70.87(\mathrm{CH}), 70.92(2 \times \mathrm{CH}), 71.03\left(\mathrm{CH}_{2}\right), 71.22\left(\mathrm{CH}_{2}\right), 71.55\left(\mathrm{CH}_{2}\right), 71.55$ $(\mathrm{CH}), 71.88\left(\mathrm{CH}_{2}\right), 71.97\left(\mathrm{CH}_{2}\right), 78.28(\mathrm{CH}), 80.19(\mathrm{CH}), 80.59(\mathrm{CH}), 80.72(\mathrm{CH}), 80.84(\mathrm{CH})$, $81.04(\mathrm{CH}), 81.06(\mathrm{CH}), 81.25(\mathrm{CH}), 81.41(\mathrm{CH}), 81.51(\mathrm{CH}), 81.72(\mathrm{CH}), 82.00(\mathrm{CH}), 82.07$ $(\mathrm{CH}), 82.10(\mathrm{CH}), 82.29(\mathrm{CH}), 82.31(\mathrm{CH}), 82.59(\mathrm{CH}), 82.67(\mathrm{CH}), 97.35(\mathrm{CH}), 97.59(\mathrm{CH})$, $98.85(\mathrm{CH}), 99.55(\mathrm{CH}), 99.94(\mathrm{CH}), 100.08(\mathrm{CH}), 100.79(\mathrm{C}) ;{ }^{1} \mathrm{H}$ NMR (500 MHz, $\left.\mathrm{C}_{6} \mathrm{D}_{6}\right): \delta 3.15$ $(\mathrm{dd}, J=9.8,3.5 \mathrm{~Hz}, 1 \mathrm{H}), 3.16(\mathrm{dd}, J=9.1,4.4 \mathrm{~Hz}, 1 \mathrm{H}), 3.19(\mathrm{dd}, J=9.8,3.8 \mathrm{~Hz}, 1 \mathrm{H}), 3.20(\mathrm{dd}, J$ $=9.9,3.3 \mathrm{~Hz}, 1 \mathrm{H}), 3.24(\mathrm{dd}, J=9.8,3.2 \mathrm{~Hz}, 1 \mathrm{H}), 3.25(\mathrm{~s}, 3 \mathrm{H}), 3.268(\mathrm{~s}, 3 \mathrm{H}), 3.273(\mathrm{~s}, 3 \mathrm{H}), 3.28$ (s, 3H), $3.30(\mathrm{~s}, 3 \mathrm{H}), 3.31(\mathrm{~s}, 3 \mathrm{H}), 3.317$ (s, 3H), $3.320(\mathrm{~s}, 3 \mathrm{H}), 3.34$ (s, 3H), $3.46(\mathrm{~s}, 3 \mathrm{H}), 3.50(\mathrm{~s}$, 3H), $3.55(\mathrm{dd}, J=8.8,7.9 \mathrm{~Hz}, 1 \mathrm{H}), 3.67(\mathrm{~s}, 3 \mathrm{H}), 3.68(\mathrm{~s}, 3 \mathrm{H}), 3.70(\mathrm{~s}, 3 \mathrm{H}), 3.80(\mathrm{~s}, 3 \mathrm{H}), 3.82(\mathrm{~s}$, 3H), $3.83(\mathrm{~s}, 3 \mathrm{H}), 4.16(\mathrm{dd}, J=9.5,9.5 \mathrm{~Hz}, 1 \mathrm{H}), 4.27(\mathrm{~d}, J=9.1 \mathrm{~Hz}, 1 \mathrm{H}), 4.37(\mathrm{~d}, J=10.1 \mathrm{~Hz}$, 1H), 4.55 (ddd, $J=10.1,5.0,1.3 \mathrm{~Hz}, 1 \mathrm{H}), 5.02(\mathrm{~d}, J=3.8 \mathrm{~Hz}, 1 \mathrm{H}), 5.17(\mathrm{~d}, J=3.3 \mathrm{~Hz}, 1 \mathrm{H}), 5.18$ $(\mathrm{d}, J=3.5 \mathrm{~Hz}, 1 \mathrm{H}), 5.19(\mathrm{~d}, J=3.2 \mathrm{~Hz}, 1 \mathrm{H}), 5.22(\mathrm{~d}, J=3.5 \mathrm{~Hz}, 1 \mathrm{H}), 5.24(\mathrm{~d}, J=3.5 \mathrm{~Hz}, 1 \mathrm{H}) ;{ }^{13} \mathrm{C}$ NMR (125.7 MHz, $\left.\mathrm{C}_{6} \mathrm{D}_{6}\right): \delta 57.22\left(\mathrm{CH}_{3}\right), 57.70\left(\mathrm{CH}_{3}\right), 57.84\left(\mathrm{CH}_{3}\right), 57.93\left(\mathrm{CH}_{3}\right), 58.15\left(\mathrm{CH}_{3}\right)$, $58.17\left(\mathrm{CH}_{3}\right), 58.61\left(\mathrm{CH}_{3}\right), 58.72\left(\mathrm{CH}_{3}\right), 58.91\left(\mathrm{CH}_{3}\right), 59.00\left(\mathrm{CH}_{3}\right), 59.03\left(\mathrm{CH}_{3}\right), 61.31\left(\mathrm{CH}_{3}\right), 61.53$ $\left(\mathrm{CH}_{3}\right), 61.58\left(\mathrm{CH}_{3}\right), 61.79\left(\mathrm{CH}_{3}\right), 62.08\left(\mathrm{CH}_{3}\right), 62.15\left(\mathrm{CH}_{3}\right), 64.19\left(\mathrm{CH}_{2}\right), 66.85(\mathrm{CH}), 71.42(\mathrm{CH})$, $71.64(\mathrm{CH}), 71.67\left(\mathrm{CH}_{2}\right), 71.83(\mathrm{CH}), 72.52(\mathrm{CH}), 72.87\left(\mathrm{CH}_{2}\right), 72.92\left(2 \times \mathrm{CH}_{2}\right), 73.10\left(\mathrm{CH}_{2}\right)$, $79.14(\mathrm{CH}), 81.49(\mathrm{CH}), 81.60(2 \times \mathrm{CH}), 81.69(\mathrm{CH}), 81.76(\mathrm{CH}), 81.89(\mathrm{CH}), 82.03(\mathrm{CH}), 82.09$ $(\mathrm{CH}), 82.35(\mathrm{CH}), 82.67(\mathrm{CH}), 82.92(\mathrm{CH}), 82.94(2 \times \mathrm{CH}), 82.97(\mathrm{CH}), 83.34(\mathrm{CH}), 83.37(\mathrm{CH})$, $83.48(\mathrm{CH}), 98.07(\mathrm{CH}), 98.58(\mathrm{CH}), 99.11(\mathrm{CH}), 99.17(\mathrm{CH}), 99.95(\mathrm{CH}), 100.42(\mathrm{CH}), 101.31$ (C); MS (ESI $\left.{ }^{+}-\mathrm{TOF}\right): m / z(\%) 1231\left[(\mathrm{M}+\mathrm{Na})^{+}, 100\right] ; \mathrm{HRMS}\left(\mathrm{ESI}^{+}-\mathrm{TOF}\right): m / z[\mathrm{M}+\mathrm{Na}]^{+}$calcd 
for $\mathrm{C}_{53} \mathrm{H}_{92} \mathrm{NaO}_{30}$ 1231.5571; found 1231.5543. Anal. calcd for $\mathrm{C}_{53} \mathrm{H}_{92} \mathrm{O}_{30}$ : C, 52.64; $\mathrm{H}, 7.67$. Found: C, 52.46; H, 7.76.

Method B: A solution of alcohol 5 (45 mg, $0.037 \mathrm{mmol})$ in dry $\mathrm{CH}_{2} \mathrm{Cl}_{2}(1.5 \mathrm{~mL})$ containing DIB (26.2 $\mathrm{mg}, 0.081 \mathrm{mmol})$ and $\mathrm{I}_{2}(4.7 \mathrm{mg}, 0.019 \mathrm{mmol})$ was stirred under nitrogen at $22{ }^{\circ} \mathrm{C}$ for $1.5 \mathrm{~h}$ while irradiated with two $80 \mathrm{~W}$ tungsten-filament lamps. The reaction mixture was then directly loaded onto a silica gel (TLC Silica gel $60 \mathrm{~F}_{254}$, scraped from Merck Aluminum sheets) column chromatography (hexanes-acetone, $65: 35)$ to give 6 (14 mg, $0.012 \mathrm{mmol}, 31 \%)$ and cyclo-(5R)-5 ${ }^{\mathrm{VI}}-$ $O$-acetyl-2,3,6-tri- $O$-methyl- $\alpha$-D-xylo-hexos-5-ulopyranosyl-(1 $\rightarrow 4)-2,3-$ di- $O$-methyl- $\alpha$-Dglucopyranosyl-[(1 $\rightarrow 4)-2,3,6$-tri- $O$-methyl- $\alpha$-D-glucopyranosyl $]_{4}(7)(13.9 \mathrm{mg}, 0.011 \mathrm{mmol}, 30 \%)$. Compound 7: colorless oil, $[\alpha]_{\mathrm{D}}+127.8$ (c 0.90, $\left.\mathrm{CHCl}_{3}\right)$; IR (film): 3509, 2933, 1758, 1107, 1041 $\mathrm{cm}^{-1} ;{ }^{1} \mathrm{H}$ NMR $\left(500 \mathrm{MHz}, \mathrm{CDCl}_{3}\right): \delta 2.05(\mathrm{~s}, 3 \mathrm{H}), 3.15(\mathrm{dd}, J=10.1,3.2 \mathrm{~Hz}, 1 \mathrm{H}), 3.15(\mathrm{dd}, J=$ 10.1, 3.2 Hz, 1H), $3.16(\mathrm{dd}, J=9.8,3.5 \mathrm{~Hz}, 1 \mathrm{H}), 3.17(\mathrm{dd}, J=10.1,3.5 \mathrm{~Hz}, 1 \mathrm{H}), 3.21$ (dd, $J=9.5$, $3.2 \mathrm{~Hz}, 1 \mathrm{H}), 3.36(\mathrm{~s}, 3 \mathrm{H}), 3.368(\mathrm{~s}, 3 \mathrm{H}), 3.373(\mathrm{~s}, 6 \mathrm{H}), 3.38(\mathrm{~s}, 3 \mathrm{H}), 3.43(\mathrm{~s}, 3 \mathrm{H}), 3.456(\mathrm{~s}, 3 \mathrm{H})$, $3.464(\mathrm{~s}, 3 \mathrm{H}), 3.48(\mathrm{~s}, 3 \mathrm{H}), 3.50(\mathrm{~s}, 3 \mathrm{H}), 3.52(\mathrm{~s}, 3 \mathrm{H}), 3.607(\mathrm{~s}, 3 \mathrm{H}), 3.609(\mathrm{~s}, 3 \mathrm{H}), 3.613(\mathrm{~s}, 3 \mathrm{H})$, $3.62(\mathrm{~s}, 3 \mathrm{H}), 3.64(\mathrm{~s}, 3 \mathrm{H}), 3.68(\mathrm{~s}, 3 \mathrm{H}), 3.91(\mathrm{~d}, J=10.4 \mathrm{~Hz}, 1 \mathrm{H}), 3.98(\mathrm{dd}, J=3.0,3.0 \mathrm{~Hz}, 1 \mathrm{H})$, $4.02(\mathrm{~d}, J=10.4 \mathrm{~Hz}, 1 \mathrm{H}), 4.21(\mathrm{~d}, J=3.2 \mathrm{~Hz}, 1 \mathrm{H}), 4.23(\mathrm{~m}, 1 \mathrm{H}), 4.97(\mathrm{~d}, J=3.5 \mathrm{~Hz}, 1 \mathrm{H}), 5.02(\mathrm{~d}$, $J=3.5 \mathrm{~Hz}, 1 \mathrm{H}), 5.03(\mathrm{~d}, J=3.8 \mathrm{~Hz}, 1 \mathrm{H}), 5.05(\mathrm{~d}, J=3.2 \mathrm{~Hz}, 1 \mathrm{H}), 5.06(\mathrm{~d}, J=3.5 \mathrm{~Hz}, 1 \mathrm{H}), 5.23$ $(\mathrm{d}, J=2.8 \mathrm{~Hz}, 1 \mathrm{H}) ;{ }^{1} \mathrm{H} \mathrm{NMR}\left(500 \mathrm{MHz}, \mathrm{CDCl}_{3}, 1 \mathrm{D}-\mathrm{TOCSY}\right.$, irradiation at $\left.\mathrm{H} 1^{\mathrm{VI}}, 5.23 \mathrm{ppm}\right): \delta$ 3.43 (br s, $\left.1 \mathrm{H}, \mathrm{H} 2{ }^{\mathrm{VI}}\right), 3.99\left(\mathrm{dd}, J=3.2,3.2 \mathrm{~Hz}, 1 \mathrm{H}, \mathrm{H} 3^{\mathrm{VI}}\right), 4.21\left(\mathrm{~d}, J=2.8 \mathrm{~Hz}, 1 \mathrm{H}, \mathrm{H} 4^{\mathrm{VI}}\right) ;{ }^{1} \mathrm{H} \mathrm{NMR}$ (500 MHz, $\left.\mathrm{C}_{6} \mathrm{D}_{6}\right): \delta 1.89(\mathrm{~s}, 3 \mathrm{H}), 3.12(\mathrm{dd}, J=9.6,3.3 \mathrm{~Hz}, 1 \mathrm{H}), 3.15(\mathrm{dd}, J=9.8,3.2 \mathrm{~Hz}, 1 \mathrm{H})$, $3.24(\mathrm{~s}, 3 \mathrm{H}), 3.25(\mathrm{~s}, 3 \mathrm{H}), 3.28(\mathrm{~s}, 6 \mathrm{H}), 3.30(\mathrm{~s}, 3 \mathrm{H}), 3.32(\mathrm{~s}, 3 \mathrm{H}), 3.33(\mathrm{~s}, 3 \mathrm{H}), 3.36(\mathrm{~s}, 3 \mathrm{H}), 3.39$ $(\mathrm{s}, 3 \mathrm{H}), 3.42(\mathrm{~s}, 3 \mathrm{H}), 3.50(\mathrm{~s}, 3 \mathrm{H}), 3.61(\mathrm{~s}, 3 \mathrm{H}), 3.70(\mathrm{~s}, 3 \mathrm{H}), 3.73(\mathrm{~s}, 3 \mathrm{H}), 3.76(\mathrm{~s}, 3 \mathrm{H}), 3.84(\mathrm{~s}$, 3H), $3.87(\mathrm{~s}, 3 \mathrm{H}), 4.74(\mathrm{~d}, J=7.9 \mathrm{~Hz}, 1 \mathrm{H}), 5.04(\mathrm{~d}, J=3.2 \mathrm{~Hz}, 1 \mathrm{H}), 5.12(\mathrm{~d}, J=3.2 \mathrm{~Hz}, 1 \mathrm{H}), 5.19$ 
$(\mathrm{d}, J=3.5 \mathrm{~Hz}, 1 \mathrm{H}), 5.20(\mathrm{~d}, J=3.8 \mathrm{~Hz}, 1 \mathrm{H}), 5.22(\mathrm{~d}, J=3.5 \mathrm{~Hz}, 1 \mathrm{H}), 5.27(\mathrm{~d}, J=3.5 \mathrm{~Hz}, 1 \mathrm{H}) ;{ }^{1} \mathrm{H}$ NMR (500 MHz, $\mathrm{C}_{6} \mathrm{D}_{6}, 1 \mathrm{D}-\mathrm{TOCSY}$, irradiation at $\left.\mathrm{H}^{\mathrm{VI}}, 5.27 \mathrm{ppm}\right): \delta 3.49$ (dd, $J=8.5,2.8 \mathrm{~Hz}$, $\left.1 \mathrm{H}, \mathrm{H} 2^{\mathrm{VI}}\right), 3.73\left(\mathrm{dd}, J=7.9,7.9 \mathrm{~Hz}, 1 \mathrm{H}, \mathrm{H} 3^{\mathrm{VI}}\right), 4.74\left(\mathrm{~d}, J=7.9 \mathrm{~Hz}, 1 \mathrm{H}, \mathrm{H} 4{ }^{\mathrm{VI}}\right) ;{ }^{13} \mathrm{C}$ NMR $(125.7$ $\left.\mathrm{MHz}, \mathrm{CDCl}_{3}\right): \delta 21.88\left(\mathrm{CH}_{3}\right), 57.27\left(\mathrm{CH}_{3}\right), 57.74\left(\mathrm{CH}_{3}\right), 57.80\left(\mathrm{CH}_{3}\right), 58.11\left(\mathrm{CH}_{3}\right), 58.33\left(\mathrm{CH}_{3}\right)$, $58.36\left(\mathrm{CH}_{3}\right), 58.71\left(\mathrm{CH}_{3}\right), 58.82\left(\mathrm{CH}_{3}\right), 58.86\left(\mathrm{CH}_{3}\right), 58.93\left(\mathrm{CH}_{3}\right), 59.04\left(\mathrm{CH}_{3}\right), 59.08\left(\mathrm{CH}_{3}\right), 61.21$ $\left(\mathrm{CH}_{2}\right), 61.48\left(\mathrm{CH}_{3}\right), 61.62\left(\mathrm{CH}_{3}\right), 61.66\left(\mathrm{CH}_{3}\right), 61.77\left(\mathrm{CH}_{3}\right), 61.92\left(\mathrm{CH}_{3}\right), 70.84(\mathrm{CH}), 70.92\left(\mathrm{CH}_{2}\right)$, $71.14\left(\mathrm{CH}_{2}\right), 71.16(\mathrm{CH}), 71.35\left(2 \times \mathrm{CH}_{2}\right), 71.68(\mathrm{CH}), 71.79(\mathrm{CH}), 71.83\left(\mathrm{CH}_{2}\right), 72.17(\mathrm{CH})$, $74.04(\mathrm{CH}), 76.44(\mathrm{CH}), 77.31(\mathrm{CH}), 80.57(\mathrm{CH}), 81.10(\mathrm{CH}), 81.21(\mathrm{CH}), 81.41(\mathrm{CH}), 81.49$ $(\mathrm{CH}), 81.54(\mathrm{CH}), 81.57(\mathrm{CH}), 81.92(\mathrm{CH}), 82.19(\mathrm{CH}), 82.25(\mathrm{CH}), 82.33(2 \times \mathrm{CH}), 82.40(\mathrm{CH})$, $82.57(\mathrm{CH}), 82.65(\mathrm{CH}), 97.26(\mathrm{CH}), 98.28(\mathrm{CH}), 99.78(\mathrm{CH}), 99.89(\mathrm{CH}), 100.41(\mathrm{CH}), 101.39$ $(\mathrm{CH}), 103.99(\mathrm{C}), 169.76(\mathrm{C}) ;{ }^{13} \mathrm{C}$ NMR $\left(125.7 \mathrm{MHz}, \mathrm{C}_{6} \mathrm{D}_{6}\right): \delta 22.36\left(\mathrm{CH}_{3}\right), 57.35\left(\mathrm{CH}_{3}\right), 57.54$ $\left(\mathrm{CH}_{3}\right), 57.81\left(\mathrm{CH}_{3}\right), 57.83\left(\mathrm{CH}_{3}\right), 58.22\left(2 \times \mathrm{CH}_{3}\right), 58.80\left(\mathrm{CH}_{3}\right), 58.82\left(\mathrm{CH}_{3}\right), 58.93\left(\mathrm{CH}_{3}\right), 59.01$ $\left(\mathrm{CH}_{3}\right), 59.87\left(\mathrm{CH}_{3}\right), 60.41\left(\mathrm{CH}_{3}\right), 61.60\left(\mathrm{CH}_{3}\right), 61.71\left(\mathrm{CH}_{3}\right), 61.75\left(\mathrm{CH}_{3}\right), 61.79\left(\mathrm{CH}_{3}\right), 61.80$ $\left(\mathrm{CH}_{3}\right), 62.77\left(\mathrm{CH}_{2}\right), 71.94(\mathrm{CH}), 72.01(\mathrm{CH}), 72.01\left(\mathrm{CH}_{2}\right), 72.17\left(2 \times \mathrm{CH}_{2}\right), 72.31(\mathrm{CH}), 72.60$ $\left(\mathrm{CH}_{2}\right), 72.60(\mathrm{CH}), 73.22\left(\mathrm{CH}_{2}\right), 73.39(\mathrm{CH}), 79.60(\mathrm{CH}), 80.56(\mathrm{CH}), 80.96(\mathrm{CH}), 81.10(\mathrm{CH})$, $81.94(\mathrm{CH}), 81.98(\mathrm{CH}), 82.28(\mathrm{CH}), 82.39(\mathrm{CH}), 82.41(\mathrm{CH}), 82.57(2 \times \mathrm{CH}), 82.60(\mathrm{CH}), 82.73$ $(\mathrm{CH}), 82.94(\mathrm{CH}), 83.09(\mathrm{CH}), 83.16(\mathrm{CH}), 83.26(2 \times \mathrm{CH}), 98.68(\mathrm{CH}), 99.22(\mathrm{CH}), 99.58(\mathrm{CH})$, $99.99(\mathrm{CH}), 100.28(\mathrm{CH}), 100.62(\mathrm{CH}), 104.57$ (C), $169.11(\mathrm{C})$; MS (ESI+-TOF): $m / z(\%) 1291$ $\left[(\mathrm{M}+\mathrm{Na})^{+}, 100\right]$; HRMS (ESI ${ }^{+}$-TOF): $m / z$ [M $\left.+\mathrm{Na}\right]^{+}$calcd for $\mathrm{C}_{55} \mathrm{H}_{96} \mathrm{NaO}_{32}$ 1291.5782; found 1291.5819. Anal. calcd for $\mathrm{C}_{55} \mathrm{H}_{96} \mathrm{O}_{32}$ : C, 52.04; H, 7.62. Found: C, 52.25; H, 7.49.

Oxidative HAT of $2^{I-V I I I}, 3^{I-V I I I}, 6^{I I-V I I I}$-tricosa-O-methyl- $\gamma$-cyclomaltooctaose (8). Method A: A solution of alcohol $\mathbf{8}^{26}(31 \mathrm{mg}, 0.019 \mathrm{mmol})$ in dry $\mathrm{CH}_{2} \mathrm{Cl}_{2}(0.78 \mathrm{~mL})$ containing DIB (113.5 mg, $0.042 \mathrm{mmol})$ and $\mathrm{I}_{2}(4.8 \mathrm{mg}, 0.019 \mathrm{mmol})$ was stirred under nitrogen at $25{ }^{\circ} \mathrm{C}$ for $1 \mathrm{~h}$ while 
irradiated with two $80 \mathrm{~W}$ tungsten-filament lamps. An excess of solid $\mathrm{Na}_{2} \mathrm{~S}_{2} \mathrm{O}_{3}$ was then added and stirring continued until complete disappearance of the iodine color. The reaction mixture was then filtered and concentrated under reduced pressure. Silica gel [Merck 60 PF $(0.063-0.2 \mathrm{~mm})$ ] column chromatography of the reaction residue (hexanes-acetone, 60:40) afforded cyclo- $5^{\mathrm{VIII}}, 6^{\mathrm{I}}$-anhydro$\left(5^{\mathrm{VIII}} R\right)-(2,3,6-\operatorname{tri}-O$-methyl- $\alpha$-D-xylo-hexos-5-ulopyranosyl)-(1 $\rightarrow 4)-2,3-\mathrm{di}-O$-methyl- $\alpha-\mathrm{D}-$ glucopyranosyl-[(1 $\rightarrow 4)-2,3,6$-tri- $O$-methyl- $\alpha$-D-glucopyranosyl $]$ (9) (13.6 mg, 0.008 mmol, 44\%): colorless oil, $[\alpha]_{\mathrm{D}}+130.9$ (c 1.380, $\left.\mathrm{CHCl}_{3}\right)$; IR (film): 2929, 1141, 1104, $1039 \mathrm{~cm}^{-1} ;{ }^{1} \mathrm{H}$ NMR (500 $\left.\mathrm{MHz}, \mathrm{CDCl}_{3}\right): \delta 3.14-3.29(\mathrm{~m}, 8 \mathrm{H}), 3.34(\mathrm{~s}, 3 \mathrm{H}), 3.36(\mathrm{~s}, 3 \mathrm{H}), 3.37$ (s, 3H), 3.377 (s, 3H), 3.379 (s, 3H), 3.39 (s, 3H), 3.459 (s, 3H), $3.464(\mathrm{~s}, 3 \mathrm{H}), 3.47$ (s, 3H), $3.48(\mathrm{~s}, 3 \mathrm{H}), 3.491(\mathrm{~s}, 3 \mathrm{H}), 3.495$ $(\mathrm{s}, 6 \mathrm{H}), 3.50(\mathrm{~s}, 3 \mathrm{H}), 3.51(\mathrm{~s}, 3 \mathrm{H}), 3.54(\mathrm{~s}, 3 \mathrm{H}), 3.58(\mathrm{~s}, 3 \mathrm{H}), 3.59(\mathrm{~s}, 3 \mathrm{H}), 3.603(\mathrm{~s}, 3 \mathrm{H}), 3.606(\mathrm{~s}$, 3H), $3.611(\mathrm{~s}, 3 \mathrm{H}), 3.639(\mathrm{~s}, 3 \mathrm{H}), 3.643(\mathrm{~s}, 3 \mathrm{H}), 5.08(\mathrm{~d}, J=3.8 \mathrm{~Hz}, 1 \mathrm{H}), 5.18(\mathrm{~d}, J=3.2 \mathrm{~Hz}, 1 \mathrm{H})$, $5.20(\mathrm{~d}, J=3.8 \mathrm{~Hz}, 1 \mathrm{H}), 5.21(\mathrm{~d}, J=3.8 \mathrm{~Hz}, 1 \mathrm{H}), 5.22(\mathrm{~d}, J=4.1 \mathrm{~Hz}, 1 \mathrm{H}), 5.24(\mathrm{~d}, J=2.2 \mathrm{~Hz}$, $1 \mathrm{H}), 5.25(\mathrm{~d}, J=2.8 \mathrm{~Hz}, 1 \mathrm{H}), 5.58(\mathrm{~d}, J=3.5 \mathrm{~Hz}, 1 \mathrm{H}) ;{ }^{13} \mathrm{C} \mathrm{NMR}\left(125.7 \mathrm{MHz}, \mathrm{CDCl}_{3}\right): \delta 57.46$ $\left(\mathrm{CH}_{3}\right), 57.74\left(\mathrm{CH}_{3}\right), 58.34\left(\mathrm{CH}_{3}\right), 58.41\left(\mathrm{CH}_{3}\right), 58.48\left(\mathrm{CH}_{3}\right), 58.51\left(\mathrm{CH}_{3}\right), 58.85\left(2 \times \mathrm{CH}_{3}\right), 58.88$ $\left(\mathrm{CH}_{3}\right), 58.93\left(2 \times \mathrm{CH}_{3}\right), 58.96\left(\mathrm{CH}_{3}\right), 58.99\left(\mathrm{CH}_{3}\right), 59.20\left(2 \times \mathrm{CH}_{3}\right), 59.60\left(\mathrm{CH}_{3}\right), 59.99\left(\mathrm{CH}_{3}\right)$, $60.37\left(\mathrm{CH}_{3}\right), 60.98\left(\mathrm{CH}_{3}\right), 61.02\left(2 \times \mathrm{CH}_{3}\right), 61.20\left(\mathrm{CH}_{3}\right), 61.70\left(\mathrm{CH}_{3}\right), 64.44\left(\mathrm{CH}_{2}\right), 66.25(\mathrm{CH})$, $69.00(\mathrm{CH}), 69.90(\mathrm{CH}), 70.18(\mathrm{CH}), 70.22\left(\mathrm{CH}_{2}\right), 70.55(\mathrm{CH}), 70.78(\mathrm{CH}), 70.91(\mathrm{CH}), 71.04$ $\left(\mathrm{CH}_{2}\right), 71.08\left(2 \times \mathrm{CH}_{2}\right), 71.21(\mathrm{CH}), 71.64\left(\mathrm{CH}_{2}\right), 71.94\left(\mathrm{CH}_{2}\right), 72.18\left(\mathrm{CH}_{2}\right), 74.47(\mathrm{CH}), 77.13$ $(\mathrm{CH}), 77.62(\mathrm{CH}), 77.92(\mathrm{CH}), 78.05(\mathrm{CH}), 78.58(\mathrm{CH}), 79.62(\mathrm{CH}), 79.72(\mathrm{CH}), 80.67(\mathrm{CH})$, $81.17(\mathrm{CH}), 81.58(\mathrm{CH}), 81.63(\mathrm{CH}), 81.69(3 \times \mathrm{CH}), 81.81(\mathrm{CH}), 82.28(\mathrm{CH}), 82.31(\mathrm{CH}), 82.46$ $(\mathrm{CH}), 82.61(\mathrm{CH}), 82.76(\mathrm{CH}), 83.02(\mathrm{CH}), 83.18(\mathrm{CH}), 92.88(\mathrm{CH}), 95.20(\mathrm{CH}), 96.85(\mathrm{CH})$, $97.59(\mathrm{CH}), 97.84(\mathrm{CH}), 98.35(\mathrm{CH}), 98.82(\mathrm{CH}), 99.55(\mathrm{CH}), 100.64(\mathrm{C}) ; \mathrm{MS}\left(\mathrm{ESI}^{+}-\mathrm{TOF}\right): m / z$ 
(\%) $1639\left[(\mathrm{M}+\mathrm{Na})^{+}, 100\right]$; HRMS (ESI $\left.-\mathrm{TOF}\right): \mathrm{m} / z[\mathrm{M}+\mathrm{Na}]^{+}$calcd for $\mathrm{C}_{71} \mathrm{H}_{124} \mathrm{NaO}_{40}$ 1639.7567; found 1639.7531. Anal. calcd for $\mathrm{C}_{71} \mathrm{H}_{124} \mathrm{O}_{40}$ : C, 52.71; H, 7.73. Found: $\mathrm{C}, 52.86 ; \mathrm{H}, 7.55$.

Method B: A solution of alcohol 8 ( $45 \mathrm{mg}, 0.028 \mathrm{mmol})$ in dry $\mathrm{CH}_{2} \mathrm{Cl}_{2}(1.2 \mathrm{~mL})$ containing DIB (20 mg, $0.062 \mathrm{mmol})$ and $\mathrm{I}_{2}(3.6 \mathrm{mg}, 0.014 \mathrm{mmol})$ was stirred under nitrogen at $24{ }^{\circ} \mathrm{C}$ for $2.5 \mathrm{~h}$ while irradiated with two $80 \mathrm{~W}$ tungsten-filament lamps. The reaction mixture was then directly loaded onto a silica gel (TLC Silica gel $60 \mathrm{~F}_{254}$, scraped from Merck Aluminum sheets) column chromatography (hexanes-acetone, $65: 35)$ to give $\mathbf{9}(15.6 \mathrm{mg}, 0.01 \mathrm{mmol}, 36 \%)$ and cyclo- $(5 R)-5^{\mathrm{VIII}}$ $O$-acetyl-2,3,6-tri- $O$-methyl- $\alpha$-D-xylo-hexos-5-ulopyranosyl-( $1 \rightarrow 4)$-2,3-di- $O$-methyl- $\alpha$-Dglucopyranosyl-[(1 $\rightarrow 4)-2,3,6$-tri- $O$-methyl- $\alpha$-D-glucopyranosyl $]_{6}(\mathbf{1 0})(19.2 \mathrm{mg}, 0.011 \mathrm{mmol}$, 41\%). Compound 10: colorless oil, $[\alpha]_{\mathrm{D}}+131.6$ ( $c$ 0.91, $\left.\mathrm{CHCl}_{3}\right)$; IR (film): 3539, 2925, 1738, 1152, 1100, $1037 \mathrm{~cm}^{-1} ;{ }^{1} \mathrm{H}$ NMR (500 MHz, $\left.\mathrm{CDCl}_{3}\right): \delta 2.06(\mathrm{~s}, 3 \mathrm{H}), 3.16(\mathrm{dd}, J=9.5,3.2 \mathrm{~Hz}, 1 \mathrm{H}), 3.19$ (dd, $J=9.8,3.5 \mathrm{~Hz}, 1 \mathrm{H}), 3.19(\mathrm{dd}, J=9.8,3.5 \mathrm{~Hz}, 1 \mathrm{H}), 3.19(\mathrm{dd}, J=9.8,3.5 \mathrm{~Hz}, 1 \mathrm{H}), 3.21(\mathrm{dd}, J$ $=9.6,3.6 \mathrm{~Hz}, 1 \mathrm{H}), 3.23(\mathrm{dd}, J=9.5,3.8 \mathrm{~Hz}, 1 \mathrm{H}), 3.26(\mathrm{dd}, J=9.8,3.8 \mathrm{~Hz}, 1 \mathrm{H}), 3.330(\mathrm{~s}, 3 \mathrm{H})$, 3.334 (s, 3H), 3.34 (s, 3H), 3.36 (s, 3H), 3.369 (s, 3H), 3.374 (s, 3H), 3.40 (s, 3H), 3.42 (s, 3H), $3.44\left(\operatorname{br~d}, J=3.9 \mathrm{~Hz}, 1 \mathrm{H}, \mathrm{H} 2^{\mathrm{VIII}}\right.$ ), 3.473 (s, 6H), 3.476 (s, 3H), 3.477 (s, 3H), 3.51 (s, 3H), 3.556 (s, 3H), $3.558(\mathrm{~s}, 3 \mathrm{H}), 3.58(\mathrm{~s}, 3 \mathrm{H}), 3.60(\mathrm{~s}, 6 \mathrm{H}), 3.62$ (s, 3H), 3.64 (s, 3H), 3.649 (s, 3H), $3.654(\mathrm{~s}$, 3H), $3.71(\mathrm{~s}, 3 \mathrm{H}), 3.99(\mathrm{~d}, J=9.8 \mathrm{~Hz}, 1 \mathrm{H}), 4.025(\mathrm{~d}, J=9.8 \mathrm{~Hz}, 1 \mathrm{H}), 4.03\left(\mathrm{br} \mathrm{s}, 1 \mathrm{H}, \mathrm{H} 4{ }^{\mathrm{VIII}}\right), 4.24$ (dd, $\left.J=2.2,2.2 \mathrm{~Hz}, 1 \mathrm{H}, \mathrm{H} 3^{\mathrm{VIII}}\right), 5.01(\mathrm{~d}, J=3.5 \mathrm{~Hz}, 1 \mathrm{H}), 5.08(\mathrm{~d}, J=3.2 \mathrm{~Hz}, 1 \mathrm{H}), 5.09(\mathrm{~d}, J=3.5$ $\mathrm{Hz}, 1 \mathrm{H}), 5.12(\mathrm{~d}, J=3.8 \mathrm{~Hz}, 1 \mathrm{H}), 5.18(\mathrm{~d}, J=3.8 \mathrm{~Hz}, 1 \mathrm{H}), 5.19(\mathrm{~d}, J=3.5 \mathrm{~Hz}, 1 \mathrm{H}), 5.23(\mathrm{~d}, J=$ $\left.1.6 \mathrm{~Hz}, 1 \mathrm{H}, \mathrm{H} 1^{\mathrm{VIII}}\right), 5.60(\mathrm{~d}, J=4.1 \mathrm{~Hz}, 1 \mathrm{H}) ;{ }^{1} \mathrm{H} \mathrm{NMR}\left(500 \mathrm{MHz}, \mathrm{CDCl}_{3}, 1 \mathrm{D}-\mathrm{TOCSY}\right.$, irradiation at $\left.\mathrm{H} 1^{\mathrm{II}}, 5.23 \mathrm{ppm}\right): \delta 3.45\left(\mathrm{~d}, J=3.9 \mathrm{~Hz}, 1 \mathrm{H}, \mathrm{H} 2^{\mathrm{VIII}}\right), 4.03\left(\mathrm{br} \mathrm{s}, 1 \mathrm{H}, \mathrm{H} 4^{\mathrm{VIII}}\right), 4.25(\mathrm{dd}, J=2.4,2.4$ $\left.\mathrm{Hz}, 1 \mathrm{H}, \mathrm{H} 3{ }^{\mathrm{VIIII}}\right) ;{ }^{1} \mathrm{H}$ NMR (500 MHz, $\left.\mathrm{C}_{6} \mathrm{D}_{6}\right): \delta 1.77(\mathrm{~s}, 3 \mathrm{H}), 3.10(\mathrm{dd}, J=9.5,2.8 \mathrm{~Hz}, 1 \mathrm{H}), 3.13$ (dd, $J=9.9,3.3 \mathrm{~Hz}, 1 \mathrm{H}), 3.19(\mathrm{~s}, 3 \mathrm{H}), 3.21(\mathrm{~s}, 3 \mathrm{H}), 3.22(\mathrm{~s}, 3 \mathrm{H}), 3.27$ (s, 3H), $3.29(\mathrm{~m}, 6 \mathrm{H}), 3.296$ 
(s, 3H), 3.304 (s, 3H), 3.31 (s, 3H), 3.322 (s, 3H), 3.325 (s, 3H), 3.35 (s, 3H), 3.36 (s, 3H), 3.37 (s, 3H), 3.39 (s, 3H), $3.46(\mathrm{~s}, 3 \mathrm{H}), 3.59$ (s, 3H), 3.65 (s, 3H), $3.70(\mathrm{~s}, 3 \mathrm{H}), 3.72(\mathrm{~s}, 3 \mathrm{H}), 3.726(\mathrm{~s}, 3 \mathrm{H})$, $3.733(\mathrm{~s}, 3 \mathrm{H}), 3.81(\mathrm{~s}, 3 \mathrm{H}), 4.30(\mathrm{dd}, J=2.5,2.5 \mathrm{~Hz}, 1 \mathrm{H}), 4.32(\mathrm{br} \mathrm{s}), 4.40(\mathrm{~d}, J=9.8 \mathrm{~Hz}, 1 \mathrm{H}), 4.46$ (br d, $J=12.0 \mathrm{~Hz}, 1 \mathrm{H}), 4.51(\mathrm{~d}, J=9.8 \mathrm{~Hz}, 1 \mathrm{H}), 5.16(\mathrm{~d}, J=3.2 \mathrm{~Hz}, 1 \mathrm{H}), 5.17(\mathrm{~d}, J=3.2 \mathrm{~Hz}, 1 \mathrm{H})$, $5.29(\mathrm{~d}, J=3.5 \mathrm{~Hz}, 1 \mathrm{H}), 5.36(\mathrm{~d}, J=3.8 \mathrm{~Hz}, 1 \mathrm{H}), 5.37(\mathrm{~d}, J=3.8 \mathrm{~Hz}, 1 \mathrm{H}), 5.57(\mathrm{~d}, J=3.8 \mathrm{~Hz}$, $1 \mathrm{H}), 5.61(\mathrm{~d}, J=3.8 \mathrm{~Hz}, 1 \mathrm{H}), 5.65(\mathrm{~d}, J=1.6 \mathrm{~Hz}, 1 \mathrm{H}) ;{ }^{13} \mathrm{C} \mathrm{NMR}\left(125.7 \mathrm{MHz}, \mathrm{CDCl}_{3}\right): \delta 22.11$ $\left(\mathrm{CH}_{3}\right), 57.40\left(\mathrm{CH}_{3}\right), 58.07\left(\mathrm{CH}_{3}\right), 58.18\left(\mathrm{CH}_{3}\right), 58.38\left(\mathrm{CH}_{3}\right), 58.40\left(\mathrm{CH}_{3}\right), 58.46\left(\mathrm{CH}_{3}\right), 58.60$ $\left(\mathrm{CH}_{3}\right), 58.84\left(\mathrm{CH}_{3}\right), 58.88\left(\mathrm{CH}_{3}\right), 58.96\left(4 \times \mathrm{CH}_{3}\right), 59.10\left(\mathrm{CH}_{3}\right), 59.64\left(2 \times \mathrm{CH}_{3}\right), 60.12\left(\mathrm{CH}_{3}\right)$, $60.22\left(\mathrm{CH}_{2}\right), 61.02\left(\mathrm{CH}_{3}\right), 61.33\left(\mathrm{CH}_{3}\right), 61.39\left(\mathrm{CH}_{3}\right), 61.46\left(\mathrm{CH}_{3}\right), 61.55\left(\mathrm{CH}_{3}\right), 61.70\left(\mathrm{CH}_{3}\right), 70.18$ $(\mathrm{CH}), 70.42\left(\mathrm{CH}_{2}\right), 70.60(\mathrm{CH}), 70.71\left(\mathrm{CH}_{2}\right), 70.78(\mathrm{CH}), 70.88\left(\mathrm{CH}_{2}\right), 70.92\left(\mathrm{CH}_{2}\right), 70.99\left(\mathrm{CH}_{2}\right)$, $71.12(\mathrm{CH}), 71.12\left(\mathrm{CH}_{2}\right), 71.21(2 \times \mathrm{CH}), 71.58(\mathrm{CH}), 71.68\left(\mathrm{CH}_{2}\right), 72.36(\mathrm{CH}), 72.48(\mathrm{CH}), 75.45$ $(\mathrm{CH}), 76.33(\mathrm{CH}), 78.79(\mathrm{CH}), 78.99(\mathrm{CH}), 79.35(\mathrm{CH}), 80.43(\mathrm{CH}), 80.45(\mathrm{CH}), 81.37(\mathrm{CH})$, $81.57(3 \times \mathrm{CH}), 81.74(2 \times \mathrm{CH}), 81.97(\mathrm{CH}), 82.04(\mathrm{CH}), 82.07(\mathrm{CH}), 82.33(2 \times \mathrm{CH}), 82.38(\mathrm{CH})$, $82.57(\mathrm{CH}), 82.60(\mathrm{CH}), 82.99(\mathrm{CH}), 96.50(\mathrm{CH}), 97.53(\mathrm{CH}), 97.85(\mathrm{CH}), 98.07(\mathrm{CH}), 98.71$ (CH), $99.05(\mathrm{CH}), 100.00(\mathrm{CH}), 101.56(\mathrm{CH}), 105.34(\mathrm{C}), 170.23(\mathrm{C})$; MS (ESI $\left.{ }^{+}-\mathrm{TOF}\right): \mathrm{m} / z(\%)$ $1699\left[(\mathrm{M}+\mathrm{Na})^{+}, 100\right] ; \mathrm{HRMS}\left(\mathrm{ESI}^{+}-\mathrm{TOF}\right): \mathrm{m} / z[\mathrm{M}+\mathrm{Na}]^{+}$calcd for $\mathrm{C}_{73} \mathrm{H}_{128} \mathrm{NaO}_{42}$ 1699.7778; found 1699.7756. Anal. calcd for $\mathrm{C}_{73} \mathrm{H}_{128} \mathrm{O}_{42}$ : C, 52.26; H, 7.69. Found: $\mathrm{C}, 52.15 ; \mathrm{H}, 7.53$. $2^{I-V I}, 3^{I-V I}, 6^{I I, I I I, V, V I}$-Hexadeca-O-methyl- $\alpha$-cyclomaltohexaose (14), $2^{I-V I}, 3^{I-V I}, 6^{I I, I V-V I}$-HexadecaO-methyl- $\alpha$-cyclomaltohexaose (23), and $\quad 2^{I-V I}, 3^{I-V I}, 6^{I I I-V I}-H e x a d e c a-O$-methyl- $\alpha$ cyclomaltohexaose (36). A solution of dry a-cyclodextrin $(4.69 \mathrm{~g}, 4.825 \mathrm{mmol})$ and imidazole $(1.148 \mathrm{~g}, 16.9 \mathrm{mmol})$ in dry DMF $(246 \mathrm{~mL})$ was added TBDMSCl $(2.654 \mathrm{~g}, 9.7 \mathrm{mmol})$ in one portion at room temperature under nitrogen and the mixture stirred at this temperature for $2 \mathrm{~h}$. After cooling at $0{ }^{\circ} \mathrm{C}, \mathrm{NaH}(60 \%, 10.615 \mathrm{~g}, 265 \mathrm{mmol})$ was added in small portions and the mixture 
stirred for $30 \mathrm{~min}$ and then at room temperature for $1 \mathrm{~h}$. After recooling to $0{ }^{\circ} \mathrm{C}$, MeI $(34.2 \mathrm{~mL}, 549$ mmol) was then added dropwise and the stirring continued at room temperature overnight. The excess of $\mathrm{NaH}$ was destroyed with $\mathrm{MeOH}$ and the mixture poured into ice-water and extracted with $\mathrm{CHCl}_{3}(5 \times 100 \mathrm{~mL})$. The organic phase was dried over $\mathrm{Na}_{2} \mathrm{SO}_{4}$ and concentrated under reduced pressure. The residue in dry $\mathrm{MeOH}(290 \mathrm{~mL})$ was treated with $\mathrm{NH}_{4} \mathrm{~F}(6.28 \mathrm{~g}, 170 \mathrm{mmol})$ and heated at reflux temperature under nitrogen for $22.5 \mathrm{~h}$. The solvent was evaporated under reduced pressure and the residue redissolved in $\mathrm{CHCl}_{3}(200 \mathrm{~mL})$, filtered over Celite and concentrated to give a crude mixture $(12.15 \mathrm{~g})$ that was purified by column chromatography $\left(\mathrm{CHCl}_{3} \rightarrow \mathrm{CHCl}_{3}-\mathrm{MeOH}\right.$, 96:4) to give the known permethylated $\alpha-C D(628 \mathrm{mg}, 0.513 \mathrm{mmol}, 10 \%), 2^{\mathrm{I}-\mathrm{VI}}, 3^{\mathrm{I}-\mathrm{VI}}, 6^{\mathrm{II}-\mathrm{VI}}-$ heptadeca- $O$-methyl- $\alpha$-cyclomaltohexaose $(5)^{25}(1602 \mathrm{mg}, 1.324 \mathrm{mmol}, 27 \%)$, and a mixture of the three possible diols that was subjected to a second careful flash chromatography on 60 PF silica gel (0.04-0.063) $\left(\mathrm{CHCl}_{3}-\mathrm{MeOH}, 98: 2 \rightarrow 95: 5\right)$ to give the diols $14^{25,29}$ (385 $\left.\mathrm{mg}, 0.322 \mathrm{mmol}, 7 \%\right)$, 23 (731 mg, $0.611 \mathrm{mmol}, 13 \%)$, and $36^{35}$ (936 mg, $\left.0.783 \mathrm{mmol}, 16 \%\right)$. A more polar fraction containing a mixture of triols and other polyols was not studied. Compound 23: colorless oil, $[\alpha]_{\mathrm{D}}$ +141.2 (c 0.97, $\mathrm{CHCl}_{3}$ ); IR (film): 3472, 2929, 1454, 1365, 1107, $1039 \mathrm{~cm}^{-1} ;{ }^{1} \mathrm{H} \mathrm{NMR}(500 \mathrm{MHz}$, $\left.\mathrm{CDCl}_{3}\right): \delta 3.13-3.19(\mathrm{~m}, 6 \mathrm{H}), 3.389(\mathrm{~s}, 3 \mathrm{H}), 3.397(\mathrm{~s}, 3 \mathrm{H}), 3.398(\mathrm{~s}, 6 \mathrm{H}), 3.489(\mathrm{~s}, 3 \mathrm{H}), 3.492(\mathrm{~s}$, 3H), $3.495(\mathrm{~s}, 6 \mathrm{H}), 3.50(\mathrm{~s}, 6 \mathrm{H}), 3.63(\mathrm{~s}, 3 \mathrm{H}), 3.647(\mathrm{~s}, 6 \mathrm{H}), 3.649(\mathrm{~s}, 3 \mathrm{H}), 3.650(\mathrm{~s}, 3 \mathrm{H}), 3.66(\mathrm{~s}$, $3 \mathrm{H}), 5.03(\mathrm{~d}, J=3.8 \mathrm{~Hz}, 1 \mathrm{H}), 5.035(\mathrm{~d}, J=3.8 \mathrm{~Hz}, 1 \mathrm{H}), 5.05(\mathrm{~d}, J=3.5 \mathrm{~Hz}, 1 \mathrm{H}), 5.06(\mathrm{~d}, J=3.5$ $\mathrm{Hz}, 1 \mathrm{H}), 5.07(\mathrm{~d}, J=3.5 \mathrm{~Hz}, 1 \mathrm{H}), 5.08(\mathrm{~d}, J=3.5 \mathrm{~Hz}, 1 \mathrm{H}) ;{ }^{13} \mathrm{C} \mathrm{NMR}\left(125.7 \mathrm{MHz}, \mathrm{CDCl}_{3}\right): \delta 57.85$ $\left(2 \times \mathrm{CH}_{3}\right), 57.90\left(2 \times \mathrm{CH}_{3}\right), 58.03\left(\mathrm{CH}_{3}\right), 58.08\left(\mathrm{CH}_{3}\right), 58.93\left(\mathrm{CH}_{3}\right), 59.01\left(\mathrm{CH}_{3}\right), 59.09\left(\mathrm{CH}_{3}\right)$, $59.15\left(\mathrm{CH}_{3}\right), 61.63\left(2 \times \mathrm{CH}_{3}\right), 61.75\left(2 \times \mathrm{CH}_{3}\right), 61.77\left(\mathrm{CH}_{3}\right), 61.79\left(\mathrm{CH}_{3}\right), 62.32\left(\mathrm{CH}_{2}\right), 62.40$ $\left(\mathrm{CH}_{2}\right), 71.19(\mathrm{CH}), 71.28(2 \times \mathrm{CH}), 71.43\left(\mathrm{CH}_{2}\right), 71.51(\mathrm{CH}), 71.53\left(\mathrm{CH}_{2}\right), 71.55\left(\mathrm{CH}_{2}\right), 71.69$ $\left(\mathrm{CH}_{2}\right), 72.48(\mathrm{CH}), 72.65(\mathrm{CH}), 81.24(\mathrm{CH}), 81.28(3 \times \mathrm{CH}), 81.35(\mathrm{CH}), 81.40(\mathrm{CH}), 81.74(\mathrm{CH})$, 
$81.97(\mathrm{CH}), 82.00(\mathrm{CH}), 82.06(2 \times \mathrm{CH}), 82.12(\mathrm{CH}), 82.17(2 \times \mathrm{CH}), 82.22(2 \times \mathrm{CH}), 82.29(\mathrm{CH})$, $82.35(\mathrm{CH}), 99.36(\mathrm{CH}), 99.58(\mathrm{CH}), 99.84(\mathrm{CH}), 99.87(2 \times \mathrm{CH}), 99.90(\mathrm{CH}) ; \mathrm{MS}\left(\mathrm{ESI}^{+}-\mathrm{TOF}\right)$ : $m / z(\%) 1214\left[\left(\mathrm{M}+\mathrm{NH}_{4}\right)^{+}, 100\right] ; \mathrm{HRMS}\left(\mathrm{ESI}^{+}-\mathrm{TOF}\right): m / z\left[\mathrm{M}+\mathrm{NH}_{4}\right]^{+}$calcd for $\mathrm{C}_{52} \mathrm{H}_{96} \mathrm{NO}_{30}$ 1214.6017; found 1214.6023. Anal. calcd for $\mathrm{C}_{52} \mathrm{H}_{92} \mathrm{O}_{30}$ : C, 52.17; H, 7.75. Found: $\mathrm{C}, 52.05 ; \mathrm{H}$, 7.61. Compound 36: colorless oil, $[\alpha]_{\mathrm{D}}+143.3$ (c 1.21, $\left.\mathrm{CHCl}_{3}\right)$; IR (film): 3468, 2933, 1456, 1365, 1109, $1037 \mathrm{~cm}^{-1} ;{ }^{1} \mathrm{H}$ NMR (500 MHz, $\left.\mathrm{CDCl}_{3}\right): \delta 3.12-3.19(\mathrm{~m}, 6 \mathrm{H}), 3.386(\mathrm{~s}, 6 \mathrm{H}), 3.390(\mathrm{~s}, 6 \mathrm{H})$, 3.477 (s, 3H), 3.485 (s, 6H), $3.492(\mathrm{~s}, 3 \mathrm{H}), 3.497$ (s, 3H), $3.501(\mathrm{~s}, 3 \mathrm{H}), 3.632(\mathrm{~s}, 3 \mathrm{H}), 3.637$ (s, 3H), $3.640(\mathrm{~s}, 3 \mathrm{H}), 3.642(\mathrm{~s}, 3 \mathrm{H}), 3.644(\mathrm{~s}, 3 \mathrm{H}), 3.65(\mathrm{~s}, 3 \mathrm{H}), 5.01(\mathrm{~d}, J=3.5 \mathrm{~Hz}, 1 \mathrm{H}), 5.04(\mathrm{~d}, J=$ $3.2 \mathrm{~Hz}, 4 \mathrm{H}), 5.08(\mathrm{~d}, J=3.5 \mathrm{~Hz}, 1 \mathrm{H}) ;{ }^{13} \mathrm{C} \mathrm{NMR}\left(125.7 \mathrm{MHz}, \mathrm{CDCl}_{3}\right): \delta 57.85\left(\mathrm{CH}_{3}\right), 57.90(3 \times$ $\left.\mathrm{CH}_{3}\right), 57.97\left(2 \times \mathrm{CH}_{3}\right), 58.94\left(2 \times \mathrm{CH}_{3}\right), 59.00\left(\mathrm{CH}_{3}\right), 59.09\left(\mathrm{CH}_{3}\right), 61.67\left(\mathrm{CH}_{3}\right), 61.72\left(3 \times \mathrm{CH}_{3}\right)$, $61.77\left(2 \times \mathrm{CH}_{3}\right), 62.20\left(\mathrm{CH}_{2}\right), 62.54\left(\mathrm{CH}_{2}\right), 71.14(\mathrm{CH}), 71.17(\mathrm{CH}), 71.25(2 \times \mathrm{CH}), 71.44\left(\mathrm{CH}_{2}\right)$, $71.51\left(2 \times \mathrm{CH}_{2}\right), 71.60\left(\mathrm{CH}_{2}\right), 72.59(\mathrm{CH}), 72.98(\mathrm{CH}), 81.26(2 \times \mathrm{CH}), 81.30(4 \times \mathrm{CH}), 81.99(3$ $\times \mathrm{CH}), 82.06(2 \times \mathrm{CH}), 82.15(2 \times \mathrm{CH}), 82.16(\mathrm{CH}), 82.22(\mathrm{CH}), 82.26(\mathrm{CH}), 82.45(\mathrm{CH}), 82.58$ (CH), $99.58(\mathrm{CH}), 99.66(\mathrm{CH}), 99.76(\mathrm{CH}), 99.86(\mathrm{CH}), 99.89(\mathrm{CH}), 99.94(\mathrm{CH})$; MS (ESI $\left.{ }^{+}-\mathrm{TOF}\right)$ : $m / z(\%) 1219\left[(\mathrm{M}+\mathrm{Na})^{+}, 100\right]$; HRMS $\left(\mathrm{ESI}^{+}-\mathrm{TOF}\right): m / z[\mathrm{M}+\mathrm{Na}]^{+}$calcd for $\mathrm{C}_{52} \mathrm{H}_{92} \mathrm{NaO}_{30}$ 1219.5571; found 1219.5576. Anal. calcd for $\mathrm{C}_{52} \mathrm{H}_{92} \mathrm{O}_{30}$ : C, 52.17; H, 7.75. Found: C, 52.30; H, 7.74 .

Oxidative HAT of $2^{I-V I I}, 3^{I-V I I}, 6^{I I, I I, V-V I I}$-nonadeca-O-methyl- $\beta$-cyclomaltoheptaose (11). A solution of alcohol $11^{17 \mathrm{a}}(80 \mathrm{mg}, 0.057 \mathrm{mmol})$ in dry $\mathrm{CH}_{2} \mathrm{Cl}_{2}(2.3 \mathrm{~mL})$ containing DIB (55 mg, $0.171 \mathrm{mmol})$ and $\mathrm{I}_{2}(24.6 \mathrm{mg}, 0.097 \mathrm{mmol})$ was stirred under nitrogen at $30{ }^{\circ} \mathrm{C}$ for $1 \mathrm{~h}$ while irradiated with two $80 \mathrm{~W}$ tungsten-filament lamps. The reaction mixture was then poured into $10 \%$ aqueous $\mathrm{Na}_{2} \mathrm{~S}_{2} \mathrm{O}_{3}$, extracted with $\mathrm{CH}_{2} \mathrm{Cl}_{2}$, dried over $\mathrm{Na}_{2} \mathrm{SO}_{4}$, and concentrated. The residue was purified by silica gel [Merck $60 \mathrm{PF}(0.063-0.2 \mathrm{~mm})]$ column chromatography (hexanes-acetone, 
$65: 35 \rightarrow 50: 50) \quad$ to give cyclo- $5^{\mathrm{VII}}, 6^{\mathrm{I}}$-anhydro- $\left(5^{\mathrm{VII}} R\right)-(2,3,6$-tri- $O$-methyl- $\alpha$-D-xylo-hexos-5ulopyranosyl)-(1 $\rightarrow 4)$-2,3-di-O-methyl- $\alpha$-D-glucopyranosyl-( $1 \rightarrow 4)$-2,3,6-tri- $O$-methyl- $\alpha$-Dglucopyranosyl-( $(1 \rightarrow 4)-5^{\mathrm{III}}, 6^{\mathrm{IV}}$-anhydro-( $\left({ }^{\mathrm{III}} R\right)-(2,3,6$-tri- $O$-methyl- $\alpha$-D-xylo-hexos-5ulopyranosyl)-(1 $\rightarrow 4)$-2,3-di-O-methyl- $\alpha$-D-glucopyranosyl-[( $1 \rightarrow 4)$-2,3,6-tri- $O$-methyl- $\alpha$-Dglucopyranosyl] 2 (12) (16.2 $\mathrm{mg}, 0.012 \mathrm{mmol}, 20 \%)$, an inseparable mixture of mono-trioxocanemono-acetyl positional isomers $(11.9 \mathrm{mg}, 0.008 \mathrm{mmol}, 3: 2,14 \%)$ and cyclo- $(5 R)-5^{\mathrm{VII}}-O$-acetyl-2,3,6tri-O-methyl- $\alpha$-D-xylo-hexos-5-ulopyranosyl-( $(\rightarrow 4)$-2,3-di- $O$-methyl- $\alpha$-D-glucopyranosyl$(1 \rightarrow 4)$-2,3,6-tri- $O$-methyl- $\alpha$-D-glucopyranosyl-( $(1 \rightarrow 4)-(5 R)-5^{\mathrm{III}}-O$-acetyl-2,3,6-tri- $O$-methyl- $\alpha$-Dxylo-hexos-5-ulopyranosyl-( $1 \rightarrow 4)$-2,3-di- $O$-methyl- $\alpha$-D-glucopyranosyl-[(1 $\rightarrow 4)-2,3,6$-tri- $O$ methyl- $\alpha$-D-glucopyranosyl $]_{2}$ (13) (42.3 mg, $\left.0.028 \mathrm{mmol}, 49 \%\right)$. Compound 12: colorless oil, $[\alpha]_{\mathrm{D}}$ +110.9 (c 0.79, $\mathrm{CHCl}_{3}$ ); IR (film): 2933, 1454, 1365, 1139, 1065, $1044 \mathrm{~cm}^{-1} ;{ }^{1} \mathrm{H}$ NMR (500 MHz, $\left.\mathrm{CDCl}_{3}\right): \delta 3.11(\mathrm{dd}, J=9.8,3.2 \mathrm{~Hz}, 1 \mathrm{H}), 3.13(\mathrm{dd}, J=9.5,3.2 \mathrm{~Hz}, 1 \mathrm{H}), 3.179(\mathrm{dd}, J=9.5,3.5 \mathrm{~Hz}$, $1 \mathrm{H}), 3.181(\mathrm{dd}, J=9.5,3.5 \mathrm{~Hz}, 1 \mathrm{H}), 3.21(\mathrm{dd}, J=9.8,3.8 \mathrm{~Hz}, 1 \mathrm{H}), 3.28(\mathrm{dd}, J=9.5,3.2 \mathrm{~Hz}, 1 \mathrm{H})$, $3.28(\mathrm{dd}, J=9.5,3.2 \mathrm{~Hz}, 1 \mathrm{H}), 3.37$ (s, 3H), $3.378(\mathrm{~s}, 3 \mathrm{H}), 3.380(\mathrm{~s}, 6 \mathrm{H}), 3.39$ (s, 3H), $3.465(\mathrm{~s}$, 3H), 3.468 (s, 3H), 3.47 (s, 3H), $3.478(\mathrm{~s}, 3 \mathrm{H}), 3.484(\mathrm{~s}, 3 \mathrm{H}), 3.50(\mathrm{~s}, 3 \mathrm{H}), 3.52(\mathrm{~s}, 3 \mathrm{H}), 3.59(\mathrm{~s}$, 3H), $3.601(\mathrm{~s}, 3 \mathrm{H}), 3.604(\mathrm{~s}, 3 \mathrm{H}), 3.62(\mathrm{~s}, 3 \mathrm{H}), 3.63(\mathrm{~s}, 3 \mathrm{H}), 3.67(\mathrm{~s}, 3 \mathrm{H}), 3.69(\mathrm{~s}, 3 \mathrm{H}), 5.02(\mathrm{~d}, J=$ $3.5 \mathrm{~Hz}, 1 \mathrm{H}), 5.04(\mathrm{~d}, J=2.8 \mathrm{~Hz}, 1 \mathrm{H}), 5.053(\mathrm{~d}, J=3.5 \mathrm{~Hz}, 1 \mathrm{H}), 5.058(\mathrm{~d}, J=3.8 \mathrm{~Hz}, 1 \mathrm{H}), 5.066$ $(\mathrm{d}, J=4.1 \mathrm{~Hz}, 1 \mathrm{H}), 5.21(\mathrm{~d}, J=3.8 \mathrm{~Hz}, 1 \mathrm{H}), 5.22(\mathrm{~d}, J=3.5 \mathrm{~Hz}, 1 \mathrm{H}) ;{ }^{13} \mathrm{C} \mathrm{NMR}(125.7 \mathrm{MHz}$, $\left.\mathrm{CDCl}_{3}\right): \delta 57.74\left(\mathrm{CH}_{3}\right), 57.94\left(2 \times \mathrm{CH}_{3}\right), 58.04\left(\mathrm{CH}_{3}\right), 58.08\left(\mathrm{CH}_{3}\right), 58.33\left(\mathrm{CH}_{3}\right), 58.43\left(\mathrm{CH}_{3}\right)$, $58.95\left(4 \times \mathrm{CH}_{3}\right), 59.06\left(\mathrm{CH}_{3}\right), 61.09\left(2 \times \mathrm{CH}_{3}\right), 61.66\left(\mathrm{CH}_{3}\right), 61.86\left(\mathrm{CH}_{3}\right), 61.89\left(\mathrm{CH}_{3}\right), 62.18$ $\left(\mathrm{CH}_{3}\right), 62.25\left(\mathrm{CH}_{3}\right), 64.71\left(\mathrm{CH}_{2}, \mathrm{C}^{\mathrm{I}}\right.$ or $\left.\mathrm{C}^{\mathrm{IV}}\right), 65.14\left(\mathrm{CH}_{2}, \mathrm{C}^{\mathrm{I}}\right.$ or $\left.\mathrm{C}^{\mathrm{IV}}\right), 66.65\left(\mathrm{CH}, \mathrm{C}^{\mathrm{I}}\right.$ or $\left.\mathrm{C}^{\mathrm{IV}}\right)$, $67.21\left(\mathrm{CH}, \mathrm{C}^{\mathrm{I}}\right.$ or $\left.\mathrm{C5}^{\mathrm{IV}}\right), 70.76\left(\mathrm{CH}_{2}\right), 70.85(\mathrm{CH}), 70.87(\mathrm{CH}), 70.92(\mathrm{CH}), 70.98\left(\mathrm{CH}_{2}\right), 71.10$ $\left(\mathrm{CH}_{2}\right), 71.74\left(\mathrm{CH}_{2}\right), 72.05\left(\mathrm{CH}_{2}\right), 77.64\left(\mathrm{CH}, \mathrm{C}^{\mathrm{I}}\right.$ or $\left.\mathrm{C}^{\mathrm{IV}}\right), 77.79\left(\mathrm{CH}, \mathrm{C} 4^{\mathrm{I}}\right.$ or $\left.\mathrm{C} 4^{\mathrm{IV}}\right), 78.00(\mathrm{CH})$, 
$79.14(\mathrm{CH}), 79.28(\mathrm{CH}), 79.95(\mathrm{CH}), 80.10(\mathrm{CH}), 80.48(\mathrm{CH}), 80.83(\mathrm{CH}), 80.98(\mathrm{CH}), 81.11$ $(\mathrm{CH}), 81.35(\mathrm{CH}), 81.50(\mathrm{CH}), 81.63(2 \times \mathrm{CH}), 81.74(\mathrm{CH}), 81.94(2 \times \mathrm{CH}), 82.78(\mathrm{CH}), 83.26$ $(\mathrm{CH}), 83.36(\mathrm{CH}), 96.92(\mathrm{CH}), 97.05(\mathrm{CH}), 97.78(\mathrm{CH}), 98.28(\mathrm{CH}), 99.08(\mathrm{CH}), 99.78\left(\mathrm{CH}, \mathrm{Cl}^{\mathrm{I}}\right.$ or $\left.\mathrm{C}^{\mathrm{IV}}\right), 100.59\left(\mathrm{CH}, \mathrm{C}^{\mathrm{I}}\right.$ or $\left.\mathrm{C} 1^{\mathrm{IV}}\right), 100.77\left(\mathrm{C}, \mathrm{C}^{\mathrm{I}}\right.$ or $\left.\mathrm{C} 5^{\mathrm{IV}}\right), 101.17\left(\mathrm{C}, \mathrm{C} 5^{\mathrm{I}}\right.$ or $\left.\mathrm{C} 5^{\mathrm{IV}}\right)$; $\mathrm{MS}\left(\mathrm{ESI}^{+}-\right.$ TOF): $m / z(\%) 1419\left[(\mathrm{M}+\mathrm{Na})^{+}, 100\right]$; HRMS (ESI $\left.{ }^{+}-\mathrm{TOF}\right): m / z[\mathrm{M}+\mathrm{Na}]^{+}$calcd for $\mathrm{C}_{61} \mathrm{H}_{104} \mathrm{NaO}_{35}$ 1419.6256; found 1419.6250. Anal. calcd for $\mathrm{C}_{61} \mathrm{H}_{104} \mathrm{O}_{35}$ : C, 52.43; H, 7.50. Found: C, 52.48; H, 7.34 .

Some spectroscopic data of the inseparable mixture of mono-trioxocane-mono-acetyl positional isomers could be obtained: colorless oil, ${ }^{1} \mathrm{H}$ NMR $\left(500 \mathrm{MHz}, \mathrm{CDCl}_{3}\right.$, complex spectrum, only clearly distinguished signals are reported): $\delta 2.062(\mathrm{~s}, 3 \mathrm{H}), 2.064$ (s, 3H), $4.15(\mathrm{dd}, J=2.5,2.5 \mathrm{~Hz}$, 1H), $4.17(\mathrm{dd}, J=2.4,2.4 \mathrm{~Hz}, 1 \mathrm{H}), 4.96(\mathrm{~d}, J=3.5 \mathrm{~Hz}, 1 \mathrm{H}), 5.01(\mathrm{~d}, J=3.5 \mathrm{~Hz}, 1 \mathrm{H}), 5.041(\mathrm{~d}, J$ $=3.5 \mathrm{~Hz}, 1 \mathrm{H}), 5.05(\mathrm{~d}, J=4.4 \mathrm{~Hz}, 2 \mathrm{H}), 5.06(\mathrm{~d}, J=3.2 \mathrm{~Hz}, 2 \mathrm{H}), 5.07(\mathrm{~d}, J=3.2 \mathrm{~Hz}, 1 \mathrm{H}), 5.10(\mathrm{~d}$, $J=3.5 \mathrm{~Hz}, 1 \mathrm{H}), 5.12(\mathrm{~d}, J=3.5 \mathrm{~Hz}, 1 \mathrm{H}), 5.14(\mathrm{~d}, J=2.2 \mathrm{~Hz}, 1 \mathrm{H}), 5.17(\mathrm{~d}, J=2.2 \mathrm{~Hz}, 1 \mathrm{H}), 5.227$ $(\mathrm{d}, J=3.4 \mathrm{~Hz}, 1 \mathrm{H}), 5.229(\mathrm{~d}, J=3.5 \mathrm{~Hz}, 1 \mathrm{H}) ;{ }^{13} \mathrm{C} \mathrm{NMR}\left(125.7 \mathrm{MHz}, \mathrm{CDCl}_{3}\right.$, complex spectrum, only clearly distinguished signals are reported): $\delta 22.02\left(\mathrm{CH}_{3}\right), 22.06\left(\mathrm{CH}_{3}\right), 60.31\left(\mathrm{CH}_{2}\right), 60.37$ $\left(\mathrm{CH}_{2}\right), 64.84\left(\mathrm{CH}_{2}\right), 64.91\left(\mathrm{CH}_{2}\right), 66.91(\mathrm{CH}), 67.07(\mathrm{CH}), 96.98(\mathrm{CH}), 97.01(\mathrm{CH}), 97.21(\mathrm{CH})$, $97.33(\mathrm{CH}), 97.46(\mathrm{CH}), 97.89(\mathrm{CH}), 99.12(\mathrm{CH}), 99.24(\mathrm{CH}), 99.62(\mathrm{CH}), 99.83(\mathrm{CH}), 100.17$ (CH), $100.24(\mathrm{CH}), 100.40(\mathrm{CH}), 101.05(\mathrm{C}), 101.45(\mathrm{C}), 101.93(\mathrm{CH}), 104.51(\mathrm{C}), 104.55(\mathrm{C})$, 170.14 (C); MS (ESI $\left.{ }^{+}-\mathrm{TOF}\right): m / z(\%) 1479\left[(\mathrm{M}+\mathrm{Na})^{+}, 100\right] ; \mathrm{HRMS}\left(\mathrm{ESI}^{+}-\mathrm{TOF}\right): m / z[\mathrm{M}+\mathrm{Na}]^{+}$ calcd for $\mathrm{C}_{63} \mathrm{H}_{108} \mathrm{NaO}_{37}$ 1479.6467; found 1479.6475.

Compound 13: colorless oil, $[\alpha]_{\mathrm{D}}+130.8\left(c\right.$ 0.75, $\left.\mathrm{CHCl}_{3}\right)$; IR (film): 2933, 1732, 1454, 1367, 1193, 1106, $1046 \mathrm{~cm}^{-1}$; ${ }^{1} \mathrm{H}$ NMR (500 MHz, $\left.\mathrm{CDCl}_{3}\right): \delta 2.04$ (s, 6H), $3.12(\mathrm{dd}, J=9.3,2.7 \mathrm{~Hz}, 1 \mathrm{H})$, $3.15(\mathrm{dd}, J=6.9,3.2 \mathrm{~Hz}, 1 \mathrm{H}), 3.16(\mathrm{dd}, J=7.6,3.5 \mathrm{~Hz}, 1 \mathrm{H}), 3.22(\mathrm{dd}, J=9.8,3.2 \mathrm{~Hz}, 1 \mathrm{H}), 3.26$ 
(dd, $J=9.8,3.5 \mathrm{~Hz}, 1 \mathrm{H}), 3.367$ (s, 6H), $3.370(\mathrm{~s}, 3 \mathrm{H}), 3.38(\mathrm{~s}, 3 \mathrm{H}), 3.39(\mathrm{~s}, 3 \mathrm{H}), 3.40(\mathrm{~s}, 6 \mathrm{H}), 3.458$ (s, 3H), 3.461 (s, 3H), 3.463 (s, 3H), 3.49 (s, 3H), 3.50 (s, 3H), 3.52 (s, 3H), $3.588(\mathrm{~s}, 3 \mathrm{H}), 3.591$ (s, 3H), 3.595 (s, 3H), 3.598 (s, 3H), 3.617 (s, 3H), 3.619 (s, 3H), 3.90 (d, J = $9.8 \mathrm{~Hz}, 1 \mathrm{H}, \mathrm{H} 6$ ), 3.95 (d, $J=9.8 \mathrm{~Hz}, 1 \mathrm{H}, \mathrm{H6}$ ), 4.00 (d, $J=9.8 \mathrm{~Hz}, 1 \mathrm{H}, \mathrm{H6}$ ), 4.03 (d, $J=9.8 \mathrm{~Hz}, 1 \mathrm{H}, \mathrm{H} 6), 4.05$ (br d, $\left.J=2.5 \mathrm{~Hz}, 1 \mathrm{H}, \mathrm{H} 4^{\mathrm{VII}}\right), 4.08\left(\mathrm{dd}, J=2.5,2.5 \mathrm{~Hz}, 1 \mathrm{H}, \mathrm{H} 3^{\mathrm{III}}\right), 4.16\left(\mathrm{dd}, J=2.5,2.5 \mathrm{~Hz}, 1 \mathrm{H}, \mathrm{H} 3^{\mathrm{VII}}\right)$, 4.33 (br dd, $J=11.0,7.3 \mathrm{~Hz}, 1 \mathrm{H}), 4.41$ (br dd, $J=11.5,5.8 \mathrm{~Hz}, 1 \mathrm{H}), 4.97$ (d, $J=2.8 \mathrm{~Hz}, 1 \mathrm{H}), 4.98$ (d, $J=3.2 \mathrm{~Hz}, 1 \mathrm{H}), 5.03(\mathrm{~d}, J=3.5 \mathrm{~Hz}, 1 \mathrm{H}), 5.04(\mathrm{~d}, J=3.2 \mathrm{~Hz}, 1 \mathrm{H}), 5.09(\mathrm{~d}, J=3.2 \mathrm{~Hz}, 1 \mathrm{H})$, $5.11\left(\mathrm{~d}, J=1.9 \mathrm{~Hz}, 1 \mathrm{H}, \mathrm{H} 1^{\mathrm{III}}\right), 5.14$ (d, $\left.J=1.9 \mathrm{~Hz}, 1 \mathrm{H}, \mathrm{H} 1^{\mathrm{VIII}}\right) ;{ }^{1} \mathrm{H} \mathrm{NMR}\left(500 \mathrm{MHz}, \mathrm{CDCl}_{3}, 1 \mathrm{D}-\right.$ TOCSY, irradiation at $\left.\mathrm{H} 1^{\mathrm{VII}}, 5.14 \mathrm{ppm}\right): \delta 3.40$ (br s, $\left.1 \mathrm{H}, \mathrm{H} 2^{\mathrm{VII}}\right), 4.05$ (br s, $\left.1 \mathrm{H}, \mathrm{H} 4^{\mathrm{VII}}\right), 4.17$ (dd, $\left.J=2.5,2.5 \mathrm{~Hz}, 1 \mathrm{H}, \mathrm{H} 3^{\mathrm{VII}}\right) ;{ }^{1} \mathrm{H} \mathrm{NMR}\left(500 \mathrm{MHz}, \mathrm{CDCl}_{3}, 1 \mathrm{D}-\mathrm{TOCSY}\right.$, irradiation at $\mathrm{H} 1^{\mathrm{III}}, 5.11$ ppm): $\delta 3.32$ (br s, $1 \mathrm{H}, \mathrm{H} 2^{\mathrm{III}}$ ), 4.02 (br s, $1 \mathrm{H}, \mathrm{H} 4^{\mathrm{III}}$ ), 4.09 (dd, $J=2.5,2.5 \mathrm{~Hz}, 1 \mathrm{H}, \mathrm{H} 3^{\mathrm{III}}$ ); ${ }^{1} \mathrm{H}$ NMR (500 MHz, $\left.\mathrm{C}_{6} \mathrm{D}_{6}, 18^{\circ} \mathrm{C}\right): \delta 1.74(\mathrm{~s}, 3 \mathrm{H}), 1.78$ (s, 3H), $3.16(\mathrm{~s}, 3 \mathrm{H}), 3.18(\mathrm{~s}, 3 \mathrm{H}), 3.19(\mathrm{~s}, 3 \mathrm{H}), 3.21$ (s, 3H), $3.26(\mathrm{~s}, 3 \mathrm{H}), 3.270(\mathrm{~s}, 3 \mathrm{H}), 3.274(\mathrm{~s}, 6 \mathrm{H}), 3.30(\mathrm{~s}, 9 \mathrm{H}), 3.40(\mathrm{~s}, 3 \mathrm{H}), 3.61(\mathrm{~s}, 3 \mathrm{H}), 3.62(\mathrm{~s}$, 3H), $3.66(\mathrm{~s}, 3 \mathrm{H}), 3.72(\mathrm{~s}, 3 \mathrm{H}), 3.822(\mathrm{~s}, 3 \mathrm{H}), 3.825(\mathrm{~s}, 3 \mathrm{H}), 3.85(\mathrm{~s}, 3 \mathrm{H}), 4.44(\mathrm{~d}, J=9.8 \mathrm{~Hz}, 1 \mathrm{H})$, $4.51(\mathrm{~d}, J=9.8 \mathrm{~Hz}, 1 \mathrm{H}), 5.08(\mathrm{~d}, J=3.2 \mathrm{~Hz}, 1 \mathrm{H}), 5.09(\mathrm{~d}, J=3.5 \mathrm{~Hz}, 1 \mathrm{H}), 5.15(\mathrm{~d}, J=2.5 \mathrm{~Hz}$, $1 \mathrm{H}), 5.18(\mathrm{~d}, J=3.8 \mathrm{~Hz}, 1 \mathrm{H}), 5.19(\mathrm{~d}, J=2.8 \mathrm{~Hz}, 1 \mathrm{H}), 5.59(\mathrm{~d}, J=1.3 \mathrm{~Hz}, 1 \mathrm{H}), 5.63(\mathrm{~d}, J=1.3$ $\mathrm{Hz}, 1 \mathrm{H}) ;{ }^{1} \mathrm{H}$ NMR $\left(500 \mathrm{MHz}, \mathrm{C}_{6} \mathrm{D}_{6}, 70{ }^{\circ} \mathrm{C}\right): \delta 1.79(\mathrm{~s}, 3 \mathrm{H}), 1.83(\mathrm{~s}, 3 \mathrm{H}), 3.21(\mathrm{~s}, 3 \mathrm{H}), 3.23(\mathrm{~s}, 3 \mathrm{H})$, 3.25 (s, 3H), 3.27 (s, 3H), 3.29 (s, 3H), 3.308 (s, 3H), 3.312 (s, 3H), $3.313(\mathrm{~s}, 3 \mathrm{H}), 3.319$ (s, 3H), $3.322(\mathrm{~s}, 3 \mathrm{H}), 3.34(\mathrm{~s}, 3 \mathrm{H}), 3.39(\mathrm{~s}, 3 \mathrm{H}), 3.60(\mathrm{~s}, 3 \mathrm{H}), 3.61(\mathrm{~s}, 3 \mathrm{H}), 3.699(\mathrm{~s}, 3 \mathrm{H}), 3.702(\mathrm{~s}, 3 \mathrm{H})$, $3.79(\mathrm{~s}, 3 \mathrm{H}), 3.80(\mathrm{~s}, 3 \mathrm{H}), 3.83(\mathrm{~s}, 3 \mathrm{H}), 4.10(\mathrm{dd}, J=11.2,3.9 \mathrm{~Hz}, 1 \mathrm{H}), 4.40(\mathrm{~d}, J=9.8 \mathrm{~Hz}, 1 \mathrm{H})$, $4.44(\mathrm{~d}, J=9.8 \mathrm{~Hz}, 1 \mathrm{H}), 5.05(\mathrm{~d}, J=3.2 \mathrm{~Hz}, 1 \mathrm{H}), 5.07(\mathrm{~d}, J=3.5 \mathrm{~Hz}, 1 \mathrm{H}), 5.11(\mathrm{~d}, J=2.5 \mathrm{~Hz}$, $1 \mathrm{H}), 5.13(\mathrm{~d}, J=3.5 \mathrm{~Hz}, 1 \mathrm{H}), 5.16(\mathrm{~d}, J=2.8 \mathrm{~Hz}, 1 \mathrm{H}), 5.56(\mathrm{~d}, J=1.6 \mathrm{~Hz}, 1 \mathrm{H}), 5.65(\mathrm{~d}, J=1.3$ $\mathrm{Hz}, 1 \mathrm{H}) ;{ }^{13} \mathrm{C} \mathrm{NMR}\left(125.7 \mathrm{MHz}, \mathrm{CDCl}_{3}\right): \delta 21.98\left(\mathrm{CH}_{3}\right), 22.03\left(\mathrm{CH}_{3}\right), 57.16\left(\mathrm{CH}_{3}\right), 57.41\left(\mathrm{CH}_{3}\right)$, 
$57.68\left(\mathrm{CH}_{3}\right), 57.75\left(\mathrm{CH}_{3}\right), 57.86\left(\mathrm{CH}_{3}\right), 58.84\left(\mathrm{CH}_{3}\right), 58.90\left(\mathrm{CH}_{3}\right), 58.99\left(4 \times \mathrm{CH}_{3}\right), 59.03\left(\mathrm{CH}_{3}\right)$, $59.34\left(\mathrm{CH}_{3}\right), 59.68\left(\mathrm{CH}_{3}\right), 59.91\left(\mathrm{CH}_{2}\right), 60.37\left(\mathrm{CH}_{2}\right), 61.02\left(\mathrm{CH}_{3}\right), 61.11\left(\mathrm{CH}_{3}\right), 61.55\left(\mathrm{CH}_{3}\right), 61.72$ $\left(\mathrm{CH}_{3}\right), 61.77\left(\mathrm{CH}_{3}\right), 70.44(\mathrm{CH}), 70.48\left(\mathrm{CH}_{2}\right), 70.60\left(\mathrm{CH}_{2}\right), 70.66\left(\mathrm{CH}_{2}\right), 70.86\left(\mathrm{CH}_{2}\right), 70.87\left(\mathrm{CH}_{2}\right)$, $71.08(\mathrm{CH}), 71.30(2 \times \mathrm{CH}), 71.59(\mathrm{CH}), 71.67(\mathrm{CH}), 72.16(\mathrm{CH}), 76.11\left(\mathrm{CH}, \mathrm{C} 3^{\mathrm{VII}}\right), 76.65(\mathrm{CH}$, $\left.\mathrm{C}^{\mathrm{VII}}\right), 76.86\left(\mathrm{CH}, \mathrm{C} 2^{\mathrm{III}}\right), 77.06\left(\mathrm{CH}, \mathrm{C}^{\mathrm{III}}\right), 77.99(\mathrm{CH}), 80.39(\mathrm{CH}), 81.55(\mathrm{CH}), 81.60(2 \times \mathrm{CH})$, $81.67(\mathrm{CH}), 82.02(\mathrm{CH}), 82.06(\mathrm{CH}), 82.15(\mathrm{CH}), 82.17(\mathrm{CH}), 82.20(\mathrm{CH}), 82.34(\mathrm{CH}), 82.68$ $(\mathrm{CH}), 82.84(\mathrm{CH}), 83.13(\mathrm{CH}), 97.70\left(\mathrm{CH}, \mathrm{Cl}^{\mathrm{III}}\right), 98.39\left(\mathrm{CH}, \mathrm{Cl}^{\mathrm{VII}}\right), 99.67(\mathrm{CH}), 99.70(\mathrm{CH})$, $99.82(\mathrm{CH}), 100.24(\mathrm{CH}), 101.71(\mathrm{CH}), 104.92\left(\mathrm{C}, \mathrm{C}^{\mathrm{III}}\right), 105.15\left(\mathrm{C}, \mathrm{C}^{\mathrm{VII}}\right), 170.08(\mathrm{C}), 170.14$ (C); MS (ESI $\left.{ }^{+}-\mathrm{TOF}\right): m / z(\%) 1539\left[(\mathrm{M}+\mathrm{Na})^{+}, 100\right] ; \mathrm{HRMS}\left(\mathrm{ESI}^{+}-\mathrm{TOF}\right): m / z[\mathrm{M}+\mathrm{Na}]^{+}$calcd for $\mathrm{C}_{65} \mathrm{H}_{112} \mathrm{NaO}_{39}$ 1539.6678; found 1539.6642. Anal. calcd for $\mathrm{C}_{65} \mathrm{H}_{112} \mathrm{O}_{39}$ : C, 51.44; $\mathrm{H}, 7.44$. Found: C, 51.49; H, 7.60.

Oxidative HAT of $2^{I-V I}, 3^{I-V I}, 6^{I I, I I, V, V I}$-hexadeca-O-methyl- $\alpha$-cyclomaltohexaose (14). Method A: A solution of alcohol $14^{25,29}(90 \mathrm{mg}, 0.075 \mathrm{mmol})$ in dry $\mathrm{CH}_{2} \mathrm{Cl}_{2}(3 \mathrm{~mL})$ containing DIB (53 mg, $0.165 \mathrm{mmol})$ and $\mathrm{I}_{2}(9.5 \mathrm{mg}, 0.038 \mathrm{mmol})$ was stirred under nitrogen at $26{ }^{\circ} \mathrm{C}$ for $1.5 \mathrm{~h}$ while irradiated with two $80 \mathrm{~W}$ tungsten-filament lamps. The reaction mixture was then directly loaded onto a silica gel (TLC Silica gel $60 \mathrm{~F}_{254}$, scraped from Merck Aluminum sheets) column chromatography (hexanes-acetone, $65: 35 \rightarrow 55: 45)$ to give cyclo- $5^{\mathrm{VI}}, 6^{\mathrm{I}}$-anhydro- $\left(5^{\mathrm{VI}} R\right)-(2,3,6$-tri- $O$ methyl- $\alpha$-D-xylo-hexos-5-ulopyranosyl)-( $1 \rightarrow 4)$-2,3-di- $O$-methyl- $\alpha$-D-glucopyranosyl-( $1 \rightarrow 4)$ 2,3,6-tri- $O$-methyl- $\alpha$-D-glucopyranosyl-( $(1 \rightarrow 4)-5^{\mathrm{III}}, 6^{\mathrm{IV}}$-anhydro-( $\left.5^{\mathrm{III}} R\right)-(2,3,6$-tri- $O$-methyl- $\alpha$-D$x y l o$-hexos-5-ulopyranosyl)-(1 $\rightarrow 4)$-2,3-di- $O$-methyl- $\alpha$-D-glucopyranosyl-( $1 \rightarrow 4)-2,3,6$-tri- $O$ methyl- $\alpha$-D-glucopyranosyl (15) (25.6 mg, $0.021 \mathrm{mmol}, 29 \%)$ and cyclo- $5^{\mathrm{VI}}, 6^{\mathrm{I}}$-anhydro- $\left(5^{\mathrm{VI}} R\right)-$ (2,3,6-tri-O-methyl- $\alpha$-D-xylo-hexos-5-ulopyranosyl)-(1 $\rightarrow 4)$-2,3-di-O-methyl- $\alpha$-Dglucopyranosyl-[( $1 \rightarrow 4)$-2,3,6-tri- $O$-methyl- $\alpha$-D-glucopyranosyl] 2 -(1 $\rightarrow 4)-2,3$-di- $O$-methyl- $\alpha$-D- 
glucopyranosyl-(1 $\rightarrow 4$ )-2,3,6-tri- $O$-methyl- $\alpha$-D-glucopyranosyl (16) $(17.5 \mathrm{mg}, 0.015 \mathrm{mmol}, 19 \%)$. Compound 15: Crystalline solid m.p. $250-252{ }^{\circ} \mathrm{C}$ (from $n$-hexane-EtOAc); $[\alpha]_{\mathrm{D}}+133.0(c$ 1.176, $\mathrm{CHCl}_{3}$ ); IR (film): 2929, 1454, 1365, 1139, 1109, $1063 \mathrm{~cm}^{-1}$; ${ }^{1} \mathrm{H}$ NMR (500 MHz, $\mathrm{CDCl}_{3}$ ): $\delta 3.14$ (dd, $J=9.1,3.5 \mathrm{~Hz}, 2 \mathrm{H}), 3.20(\mathrm{dd}, J=9.5,3.5 \mathrm{~Hz}, 2 \mathrm{H}), 3.25(\mathrm{dd}, J=9.8,3.5 \mathrm{~Hz}, 2 \mathrm{H}), 3.368(\mathrm{~s}$, 6H), 3.369 (s, 6H), 3.47 (s, 6H), 3.48 (s, 6H), 3.50 (s, 6H), 3.53 (dd, $J=9.5,9.5 \mathrm{~Hz}, 2 \mathrm{H}), 3.61$ (s, $12 \mathrm{H}), 3.69(\mathrm{dd}, J=9.3,9.3 \mathrm{~Hz}, 2 \mathrm{H}), 3.74(\mathrm{~s}, 6 \mathrm{H}), 3.77(\mathrm{dd}, J=9.5,9.5 \mathrm{~Hz}, 2 \mathrm{H}), 3.93(\mathrm{~d}, J=9.5$ $\mathrm{Hz}, 2 \mathrm{H}), 3.98$ (d, $J=9.8 \mathrm{~Hz}, 2 \mathrm{H}), 4.99(\mathrm{~d}, J=3.8 \mathrm{~Hz}, 2 \mathrm{H}), 5.02(\mathrm{~d}, J=3.5 \mathrm{~Hz}, 2 \mathrm{H}), 5.19$ (d, $J=$ $3.2 \mathrm{~Hz}, 2 \mathrm{H}) ;{ }^{13} \mathrm{C} \mathrm{NMR}\left(125.7 \mathrm{MHz}, \mathrm{CDCl}_{3}\right): \delta 57.62\left(2 \times \mathrm{CH}_{3}\right), 57.80\left(2 \times \mathrm{CH}_{3}\right), 58.13\left(2 \times \mathrm{CH}_{3}\right)$, $58.98\left(2 \times \mathrm{CH}_{3}\right), 59.14\left(2 \times \mathrm{CH}_{3}\right), 61.28\left(2 \times \mathrm{CH}_{3}\right), 62.06\left(2 \times \mathrm{CH}_{3}\right), 62.14\left(2 \times \mathrm{CH}_{3}\right), 64.01(2 \times$ $\left.\mathrm{CH}_{2}\right), 66.49(2 \times \mathrm{CH}), 70.69\left(2 \times \mathrm{CH}_{2}\right), 70.91(2 \times \mathrm{CH}), 72.25\left(2 \times \mathrm{CH}_{2}\right), 78.32(2 \times \mathrm{CH}), 80.38$ $(2 \times \mathrm{CH}), 80.67(2 \times \mathrm{CH}), 80.83(2 \times \mathrm{CH}), 81.24(2 \times \mathrm{CH}), 81.42(2 \times \mathrm{CH}), 81.73(2 \times \mathrm{CH}), 82.07$ $(2 \times \mathrm{CH}), 82.21(2 \times \mathrm{CH}), 97.28(2 \times \mathrm{CH}), 98.63(2 \times \mathrm{CH}), 99.13(2 \times \mathrm{CH}), 100.37(2 \times \mathrm{C}) ; \mathrm{MS}$ (ESI ${ }^{+}$-TOF): $m / z(\%) 1215\left[(\mathrm{M}+\mathrm{Na})^{+}, 100\right]$; HRMS $\left(\mathrm{ESI}^{+}-\mathrm{TOF}\right): m / z[\mathrm{M}+\mathrm{Na}]^{+}$calcd for $\mathrm{C}_{52} \mathrm{H}_{88} \mathrm{NaO}_{30}$ 1215.5258; found 1215.5253. Anal. calcd for $\mathrm{C}_{52} \mathrm{H}_{88} \mathrm{O}_{30}$ : C, 52.34; H, 7.43. Found: C, 52.41; H, 7.26. Compound 16: colorless oil, $[\alpha]_{\mathrm{D}}+108.2\left(c\right.$ 1.48, $\left.\mathrm{CHCl}_{3}\right)$; IR (film): 3475, 2929 , 1454, 1365, 1141, 1107, $1046 \mathrm{~cm}^{-1} ;{ }^{1} \mathrm{H}$ NMR (500 MHz, $\left.\mathrm{CDCl}_{3}\right): \delta 3.12-3.21(\mathrm{~m}, 5 \mathrm{H}), 3.25(\mathrm{dd}$, $J=9.8,3.2 \mathrm{~Hz}, 1 \mathrm{H}), 3.36(\mathrm{~s}, 3 \mathrm{H}), 3.37$ (s, 3H), $3.379(\mathrm{~s}, 3 \mathrm{H}), 3.380(\mathrm{~s}, 3 \mathrm{H}), 3.47$ (s, 3H), $3.47(\mathrm{~s}$, $6 \mathrm{H}), 3.48(\mathrm{~s}, 3 \mathrm{H}), 3.50(\mathrm{~s}, 3 \mathrm{H}), 3.53(\mathrm{~s}, 3 \mathrm{H}), 3.60(\mathrm{~s}, 3 \mathrm{H}), 3.61(\mathrm{~s}, 3 \mathrm{H}), 3.618(\mathrm{~s}, 3 \mathrm{H}), 3.620(\mathrm{~s}, 3 \mathrm{H})$, $3.71(\mathrm{~s}, 3 \mathrm{H}), 3.73(\mathrm{~s}, 3 \mathrm{H}), 4.96(\mathrm{~d}, J=3.5 \mathrm{~Hz}, 1 \mathrm{H}), 4.97(\mathrm{~d}, J=3.5 \mathrm{~Hz}, 1 \mathrm{H}), 5.03(\mathrm{~d}, J=3.5 \mathrm{~Hz}$, $1 \mathrm{H}), 5.04(\mathrm{~d}, J=3.5 \mathrm{~Hz}, 1 \mathrm{H}), 5.16(\mathrm{~d}, J=3.8 \mathrm{~Hz}, 1 \mathrm{H}), 5.19(\mathrm{~d}, J=3.5 \mathrm{~Hz}, 1 \mathrm{H}) ;{ }^{13} \mathrm{C}$ NMR $(125.7$ $\left.\mathrm{MHz}, \mathrm{CDCl}_{3}\right): \delta 57.65\left(\mathrm{CH}_{3}\right), 57.83\left(\mathrm{CH}_{3}\right), 57.85\left(\mathrm{CH}_{3}\right), 57.86\left(\mathrm{CH}_{3}\right), 58.39\left(\mathrm{CH}_{3}\right), 58.72\left(\mathrm{CH}_{3}\right)$, $59.07\left(3 \times \mathrm{CH}_{3}\right), 59.09\left(\mathrm{CH}_{3}\right), 61.25\left(2 \times \mathrm{CH}_{3}\right), 61.64\left(\mathrm{CH}_{3}\right), 61.89\left(\mathrm{CH}_{3}\right), 61.95\left(\mathrm{CH}_{2}\right), 61.98$ $\left(\mathrm{CH}_{3}\right), 62.25\left(\mathrm{CH}_{3}\right), 64.05\left(\mathrm{CH}_{2}\right), 66.40(\mathrm{CH}), 70.95\left(\mathrm{CH}_{2}\right), 71.03(\mathrm{CH}), 71.14(\mathrm{CH}), 71.39\left(\mathrm{CH}_{2}\right)$, 
$71.81(\mathrm{CH}), 72.03\left(\mathrm{CH}_{2}\right), 72.07\left(\mathrm{CH}_{2}\right), 72.10(\mathrm{CH}), 78.18(\mathrm{CH}), 80.09(\mathrm{CH}), 80.37(\mathrm{CH}), 80.46$ $(\mathrm{CH}), 80.75(\mathrm{CH}), 80.83(\mathrm{CH}), 80.86(\mathrm{CH}), 81.26(\mathrm{CH}), 81.33(\mathrm{CH}), 81.50(\mathrm{CH}), 81.61(\mathrm{CH})$, $81.65(\mathrm{CH}), 81.79(\mathrm{CH}), 81.96(\mathrm{CH}), 82.14(\mathrm{CH}), 82.45(\mathrm{CH}), 82.59(\mathrm{CH}), 82.78(\mathrm{CH}), 97.30$ (CH), $97.97(\mathrm{CH}), 98.34(\mathrm{CH}), 99.39(2 \times \mathrm{CH}), 99.52(\mathrm{CH}), 100.79(\mathrm{C})$; MS (ESI $\left.{ }^{+} \mathrm{TOF}\right): \mathrm{m} / z(\%)$ $1217\left[(\mathrm{M}+\mathrm{Na})^{+}, 100\right] ;$ HRMS (ESI ${ }^{+}$TOF): $m / z[\mathrm{M}+\mathrm{Na}]^{+}$calcd for $\mathrm{C}_{52} \mathrm{H}_{90} \mathrm{NaO}_{30}$ 1217.5415; found 1217.5419. Anal. calcd for $\mathrm{C}_{52} \mathrm{H}_{90} \mathrm{O}_{30}: \mathrm{C}, 52.25 ; \mathrm{H}, 7.59$. Found: $\mathrm{C}, 52.33 ; \mathrm{H}, 7.37$.

Method B: A solution of alcohol $14(45 \mathrm{mg}, 0.038 \mathrm{mmol})$ in dry $\mathrm{CH}_{2} \mathrm{Cl}_{2}(1.5 \mathrm{~mL})$ containing DIB (30 mg, $0.095 \mathrm{mmol})$ and $\mathrm{I}_{2}(14.5 \mathrm{mg}, 0.057 \mathrm{mmol})$ was stirred under nitrogen at $28{ }^{\circ} \mathrm{C}$ for $1.5 \mathrm{~h}$ while irradiated with two $80 \mathrm{~W}$ tungsten-filament lamps. The reaction mixture was then directly loaded onto a silica gel (TLC Silica gel $60 \mathrm{~F}_{254}$, scraped from Merck Aluminum sheets) column chromatography (hexanes-acetone, $65: 35 \rightarrow 55: 45)$ to give $15(13 \mathrm{mg}, 0.011 \mathrm{mmol}, 29 \%)$ and 16 (7.2 mg, $0.006 \mathrm{mmol}, 16 \%)$.

Method C: A solution of alcohol $14(47 \mathrm{mg}, 0.039 \mathrm{mmol})$ in dry $\mathrm{CH}_{2} \mathrm{Cl}_{2}(1.6 \mathrm{~mL})$ containing DIB $(37.7 \mathrm{mg}, 0.117 \mathrm{mmol})$ and $\mathrm{I}_{2}(16.8 \mathrm{mg}, 0.066 \mathrm{mmol})$ was stirred under nitrogen at $28{ }^{\circ} \mathrm{C}$ for $1.5 \mathrm{~h}$ while irradiated with two $80 \mathrm{~W}$ tungsten-filament lamps. The reaction mixture was then directly loaded onto a silica gel (TLC Silica gel $60 \mathrm{~F}_{254}$, scraped from Merck Aluminum sheets) column chromatography (hexanes-acetone, 65:35) to give 15 (15.9 mg, $0.013 \mathrm{mmol}, 34 \%)$.

Oxidative HAT of mono-trioxocane alcohol (16). A solution of alcohol 16 (12 mg, $0.01 \mathrm{mmol})$ in dry $\mathrm{CH}_{2} \mathrm{Cl}_{2}(0.45 \mathrm{~mL})$ containing DIB $(7 \mathrm{mg}, 0.022 \mathrm{mmol})$ and $\mathrm{I}_{2}(1.3 \mathrm{mg}, 0.005 \mathrm{mmol})$ was stirred under nitrogen at $28^{\circ} \mathrm{C}$ for $2 \mathrm{~h}$ while irradiated with two $80 \mathrm{~W}$ tungsten-filament lamps. The reaction mixture was then directly loaded onto a silica gel (TLC Silica gel $60 \mathrm{~F}_{254}$, scraped from Merck Aluminum sheets) column chromatography (hexanes-acetone, 65:35) to give bis(trioxocane) $\mathbf{1 5}$ (6 $\mathrm{mg}, 0.005 \mathrm{mmol}, 50 \%$ ). 
Oxidative HAT of $2^{I-V I I}, 3^{I-V I I}, 6^{I I I V-V I I}$-nonadeca-O-methyl- $\beta$-cyclomaltoheptaose (17). A solution of alcohol $17^{17 \mathrm{a}}(119 \mathrm{mg}, 0.085 \mathrm{mmol})$ in dry $\mathrm{CH}_{2} \mathrm{Cl}_{2}(3.5 \mathrm{~mL})$ containing DIB $(60.2 \mathrm{mg}, 0.187$ $\mathrm{mmol})$ and $\mathrm{I}_{2}(28 \mathrm{mg}, 0.110 \mathrm{mmol})$ was stirred under nitrogen at $30{ }^{\circ} \mathrm{C}$ for $2.5 \mathrm{~h}$ while irradiated with two $80 \mathrm{~W}$ tungsten-filament lamps. The reaction mixture was then directly loaded onto a silica gel (TLC Silica gel $60 \mathrm{~F}_{254}$, scraped from Merck Aluminum sheets) column chromatography $\left(\mathrm{CHCl}_{3}-\right.$ $\mathrm{MeOH}, 99: 1 \rightarrow 98: 2)$ to give the acetyl derivative $\mathbf{1 8}$ as an inseparable mixture of positional isomers (26.1 mg, $0.018 \mathrm{mmol}, 21 \%, 70: 30)$, cyclo-(5R)-5 ${ }^{\mathrm{VII}}$ - $O$-acetyl-2,3,6-tri- $O$-methyl- $\alpha$-D-xylo-hexos5-ulopyranosyl-( $(1 \rightarrow 4)$-2,3-di-O-methyl- $\alpha$-D-glucopyranosyl-( $(1 \rightarrow 4)-(5 R)-5^{\mathrm{II}}$ - $O$-acetyl-2,3,6-tri$O$-methyl- $\alpha$-D-xylo-hexos-5-ulopyranosyl-( $1 \rightarrow 4)$-2,3-di-O-methyl- $\alpha$-D-glucopyranosyl-[( $1 \rightarrow 4)$ 2,3,6-tri- $O$-methyl- $\alpha$-D-glucopyranosyl] 3 (19) $(19.1 \mathrm{mg}, 0.013 \mathrm{mmol}, 15 \%)$, cyclo-(5R)-5 ${ }^{\mathrm{VII}}-O$ acetyl-2,3,6-tri- $O$-methyl- $\alpha$-D-xylo-hexos-5-ulopyranosyl-(1 $\rightarrow 4)$-2,3-di- $O$-methyl- $\alpha$-Dglucopyranosyl-( $(1 \rightarrow 4)-5^{\mathrm{II}}, 6^{\mathrm{III}}$-anhydro-( $\left(5^{\mathrm{II}} R\right)-(2,3,6$-tri- $O$-methyl- $\alpha$-D-xylo-hexos-5-

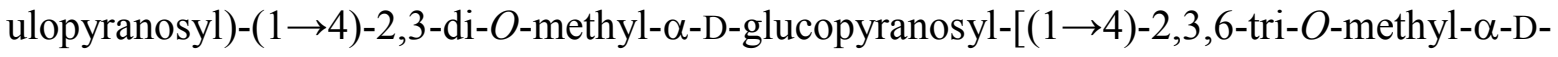
glucopyranosyl] 3 (20) (24.3 mg, $0.018 \mathrm{mmol}, 20 \%)$, cyclo-5 ${ }^{\mathrm{VII}}, 6^{\mathrm{I}}$-anhydro-(5 $\left.5^{\mathrm{VII}} R\right)-(2,3,6$-tri- $O$ methyl- $\alpha$-D-xylo-hexos-5-ulopyranosyl)-(1 $\rightarrow 4)$-2,3-di- $O$-methyl- $\alpha$-D-glucopyranosyl-(1 $\rightarrow 4)$ $(5 R)-5^{\text {II }} O-O$-acetyl-2,3,6-tri- $O$-methyl- $\alpha$-D-xylo-hexos-5-ulopyranosyl-(1 $\left.\rightarrow 4\right)$-2,3-di-O $O$-methyl- $\alpha$ D-glucopyranosyl-[(1 $\rightarrow 4)$-2,3,6-tri- $O$-methyl- $\alpha$-D-glucopyranosyl] 3 (21) (7 mg, 0.005 mmol, 6\%), cyclo-5 ${ }^{\mathrm{VII}}, 6^{\mathrm{I}}$-anhydro-( $\left.5^{\mathrm{VII}} R\right)-(2,3,6$-tri- $O$-methyl- $\alpha$-D-xylo-hexos-5-ulopyranosyl)-(1 $\rightarrow 4)-2,3$-di$O$-methyl- $\alpha$-D-glucopyranosyl-( $1 \rightarrow 4)-\left(1^{\mathrm{II}} R\right)-4^{\mathrm{III}}, 6^{\mathrm{III}}-O-(2,3$-di- $O$-methyl-Dglucopyranosylidene)-2,3-di- $O$-methyl- $\alpha$-D-glucopyranosyl-[(1 $\rightarrow 4)-2,3,6$-tri- $O$-methyl- $\alpha$-Dglucopyranosyl] 3 (22) (6.9 mg, $0.005 \mathrm{mmol}, 6 \%$ ). Compound 18: colorless oil, IR (film): 3516, $2933,1732,1455,1368,1105,1036 \mathrm{~cm}^{-1} ;{ }^{1} \mathrm{H}$ NMR $\left(500 \mathrm{MHz}, \mathrm{CDCl}_{3}\right)$ (only the major isomer is shown): $\delta 2.06(\mathrm{~s}, 3 \mathrm{H}), 3.18(\mathrm{~m}, 6 \mathrm{H}), 3.30(\mathrm{dd}, J=9.8,9.8 \mathrm{~Hz}, 1 \mathrm{H}), 3.36(\mathrm{~s}, 3 \mathrm{H}), 3.367(\mathrm{~s}, 3 \mathrm{H})$, 
$3.373(\mathrm{~s}, 3 \mathrm{H}), 3.39$ (s, 3H), 3.40 (s, 3H), 3.43 (s, 3H), 3.45 (s, 3H), $3.482(\mathrm{~s}, 3 \mathrm{H}), 3.483(\mathrm{~s}, 3 \mathrm{H})$, $3.49(\mathrm{~s}, 3 \mathrm{H}), 3.50(\mathrm{~s}, 3 \mathrm{H}), 3.54(\mathrm{~s}, 3 \mathrm{H}), 3.59(\mathrm{~s}, 3 \mathrm{H}), 3.61(\mathrm{~s}, 3 \mathrm{H}), 3.617(\mathrm{~s}, 3 \mathrm{H}), 3.621(\mathrm{~s}, 3 \mathrm{H})$, 3.627 (s, 3H), 3.633 (s, 3H), $3.64(\mathrm{~s}, 3 \mathrm{H}), 3.94$ (d, $J=9.8 \mathrm{~Hz}, 1 \mathrm{H}), 4.02(\mathrm{~d}, J=9.8 \mathrm{~Hz}, 1 \mathrm{H}), 4.05$ (d, $J=1.9 \mathrm{~Hz}, 1 \mathrm{H}), 4.18(\mathrm{dd}, J=2.4,2.4 \mathrm{~Hz}, 1 \mathrm{H}), 4.30(\mathrm{dd}, J=11.2,5.8 \mathrm{~Hz}, 1 \mathrm{H}), 5.01(\mathrm{~d}, J=3.2$ Hz, 1H), 5.037 (d, $J=3.4 \mathrm{~Hz}, 1 \mathrm{H}), 5.044(\mathrm{~d}, J=3.8 \mathrm{~Hz}, 1 \mathrm{H}), 5.079$ (d, $J=4.1 \mathrm{~Hz}, 1 \mathrm{H}), 5.087$ (d, $J=4.1 \mathrm{~Hz}, 1 \mathrm{H}), 5.12(\mathrm{~d}, J=1.9 \mathrm{~Hz}, 1 \mathrm{H}), 5.16(\mathrm{~d}, J=3.8 \mathrm{~Hz}, 1 \mathrm{H}) ;{ }^{1} \mathrm{H} \mathrm{NMR}\left(500 \mathrm{MHz}, \mathrm{CDCl}_{3}\right.$, 1D-ROESY, irradiation at $2.06 \mathrm{ppm}): \delta 5.12$ (d, $\left.J=1.9 \mathrm{~Hz}, 1 \mathrm{H}, \mathrm{H} 1{ }^{\mathrm{VII}}\right) ;{ }^{13} \mathrm{C}$ NMR $(125.7 \mathrm{MHz}$, $\left.\mathrm{CDCl}_{3}\right)$ (only the major isomer is shown): $\delta 22.10\left(\mathrm{CH}_{3}\right), 57.21\left(\mathrm{CH}_{3}\right), 57.50\left(\mathrm{CH}_{3}\right), 58.24\left(\mathrm{CH}_{3}\right)$, $58.53\left(\mathrm{CH}_{3}\right), 58.57\left(\mathrm{CH}_{3}\right), 58.88\left(2 \times \mathrm{CH}_{3}\right), 58.91\left(\mathrm{CH}_{3}\right), 58.95\left(\mathrm{CH}_{3}\right), 58.97\left(2 \times \mathrm{CH}_{3}\right), 59.01$ $\left(\mathrm{CH}_{3}\right), 59.20\left(\mathrm{CH}_{3}\right), 60.20\left(\mathrm{CH}_{2}\right), 61.22\left(\mathrm{CH}_{3}\right), 61.23\left(\mathrm{CH}_{3}\right), 61.37\left(\mathrm{CH}_{3}\right), 61.41\left(\mathrm{CH}_{3}\right), 61.50$ $\left(\mathrm{CH}_{3}\right), 61.67\left(\mathrm{CH}_{3}\right), 61.82\left(\mathrm{CH}_{2}\right), 70.33\left(\mathrm{CH}_{2}\right), 70.33(\mathrm{CH}), 71.02(2 \times \mathrm{CH}), 71.02\left(\mathrm{CH}_{2}\right), 71.13(2$ $\left.\times \mathrm{CH}_{2}\right), 71.14(2 \times \mathrm{CH}), 71.27\left(\mathrm{CH}_{2}\right), 71.42(\mathrm{CH}), 72.21(\mathrm{CH}), 75.42(\mathrm{CH}), 76.81(\mathrm{CH}), 79.73$ $(\mathrm{CH}), 80.17(\mathrm{CH}), 80.31(\mathrm{CH}), 81.13(2 \times \mathrm{CH}), 81.52(\mathrm{CH}), 81.62(\mathrm{CH}), 81.66(2 \times \mathrm{CH}), 81.73(2$ $\times \mathrm{CH}), 81.90(\mathrm{CH}), 82.02(\mathrm{CH}), 82.30(\mathrm{CH}), 82.33(\mathrm{CH}), 82.44(2 \times \mathrm{CH}), 83.67(\mathrm{CH}), 97.69(\mathrm{CH})$, $99.10(\mathrm{CH}), 99.35(\mathrm{CH}), 99.37(\mathrm{CH}), 99.41(\mathrm{CH}), 99.62(\mathrm{CH}), 100.10(\mathrm{CH}), 104.99(\mathrm{C}), 170.18$ (C); MS (ESI $\left.{ }^{+}-\mathrm{TOF}\right): m / z(\%) 1481\left[(\mathrm{M}+\mathrm{Na})^{+}, 100\right] ; \mathrm{HRMS}\left(\mathrm{ESI}^{+}-\mathrm{TOF}\right): m / z[\mathrm{M}+\mathrm{Na}]^{+}$calcd for $\mathrm{C}_{63} \mathrm{H}_{110} \mathrm{NaO}_{37}$ 1481.6624; found 1481.6647. Anal. calcd for $\mathrm{C}_{63} \mathrm{H}_{110} \mathrm{O}_{37}$ : C, 51.84; H, 7.60. Found: C, 51.60; H, 7.45. Compound 19: colorless oil, $[\alpha]_{\mathrm{D}}+118.9\left(c\right.$ 1.34, $\left.\mathrm{CHCl}_{3}\right)$; IR (film): 3521, 2928, 1732, 1455, 1368, 1195, 1105, $1041 \mathrm{~cm}^{-1} ;{ }^{1} \mathrm{H}$ NMR (500 MHz, $\left.\mathrm{CDCl}_{3}\right): \delta 2.04$ (s, 3H), 2.05 (s, 3H), $3.16(\mathrm{dd}, J=9.8,3.8 \mathrm{~Hz}, 2 \mathrm{H}), 3.17(\mathrm{dd}, J=9.5,3.2 \mathrm{~Hz}, 1 \mathrm{H}), 3.21(\mathrm{dd}, J=9.8,3.5 \mathrm{~Hz}$, $1 \mathrm{H}), 3.25(\mathrm{dd}, J=9.9,3.3 \mathrm{~Hz}, 1 \mathrm{H}), 3.30(\mathrm{dd}, J=9.8,9.8 \mathrm{~Hz}, 1 \mathrm{H}), 3.32(\mathrm{dd}, J=2.2,2.2 \mathrm{~Hz}, 1 \mathrm{H}$, H2), $3.36(\mathrm{~s}, 3 \mathrm{H}), 3.37$ (s, 3H), $3.38(\mathrm{~s}, 3 \mathrm{H}), 3.39$ (s, 3H), $3.40(\mathrm{~s}, 3 \mathrm{H}), 3.41(\mathrm{~s}, 3 \mathrm{H}), 3.42(\mathrm{~s}, 3 \mathrm{H})$, $3.46(\mathrm{~s}, 3 \mathrm{H}), 3.47(\mathrm{~s}, 3 \mathrm{H}), 3.479(\mathrm{~s}, 3 \mathrm{H}), 3.482(\mathrm{~s}, 3 \mathrm{H}), 3.53(\mathrm{~s}, 3 \mathrm{H}), 3.54(\mathrm{~s}, 3 \mathrm{H}), 3.586(\mathrm{~s}, 3 \mathrm{H})$, 
$3.593(\mathrm{~s}, 3 \mathrm{H}), 3.60(\mathrm{~s}, 3 \mathrm{H}), 3.61$ (s, 3H), 3.62 (s, 3H), 3.64 (s, 3H), 4.01 (br d, J=3.2 Hz, 1H, H4), $4.04(\mathrm{dd}, J=2.5,2.5 \mathrm{~Hz}, 1 \mathrm{H}, \mathrm{H} 3), 4.06$ (d, $J=2.2 \mathrm{~Hz}, 1 \mathrm{H}, \mathrm{H} 4), 4.18$ (dd, $J=2.5,2.5 \mathrm{~Hz}, 1 \mathrm{H}, \mathrm{H} 3$ ), $4.99(\mathrm{~d}, J=3.2 \mathrm{~Hz}, 1 \mathrm{H}), 5.00(\mathrm{~d}, J=4.1 \mathrm{~Hz}, 1 \mathrm{H}), 5.07$ (d, $J=2.2 \mathrm{~Hz}, 1 \mathrm{H}, \mathrm{H} 1), 5.079$ (d, $J=3.5$ $\mathrm{Hz}, 1 \mathrm{H}), 5.082(\mathrm{~d}, J=3.5 \mathrm{~Hz}, 1 \mathrm{H}), 5.10(\mathrm{~d}, J=3.5 \mathrm{~Hz}, 1 \mathrm{H}), 5.12(\mathrm{~d}, J=1.9 \mathrm{~Hz}, 1 \mathrm{H}, \mathrm{H} 1) ;{ }^{1} \mathrm{H}$ NMR (500 MHz, $\left.\mathrm{C}_{6} \mathrm{D}_{6}\right): \delta 1.68(\mathrm{~s}, 3 \mathrm{H}), 1.84(\mathrm{~s}, 3 \mathrm{H}), 3.01(\mathrm{dd}, J=9.1,2.8 \mathrm{~Hz}, 1 \mathrm{H}), 3.10(\mathrm{dd}, J=9.5,3.5$ Hz, 1H), 3.14 (s, 3H), $3.21(\mathrm{~s}, 3 \mathrm{H}), 3.22(\mathrm{~s}, 3 \mathrm{H}), 3.22(\mathrm{~s}, 3 \mathrm{H}), 3.24(\mathrm{~s}, 3 \mathrm{H}), 3.28(\mathrm{~s}, 3 \mathrm{H}), 3.31(\mathrm{~s}$, 3H), $3.35(\mathrm{~s}, 3 \mathrm{H}), 3.36(\mathrm{~s}, 3 \mathrm{H}), 3.40(\mathrm{~s}, 3 \mathrm{H}), 3.41(\mathrm{~s}, 6 \mathrm{H}), 3.42(\mathrm{~s}, 3 \mathrm{H}), 3.48(\mathrm{~s}, 3 \mathrm{H}), 3.65(\mathrm{~s}, 3 \mathrm{H})$, $3.66(\mathrm{~s}, 6 \mathrm{H}), 3.69$ (s, 3H), 3.79 (s, 3H), 5.00 (d, $J=2.8 \mathrm{~Hz}, 1 \mathrm{H}), 5.14$ (d, $J=3.2 \mathrm{~Hz}, 1 \mathrm{H}), 5.18$ (d, $J=3.2 \mathrm{~Hz}, 1 \mathrm{H}), 5.20(\mathrm{~d}, J=3.5 \mathrm{~Hz}, 1 \mathrm{H}), 5.32(\mathrm{~d}, J=3.8 \mathrm{~Hz}, 1 \mathrm{H}), 5.48(\mathrm{~d}, J=2.5 \mathrm{~Hz}, 1 \mathrm{H}), 5.53$ (d, $J=2.2 \mathrm{~Hz}, 1 \mathrm{H}) ;{ }^{1} \mathrm{H}$ NMR (500 MHz, $\mathrm{CDCl}_{3}, 1 \mathrm{D}-\mathrm{TOCSY}$, irradiation at $\mathrm{H} 1^{\mathrm{II}}$ or VII, $5.12 \mathrm{ppm}$ ): $\delta 3.42($ br s, $1 \mathrm{H}, \mathrm{H} 2), 4.05$ (br s, 1H, H4), 4.22 (br s, $1 \mathrm{H}, \mathrm{H} 3$ ); ${ }^{1} \mathrm{H}$ NMR $\left(500 \mathrm{MHz}, \mathrm{CDCl}_{3}, 1 \mathrm{D}-\right.$ TOCSY, irradiation at H1 ${ }^{\mathrm{VII}}$ or II, $5.07 \mathrm{ppm}$ ): $\delta 3.36$ (br s, $\left.1 \mathrm{H}, \mathrm{H} 2\right), 4.08$ (dd, $J=2.5,2.5 \mathrm{~Hz}, 1 \mathrm{H}$, H3), 4.09 (br s, $1 \mathrm{H}, \mathrm{H} 4) ;{ }^{1} \mathrm{H}$ NMR (500 MHz, $\left.\mathrm{CDCl}_{3}, 2 \mathrm{D}-\mathrm{ROESY}\right)$ : both acetates show NOE interactions with their respective anomeric hydrogens: $\mathrm{H} 1{ }^{\mathrm{VII}}$ and $\mathrm{H} 1{ }^{\mathrm{II}}$; ${ }^{13} \mathrm{C}$ NMR $(125.7 \mathrm{MHz}$, $\left.\mathrm{CDCl}_{3}\right): \delta 22.05\left(\mathrm{CH}_{3}\right), 22.08\left(\mathrm{CH}_{3}\right), 57.33\left(\mathrm{CH}_{3}\right), 57.37\left(\mathrm{CH}_{3}\right), 57.58\left(\mathrm{CH}_{3}\right), 58.20\left(\mathrm{CH}_{3}\right), 58.38$ $\left(\mathrm{CH}_{3}\right), 58.71\left(\mathrm{CH}_{3}\right), 58.93\left(\mathrm{CH}_{3}\right), 58.96\left(5 \times \mathrm{CH}_{3}\right), 59.36\left(\mathrm{CH}_{3}\right), 59.94\left(\mathrm{CH}_{2}\right), 60.06\left(\mathrm{CH}_{3}\right), 60.36$ $\left(\mathrm{CH}_{2}\right), 61.14\left(\mathrm{CH}_{3}\right), 61.27\left(\mathrm{CH}_{3}\right), 61.45\left(2 \times \mathrm{CH}_{3}\right), 61.65\left(\mathrm{CH}_{3}\right), 70.08(\mathrm{CH}), 70.34\left(\mathrm{CH}_{2}\right), 70.57$ $\left(\mathrm{CH}_{2}\right), 70.66\left(\mathrm{CH}_{2}\right), 70.71\left(\mathrm{CH}_{2}\right), 70.88\left(\mathrm{CH}_{2}\right), 70.98(\mathrm{CH}), 71.14(\mathrm{CH}), 71.30(\mathrm{CH}), 71.51(\mathrm{CH})$, $71.86(\mathrm{CH}), 72.43(\mathrm{CH}), 75.96(\mathrm{CH}), 77.02(\mathrm{CH}), 77.14(\mathrm{CH}), 77.56(\mathrm{CH}), 78.17(\mathrm{CH}), 80.10$ $(\mathrm{CH}), 81.36(\mathrm{CH}), 81.39(\mathrm{CH}), 81.49(\mathrm{CH}), 81.58(\mathrm{CH}), 81.63(\mathrm{CH}), 81.88(\mathrm{CH}), 81.99(\mathrm{CH})$, $82.01(\mathrm{CH}), 82.08(\mathrm{CH}), 82.51(\mathrm{CH}), 82.74(\mathrm{CH}), 83.03(\mathrm{CH}), 83.12(\mathrm{CH}), 97.80(\mathrm{CH}), 97.82$ $(\mathrm{CH}), 99.46(2 \times \mathrm{CH}), 99.59(\mathrm{CH}), 100.49(\mathrm{CH}), 101.81(\mathrm{CH}), 104.33(\mathrm{C}), 105.18(\mathrm{C}), 170.01(\mathrm{C})$, 170.12 (C); MS (ESI $-\mathrm{TOF}): m / z(\%) 1539\left[(\mathrm{M}+\mathrm{Na})^{+}, 100\right] ; \mathrm{HRMS}\left(\mathrm{ESI}^{+}-\mathrm{TOF}\right): m / z[\mathrm{M}+\mathrm{Na}]^{+}$ 
calcd for $\mathrm{C}_{65} \mathrm{H}_{112} \mathrm{NaO}_{39}$ 1539.6678; found 1539.6660. Anal. calcd for $\mathrm{C}_{65} \mathrm{H}_{112} \mathrm{O}_{39}: \mathrm{C}, 51.44 ; \mathrm{H}$, 7.44. Found: $\mathrm{C}, 51.48 ; \mathrm{H}, 7.64$. Compound 20: colorless oil, $[\alpha]_{\mathrm{D}}+123.5\left(c 0.96, \mathrm{CHCl}_{3}\right)$; IR (film): 3540, 2928, 1732, 1455, 1368, 1138, 1105, $1046 \mathrm{~cm}^{-1} ;{ }^{1} \mathrm{H}$ NMR $\left(500 \mathrm{MHz}, \mathrm{CDCl}_{3}\right): \delta 2.05(\mathrm{~s}$, 3H), $3.24(\mathrm{dd}, J=10.1,3.5 \mathrm{~Hz}, 1 \mathrm{H}), 3.25(\mathrm{dd}, J=10.1,3.3 \mathrm{~Hz}, 1 \mathrm{H}), 3.35(\mathrm{~s}, 3 \mathrm{H}), 3.37(\mathrm{~s}, 3 \mathrm{H})$, $3.38(\mathrm{~s}, 3 \mathrm{H}), 3.39$ (s, 3H), 3.40 (s, 3H), 3.42 (s, 3H), 3.45 (s, 3H), 3.477 (s, 3H), 3.480 (s, 3H), 3.49 (s, 3H), $3.50(\mathrm{~s}, 3 \mathrm{H}), 3.53(\mathrm{~s}, 3 \mathrm{H}), 3.60(\mathrm{~s}, 3 \mathrm{H}), 3.61(\mathrm{~s}, 6 \mathrm{H}), 3.63(\mathrm{~s}, 3 \mathrm{H}), 3.66(\mathrm{~s}, 3 \mathrm{H}), 3.67$ (s, $3 \mathrm{H}), 3.68(\mathrm{~s}, 3 \mathrm{H}), 3.90(\mathrm{~d}, J=8.8 \mathrm{~Hz}, 1 \mathrm{H}), 4.21(\mathrm{~m}, 1 \mathrm{H}), 4.23(\mathrm{dd}, J=2.5,2.5 \mathrm{~Hz}, 1 \mathrm{H}), 4.27(\mathrm{br}$ dd, $J=11.7,6.3 \mathrm{~Hz}, 1 \mathrm{H}), 4.98(\mathrm{~d}, J=3.2 \mathrm{~Hz}, 1 \mathrm{H}), 5.00(\mathrm{~d}, J=3.2 \mathrm{~Hz}, 1 \mathrm{H}), 5.04(\mathrm{~d}, J=3.5 \mathrm{~Hz}$, $1 \mathrm{H}), 5.09(\mathrm{~d}, J=4.1 \mathrm{~Hz}, 1 \mathrm{H}), 5.13(\mathrm{~d}, J=3.5 \mathrm{~Hz}, 1 \mathrm{H}), 5.15(\mathrm{~d}, J=1.9 \mathrm{~Hz}, 1 \mathrm{H}), 5.17(\mathrm{~d}, J=3.5$ $\mathrm{Hz}, 1 \mathrm{H}) ;{ }^{1} \mathrm{H} \mathrm{NMR}\left(500 \mathrm{MHz}, \mathrm{CDCl}_{3}, 1 \mathrm{D}-\mathrm{TOCSY}\right.$, irradiation at $\left.\mathrm{H} 1^{\mathrm{II}}, 5.17 \mathrm{ppm}\right): \delta 3.24$ (dd, $J=$ 9.9, 3.7 Hz, 1H, H2 $\left.{ }^{\mathrm{II}}\right), 3.64\left(\mathrm{dd}, J=9.2,9.2 \mathrm{~Hz}, 1 \mathrm{H}, \mathrm{H} 3^{\mathrm{II}}\right), 3.90\left(\mathrm{~d}, J=9.0 \mathrm{~Hz}, 1 \mathrm{H}, \mathrm{H} 4^{\mathrm{II}}\right) ;{ }^{1} \mathrm{H}$ NMR (500 MHz, $\mathrm{CDCl}_{3}, 1 \mathrm{D}-\mathrm{TOCSY}$, irradiation at $\left.\mathrm{H}^{\mathrm{I}}, 5.00 \mathrm{ppm}\right): \delta 3.25\left(\mathrm{dd}, J=9.3,3.0 \mathrm{~Hz}, 1 \mathrm{H}, \mathrm{H} 2^{\mathrm{I}}\right)$, $3.54\left(\mathrm{~m}, 1 \mathrm{H}, \mathrm{H} 6^{\mathrm{I}}\right), 3.59\left(\mathrm{dd}, J=9.4,9.4 \mathrm{~Hz}, 1 \mathrm{H}, \mathrm{H} 3^{\mathrm{I}}\right), 3.63\left(\mathrm{dd}, J=9.1,9.1 \mathrm{~Hz}, 1 \mathrm{H}, \mathrm{H} 4^{\mathrm{I}}\right), 4.21(\mathrm{br}$ d, $\left.J=10 \mathrm{~Hz}, 1 \mathrm{H}, \mathrm{H} 5^{\mathrm{I}}\right), 4.27(\mathrm{br} \mathrm{dd}, J=12.6,5.4 \mathrm{~Hz}, 1 \mathrm{H}, \mathrm{H} 6$ ) $){ }^{1} \mathrm{H}$ NMR $\left(500 \mathrm{MHz}, \mathrm{CDCl}_{3}, 1 \mathrm{D}-\right.$ ROESY, irradiation at $\left.\mathrm{H}^{\mathrm{I}}, 5.00 \mathrm{ppm}\right): \delta 3.25\left(\mathrm{dd}, J=9.3,3.3 \mathrm{~Hz}, 1 \mathrm{H}, \mathrm{H} 2^{\mathrm{I}}\right), 3.90(\mathrm{~d}, J=9.1 \mathrm{~Hz}$, $\left.1 \mathrm{H}, \mathrm{H} 4{ }^{\mathrm{II}}\right) ;{ }^{13} \mathrm{C} \mathrm{NMR}\left(125.7 \mathrm{MHz}, \mathrm{CDCl}_{3}\right): \delta 22.05\left(\mathrm{CH}_{3}\right), 57.42\left(\mathrm{CH}_{3}\right), 57.56\left(2 \times \mathrm{CH}_{3}\right), 58.30$ $\left(\mathrm{CH}_{3}\right), 58.39\left(\mathrm{CH}_{3}\right), 58.88\left(\mathrm{CH}_{3}\right), 58.90\left(\mathrm{CH}_{3}\right), 58.93\left(\mathrm{CH}_{3}\right), 58.98\left(\mathrm{CH}_{3}\right), 59.01\left(\mathrm{CH}_{3}\right), 59.04(3 \times$ $\left.\mathrm{CH}_{3}\right), 60.13\left(\mathrm{CH}_{2}\right), 61.13\left(2 \times \mathrm{CH}_{3}\right), 61.40\left(\mathrm{CH}_{3}\right), 61.61\left(\mathrm{CH}_{3}\right), 61.69\left(\mathrm{CH}_{3}\right), 62.06\left(\mathrm{CH}_{3}\right), 63.85$ $\left(\mathrm{CH}_{2}\right), 67.06(\mathrm{CH}), 70.48\left(\mathrm{CH}_{2}\right), 70.74(\mathrm{CH}), 70.77\left(\mathrm{CH}_{2}\right), 70.90(\mathrm{CH}), 70.90\left(\mathrm{CH}_{2}\right), 71.03\left(\mathrm{CH}_{2}\right)$, $71.08(\mathrm{CH}), 71.43(\mathrm{CH}), 72.16\left(\mathrm{CH}_{2}\right), 72.83(\mathrm{CH}), 75.62(\mathrm{CH}), 76.57(\mathrm{CH}), 78.46(\mathrm{CH}), 79.02$ $(\mathrm{CH}), 80.24(\mathrm{CH}), 80.32(\mathrm{CH}), 80.35(\mathrm{CH}), 80.63(\mathrm{CH}), 81.03(\mathrm{CH}), 81.48(\mathrm{CH}), 81.57(\mathrm{CH})$, $81.69(2 \times \mathrm{CH}), 81.73(\mathrm{CH}), 81.77(2 \times \mathrm{CH}), 82.21(\mathrm{CH}), 82.71(\mathrm{CH}), 83.09(\mathrm{CH}), 83.38(\mathrm{CH})$, $97.20(\mathrm{CH}), 97.31(\mathrm{CH}), 98.31(\mathrm{CH}), 98.45(\mathrm{CH}), 99.61(\mathrm{CH}), 100.31(\mathrm{CH}), 100.35(\mathrm{C}), 102.03$ 
(CH), $105.25(\mathrm{C}), 170.26(\mathrm{C})$; MS (ESI $\left.{ }^{+}-\mathrm{TOF}\right): m / z(\%) 1479\left[(\mathrm{M}+\mathrm{Na})^{+}, 100\right]$; HRMS (ESI ${ }^{+}-$ TOF): $m / z[\mathrm{M}+\mathrm{Na}]^{+}$calcd for $\mathrm{C}_{63} \mathrm{H}_{108} \mathrm{NaO}_{37}$ 1479.6467; found 1479.6483. Anal. calcd for $\mathrm{C}_{63} \mathrm{H}_{108} \mathrm{O}_{37}: \mathrm{C}, 51.92 ; \mathrm{H}, 7.47$. Found: $\mathrm{C}, 52.16 ; \mathrm{H}, 7.75$. Compound 21: colorless oil, $[\alpha]_{D}+110.6$ (c 0.36, $\mathrm{CHCl}_{3}$ ); IR (film): 3526, 2928, 1732, 1455, 1368, 1143, 1107, $1046 \mathrm{~cm}^{-1} ;{ }^{1} \mathrm{H}$ NMR (500 $\left.\mathrm{MHz}, \mathrm{CDCl}_{3}\right): \delta 2.06(\mathrm{~s}, 3 \mathrm{H}), 3.12(\mathrm{dd}, J=10.1,3.5 \mathrm{~Hz}, 1 \mathrm{H}), 3.15(\mathrm{dd}, J=9.1,3.2 \mathrm{~Hz}, 1 \mathrm{H}), 3.20$ (dd, $J=9.8,3.8 \mathrm{~Hz}, 1 \mathrm{H}), 3.35(\mathrm{~s}, 3 \mathrm{H}), 3.37$ (s, 9H), $3.40(\mathrm{~s}, 3 \mathrm{H}), 3.43(\mathrm{~s}, 3 \mathrm{H}), 3.46(\mathrm{~s}, 3 \mathrm{H}), 3.47$ (s, 6H), $3.48(\mathrm{~s}, 3 \mathrm{H}), 3.51(\mathrm{~s}, 3 \mathrm{H}), 3.56(\mathrm{~s}, 3 \mathrm{H}), 3.61(\mathrm{~s}, 9 \mathrm{H}), 3.64(\mathrm{~s}, 3 \mathrm{H}), 3.64(\mathrm{~s}, 3 \mathrm{H}), 3.66(\mathrm{~s}$, 3H), 3.67 (s, 3H), $3.82\left(\mathrm{dd}, J=10.7,10.7 \mathrm{~Hz}, 1 \mathrm{H}, \mathrm{H}^{\mathrm{I}}\right), 3.95$ (d, $\left.J=9.8 \mathrm{~Hz}, 1 \mathrm{H}, \mathrm{H} 6^{\mathrm{II}}\right), 4.06$ (d, $J=$ $9.8 \mathrm{~Hz}, 1 \mathrm{H}, \mathrm{H} 6^{\mathrm{II}}$ ), 4.11 (br s, 2H, H4 ${ }^{\mathrm{II}}$ and $\mathrm{H} 3^{\mathrm{II}}$ ), 4.37 (ddd, $\left.J=12.0,6.6,1.6 \mathrm{~Hz}, 1 \mathrm{H}, \mathrm{H} 6^{\mathrm{III}}\right), 5.01$ (d, $J=3.2 \mathrm{~Hz}, 1 \mathrm{H}), 5.03(\mathrm{~d}, J=3.5 \mathrm{~Hz}, 1 \mathrm{H}), 5.04(\mathrm{~d}, J=4.4 \mathrm{~Hz}, 1 \mathrm{H}), 5.08(\mathrm{~d}, J=1.9 \mathrm{~Hz}, 1 \mathrm{H})$, $5.163(\mathrm{~d}, J=3.2 \mathrm{~Hz}, 1 \mathrm{H}), 5.166(\mathrm{~d}, J=2.9 \mathrm{~Hz}, 1 \mathrm{H}), 5.21(\mathrm{~d}, J=3.8 \mathrm{~Hz}, 1 \mathrm{H}) ;{ }^{1} \mathrm{H}$ NMR $(500 \mathrm{MHz}$, $\mathrm{CDCl}_{3}, 1 \mathrm{D}-\mathrm{TOCSY}$, irradiation at H1 ${ }^{\mathrm{II}}, 5.08 \mathrm{ppm}$ ): $\delta 3.24$ (br s, $\left.1 \mathrm{H}, \mathrm{H} 2^{\mathrm{II}}\right), 4.109$ (dd, $J=2.9,2.9$ $\left.\mathrm{Hz}, 1 \mathrm{H}, \mathrm{H} 3^{\mathrm{II}}\right), 4.111\left(\mathrm{~d}, J=2.9 \mathrm{~Hz}, 1 \mathrm{H}, \mathrm{H} 4{ }^{\mathrm{II}}\right) ;{ }^{1} \mathrm{H}$ NMR $\left(500 \mathrm{MHz}, \mathrm{CDCl}_{3}, 1 \mathrm{D}-\mathrm{TOCSY}\right.$, irradiation at $\left.\mathrm{H}^{\mathrm{I}}, 5.01 \mathrm{ppm}\right): \delta 3.16\left(\mathrm{br} \mathrm{d}, J=10.1 \mathrm{~Hz}, 1 \mathrm{H}, \mathrm{H} 2^{\mathrm{I}}\right), 3.42\left(\mathrm{dd}, J=9.3,9.3 \mathrm{~Hz}, 1 \mathrm{H}, \mathrm{H} 3^{\mathrm{I}}\right), 3.46(\mathrm{dd}$, $\left.J=9.0,9.0 \mathrm{~Hz}, 1 \mathrm{H}, \mathrm{H} 4^{\mathrm{I}}\right), 3.65\left(\mathrm{~m}, 1 \mathrm{H}, \mathrm{H} 5^{\mathrm{I}}\right), 3.98\left(\mathrm{dd}, J=9.2,2.8 \mathrm{~Hz}, 1 \mathrm{H}, \mathrm{H} 6{ }^{\mathrm{I}}\right) ;{ }^{1} \mathrm{H}$ NMR $(500$ $\mathrm{MHz}, \mathrm{CDCl}_{3}, 1 \mathrm{D}-\mathrm{ROESY}$, irradiation at $\left.\mathrm{H} 1^{\mathrm{I}}, 5.01 \mathrm{ppm}\right): \delta 4.11\left(\mathrm{~d}, J=2.9 \mathrm{~Hz}, 1 \mathrm{H}, \mathrm{H} 4{ }^{\mathrm{II}}\right) ;{ }^{13} \mathrm{C} \mathrm{NMR}$ (125.7 MHz, $\left.\mathrm{CDCl}_{3}\right): \delta 22.13\left(\mathrm{CH}_{3}\right), 57.08\left(\mathrm{CH}_{3}\right), 57.54\left(\mathrm{CH}_{3}\right), 57.84\left(\mathrm{CH}_{3}\right), 58.14\left(\mathrm{CH}_{3}\right), 58.20$ $\left(\mathrm{CH}_{3}\right), 58.84\left(\mathrm{CH}_{3}\right), 58.86\left(\mathrm{CH}_{3}\right), 58.89\left(\mathrm{CH}_{3}\right), 58.98\left(\mathrm{CH}_{3}\right), 59.04\left(\mathrm{CH}_{3}\right), 59.10\left(\mathrm{CH}_{3}\right), 59.14$ $\left(\mathrm{CH}_{3}\right), 59.89\left(\mathrm{CH}_{3}\right), 60.37\left(\mathrm{CH}_{2}\right), 60.90\left(\mathrm{CH}_{3}\right), 61.25\left(\mathrm{CH}_{3}\right), 61.35\left(\mathrm{CH}_{3}\right), 61.67\left(\mathrm{CH}_{3}\right), 61.73$ $\left(\mathrm{CH}_{3}\right), 62.10\left(\mathrm{CH}_{3}\right), 63.91\left(\mathrm{CH}_{2}\right), 67.13(\mathrm{CH}), 69.00(\mathrm{CH}), 70.29\left(\mathrm{CH}_{2}\right), 70.45(\mathrm{CH}), 70.95(\mathrm{CH})$, $70.95\left(\mathrm{CH}_{2}\right), 71.33(\mathrm{CH}), 71.47\left(\mathrm{CH}_{2}\right), 71.54(\mathrm{CH}), 71.54\left(\mathrm{CH}_{2}\right), 71.82\left(\mathrm{CH}_{2}\right), 75.65(\mathrm{CH}), 76.98$ $(\mathrm{CH}), 77.20(\mathrm{CH}), 77.55(\mathrm{CH}), 79.22(\mathrm{CH}), 79.90(\mathrm{CH}), 80.36(\mathrm{CH}), 80.86(\mathrm{CH}), 80.96(\mathrm{CH})$, $80.99(\mathrm{CH}), 81.07(\mathrm{CH}), 81.36(\mathrm{CH}), 81.79(\mathrm{CH}), 81.87(\mathrm{CH}), 81.95(\mathrm{CH}), 81.97(\mathrm{CH}), 82.34$ 
$(\mathrm{CH}), 82.87(\mathrm{CH}), 83.09(\mathrm{CH}), 83.45(\mathrm{CH}), 97.08(\mathrm{CH}), 97.66(\mathrm{CH}), 98.06(\mathrm{CH}), 98.21(\mathrm{CH})$, $98.77(\mathrm{CH}), 99.10(\mathrm{CH}), 99.28(\mathrm{CH}), 100.76(\mathrm{C}), 105.18(\mathrm{C}), 170.26(\mathrm{C})$; MS (ESI $\left.{ }^{+}-\mathrm{TOF}\right): \mathrm{m} / \mathrm{z}$ (\%) $1479\left[(\mathrm{M}+\mathrm{Na})^{+}, 100\right]$; HRMS (ESI $\left.{ }^{+}-\mathrm{TOF}\right): \mathrm{m} / z[\mathrm{M}+\mathrm{Na}]^{+}$calcd for $\mathrm{C}_{63} \mathrm{H}_{108} \mathrm{NaO}_{37}$ 1479.6467; found 1479.6459. Anal. calcd for $\mathrm{C}_{63} \mathrm{H}_{108} \mathrm{O}_{37}$ : C, 51.92; H, 7.47. Found: $\mathrm{C}, 52.14 ; \mathrm{H}, 7.39$. Compound 22: colorless oil, $[\alpha]_{\mathrm{D}}+116.9$ ( $c$ 0.46, $\left.\mathrm{CHCl}_{3}\right)$; IR (film): 2928, 1455, 1370, 1159, 1102, $1046 \mathrm{~cm}^{-1} ;{ }^{1} \mathrm{H}$ NMR $\left(500 \mathrm{MHz}, \mathrm{CDCl}_{3}\right): \delta 3.099(\mathrm{~d}, J=9.8 \mathrm{~Hz}, 1 \mathrm{H}), 3.10(\mathrm{dd}, J=9.5,3.5 \mathrm{~Hz}$, 1H), 3.24 (dd, $J=9.8,9.8 \mathrm{~Hz}, 1 \mathrm{H}), 3.30(\mathrm{dd}, J=9.5,3.5 \mathrm{~Hz}, 1 \mathrm{H}), 3.34$ (s, 3H), 3.37 (s, 3H), 3.38 (s, 6H), 3.39 (s, 3H), 3.47 (s, 6H), 3.48 (s, 3H), 3.49 (s, 3H), 3.54 (s, 3H), 3.57 (s, 3H), 3.575 (s, 3H), $3.582(\mathrm{~s}, 3 \mathrm{H}), 3.59(\mathrm{~s}, 6 \mathrm{H}), 3.618(\mathrm{~s}, 3 \mathrm{H}), 3.619(\mathrm{~s}, 3 \mathrm{H}), 3.70(\mathrm{~s}, 3 \mathrm{H}), 3.74(\mathrm{~s}, 3 \mathrm{H}), 4.24(\mathrm{dd}$, $J=9.1,7.9 \mathrm{~Hz}, 1 \mathrm{H}), 4.30(\mathrm{ddd}, J=10.7,7.9,7.9 \mathrm{~Hz}, 1 \mathrm{H}), 5.02(\mathrm{~d}, J=3.5 \mathrm{~Hz}, 1 \mathrm{H}), 5.06(\mathrm{~d}, J=3.5$ $\mathrm{Hz}, 1 \mathrm{H}), 5.09(\mathrm{~d}, J=3.2 \mathrm{~Hz}, 1 \mathrm{H}), 5.094(\mathrm{~d}, J=3.2 \mathrm{~Hz}, 1 \mathrm{H}), 5.23(\mathrm{~d}, J=3.5 \mathrm{~Hz}, 1 \mathrm{H}), 5.30(\mathrm{~d}, J=$ $3.8 \mathrm{~Hz}, 1 \mathrm{H}) ;{ }^{1} \mathrm{H} \mathrm{NMR}\left(500 \mathrm{MHz}, \mathrm{CDCl}_{3}, 1 \mathrm{D}-\mathrm{TOCSY}\right.$, irradiation at $\left.\mathrm{H} 1{ }^{1 \mathrm{II}}, 5.30 \mathrm{ppm}\right): \delta 3.20(\mathrm{dd}$, $J=9.6,3.8 \mathrm{~Hz}, 1 \mathrm{H}, \mathrm{H} 2^{\mathrm{III}}$ ), 3.59 (dd, $\left.J=9.2,9.2 \mathrm{~Hz}, 1 \mathrm{H}, \mathrm{H} 3^{\mathrm{III}}\right), 3.64$ (dd, $J=9.4,9.4 \mathrm{~Hz}, 1 \mathrm{H}, \mathrm{H} 6^{\mathrm{II}}$ ), $3.68\left(\mathrm{dd}, J=9.2,9.2 \mathrm{~Hz}, 1 \mathrm{H}, \mathrm{H} 4^{\mathrm{III}}\right), 4.24\left(\mathrm{dd}, J=9.0,9.0 \mathrm{~Hz}, 1 \mathrm{H}, \mathrm{H} 6^{\mathrm{III}}\right), 3.99$ (ddd, $J=10.4,8.0$, $\left.8.0 \mathrm{~Hz}, 1 \mathrm{H}, \mathrm{H} 5^{\mathrm{III}}\right) ;{ }^{1} \mathrm{H}$ NMR (500 MHz, $\mathrm{CDCl}_{3}, 1 \mathrm{D}-\mathrm{TOCSY}$, irradiation at $\mathrm{H} 1^{\mathrm{I}}, 5.23 \mathrm{ppm}$ ): $\delta 3.31$ $\left(\mathrm{dd}, J=9.3,3.9 \mathrm{~Hz}, 1 \mathrm{H}, \mathrm{H} 2^{\mathrm{I}}\right), 3.78\left(\mathrm{dd}, J=9.6,9.6 \mathrm{~Hz}, 1 \mathrm{H}, \mathrm{H} 3^{\mathrm{I}}\right), 3.85\left(\mathrm{~d}, J=9.5 \mathrm{~Hz}, 1 \mathrm{H}, \mathrm{H} 4^{\mathrm{I}}\right) ;{ }^{1} \mathrm{H}$ NMR (500 MHz, $\mathrm{CDCl}_{3}, 1 \mathrm{D}-\mathrm{ROESY}$, irradiation at $\left.\mathrm{H1}^{\mathrm{IIII}}, 5.30 \mathrm{ppm}\right): \delta 3.21$ (dd, $J=9.7,3.8 \mathrm{~Hz}$, $\left.1 \mathrm{H}, \mathrm{H} 2{ }^{\mathrm{III}}\right), 3.60\left(\mathrm{dd}, J=9.2,9.2 \mathrm{~Hz}, 1 \mathrm{H}, \mathrm{H}^{\mathrm{IV}}\right) ;{ }^{13} \mathrm{C} \mathrm{NMR}\left(125.7 \mathrm{MHz}, \mathrm{CDCl}_{3}\right): \delta 57.84\left(\mathrm{CH}_{3}\right)$, $57.88\left(\mathrm{CH}_{3}\right), 58.02\left(\mathrm{CH}_{3}\right), 58.26\left(\mathrm{CH}_{3}\right), 58.34\left(\mathrm{CH}_{3}\right), 58.81\left(\mathrm{CH}_{3}\right), 58.83\left(\mathrm{CH}_{3}\right), 58.94\left(\mathrm{CH}_{3}\right), 58.98$ $\left(\mathrm{CH}_{3}\right), 59.38\left(\mathrm{CH}_{3}\right), 59.89\left(\mathrm{CH}_{3}\right), 60.75\left(2 \times \mathrm{CH}_{3}\right), 61.01\left(\mathrm{CH}_{3}\right), 61.10\left(\mathrm{CH}_{3}\right), 61.50\left(\mathrm{CH}_{3}\right), 61.85$ $\left(\mathrm{CH}_{3}\right), 61.96\left(\mathrm{CH}_{3}\right), 62.28\left(\mathrm{CH}_{3}\right), 62.53(\mathrm{CH}), 62.79\left(\mathrm{CH}_{2}\right), 65.12\left(\mathrm{CH}_{2}\right), 66.50(\mathrm{CH}), 70.27(\mathrm{CH})$, $70.96\left(\mathrm{CH}_{2}\right), 71.08(\mathrm{CH}), 71.11(\mathrm{CH}), 71.16\left(\mathrm{CH}_{2}\right), 71.42\left(\mathrm{CH}_{2}\right), 71.73\left(\mathrm{CH}_{2}\right), 71.94(\mathrm{CH}), 72.18$ $\left(\mathrm{CH}_{2}\right), 74.72(\mathrm{CH}), 77.17(\mathrm{CH}), 77.61(\mathrm{CH}), 78.77(\mathrm{CH}), 79.92(\mathrm{CH}), 80.57(\mathrm{CH}), 80.83(\mathrm{CH})$, 
$81.03(\mathrm{CH}), 81.14(\mathrm{CH}), 81.31(\mathrm{CH}), 81.46(\mathrm{CH}), 81.47(\mathrm{CH}), 81.90(\mathrm{CH}), 82.00(\mathrm{CH}), 82.07$ $(\mathrm{CH}), 82.27(\mathrm{CH}), 82.48(\mathrm{CH}), 82.60(\mathrm{CH}), 83.37(\mathrm{CH}), 83.56(\mathrm{CH}), 83.91(\mathrm{CH}), 96.87(\mathrm{CH})$, $99.44(2 \times \mathrm{CH}), 99.86(\mathrm{CH}), 100.34(\mathrm{CH}), 100.47(\mathrm{CH}), 101.28(\mathrm{C}), 111.08(\mathrm{C}) ; \mathrm{MS}\left(\mathrm{ESI}^{+}-\mathrm{TOF}\right)$ : $m / z(\%) 1419\left[(\mathrm{M}+\mathrm{Na})^{+}, 100\right]$; HRMS $\left(\mathrm{ESI}^{+}-\mathrm{TOF}\right): m / z[\mathrm{M}+\mathrm{Na}]^{+}$calcd for $\mathrm{C}_{61} \mathrm{H}_{104} \mathrm{NaO}_{35}$ 1419.6256; found 1419.6287. Anal. calcd for $\mathrm{C}_{61} \mathrm{H}_{104} \mathrm{O}_{35}: \mathrm{C}, 52.43 ; \mathrm{H}, 7.50$. Found: $\mathrm{C}, 52.18 ; \mathrm{H}$, 7.74 .

Oxidative HAT of $2^{I-V I}, 3^{I-V I}, 6^{I I, I V-V I}$-hexadeca-O-methyl- $\alpha$-cyclomaltohexaose (23). A solution of alcohol $23(120 \mathrm{mg}, 0.10 \mathrm{mmol})$ in dry $\mathrm{CH}_{2} \mathrm{Cl}_{2}(4 \mathrm{~mL})$ containing DIB (71 mg, $\left.0.22 \mathrm{mmol}\right)$ and $\mathrm{I}_{2}$ (25.4 mg, $0.10 \mathrm{mmol}$ ) was stirred under nitrogen at $28^{\circ} \mathrm{C}$ for $1.5 \mathrm{~h}$ while irradiated with two $80 \mathrm{~W}$ tungsten-filament lamps. The reaction mixture was then directly loaded onto a silica gel (TLC Silica gel $60 \mathrm{~F}_{254}$, scraped from Merck Aluminum sheets) column chromatography (hexanes-acetone, $65: 35 \rightarrow 60: 40) \quad$ to $\quad$ give $\quad$ cyclo- $5^{\mathrm{II}}, 6^{\mathrm{III}}$-anhydro- $\left(5^{\mathrm{II}} R\right)-(2,3,6$-tri- $O$-methyl- $\alpha$-D-xylo-hexos-5-

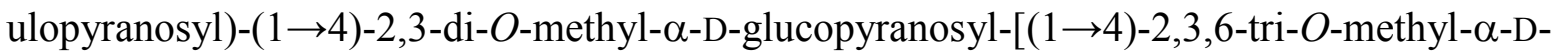
glucopyranosyl $]_{2}-(1 \rightarrow 4)-5^{\mathrm{VI}}, 6^{\mathrm{I}}$-anhydro-( $\left.5^{\mathrm{VI}} R\right)-(2,3,6$-tri- $O$-methyl- $\alpha$-D-xylo-hexos-5ulopyranosyl)-(1 $\rightarrow 4$ )-2,3-di-O-methyl- $\alpha$-D-glucopyranosyl (24) (33.4 mg, 0.028, 28\%), cyclo$5^{\mathrm{II}}, 6^{\mathrm{III}}$-anhydro-(5R)-(2,3,6-tri- $O$-methyl- $\alpha$-D-xylo-hexos-5-ulopyranosyl)-(1 $\left.\rightarrow 4\right)$-2,3-di-Omethyl- $\alpha$-D-glucopyranosyl-[( $\rightarrow 4$ )-2,3,6-tri- $O$-methyl- $\alpha$-D-glucopyranosyl] $3-(1 \rightarrow 4)-2,3$-di- $O$ methyl- $\alpha$-D-glucopyranosyl (25) (22 mg, $0.018 \mathrm{mmol}, 18 \%)$, and cyclo- $5^{\mathrm{VI}}, 6^{\mathrm{I}}$-anhydro-( $\left.5^{\mathrm{VI}} R\right)$ (2,3,6-tri- $O$-methyl- $\alpha$-D-xylo-hexos-5-ulopyranosyl)-(1 $\rightarrow 4)-2,3$-di- $O$-methyl- $\alpha$-Dglucopyranosyl)-(1 $\rightarrow 4$ )-2,3,6-tri-O-methyl- $\alpha$-D-glucopyranosyl-( $1 \rightarrow 4)$-2,3-di-O-methyl- $\alpha$-D-

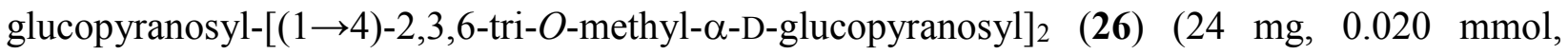
20\%). Compound 24: colorless oil, $[\alpha]_{\mathrm{D}}+124.1$ (c 1.70, $\mathrm{CHCl}_{3}$ ); IR (film): 2928, 1453, 1368, 1140, 1107, $1060 \mathrm{~cm}^{-1} ;{ }^{1} \mathrm{H}$ NMR (500 MHz, $\left.\mathrm{CDCl}_{3}\right): \delta 3.14(\mathrm{~m}, 4 \mathrm{H}), 3.22(\mathrm{dd}, J=9.1,3.8 \mathrm{~Hz}, 1 \mathrm{H}), 3.24$ 
(dd, $J=9.5,3.5 \mathrm{~Hz}, 1 \mathrm{H}), 3.35(\mathrm{~s}, 3 \mathrm{H}), 3.36(\mathrm{~s}, 6 \mathrm{H}), 3.37$ (s, 3H), $3.466(\mathrm{~s}, 3 \mathrm{H}), 3.469$ (s, 3H), 3.485 (s, 3H), $3.491(\mathrm{~s}, 6 \mathrm{H}), 3.494(\mathrm{~s}, 3 \mathrm{H}), 3.60(\mathrm{~s}, 3 \mathrm{H}), 3.62(\mathrm{~s}, 3 \mathrm{H}), 3.63(\mathrm{~s}, 3 \mathrm{H}), 3.68(\mathrm{~s}, 3 \mathrm{H}), 3.70(\mathrm{~s}$, 3H), $3.71(\mathrm{~s}, 3 \mathrm{H}), 4.98(\mathrm{~d}, J=3.8 \mathrm{~Hz}, 1 \mathrm{H}), 5.00(\mathrm{~d}, J=3.2 \mathrm{~Hz}, 2 \mathrm{H}), 5.03(\mathrm{~d}, J=3.8 \mathrm{~Hz}, 1 \mathrm{H}), 5.15$ $(\mathrm{d}, J=3.5 \mathrm{~Hz}, 1 \mathrm{H}), 5.16(\mathrm{~d}, J=3.5 \mathrm{~Hz}, 1 \mathrm{H}) ;{ }^{13} \mathrm{C} \mathrm{NMR}\left(125.7 \mathrm{MHz}, \mathrm{CDCl}_{3}\right): \delta 57.59\left(\mathrm{CH}_{3}\right), 57.70$ $\left(\mathrm{CH}_{3}\right), 57.92\left(\mathrm{CH}_{3}\right), 58.11\left(\mathrm{CH}_{3}\right), 58.38\left(\mathrm{CH}_{3}\right), 58.62\left(\mathrm{CH}_{3}\right), 58.76\left(\mathrm{CH}_{3}\right), 58.95\left(\mathrm{CH}_{3}\right), 59.09$ $\left(\mathrm{CH}_{3}\right), 59.14\left(\mathrm{CH}_{3}\right), 61.27\left(\mathrm{CH}_{3}\right), 61.32\left(\mathrm{CH}_{3}\right), 61.52\left(\mathrm{CH}_{3}\right), 61.86\left(\mathrm{CH}_{3}\right), 61.94\left(\mathrm{CH}_{3}\right), 62.14$ $\left(\mathrm{CH}_{3}\right), 63.44\left(\mathrm{CH}_{2}\right), 63.91\left(\mathrm{CH}_{2}\right), 65.60(\mathrm{CH}), 66.37(\mathrm{CH}), 70.48(\mathrm{CH}), 71.03(\mathrm{CH}), 71.57\left(\mathrm{CH}_{2}\right)$, $71.67\left(\mathrm{CH}_{2}\right), 71.81\left(\mathrm{CH}_{2}\right), 72.90\left(\mathrm{CH}_{2}\right), 78.05(\mathrm{CH}), 78.23(\mathrm{CH}), 79.77(\mathrm{CH}), 79.91(\mathrm{CH}), 80.07$ $(\mathrm{CH}), 80.74(\mathrm{CH}), 80.82(\mathrm{CH}), 80.97(\mathrm{CH}), 81.14(\mathrm{CH}), 81.16(\mathrm{CH}), 81.19(\mathrm{CH}), 81.23(\mathrm{CH})$, $81.34(\mathrm{CH}), 81.79(\mathrm{CH}), 82.37(\mathrm{CH}), 82.44(\mathrm{CH}), 82.60(\mathrm{CH}), 83.29(\mathrm{CH}), 96.36(\mathrm{CH}), 96.48$ (CH), $97.37(\mathrm{CH}), 97.53(\mathrm{CH}), 100.15(\mathrm{CH}), 100.21(\mathrm{CH}), 100.47(\mathrm{C}), 100.78(\mathrm{C})$; MS (ESI ${ }^{+}$ TOF): $m / z(\%) 1215$ [(M+ Na) $\left.)^{+}, 100\right]$; HRMS (ESI ${ }^{+}$-TOF): $m / z[\mathrm{M}+\mathrm{Na}]^{+}$calcd for $\mathrm{C}_{52} \mathrm{H}_{88} \mathrm{NaO}_{30}$ 1215.5258; found 1215.5237. Anal. calcd for $\mathrm{C}_{52} \mathrm{H}_{88} \mathrm{O}_{30}$ : C, 52.34; H, 7.43. Found: $\mathrm{C}, 52.42 ; \mathrm{H}$, 7.43. Compound 25: colorless oil, $[\alpha]_{\mathrm{D}}+123.7$ (c 1.81, $\left.\mathrm{CHCl}_{3}\right)$; IR (film): 3494, 2933, 1454, 1365, $1109,1039 \mathrm{~cm}^{-1} ;{ }^{1} \mathrm{H}$ NMR (500 MHz, $\left.\mathrm{CDCl}_{3}\right): \delta 3.11(\mathrm{dd}, J=10.1,3.5 \mathrm{~Hz}, 1 \mathrm{H}), 3.13(\mathrm{dd}, J=$ $10.4,3.5 \mathrm{~Hz}, 1 \mathrm{H}), 3.15(\mathrm{dd}, J=9.8,3.2 \mathrm{~Hz}, 1 \mathrm{H}), 3.18(\mathrm{dd}, J=10.1,3.5 \mathrm{~Hz}, 1 \mathrm{H}), 3.20(\mathrm{dd}, J=9.8$, $3.5 \mathrm{~Hz}, 1 \mathrm{H}), 3.25$ (dd, $J=9.6,3.3 \mathrm{~Hz}, 1 \mathrm{H}), 3.35$ (s, 3H), 3.369 (s, 3H), 3.371 (s, 3H), 3.38 (s, 3H), $3.476(\mathrm{~s}, 3 \mathrm{H}), 3.479(\mathrm{~s}, 3 \mathrm{H}), 3.487(\mathrm{~s}, 3 \mathrm{H}), 3.490(\mathrm{~s}, 6 \mathrm{H}), 3.495(\mathrm{~s}, 3 \mathrm{H}), 3.58(\mathrm{~s}, 3 \mathrm{H}), 3.60(\mathrm{~s}, 3 \mathrm{H})$, $3.638(\mathrm{~s}, 3 \mathrm{H}), 3.644(\mathrm{~s}, 3 \mathrm{H}), 3.70(\mathrm{~s}, 3 \mathrm{H}), 3.71(\mathrm{~s}, 3 \mathrm{H}), 4.98(\mathrm{~d}, J=3.8 \mathrm{~Hz}, 1 \mathrm{H}), 4.99$ (d, $J=3.8$ $\mathrm{Hz}, 1 \mathrm{H}), 5.00(\mathrm{~d}, J=3.5 \mathrm{~Hz}, 1 \mathrm{H}), 5.01(\mathrm{~d}, J=3.5 \mathrm{~Hz}, 1 \mathrm{H}), 5.08(\mathrm{~d}, J=3.5 \mathrm{~Hz}, 1 \mathrm{H}), 5.17(\mathrm{~d}, J=$ $3.2 \mathrm{~Hz}, 1 \mathrm{H}) ;{ }^{13} \mathrm{C} \mathrm{NMR}\left(125.7 \mathrm{MHz}, \mathrm{CDCl}_{3}\right): \delta 57.71\left(\mathrm{CH}_{3}\right), 57.84\left(2 \times \mathrm{CH}_{3}\right), 58.17\left(\mathrm{CH}_{3}\right), 58.42$ $\left(\mathrm{CH}_{3}\right), 58.49\left(\mathrm{CH}_{3}\right), 58.95\left(2 \times \mathrm{CH}_{3}\right), 59.00\left(\mathrm{CH}_{3}\right), 59.14\left(\mathrm{CH}_{3}\right), 61.28\left(\mathrm{CH}_{3}\right), 61.33\left(\mathrm{CH}_{3}\right), 61.61$ $\left(\mathrm{CH}_{3}\right), 61.90\left(\mathrm{CH}_{3}\right), 61.94\left(\mathrm{CH}_{3}\right), 62.06\left(\mathrm{CH}_{2}\right), 62.20\left(\mathrm{CH}_{3}\right), 63.76\left(\mathrm{CH}_{2}\right), 66.25(\mathrm{CH}), 70.96(\mathrm{CH})$, 
$71.05\left(\mathrm{CH}_{2}\right), 71.40(\mathrm{CH}), 71.52\left(\mathrm{CH}_{2}\right), 71.62(\mathrm{CH}), 72.12(\mathrm{CH}), 72.12\left(\mathrm{CH}_{2}\right), 72.22\left(\mathrm{CH}_{2}\right), 78.45$ $(\mathrm{CH}), 80.44(2 \times \mathrm{CH}), 80.81(\mathrm{CH}), 80.94(\mathrm{CH}), 81.01(\mathrm{CH}), 81.06(\mathrm{CH}), 81.19(\mathrm{CH}), 81.35(\mathrm{CH})$, $81.44(\mathrm{CH}), 81.76(\mathrm{CH}), 81.93(\mathrm{CH}), 82.09(\mathrm{CH}), 82.12(\mathrm{CH}), 82.33(2 \times \mathrm{CH}), 82.55(\mathrm{CH}), 82.59$ $(\mathrm{CH}), 97.44(\mathrm{CH}), 97.80(\mathrm{CH}), 98.64(\mathrm{CH}), 99.10(\mathrm{CH}), 99.78(\mathrm{CH}), 100.04(\mathrm{CH}), 100.69(\mathrm{C})$; MS (ESI'-TOF): $m / z(\%) 1217\left[(\mathrm{M}+\mathrm{Na})^{+}, 100\right]$; HRMS $\left(\mathrm{ESI}^{+}-\mathrm{TOF}\right): m / z[\mathrm{M}+\mathrm{Na}]^{+}$calcd for $\mathrm{C}_{52} \mathrm{H}_{90} \mathrm{NaO}_{30}$ 1217.5415; found 1217.5405. Anal. calcd for $\mathrm{C}_{52} \mathrm{H}_{90} \mathrm{O}_{30}$ : C, 52.25; H, 7.59. Found: C, 51.98; H, 7.48. Compound 26: colorless oil, $[\alpha]_{\mathrm{D}}+112.0$ (c 1.65, $\left.\mathrm{CHCl}_{3}\right)$; IR (film): 3475, 2933, 1454, 1367, 1111, $1044 \mathrm{~cm}^{-1} ;{ }^{1} \mathrm{H}$ NMR (500 MHz, $\left.\mathrm{CDCl}_{3}\right): \delta 3.10(\mathrm{dd}, J=10.1,3.5 \mathrm{~Hz}, 1 \mathrm{H}), 3.13$ (dd, $J=10.1,3.5 \mathrm{~Hz}, 1 \mathrm{H}), 3.15(\mathrm{dd}, J=9.2,3.8 \mathrm{~Hz}, 1 \mathrm{H}), 3.18(\mathrm{dd}, J=9.8,3.8 \mathrm{~Hz}, 1 \mathrm{H}), 3.19(\mathrm{dd}$, $J=9.8,3.2 \mathrm{~Hz}, 1 \mathrm{H}), 3.25(\mathrm{dd}, J=9.6,3.3 \mathrm{~Hz}, 1 \mathrm{H}), 3.35(\mathrm{~s}, 3 \mathrm{H}), 3.37(\mathrm{~s}, 3 \mathrm{H}), 3.37$ (s, 3H), $3.38(\mathrm{~s}$, 3H), 3.47 (s, 3H), 3.47 (s, 3H), 3.48 (s, 3H), 3.48 (s, 3H), 3.49 (s, 3H), $3.53(\mathrm{~s}, 3 \mathrm{H}), 3.59$ (s, 3H), $3.60(\mathrm{~s}, 3 \mathrm{H}), 3.63(\mathrm{~s}, 3 \mathrm{H}), 3.63(\mathrm{~s}, 3 \mathrm{H}), 3.70(\mathrm{~s}, 3 \mathrm{H}), 3.74(\mathrm{~s}, 3 \mathrm{H}), 4.96(\mathrm{~d}, J=3.8 \mathrm{~Hz}, 1 \mathrm{H}), 5.01(\mathrm{~d}$, $J=3.8 \mathrm{~Hz}, 1 \mathrm{H}), 5.03(\mathrm{~d}, J=3.5 \mathrm{~Hz}, 1 \mathrm{H}), 5.04(\mathrm{~d}, J=3.2 \mathrm{~Hz}, 1 \mathrm{H}), 5.07(\mathrm{~d}, J=3.5 \mathrm{~Hz}, 1 \mathrm{H}), 5.19$ $(\mathrm{d}, J=3.5 \mathrm{~Hz}, 1 \mathrm{H}) ;{ }^{13} \mathrm{C} \mathrm{NMR}\left(125.7 \mathrm{MHz}, \mathrm{CDCl}_{3}\right): \delta 57.67\left(\mathrm{CH}_{3}\right), 57.82\left(\mathrm{CH}_{3}\right), 57.84\left(\mathrm{CH}_{3}\right)$, $57.99\left(\mathrm{CH}_{3}\right), 58.39\left(\mathrm{CH}_{3}\right), 58.47\left(\mathrm{CH}_{3}\right), 58.80\left(\mathrm{CH}_{3}\right), 59.00\left(2 \times \mathrm{CH}_{3}\right), 59.09\left(\mathrm{CH}_{3}\right), 61.28\left(\mathrm{CH}_{3}\right)$, $61.50\left(\mathrm{CH}_{3}\right), 61.54\left(\mathrm{CH}_{3}\right), 61.93\left(\mathrm{CH}_{3}\right), 61.97\left(\mathrm{CH}_{3}\right), 62.25\left(\mathrm{CH}_{3}\right), 62.48\left(\mathrm{CH}_{2}\right), 63.86\left(\mathrm{CH}_{2}\right), 66.28$ (CH), $70.87(\mathrm{CH}), 71.03(\mathrm{CH}), 71.10(\mathrm{CH}), 71.12\left(\mathrm{CH}_{2}\right), 71.24\left(\mathrm{CH}_{2}\right), 71.83\left(\mathrm{CH}_{2}\right), 71.99\left(\mathrm{CH}_{2}\right)$, $72.77(\mathrm{CH}), 78.30(\mathrm{CH}), 80.46(\mathrm{CH}), 80.57(\mathrm{CH}), 80.73(\mathrm{CH}), 80.78(\mathrm{CH}), 81.02(2 \times \mathrm{CH}), 81.31$ $(\mathrm{CH}), 81.42(\mathrm{CH}), 81.50(\mathrm{CH}), 81.75(\mathrm{CH}), 81.89(\mathrm{CH}), 81.97(\mathrm{CH}), 82.11(\mathrm{CH}), 82.30(\mathrm{CH})$, $82.50(\mathrm{CH}), 82.60(\mathrm{CH}), 82.70(\mathrm{CH}), 97.36(\mathrm{CH}), 97.75(\mathrm{CH}), 98.77(\mathrm{CH}), 99.57(\mathrm{CH}), 99.86$ (CH), $100.04(\mathrm{CH}), 100.80(\mathrm{C})$; MS (ESI ${ }^{+}$-TOF): $m / z(\%) 1217\left[(\mathrm{M}+\mathrm{Na})^{+}, 100\right]$; HRMS (ESI ${ }^{+}-$ TOF): $m / z[\mathrm{M}+\mathrm{Na}]^{+}$calcd for $\mathrm{C}_{52} \mathrm{H}_{90} \mathrm{NaO}_{30}$ 1217.5415; found 1217.5427. Anal. calcd for $\mathrm{C}_{52} \mathrm{H}_{90} \mathrm{O}_{30}: \mathrm{C}, 52.25 ; \mathrm{H}, 7.59$. Found: $\mathrm{C}, 52.30 ; \mathrm{H}, 7.51$. 
Cyclo-5 ${ }^{I I}, 6^{I I I}$-anhydro-( $\left(5^{I I} R\right)-(2,3,6$-tri-O-methyl- $\alpha$-D-xylo-hexos-5-ulopyranosyl)-(1 $\rightarrow 4)-2,3-$ di-O-methyl- $\alpha$-D-glucopyranosyl-[(1 $\rightarrow 4)-2,3,6$-tri-O-methyl- $\alpha$-D-glucopyranosyl] $3-(1 \rightarrow 4)-6^{I}-O$ acetyl-2,3-di-O-methyl- $\alpha$-D-glucopyranosyl (27). A solution of alcohol $25(16.7 \mathrm{mg}, 0.014 \mathrm{mmol})$ in dry pyridine $(0.9 \mathrm{~mL})$ containing $\mathrm{Ac}_{2} \mathrm{O}(0.3 \mathrm{~mL})$ and DMAP $(0.2 \mathrm{mg}, 0.001 \mathrm{mmol})$ was stirred at room temperature for $16 \mathrm{~h}$. The mixture was then poured into $10 \%$ aqueous $\mathrm{HCl}$ and extracted with $\mathrm{CHCl}_{3}$. The organic layer was washed with aqueous saturated $\mathrm{NaHCO}_{3}$, dried over $\mathrm{Na}_{2} \mathrm{SO}_{4}$ and concentrated under reduced pressure. The residue was then purified by silica gel (TLC Silica gel $60 \mathrm{~F}_{254}$, scraped from Merck Aluminum sheets) column chromatography $\left(\mathrm{CHCl}_{3}-\mathrm{MeOH}\right.$, 99.5:0.5) to give acetyl-trioxocane $27(14.5 \mathrm{mg}, 0.012 \mathrm{mmol}, 84 \%)$ as a colorless oil: $[\alpha]_{\mathrm{D}}+113.2(c$ 1.45, $\mathrm{CHCl}_{3}$ ); IR (film): 2929, 1742, 1456, 1365, 1109, $1044 \mathrm{~cm}^{-1} ;{ }^{1} \mathrm{H}$ NMR (500 MHz, $\left.\mathrm{CDCl}_{3}\right)$ : $\delta 2.07(\mathrm{~s}, 3 \mathrm{H}), 3.19(\mathrm{dd}, J=9.8,3.2 \mathrm{~Hz}, 2 \mathrm{H}), 3.25(\mathrm{dd}, J=9.8,3.2 \mathrm{~Hz}, 1 \mathrm{H}), 3.35(\mathrm{~s}, 3 \mathrm{H}), 3.37(\mathrm{~s}$, 6H), 3.383 (s, 3H), 3.477 (s, 3H), 3.482 (s, 3H), 3.489 (s, 3H), 3.490 (s, 3H), 3.495 (s, 3H), 3.50 (s, 3H), 3.59 (s, 3H), $3.60(\mathrm{~s}, 3 \mathrm{H}), 3.63(\mathrm{~s}, 6 \mathrm{H}), 3.70(\mathrm{~s}, 3 \mathrm{H}), 3.72(\mathrm{~s}, 3 \mathrm{H}), 4.08(\mathrm{dd}, J=11.2,3.0$ Hz, 1H), 4.18 (dd, $J=12.0,4.1 \mathrm{~Hz}, 1 \mathrm{H}), 4.38(\mathrm{dd}, J=12.0,1.6 \mathrm{~Hz}, 1 \mathrm{H}), 5.02(\mathrm{~m}, 3 \mathrm{H}), 5.03(\mathrm{~d}, J$ $=3.2 \mathrm{~Hz}, 1 \mathrm{H}), 5.06(\mathrm{~d}, J=3.2 \mathrm{~Hz}, 1 \mathrm{H}), 5.19(\mathrm{~d}, J=3.5 \mathrm{~Hz}, 1 \mathrm{H}) ;{ }^{1} \mathrm{H}$ NMR $\left(500 \mathrm{MHz}, \mathrm{C}_{6} \mathrm{D}_{6}\right): \delta$ $1.87(\mathrm{~s}, 3 \mathrm{H}), 3.26(\mathrm{~s}, 6 \mathrm{H}), 3.28(\mathrm{~s}, 3 \mathrm{H}), 3.29$ (s, 3H), $3.29(\mathrm{~s}, 3 \mathrm{H}), 3.32(\mathrm{~s}, 6 \mathrm{H}), 3.37$ (s, 3H), 3.39 (s, 3H), $3.48(\mathrm{~s}, 3 \mathrm{H}), 3.65(\mathrm{~s}, 3 \mathrm{H}), 3.68(\mathrm{~s}, 3 \mathrm{H}), 3.70(\mathrm{~s}, 3 \mathrm{H}), 3.80(\mathrm{~s}, 3 \mathrm{H}), 3.84(\mathrm{~s}, 3 \mathrm{H}), 3.85(\mathrm{~s}$, $3 \mathrm{H}), 4.06\left(\mathrm{dd}, J=10.7,10.7 \mathrm{~Hz}, 1 \mathrm{H}, \mathrm{H} 6^{\mathrm{III}}\right), 4.61\left(\mathrm{~m}, 2 \mathrm{H}, \mathrm{H}^{\mathrm{I}}\right.$ and $\left.\mathrm{H} 6^{\mathrm{I}}\right), 5.02($ br d, $J=10.7 \mathrm{~Hz}$, 1H, H6 $\left.{ }^{\mathrm{I}}\right), 5.08(\mathrm{~d}, J=3.8 \mathrm{~Hz}, 1 \mathrm{H}), 5.13(\mathrm{~d}, J=3.8 \mathrm{~Hz}, 1 \mathrm{H}), 5.17\left(\mathrm{~d}, J=3.5 \mathrm{~Hz}, 1 \mathrm{H}, \mathrm{H} 1^{\mathrm{II}}\right), 5.18(\mathrm{~d}$, $J=4.1 \mathrm{~Hz}, 1 \mathrm{H}), 5.20(\mathrm{~d}, J=3.5 \mathrm{~Hz}, 1 \mathrm{H}), 5.22\left(\mathrm{~d}, J=3.5 \mathrm{~Hz}, 1 \mathrm{H}, \mathrm{H} 1^{\mathrm{I}}\right) ;{ }^{1} \mathrm{H}$ NMR $\left(500 \mathrm{MHz}, \mathrm{C}_{6} \mathrm{D}_{6}\right.$, 1D-TOCSY, irradiation at $\left.\mathrm{H}^{\mathrm{I}}, 5.02 \mathrm{ppm}\right): \delta 3.21\left(\mathrm{dd}, J=9.6,3.4 \mathrm{~Hz}, 1 \mathrm{H}, \mathrm{H} 2^{\mathrm{I}}\right), 3.58(\mathrm{dd}, J=9.0$, $\left.9.0 \mathrm{~Hz}, 1 \mathrm{H}, \mathrm{H} 4^{\mathrm{I}}\right), 3.83\left(\mathrm{dd}, J=9.1,9.1 \mathrm{~Hz}, 1 \mathrm{H}, \mathrm{H} 3^{\mathrm{I}}\right), 4.61\left(\mathrm{~m}, 2 \mathrm{H}, \mathrm{H} 5^{\mathrm{I}}\right.$ and $\left.\mathrm{H} 6^{\mathrm{I}}\right), 5.22(\mathrm{~d}, J=2.9$ $\left.\mathrm{Hz}, 1 \mathrm{H}, \mathrm{H} 1{ }^{\mathrm{I}}\right) ;{ }^{1} \mathrm{H}$ NMR $\left(500 \mathrm{MHz}, \mathrm{C}_{6} \mathrm{D}_{6}, 1 \mathrm{D}-\mathrm{TOCSY}\right.$, irradiation at $\left.\mathrm{H} 1^{\mathrm{II}}, 5.17 \mathrm{ppm}\right): \delta 3.25(\mathrm{dd}, J$ 
$\left.=9.6,2.7 \mathrm{~Hz}, 1 \mathrm{H}, \mathrm{H} 2^{\mathrm{II}}\right), 4.15\left(\mathrm{dd}, J=9.3,9.3 \mathrm{~Hz}, 1 \mathrm{H}, \mathrm{H}{ }^{\mathrm{II}}\right), 4.26\left(\mathrm{~d}, J=9.4 \mathrm{~Hz}, 1 \mathrm{H}, \mathrm{H} 4^{\mathrm{II}}\right) ;{ }^{1} \mathrm{H}$ NMR (500 MHz, $\mathrm{CDCl}_{3}, 1 \mathrm{D}-\mathrm{ROESY}$, irradiation at $\left.\mathrm{H}^{\mathrm{I}}, 5.22 \mathrm{ppm}\right): \delta 4.26(\mathrm{~d}, J=9.2 \mathrm{~Hz}, 1 \mathrm{H}$, $\left.\mathrm{H} 4{ }^{\mathrm{II}}\right) ;{ }^{13} \mathrm{C} \mathrm{NMR}\left(125.7 \mathrm{MHz}, \mathrm{CDCl}_{3}\right): \delta 20.98\left(\mathrm{CH}_{3}\right), 57.77\left(\mathrm{CH}_{3}\right), 57.83\left(2 \times \mathrm{CH}_{3}\right), 58.12\left(\mathrm{CH}_{3}\right)$, $58.20\left(\mathrm{CH}_{3}\right), 58.39\left(\mathrm{CH}_{3}\right), 58.92\left(\mathrm{CH}_{3}\right), 58.99\left(\mathrm{CH}_{3}\right), 59.09\left(\mathrm{CH}_{3}\right), 59.17\left(\mathrm{CH}_{3}\right), 61.30\left(\mathrm{CH}_{3}\right), 61.56$ $\left(\mathrm{CH}_{3}\right), 61.89\left(\mathrm{CH}_{3}\right), 62.02\left(\mathrm{CH}_{3}\right), 62.30\left(\mathrm{CH}_{3}\right), 63.91\left(\mathrm{CH}_{2}\right), 64.07\left(\mathrm{CH}_{2}\right), 66.10(\mathrm{CH}), 69.20(\mathrm{CH})$, $70.75\left(\mathrm{CH}_{2}\right), 70.86(\mathrm{CH}), 71.21\left(\mathrm{CH}_{2}\right), 71.33\left(\mathrm{CH}_{2}\right), 71.38(\mathrm{CH}), 71.48(\mathrm{CH}), 72.02\left(\mathrm{CH}_{2}\right), 78.26$ $(\mathrm{CH}), 80.03(\mathrm{CH}), 80.60(\mathrm{CH}), 80.61(\mathrm{CH}), 80.83(\mathrm{CH}), 81.04(\mathrm{CH}), 81.06(\mathrm{CH}), 81.15(\mathrm{CH})$, $81.35(\mathrm{CH}), 81.42(\mathrm{CH}), 81.79(2 \times \mathrm{CH}), 82.09(\mathrm{CH}), 82.11(\mathrm{CH}), 82.16(\mathrm{CH}), 82.35(\mathrm{CH}), 82.50$ $(\mathrm{CH}), 82.60(\mathrm{CH}), 97.39(\mathrm{CH}), 97.46(\mathrm{CH}), 99.09(\mathrm{CH}), 99.35(\mathrm{CH}), 100.05(\mathrm{CH}), 100.71(\mathrm{CH})$, $100.75(\mathrm{C}), 170.86(\mathrm{C}) ;{ }^{13} \mathrm{C}$ NMR $\left(125.7 \mathrm{MHz}, \mathrm{C}_{6} \mathrm{D}_{6}\right): \delta 20.78\left(\mathrm{CH}_{3}\right), 57.24\left(\mathrm{CH}_{3}\right), 57.67\left(\mathrm{CH}_{3}\right)$, $57.78\left(2 \times \mathrm{CH}_{3}\right), 58.15\left(\mathrm{CH}_{3}\right), 58.25\left(\mathrm{CH}_{3}\right), 58.84\left(\mathrm{CH}_{3}\right), 58.97\left(\mathrm{CH}_{3}\right), 59.01\left(\mathrm{CH}_{3}\right), 59.10\left(\mathrm{CH}_{3}\right)$, $61.29\left(\mathrm{CH}_{3}\right), 61.56\left(\mathrm{CH}_{3}\right), 61.65\left(\mathrm{CH}_{3}\right), 61.79\left(\mathrm{CH}_{3}\right), 62.11\left(\mathrm{CH}_{3}\right), 62.14\left(\mathrm{CH}_{3}\right), 64.31\left(\mathrm{CH}_{2}\right), 64.68$ $\left(\mathrm{CH}_{2}\right), 66.70(\mathrm{CH}), 69.93(\mathrm{CH}), 71.74\left(\mathrm{CH}_{2}\right), 71.79(\mathrm{CH}), 72.23(\mathrm{CH}), 72.25\left(\mathrm{CH}_{2}\right), 72.50(\mathrm{CH})$, $72.72\left(\mathrm{CH}_{2}\right), 73.00\left(\mathrm{CH}_{2}\right), 79.05(\mathrm{CH}), 81.53(\mathrm{CH}), 81.57(\mathrm{CH}), 81.60(\mathrm{CH}), 81.63(\mathrm{CH}), 81.82$ $(\mathrm{CH}), 81.84(\mathrm{CH}), 82.04(\mathrm{CH}), 82.13(2 \times \mathrm{CH}), 82.68(\mathrm{CH}), 82.72(\mathrm{CH}), 82.96(\mathrm{CH}), 83.00(\mathrm{CH})$, $83.06(\mathrm{CH}), 83.17(\mathrm{CH}), 83.31(\mathrm{CH}), 83.36(\mathrm{CH}), 98.06(\mathrm{CH}), 98.52(\mathrm{CH}), 99.08(\mathrm{CH}), 99.18$ $(\mathrm{CH}), 99.93(\mathrm{CH}), 100.88(\mathrm{CH}), 101.20(\mathrm{C}), 170.11(\mathrm{C})$; MS (ESI $\left.{ }^{+}-\mathrm{TOF}\right): m / z(\%) 1259[(\mathrm{M}+$ $\left.\mathrm{Na})^{+}, 100\right]$; HRMS (ESI - -TOF): $m / z[\mathrm{M}+\mathrm{Na}]^{+}$calcd for $\mathrm{C}_{54} \mathrm{H}_{92} \mathrm{NaO}_{31}$ 1259.5520; found 1259.5526. Anal. calcd for $\mathrm{C}_{54} \mathrm{H}_{92} \mathrm{O}_{31}$ : C, 52.42; H, 7.49. Found: C, 52.35; H, 7.56.

Cyclo-5 ${ }^{V I}, 6^{I}$-anhydro-(5 $\left.{ }^{V I} R\right)-(2,3,6-$ tri-O-methyl- $\alpha$-D-xylo-hexos-5-ulopyranosyl)-(1 $\rightarrow 4)-2,3-$

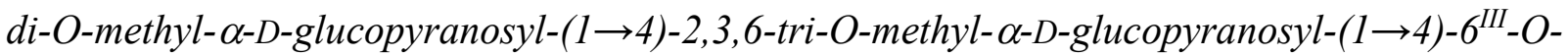

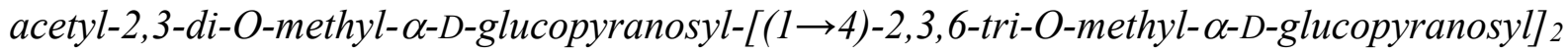
(28). A solution of alcohol 26 (24 mg, $0.02 \mathrm{mmol})$ in dry pyridine $(0.9 \mathrm{~mL})$ containing $\mathrm{Ac}_{2} \mathrm{O}(0.3$ 
$\mathrm{mL})$ and DMAP $(0.3 \mathrm{mg}, 0.002 \mathrm{mmol})$ was stirred at room temperature for $14 \mathrm{~h}$. The mixture was then poured into $10 \%$ aqueous $\mathrm{HCl}$ and extracted with $\mathrm{CHCl}_{3}$. The organic layer was washed with aqueous saturated $\mathrm{NaHCO}_{3}$, dried over $\mathrm{Na}_{2} \mathrm{SO}_{4}$ and concentrated under reduced pressure. The residue was then purified by silica gel (TLC Silica gel $60 \mathrm{~F}_{254}$, scraped from Merck Aluminum sheets) column chromatography $\left(\mathrm{CHCl}_{3}-\mathrm{MeOH}, 99.5: 0.5\right)$ to give acetyl-trioxocane 28 (10 mg, $0.008 \mathrm{mmol}$, 40\%), as a colorless oil: $[\alpha]_{\mathrm{D}}+130.8\left(c 0.98, \mathrm{CHCl}_{3}\right)$; IR (film): 2929, 1744, 1454, 1367, 1109, $1046 \mathrm{~cm}^{-1} ;{ }^{1} \mathrm{H}$ NMR (500 MHz, $\mathrm{CDCl}_{3}$ ): $\delta 2.07$ (s, 3H), $3.179(\mathrm{dd}, J=10.1,3.5 \mathrm{~Hz}, 1 \mathrm{H}), 3.198$ (dd, $J=9.8,3.5 \mathrm{~Hz}, 1 \mathrm{H}), 3.198$ (dd, $J=9.8,3.5 \mathrm{~Hz}, 1 \mathrm{H}), 3.257$ (dd, $J=9.5,3.2 \mathrm{~Hz}, 1 \mathrm{H}), 3.34$ (s, 3H), 3.37 (s, 3H), 3.381 (s, 3H), $3.384(\mathrm{~s}, 3 \mathrm{H}), 3.47$ (s, 3H), 3.476 (s, 3H), 3.489 (s, 3H), $3.494(\mathrm{~s}$, 3H), $3.50(\mathrm{~s}, 3 \mathrm{H}), 3.52(\mathrm{~s}, 3 \mathrm{H}), 3.59(\mathrm{~s}, 3 \mathrm{H}), 3.60(\mathrm{~s}, 3 \mathrm{H}), 3.638(\mathrm{~s}, 3 \mathrm{H}), 3.642(\mathrm{~s}, 3 \mathrm{H}), 3.70(\mathrm{~s}, 3 \mathrm{H})$, $3.73(\mathrm{~s}, 3 \mathrm{H}), 3.93\left(\mathrm{~d}, J=9.5 \mathrm{~Hz}, 1 \mathrm{H}, \mathrm{H} 4^{\mathrm{VII}}\right), 4.39$ (dd, $\left.J=12.1,4.3 \mathrm{~Hz}, 1 \mathrm{H}, \mathrm{H} 6^{\mathrm{II}}\right), 4.56(\mathrm{dd}, J=$ 12.3, $\left.1.9 \mathrm{~Hz}, 1 \mathrm{H}, \mathrm{H6} 6^{\mathrm{III}}\right), 4.98(\mathrm{~d}, J=3.8 \mathrm{~Hz}, 2 \mathrm{H}), 5.04(\mathrm{~d}, J=3.5 \mathrm{~Hz}, 1 \mathrm{H}), 5.05(\mathrm{~d}, J=3.5 \mathrm{~Hz}, 2 \mathrm{H})$, $5.19\left(\mathrm{~d}, J=3.2 \mathrm{~Hz}, 1 \mathrm{H}, \mathrm{H} 1^{\mathrm{I}}\right) ;{ }^{1} \mathrm{H}$ NMR $\left(500 \mathrm{MHz}, \mathrm{CDCl}_{3}, 1 \mathrm{D}-\mathrm{TOCSY}\right.$, irradiation at $\mathrm{H} 6{ }^{\mathrm{III}}, 4.56$ ppm): $\delta 3.14$ (dd, $\left.J=10.0,3.3 \mathrm{~Hz}, 1 \mathrm{H}, \mathrm{H} 2^{\mathrm{III}}\right), 3.57$ (dd, $J=9.1,9.1 \mathrm{~Hz}, 1 \mathrm{H}, \mathrm{H} 4^{\mathrm{III}}$ ), 3.69 (dd, $J=$ 9.4, 9.4 Hz, 1H, H3 $\left.{ }^{\mathrm{III}}\right), 3.77$ (m, 1H, H5 $\left.{ }^{\mathrm{III}}\right), 4.40$ (dd, $\left.J=11.7,3.9 \mathrm{~Hz}, 1 \mathrm{H}, \mathrm{H} 6^{\mathrm{III}}\right), 5.05$ (d, $J=2.5$ $\left.\mathrm{Hz}, 1 \mathrm{H}, \mathrm{H} 1^{\mathrm{III}}\right) ;{ }^{1} \mathrm{H} \mathrm{NMR}\left(500 \mathrm{MHz}, \mathrm{CDCl}_{3}, 1 \mathrm{D}-\mathrm{TOCSY}\right.$, irradiation at $\left.\mathrm{H} 1^{\mathrm{VI}}, 5.19 \mathrm{ppm}\right): \delta 3.26$ $\left(\mathrm{dd}, J=9.7,3.2 \mathrm{~Hz}, 1 \mathrm{H}, \mathrm{H} 2^{\mathrm{VI}}\right), 3.72\left(\mathrm{dd}, J=9.3,9.3 \mathrm{~Hz}, 1 \mathrm{H}, \mathrm{H} 3^{\mathrm{VI}}\right), 3.94\left(\mathrm{~d}, J=9.4 \mathrm{~Hz}, 1 \mathrm{H}, \mathrm{H} 4^{\mathrm{VI}}\right)$; ${ }^{1} \mathrm{H}$ NMR (500 MHz, $\mathrm{CDCl}_{3}, 1 \mathrm{D}-\mathrm{ROESY}$, irradiation at $\left.\mathrm{H}^{\mathrm{VI}}{ }^{\mathrm{VI}}, 3.93 \mathrm{ppm}\right): \delta 5.04(\mathrm{~d}, J=3.2 \mathrm{~Hz}, 1 \mathrm{H}$, $\left.\mathrm{H} 1{ }^{\mathrm{V}}\right) ;{ }^{13} \mathrm{C} \mathrm{NMR}\left(125.7 \mathrm{MHz}, \mathrm{CDCl}_{3}\right): \delta 20.90\left(\mathrm{CH}_{3}\right), 57.69\left(\mathrm{CH}_{3}\right), 57.82\left(\mathrm{CH}_{3}\right), 58.11\left(2 \times \mathrm{CH}_{3}\right)$, $58.28\left(\mathrm{CH}_{3}\right), 58.46\left(\mathrm{CH}_{3}\right), 58.83\left(\mathrm{CH}_{3}\right), 58.93\left(\mathrm{CH}_{3}\right), 59.05\left(\mathrm{CH}_{3}\right), 59.10\left(\mathrm{CH}_{3}\right), 61.29\left(\mathrm{CH}_{3}\right), 61.48$ $\left(\mathrm{CH}_{3}\right), 61.59\left(\mathrm{CH}_{3}\right), 61.85\left(\mathrm{CH}_{3}\right), 62.08\left(\mathrm{CH}_{3}\right), 62.27\left(\mathrm{CH}_{3}\right), 63.69\left(\mathrm{CH}_{2}\right), 63.85\left(\mathrm{CH}_{2}\right), 66.21(\mathrm{CH})$, $69.89(\mathrm{CH}), 70.77\left(\mathrm{CH}_{2}\right), 70.86(\mathrm{CH}), 70.98(\mathrm{CH}), 71.26(\mathrm{CH}), 71.35\left(\mathrm{CH}_{2}\right), 71.56\left(\mathrm{CH}_{2}\right), 71.96$ $\left(\mathrm{CH}_{2}\right), 78.29(\mathrm{CH}), 79.87(\mathrm{CH}), 80.61(\mathrm{CH}), 80.76(2 \times \mathrm{CH}), 81.04(\mathrm{CH}), 81.09(\mathrm{CH}), 81.17(\mathrm{CH})$, 
$81.39(\mathrm{CH}), 81.51(\mathrm{CH}), 81.75(\mathrm{CH}), 81.94(\mathrm{CH}), 82.10(\mathrm{CH}), 82.17(\mathrm{CH}), 82.38(\mathrm{CH}), 82.49$ $(\mathrm{CH}), 82.71(2 \times \mathrm{CH}), 97.32(\mathrm{CH}), 97.37(\mathrm{CH}), 99.50(\mathrm{CH}), 99.61(\mathrm{CH}), 99.78(\mathrm{CH}), 100.17(\mathrm{CH})$,

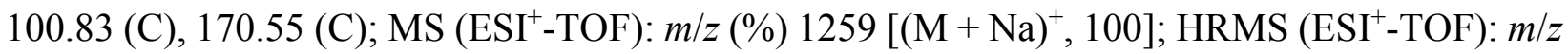
$[\mathrm{M}+\mathrm{Na}]^{+}$calcd for $\mathrm{C}_{54} \mathrm{H}_{92} \mathrm{NaO}_{31}$ 1259.5520; found 1259.5504. Anal. calcd for $\mathrm{C}_{54} \mathrm{H}_{92} \mathrm{O}_{31}: \mathrm{C}$, 52.42; H, 7.49. Found: C, 52.20; H, 7.65.

Oxidative HAT of $2^{\text {I-VII }} 3^{I-V I I}, 6^{I I I-V I I}$-nonadeca-O-methyl- $\beta$-cyclomaltoheptaose (29). A solution of alcohol $29^{17 \mathrm{a}}(160 \mathrm{mg}, 0.114 \mathrm{mmol})$ in dry $\mathrm{CH}_{2} \mathrm{Cl}_{2}(4.6 \mathrm{~mL})$ containing DIB (110 mg, 0.34 $\mathrm{mmol})$ and $\mathrm{I}_{2}(49 \mathrm{mg}, 0.19 \mathrm{mmol})$ was stirred under nitrogen at $28{ }^{\circ} \mathrm{C}$ for $2.5 \mathrm{~h}$ while irradiated with two $80 \mathrm{~W}$ tungsten-filament lamps. The reaction mixture was then directly loaded onto a silica gel (TLC Silica gel $60 \mathrm{~F}_{254}$, scraped from Merck Aluminum sheets) column chromatography (hexanes-acetone, $65: 35 \rightarrow 55: 45)$ to give cyclo- ${ }^{\mathrm{VII}}, 6^{\mathrm{I}}$-anhydro-( $\left.5^{\mathrm{VII}} R\right)-(2,3,6$-tri- $O$-methyl- $\alpha$-Dxylo-hexos-5-ulopyranosyl)-( $(1 \rightarrow 4)-\left(1^{\mathrm{I}} R\right)-4^{\mathrm{II}}, 6^{\mathrm{II}}$-O-(2,3-di- $O$-methyl-D-glucopyranosylidene)-2,3-

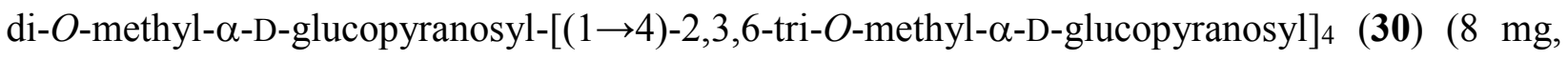
$0.0057 \mathrm{mmol}, 5 \%)$, ten-membered lactone $31(17.1 \mathrm{mg}, 0.012 \mathrm{mmol}, 11 \%)$, and a mixture (54.1 $\mathrm{mg}$ ) of two alcohols $\mathbf{3 2}$ and $\mathbf{3 3}$ that was separated after acetylation as mentioned below. To a solution of this mixture in anhydrous pyridine $(3 \mathrm{~mL})$ were added $\mathrm{Ac}_{2} \mathrm{O}(1 \mathrm{~mL})$ and DMAP $(1 \mathrm{mg})$. After $14 \mathrm{~h}$ at room temperature, the solution was poured into ice-water and extracted with $\mathrm{CH}_{2} \mathrm{Cl}_{2}$. The organic phase was successively washed with aqueous solutions of $\mathrm{HCl}(10 \%)$ and saturated $\mathrm{NaHCO}_{3}$, dried over $\mathrm{Na}_{2} \mathrm{SO}_{4}$, and concentrated under reduced pressure. The residue $(86.5 \mathrm{mg}$ ) was purified by silica gel (TLC Silica gel $60 \mathrm{~F}_{254}$, scraped from Merck Aluminum sheets) column chromatography $\left(\mathrm{CHCl}_{3}-\mathrm{MeOH}, 99: 1\right)$ to give cyclo- $5^{\mathrm{VII}}, 6^{\mathrm{I}}$-anhydro-( $\left.5^{\mathrm{VII}} R\right)-(2,3,6$-tri- $O$-methyl- $\alpha-$ D-xylo-hexos-5-ulopyranosyl)-(1 $\rightarrow 4)-2,3$-di- $O$-methyl- $\alpha$-D-glucopyranosyl-( $(1 \rightarrow 4)-6^{\mathrm{II}}$ - $O$-acetyl-

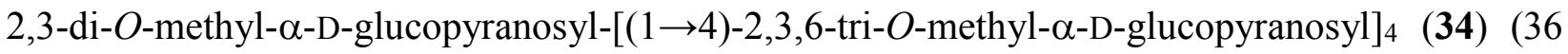


$\mathrm{mg}, 0.025 \mathrm{mmol}, 22 \%)$ and the lactone 35 (16 mg, $0.013 \mathrm{mmol}, 11 \%)$. Compound 30: colorless oil, $[\alpha]_{\mathrm{D}}+108.0$ (c 0.45, $\left.\mathrm{CHCl}_{3}\right) ; \mathrm{IR}$ (film): 2925, 1454, 1369, 1143, 1091, $1042 \mathrm{~cm}^{-1} ;{ }^{1} \mathrm{H}$ NMR (500 $\left.\mathrm{MHz}_{\mathrm{CDCl}}\right): \delta 3.06(\mathrm{~d}, J=9.5 \mathrm{~Hz}, 1 \mathrm{H}), 3.17(\mathrm{dd}, J=9.5,3.7 \mathrm{~Hz}, 1 \mathrm{H}), 3.19(\mathrm{dd}, J=9.5,3.2 \mathrm{~Hz}$, 1H), $3.209(\mathrm{dd}, J=9.2,4.1 \mathrm{~Hz}, 1 \mathrm{H}), 3.211(\mathrm{dd}, J=9.5,3.5 \mathrm{~Hz}, 1 \mathrm{H}), 3.22(\mathrm{dd}, J=9.5,3.8 \mathrm{~Hz}, 1 \mathrm{H})$, $3.27(\mathrm{dd}, J=9.8,3.8 \mathrm{~Hz}, 1 \mathrm{H}), 3.33(\mathrm{~s}, 3 \mathrm{H}), 3.35(\mathrm{~s}, 3 \mathrm{H}), 3.37(\mathrm{~s}, 3 \mathrm{H}), 3.39(\mathrm{~s}, 3 \mathrm{H}), 3.39(\mathrm{~s}, 3 \mathrm{H})$, $3.47(\mathrm{~s}, 3 \mathrm{H}), 3.47(\mathrm{~s}, 3 \mathrm{H}), 3.50(\mathrm{~s}, 3 \mathrm{H}), 3.51(\mathrm{~s}, 3 \mathrm{H}), 3.55(\mathrm{~s}, 3 \mathrm{H}), 3.56(\mathrm{~s}, 3 \mathrm{H}), 3.59(\mathrm{~s}, 9 \mathrm{H}), 3.64$ $(\mathrm{s}, 3 \mathrm{H}), 3.64(\mathrm{~s}, 3 \mathrm{H}), 3.66(\mathrm{~s}, 3 \mathrm{H}), 3.70(\mathrm{~s}, 3 \mathrm{H}), 3.71(\mathrm{~s}, 3 \mathrm{H}), 4.22(\mathrm{dd}, J=10.4,8.2 \mathrm{~Hz}, 1 \mathrm{H}), 5.04$ $(\mathrm{d}, J=3.5 \mathrm{~Hz}, 1 \mathrm{H}), 5.07(\mathrm{~d}, J=3.2 \mathrm{~Hz}, 2 \mathrm{H}), 5.20(\mathrm{~d}, J=3.8 \mathrm{~Hz}, 1 \mathrm{H}), 5.23(\mathrm{~d}, J=4.4 \mathrm{~Hz}, 1 \mathrm{H})$, $5.34(\mathrm{~d}, J=4.1 \mathrm{~Hz}, 1 \mathrm{H}) ;{ }^{1} \mathrm{H} \mathrm{NMR}\left(500 \mathrm{MHz}, \mathrm{CDCl}_{3}, 1 \mathrm{D}-\mathrm{TOCSY}\right.$, irradiation at $\left.\mathrm{H} 2{ }^{\mathrm{I}}, 3.06 \mathrm{ppm}\right)$ : $\delta 3.40\left(\mathrm{dd}, J=9.7,9.7 \mathrm{~Hz}, 1 \mathrm{H}, \mathrm{H} 3^{\mathrm{I}}\right), 3.54\left(\mathrm{dd}, J=9.3,9.3 \mathrm{~Hz}, 1 \mathrm{H}, \mathrm{H} 4^{\mathrm{I}}\right), 3.75\left(\mathrm{~m}, 2 \mathrm{H}, \mathrm{H} 6^{\mathrm{I}}\right.$ and $\mathrm{H}^{\mathrm{I}}$ ), $3.90\left(\mathrm{dd}, J=9.9,9.9 \mathrm{~Hz}, 1 \mathrm{H}, \mathrm{H6}^{\mathrm{I}}\right) ;{ }^{1} \mathrm{H} \mathrm{NMR}\left(500 \mathrm{MHz}, \mathrm{CDCl}_{3}, 1 \mathrm{D}-\mathrm{TOCSY}\right.$, irradiation at $\left.\mathrm{H}^{\mathrm{II}}, 4.22 \mathrm{ppm}\right): \delta 3.21\left(\mathrm{dd}, J=9.6,4.3 \mathrm{~Hz}, 1 \mathrm{H}, \mathrm{H} 2^{\mathrm{II}}\right), 3.57\left(\mathrm{dd}, J=9.2,9.2 \mathrm{~Hz}, 1 \mathrm{H}, \mathrm{H} 3^{\mathrm{II}}\right), 3.63$ $\left(\mathrm{dd}, J=10.3,6.8 \mathrm{~Hz}, 1 \mathrm{H}, \mathrm{H} 6^{\mathrm{II}}\right), 3.72\left(\mathrm{dd}, J=10.0,10.0 \mathrm{~Hz}, 1 \mathrm{H}, \mathrm{H} 4^{\mathrm{II}}\right), 4.00\left(\mathrm{~m}, 1 \mathrm{H}, \mathrm{H} 5^{\mathrm{II}}\right), 5.23(\mathrm{~d}$, $\left.J=4.0 \mathrm{~Hz}, 1 \mathrm{H}, \mathrm{H} 1{ }^{\mathrm{II}}\right) ;{ }^{13} \mathrm{C} \mathrm{NMR}\left(125.7 \mathrm{MHz}, \mathrm{CDCl}_{3}\right): \delta 57.80\left(\mathrm{CH}_{3}\right), 57.88\left(\mathrm{CH}_{3}\right), 58.07\left(\mathrm{CH}_{3}\right)$, $58.22\left(\mathrm{CH}_{3}\right), 58.91\left(\mathrm{CH}_{3}\right), 59.02\left(2 \times \mathrm{CH}_{3}\right), 59.04\left(\mathrm{CH}_{3}\right), 59.14\left(\mathrm{CH}_{3}\right), 59.44\left(\mathrm{CH}_{3}\right), 60.04\left(\mathrm{CH}_{3}\right)$, $60.57\left(\mathrm{CH}_{3}\right), 60.70\left(\mathrm{CH}_{3}\right), 60.96\left(\mathrm{CH}_{3}\right), 61.07\left(\mathrm{CH}_{3}\right), 61.18\left(\mathrm{CH}_{3}\right), 61.67\left(\mathrm{CH}_{3}\right), 61.70\left(\mathrm{CH}_{3}\right), 61.84$ $\left(\mathrm{CH}_{2}\right), 62.04\left(\mathrm{CH}_{3}\right), 63.66(\mathrm{CH}), 64.36\left(\mathrm{CH}_{2}\right), 67.62(\mathrm{CH}), 70.05(\mathrm{CH}), 70.07(\mathrm{CH}), 71.06(\mathrm{CH})$, $71.09\left(\mathrm{CH}_{2}\right), 71.41(\mathrm{CH}), 71.49\left(\mathrm{CH}_{2}\right), 71.83\left(\mathrm{CH}_{2}\right), 71.84\left(\mathrm{CH}_{2}\right), 71.93\left(\mathrm{CH}_{2}\right), 74.10(\mathrm{CH}), 77.50$ $(\mathrm{CH}), 77.87(\mathrm{CH}), 78.37(\mathrm{CH}), 79.20(\mathrm{CH}), 80.18(\mathrm{CH}), 80.35(\mathrm{CH}), 81.02(\mathrm{CH}), 81.05(\mathrm{CH})$, $81.11(\mathrm{CH}), 81.37(\mathrm{CH}), 81.70(\mathrm{CH}), 81.80(\mathrm{CH}), 81.88(\mathrm{CH}), 81.92(\mathrm{CH}), 82.28(\mathrm{CH}), 82.34$ $(\mathrm{CH}), 82.45(\mathrm{CH}), 82.48(\mathrm{CH}), 82.76(\mathrm{CH}), 84.92(\mathrm{CH}), 97.04(\mathrm{CH}), 97.99(\mathrm{CH}), 98.61(\mathrm{CH})$, $98.83(\mathrm{CH}), 99.81(\mathrm{CH}), 100.31(\mathrm{CH}), 101.04(\mathrm{C}), 111.58(\mathrm{C})$; MS (ESI $\left.{ }^{+} \mathrm{TOF}\right): \mathrm{m} / z(\%) 1419$ [(M $\left.+\mathrm{Na})^{+}, 100\right]$; HRMS (ESI $\left.-\mathrm{TOF}\right): m / z[\mathrm{M}+\mathrm{Na}]^{+}$calcd for $\mathrm{C}_{61} \mathrm{H}_{104} \mathrm{NaO}_{35}$ 1419.6256; found 
1419.6281. Anal. calcd for $\mathrm{C}_{61} \mathrm{H}_{104} \mathrm{O}_{35}: \mathrm{C}, 52.43 ; \mathrm{H}, 7.50$. Found: $\mathrm{C}, 52.35 ; \mathrm{H}, 7.73$. Compound 31: colorless oil, $[\alpha]_{\mathrm{D}}+121.8\left(c 0.68, \mathrm{CHCl}_{3}\right)$; IR (film): 2929, 1755, 1745, 1454, 1367, 1161, 1106, $1039 \mathrm{~cm}^{-1} ;{ }^{1} \mathrm{H}$ NMR (500 MHz, $\left.\mathrm{CDCl}_{3}\right): \delta 2.14(\mathrm{~s}, 3 \mathrm{H}), 3.04(\mathrm{dd}, J=9.8,3.5 \mathrm{~Hz}, 1 \mathrm{H}), 3.16$ (dd, $J=9.8,3.5 \mathrm{~Hz}, 1 \mathrm{H}), 3.16$ (dd, $J=9.8,3.5 \mathrm{~Hz}, 1 \mathrm{H}), 3.18$ (dd, $J=9.8,3.2 \mathrm{~Hz}, 1 \mathrm{H}), 3.19$ (dd, $J$ $=9.8,3.5 \mathrm{~Hz}, 1 \mathrm{H}), 3.24(\mathrm{dd}, J=9.5,3.8 \mathrm{~Hz}, 1 \mathrm{H}), 3.35(\mathrm{~s}, 3 \mathrm{H}), 3.361(\mathrm{~s}, 3 \mathrm{H}), 3.365(\mathrm{~s}, 3 \mathrm{H}), 3.37$ (s, 3H), $3.38(\mathrm{~s}, 3 \mathrm{H}), 3.46(\mathrm{~s}, 6 \mathrm{H}), 3.485(\mathrm{~s}, 3 \mathrm{H}), 3.49$ (s, 3H), 3.51 (s, 3H), $3.53(\mathrm{~s}, 3 \mathrm{H}), 3.58(\mathrm{~s}$, 3H), $3.59(\mathrm{~s}, 3 \mathrm{H}), 3.61(\mathrm{~s}, 3 \mathrm{H}), 3.62(\mathrm{~s}, 3 \mathrm{H}), 3.63(\mathrm{~s}, 3 \mathrm{H}), 3.66(\mathrm{~s}, 3 \mathrm{H}), 3.69(\mathrm{~s}, 3 \mathrm{H}), 3.73(\mathrm{~s}, 3 \mathrm{H})$, $4.36(\mathrm{ddd}, J=9.9,9.9,5.8 \mathrm{~Hz}, 1 \mathrm{H}), 4.58(\mathrm{dd}, J=10.7,5.7 \mathrm{~Hz}, 1 \mathrm{H}), 4.96(\mathrm{~d}, J=3.8 \mathrm{~Hz}, 1 \mathrm{H}), 5.00$ $(\mathrm{d}, J=3.5 \mathrm{~Hz}, 1 \mathrm{H}), 5.09(\mathrm{~d}, J=3.8 \mathrm{~Hz}, 2 \mathrm{H}), 5.17(\mathrm{~d}, J=3.5 \mathrm{~Hz}, 1 \mathrm{H}), 5.26(\mathrm{~d}, J=3.8 \mathrm{~Hz}, 1 \mathrm{H})$, $6.30(\mathrm{~d}, J=1.9 \mathrm{~Hz}, 1 \mathrm{H}) ;{ }^{1} \mathrm{H}$ NMR $\left(500 \mathrm{MHz}, \mathrm{CDCl}_{3}, 1 \mathrm{D}-\mathrm{TOCSY}\right.$, irradiation at $\left.\mathrm{H} 5^{\mathrm{II}}, 4.36 \mathrm{ppm}\right)$ : $\delta 3.05\left(\mathrm{dd}, J=9.9,3.4 \mathrm{~Hz}, 1 \mathrm{H}, \mathrm{H} 2^{\mathrm{II}}\right), 3.45\left(\mathrm{dd}, J=8.8,8.8 \mathrm{~Hz}, 1 \mathrm{H}, \mathrm{H} 3^{\mathrm{II}}\right), 3.55(\mathrm{dd}, J=9.3,9.3 \mathrm{~Hz}$, $\left.1 \mathrm{H}, \mathrm{H} 4^{\mathrm{II}}\right), 3.81\left(\mathrm{dd}, J=10.4,10.4 \mathrm{~Hz}, 1 \mathrm{H}, \mathrm{H} 6^{\mathrm{II}}\right), 4.59\left(\mathrm{dd}, J=10.8,5.7 \mathrm{~Hz}, 1 \mathrm{H}, \mathrm{H} 6^{\mathrm{II}}\right), 5.00(\mathrm{~d}, J=$ $2.9 \mathrm{~Hz}, 1 \mathrm{H}, \mathrm{H} 1^{\mathrm{II}}$ ); ${ }^{1} \mathrm{H}$ NMR (500 MHz, $\mathrm{CDCl}_{3}, 1 \mathrm{D}-\mathrm{TOCSY}$, irradiation at $\left.\mathrm{H} 1^{\mathrm{I}}, 6.30 \mathrm{ppm}\right): \delta 3.34$ $\left(\mathrm{d}, J=9.0 \mathrm{~Hz}, 1 \mathrm{H}, \mathrm{H} 2^{\mathrm{I}}\right), 3.60\left(\mathrm{~m}, 1 \mathrm{H}, \mathrm{H} 3^{\mathrm{I}}\right), 3.90\left(\mathrm{~d}, J=9.4 \mathrm{~Hz}, 1 \mathrm{H}, \mathrm{H} 4^{\mathrm{I}}\right) ;{ }^{1} \mathrm{H}$ NMR $(500 \mathrm{MHz}$, $\mathrm{CDCl}_{3}, 1 \mathrm{D}-\mathrm{ROESY}$, irradiation at $\left.\mathrm{H}^{\mathrm{I}}, 6.30 \mathrm{ppm}\right): \delta 3.34\left(\mathrm{~d}, J=7.1 \mathrm{~Hz}, 1 \mathrm{H}, \mathrm{H} 2^{\mathrm{I}}\right), 3.55(\mathrm{dd}, J=$ 9.3, $\left.9.3 \mathrm{~Hz}, 1 \mathrm{H}, \mathrm{H}^{\mathrm{II}}\right), 3.90\left(\mathrm{~d}, J=9.4 \mathrm{~Hz}, 1 \mathrm{H}, \mathrm{H} 4^{\mathrm{I}}\right) ;{ }^{13} \mathrm{C} \mathrm{NMR}\left(125.7 \mathrm{MHz}, \mathrm{CDCl}_{3}\right): \delta 21.43\left(\mathrm{CH}_{3}\right)$, $57.86\left(\mathrm{CH}_{3}\right), 58.29\left(2 \times \mathrm{CH}_{3}\right), 58.39\left(\mathrm{CH}_{3}\right), 58.40\left(\mathrm{CH}_{3}\right), 58.83\left(\mathrm{CH}_{3}\right), 58.94\left(\mathrm{CH}_{3}\right), 58.99\left(\mathrm{CH}_{3}\right)$, $59.01\left(\mathrm{CH}_{3}\right), 59.04\left(2 \times \mathrm{CH}_{3}\right), 60.97\left(\mathrm{CH}_{3}\right), 61.25\left(\mathrm{CH}_{3}\right), 61.50\left(\mathrm{CH}_{3}\right), 61.69\left(2 \times \mathrm{CH}_{3}\right), 61.73$ $\left(\mathrm{CH}_{3}\right), 62.20\left(2 \times \mathrm{CH}_{3}\right), 63.34(\mathrm{CH}), 65.84\left(\mathrm{CH}_{2}\right), 70.17(\mathrm{CH}), 70.69(\mathrm{CH}), 70.82\left(\mathrm{CH}_{2}\right), 70.93(2$ $\times \mathrm{CH}), 71.09(\mathrm{CH}), 71.12\left(\mathrm{CH}_{2}\right), 71.14\left(\mathrm{CH}_{2}\right), 71.35\left(\mathrm{CH}_{2}\right), 71.66\left(\mathrm{CH}_{2}\right), 78.64(\mathrm{CH}), 79.61(\mathrm{CH})$, $80.14(\mathrm{CH}), 80.52(\mathrm{CH}), 80.64(\mathrm{CH}), 80.89(\mathrm{CH}), 81.32(2 \times \mathrm{CH}), 81.42(\mathrm{CH}), 81.58(\mathrm{CH}), 81.64$ $(\mathrm{CH}), 81.68(\mathrm{CH}), 81.81(2 \times \mathrm{CH}), 81.84(\mathrm{CH}), 82.01(\mathrm{CH}), 82.05(\mathrm{CH}), 82.13(\mathrm{CH}), 82.27(\mathrm{CH})$, $83.42(\mathrm{CH}), 84.59(\mathrm{CH}), 94.21(\mathrm{CH}), 97.86(\mathrm{CH}), 98.70(\mathrm{CH}), 98.83(\mathrm{CH}), 99.11(\mathrm{CH}), 99.38$ 
(CH), $99.98(\mathrm{CH}), 170.81(\mathrm{C}), 170.98(\mathrm{C})$; $\mathrm{MS}\left(\mathrm{ESI}^{+}-\mathrm{TOF}\right): m / z(\%) 1449\left[(\mathrm{M}+\mathrm{Na})^{+}, 100\right]$; HRMS (ESI ${ }^{+}$-TOF): $m / z[\mathrm{M}+\mathrm{Na}]^{+}$calcd for $\mathrm{C}_{62} \mathrm{H}_{106} \mathrm{NaO}_{36}$ 1449.6362; found 1449.6360. Anal. calcd for $\mathrm{C}_{62} \mathrm{H}_{106} \mathrm{O}_{36}: \mathrm{C}, 52.17 ; \mathrm{H}, 7.48$. Found: $\mathrm{C}, 51.91 ; \mathrm{H}, 7.48$. Compound 34: colorless oil, $[\alpha]_{D}+105.2$ (c 0.94, $\mathrm{CHCl}_{3}$ ); IR (film): 2933, 1745, 1454, 1369, 1161, 1109, $1041 \mathrm{~cm}^{-1}$; ${ }^{1} \mathrm{H}$ NMR (500 MHz, $\left.\mathrm{CDCl}_{3}\right): \delta 2.08(\mathrm{~s}, 3 \mathrm{H}), 3.13(\mathrm{dd}, J=9.5,3.5 \mathrm{~Hz}, 1 \mathrm{H}), 3.28(\mathrm{dd}, J=9.6,3.6 \mathrm{~Hz}, 1 \mathrm{H}), 3.368(\mathrm{~s}, 3 \mathrm{H})$, $3.373(\mathrm{~s}, 3 \mathrm{H}), 3.38(\mathrm{~s}, 6 \mathrm{H}), 3.40(\mathrm{~s}, 3 \mathrm{H}), 3.46(\mathrm{~s}, 6 \mathrm{H}), 3.50(\mathrm{~s}, 9 \mathrm{H}), 3.51(\mathrm{~s}, 6 \mathrm{H}), 3.58(\mathrm{~s}, 3 \mathrm{H}), 3.61$ (s, 3H), $3.62(\mathrm{~s}, 3 \mathrm{H}), 3.62(\mathrm{~s}, 3 \mathrm{H}), 3.64(\mathrm{~s}, 3 \mathrm{H}), 3.65(\mathrm{~s}, 3 \mathrm{H}), 3.69$ (s, 3H), $4.29(\mathrm{dd}, J=12.3,3.8$ Hz, 1H), 4.49 (dd, $J=12.0,1.9 \mathrm{~Hz}, 1 \mathrm{H}), 4.92$ (d, $J=3.5 \mathrm{~Hz}, 1 \mathrm{H}), 5.07$ (d, $J=3.5 \mathrm{~Hz}, 1 \mathrm{H}), 5.074$ $(\mathrm{d}, J=3.8 \mathrm{~Hz}, 1 \mathrm{H}), 5.09(\mathrm{~d}, J=3.5 \mathrm{~Hz}, 1 \mathrm{H}), 5.13(\mathrm{~d}, J=3.5 \mathrm{~Hz}, 1 \mathrm{H}), 5.20(\mathrm{~d}, J=3.8 \mathrm{~Hz}, 1 \mathrm{H})$, $5.23(\mathrm{~d}, J=3.8 \mathrm{~Hz}, 1 \mathrm{H}) ;{ }^{1} \mathrm{H} \mathrm{NMR}\left(500 \mathrm{MHz}, \mathrm{CDCl}_{3}, 1 \mathrm{D}-\mathrm{TOCSY}\right.$, irradiation at $\left.\mathrm{H} 1^{\mathrm{I}}, 4.92 \mathrm{ppm}\right)$ : $\delta 3.14\left(\mathrm{dd}, J=9.0,2.8 \mathrm{~Hz}, 1 \mathrm{H}, \mathrm{H} 2^{\mathrm{I}}\right), 3.53\left(\mathrm{dd}, J=8.7,8.7 \mathrm{~Hz}, 1 \mathrm{H}, \mathrm{H} 4^{\mathrm{I}}\right), 3.59(\mathrm{dd}, J=9.5,9.5 \mathrm{~Hz}$, $\left.1 \mathrm{H}, \mathrm{H} 3{ }^{\mathrm{I}}\right) ;{ }^{1} \mathrm{H}$ NMR (500 MHz, $\mathrm{CDCl}_{3}, 1 \mathrm{D}-\mathrm{TOCSY}$, irradiation at $\left.\mathrm{H} 6{ }^{\mathrm{II}}, 4.29 \mathrm{ppm}\right): \delta 3.19$ (dd, $J=$ 9.7, 3.9 Hz, 1H, H2 $\left.2^{\mathrm{II}}\right), 3.40\left(\mathrm{dd}, J=9.2,9.2 \mathrm{~Hz}, 1 \mathrm{H}, \mathrm{H} 3^{\mathrm{II}}\right), 3.57\left(\mathrm{dd}, J=9.7,9.7 \mathrm{~Hz}, 1 \mathrm{H}, \mathrm{H} 4^{\mathrm{II}}\right), 4.49$ (br d, $\left.J=11.6 \mathrm{~Hz}, 1 \mathrm{H}, \mathrm{H} 6^{\mathrm{II}}\right), 5.08\left(\mathrm{~d}, J=3.3 \mathrm{~Hz}, 1 \mathrm{H}, \mathrm{H1}{ }^{\mathrm{II}}\right) ;{ }^{1} \mathrm{H}$ NMR $\left(500 \mathrm{MHz}, \mathrm{CDCl}_{3}, 1 \mathrm{D}-\right.$ ROESY, irradiation at $\left.\mathrm{H} 1^{\mathrm{I}}, 4.92 \mathrm{ppm}\right): \delta 3.14\left(\mathrm{dd}, J=9.4,3.2 \mathrm{~Hz}, 1 \mathrm{H}, \mathrm{H} 2^{\mathrm{I}}\right), 3.56(\mathrm{dd}, J=9.4,9.4$ $\left.\mathrm{Hz}, 1 \mathrm{H}, \mathrm{H} 4{ }^{\mathrm{II}}\right) ;{ }^{13} \mathrm{C} \mathrm{NMR}\left(125.7 \mathrm{MHz}, \mathrm{CDCl}_{3}\right): \delta 20.84\left(\mathrm{CH}_{3}\right), 57.86\left(\mathrm{CH}_{3}\right), 57.89\left(\mathrm{CH}_{3}\right), 58.09$ $\left(\mathrm{CH}_{3}\right), 58.42\left(\mathrm{CH}_{3}\right), 58.45\left(\mathrm{CH}_{3}\right), 58.72\left(\mathrm{CH}_{3}\right), 58.88\left(\mathrm{CH}_{3}\right), 58.97\left(\mathrm{CH}_{3}\right), 59.00\left(2 \times \mathrm{CH}_{3}\right), 59.07$ $\left(\mathrm{CH}_{3}\right), 59.11\left(\mathrm{CH}_{3}\right), 60.81\left(\mathrm{CH}_{3}\right), 61.02\left(\mathrm{CH}_{3}\right), 61.35\left(\mathrm{CH}_{3}\right), 61.55\left(\mathrm{CH}_{3}\right), 61.66\left(\mathrm{CH}_{3}\right), 61.85$ $\left(\mathrm{CH}_{3}\right), 62.16\left(\mathrm{CH}_{3}\right), 63.36\left(\mathrm{CH}_{2}\right), 64.58\left(\mathrm{CH}_{2}\right), 67.04(\mathrm{CH}), 69.46(\mathrm{CH}), 70.67(\mathrm{CH}), 70.89(\mathrm{CH})$, $70.96(\mathrm{CH}), 70.96\left(\mathrm{CH}_{2}\right), 70.99(\mathrm{CH}), 71.03\left(\mathrm{CH}_{2}\right), 71.36\left(\mathrm{CH}_{2}\right), 71.74\left(\mathrm{CH}_{2}\right), 71.83\left(\mathrm{CH}_{2}\right), 77.62$ $(\mathrm{CH}), 78.85(\mathrm{CH}), 79.05(\mathrm{CH}), 80.19(\mathrm{CH}), 80.29(\mathrm{CH}), 80.51(\mathrm{CH}), 80.82(\mathrm{CH}), 81.13(\mathrm{CH})$, $81.21(\mathrm{CH}), 81.31(\mathrm{CH}), 81.42(\mathrm{CH}), 81.46(\mathrm{CH}), 81.51(\mathrm{CH}), 81.79(2 \times \mathrm{CH}), 81.80(\mathrm{CH}), 81.83$ $(\mathrm{CH}), 81.98(\mathrm{CH}), 82.27(\mathrm{CH}), 82.35(\mathrm{CH}), 82.67(\mathrm{CH}), 97.02(\mathrm{CH}), 97.81(\mathrm{CH}), 98.95(\mathrm{CH})$, 
$99.02(\mathrm{CH}), 99.03(\mathrm{CH}), 99.39(\mathrm{CH}), 99.49(\mathrm{CH}), 101.12(\mathrm{C}), 170.50(\mathrm{C})$; MS (ESI $-\mathrm{TOF}): \mathrm{m} / \mathrm{z}$ (\%) $1463\left[(\mathrm{M}+\mathrm{Na})^{+}, 100\right]$; HRMS (ESI $\left.-\mathrm{TOF}\right): \mathrm{m} / z[\mathrm{M}+\mathrm{Na}]^{+}$calcd for $\mathrm{C}_{63} \mathrm{H}_{108} \mathrm{NaO}_{36} 1463.6518$; found 1463.6530. Anal. calcd for $\mathrm{C}_{63} \mathrm{H}_{108} \mathrm{O}_{36}$ : C, 52.49; H, 7.55. Found: $\mathrm{C}, 52.58 ; \mathrm{H}, 7.63$. Compound 35: colorless oil, $[\alpha]_{\mathrm{D}}+120.3$ ( $c$ 0.64, $\mathrm{CHCl}_{3}$ ); IR (film): 2929, 1745, 1454, 1369, 1159, 1107, $1041 \mathrm{~cm}^{-1} ;{ }^{1} \mathrm{H}$ NMR (500 MHz, $\left.\mathrm{CDCl}_{3}\right): \delta 2.10(\mathrm{~s}, 3 \mathrm{H}), 3.18(\mathrm{dd}, J=9.8,3.8 \mathrm{~Hz}, 4 \mathrm{H}), 3.25$ (dd, $J=9.8,3.8 \mathrm{~Hz}, 1 \mathrm{H}), 3.35$ (s, 3H), 3.37 (s, 3H), 3.38 (s, 3H), 3.385 (s, 3H), 3.389 (s, 3H), 3.45 (dd, $J=9.8,2.8 \mathrm{~Hz}, 1 \mathrm{H}), 3.49$ (s, 3H), 3.49 (s, 9H), 3.51 (s, 3H), $3.54(\mathrm{~s}, 3 \mathrm{H}), 3.60(\mathrm{~s}, 3 \mathrm{H}), 3.62$ (s, 9H), 3.63 (s, 3H), 3.64 (s, 3H), 3.67 (s, 6H), 3.95 (dd, $J=9.5,9.5 \mathrm{~Hz}, 1 \mathrm{H}), 3.99$ (ddd, $J=9.8$, 3.9, $1.7 \mathrm{~Hz}, 1 \mathrm{H}), 4.17(\mathrm{~d}, J=8.5 \mathrm{~Hz}, 1 \mathrm{H}), 4.21(\mathrm{ddd}, J=10.1,1.9,1.9 \mathrm{~Hz}, 1 \mathrm{H}), 4.29(\mathrm{dd}, J=12.5$, $3.9 \mathrm{~Hz}, 1 \mathrm{H}), 4.45(\mathrm{dd}, J=12.3,1.9 \mathrm{~Hz}, 1 \mathrm{H}), 5.11(\mathrm{~d}, J=3.5 \mathrm{~Hz}, 1 \mathrm{H}), 5.12(\mathrm{~d}, J=3.8 \mathrm{~Hz}, 1 \mathrm{H})$, $5.13(\mathrm{~d}, J=3.8 \mathrm{~Hz}, 1 \mathrm{H}), 5.17(\mathrm{~d}, J=3.8 \mathrm{~Hz}, 1 \mathrm{H}), 5.23(\mathrm{~d}, J=3.8 \mathrm{~Hz}, 1 \mathrm{H}), 5.25(\mathrm{~d}, J=3.2 \mathrm{~Hz}$, 1H), $5.56(\mathrm{~d}, J=3.2 \mathrm{~Hz}, 1 \mathrm{H}) ;{ }^{1} \mathrm{H}$ NMR $\left(500 \mathrm{MHz}, \mathrm{CDCl}_{3}, 1 \mathrm{D}-\mathrm{TOCSY}\right.$, irradiation at $\mathrm{H} 1^{\mathrm{I}}, 5.56$ ppm): $\delta 3.45\left(\mathrm{dd}, J=9.6,2.9 \mathrm{~Hz}, 1 \mathrm{H}, \mathrm{H} 2^{\mathrm{I}}\right), 3.96\left(\mathrm{dd}, J=9.0,9.0 \mathrm{~Hz}, 1 \mathrm{H}, \mathrm{H} 3^{\mathrm{I}}\right), 4.18(\mathrm{~d}, J=8.8 \mathrm{~Hz}$, 1H, $\left.4^{4}\right) ;{ }^{1} \mathrm{H}$ NMR (500 MHz, $\mathrm{CDCl}_{3}, 1 \mathrm{D}-\mathrm{TOCSY}$, irradiation at $\left.\mathrm{H} 6{ }^{\mathrm{II}}, 4.45 \mathrm{ppm}\right): \delta 3.19$ (dd, $J=$ 9.7, 3.4 Hz, 1H, H2 $\left.2^{\mathrm{II}}\right), 3.57\left(\mathrm{dd}, J=9.3,9.3 \mathrm{~Hz}, 1 \mathrm{H}, \mathrm{H} 3^{\mathrm{II}}\right), 3.71\left(\mathrm{dd}, J=9.5,9.5 \mathrm{~Hz}, 1 \mathrm{H}, \mathrm{H} 4^{\mathrm{II}}\right), 3.99$ (m, $\left.1 \mathrm{H}, \mathrm{H} 5^{\mathrm{II}}\right), 4.30\left(\mathrm{dd}, J=12.4,3.8 \mathrm{~Hz}, 1 \mathrm{H}, \mathrm{H} 6{ }^{\mathrm{II}}\right), 5.12\left(\mathrm{~d}, J=3.0 \mathrm{~Hz}, 1 \mathrm{H}, \mathrm{H} 1^{\mathrm{II}}\right) ;{ }^{13} \mathrm{C} \mathrm{NMR}(125.7$ $\left.\mathrm{MHz}, \mathrm{CDCl}_{3}\right): \delta 20.85\left(\mathrm{CH}_{3}\right), 58.16\left(\mathrm{CH}_{3}\right), 58.22\left(\mathrm{CH}_{3}\right), 58.28\left(\mathrm{CH}_{3}\right), 58.42\left(\mathrm{CH}_{3}\right), 58.62\left(\mathrm{CH}_{3}\right)$, $58.79\left(\mathrm{CH}_{3}\right), 58.97\left(2 \times \mathrm{CH}_{3}\right), 59.02\left(\mathrm{CH}_{3}\right), 59.06\left(\mathrm{CH}_{3}\right), 59.13\left(\mathrm{CH}_{3}\right), 59.18\left(\mathrm{CH}_{3}\right), 60.53\left(\mathrm{CH}_{3}\right)$, 61.22 $\left(\mathrm{CH}_{3}\right), 61.23\left(\mathrm{CH}_{3}\right), 61.27\left(\mathrm{CH}_{3}\right), 61.43\left(\mathrm{CH}_{3}\right), 61.47\left(2 \times \mathrm{CH}_{3}\right), 62.87\left(\mathrm{CH}_{2}\right), 68.67(\mathrm{CH})$, $70.77\left(\mathrm{CH}_{2}\right), 70.77(\mathrm{CH}), 70.88(\mathrm{CH}), 70.90(\mathrm{CH}), 70.92(\mathrm{CH}), 70.99\left(\mathrm{CH}_{2}\right), 71.12(\mathrm{CH}), 71.14$ $\left(\mathrm{CH}_{2}\right), 71.21\left(\mathrm{CH}_{2}\right), 71.41\left(\mathrm{CH}_{2}\right), 78.45(\mathrm{CH}), 78.63(\mathrm{CH}), 78.80(\mathrm{CH}), 79.29(\mathrm{CH}), 79.98(\mathrm{CH})$, $80.03(\mathrm{CH}), 80.19(\mathrm{CH}), 80.39(\mathrm{CH}), 80.77(\mathrm{CH}), 81.31(\mathrm{CH}), 81.46(\mathrm{CH}), 81.71(3 \times \mathrm{CH}), 81.80$ $(\mathrm{CH}), 81.97(\mathrm{CH}), 82.14(\mathrm{CH}), 82.19(\mathrm{CH}), 82.27(2 \times \mathrm{CH}), 82.38(\mathrm{CH}), 97.91(\mathrm{CH}), 98.11(\mathrm{CH})$, 
$98.64(\mathrm{CH}), 99.02(\mathrm{CH}), 99.11(\mathrm{CH}), 99.22(\mathrm{CH}), 99.60(\mathrm{CH}), 168.56(\mathrm{C}), 170.41(\mathrm{C})$; MS (ESI TOF): $m / z(\%) 1449\left[(\mathrm{M}+\mathrm{Na})^{+}, 100\right] ; \mathrm{HRMS}\left(\mathrm{ESI}^{+}-\mathrm{TOF}\right): m / z[\mathrm{M}+\mathrm{Na}]^{+}$calcd for $\mathrm{C}_{62} \mathrm{H}_{106} \mathrm{NaO}_{36}$ 1449.6362; found 1449.6410. Anal. calcd for $\mathrm{C}_{62} \mathrm{H}_{106} \mathrm{O}_{36}$ : C, 52.17; H, 7.48. Found: C, 52.46; H, 7.40.

Oxidative HAT of $2^{I-V I}, 3^{I-V I}, 6^{I I-V I}$-hexadeca-O-methyl- $\alpha$-cyclomaltohexaose (36). A solution of alcohol $36^{35}$ (150 mg, $\left.0.125 \mathrm{mmol}\right)$ in dry $\mathrm{CH}_{2} \mathrm{Cl}_{2}(5 \mathrm{~mL})$ containing DIB (88 $\left.\mathrm{mg}, 0.27 \mathrm{mmol}\right)$ and $\mathrm{I}_{2}(32 \mathrm{mg}, 0.125 \mathrm{mmol})$ was stirred under nitrogen at $28{ }^{\circ} \mathrm{C}$ for $1.5 \mathrm{~h}$ while irradiated with two 80 W tungsten-filament lamps. The reaction mixture was then directly loaded onto a silica gel (TLC Silica gel $60 \mathrm{~F}_{254}$, scraped from Merck Aluminum sheets) column chromatography (hexanes-acetone, $65: 35 \rightarrow 55: 45)$ to give the ten-membered lactone $37(23 \mathrm{mg}, 0.019 \mathrm{mmol}, 16 \%)$, cyclo- $5^{\mathrm{VI}}, 6^{\mathrm{I}}$ anhydro- $\left(5^{\mathrm{VI}} R\right)-(2,3,6$-tri- $O$-methyl- $\alpha$-D-xylo-hexos-5-ulopyranosyl)-[(1 $\rightarrow 4)-2,3$-di- $O$-methyl- $\alpha$ D-glucopyranosyl $]_{2}-[(1 \rightarrow 4)-2,3,6 \text {-tri- } O \text {-methyl- } \alpha \text {-D-glucopyranosyl }]_{3}(\mathbf{3 8})(37 \mathrm{mg}, 0.031 \mathrm{mmol}$, 25\%), and the lactone-alcohol 39 (14.6 mg, $0.012 \mathrm{mmol}, 10 \%)$. Compound 37: colorless oil, $[\alpha]_{\mathrm{D}}$ +131.7 (c 1.31, $\mathrm{CHCl}_{3}$ ); IR (film): 2929, 1760, 1745, 1454, 1367, 1107, $1046 \mathrm{~cm}^{-1} ;{ }^{1} \mathrm{H}$ NMR (500 $\left.\mathrm{MHz}_{\mathrm{CDCl}}\right): \delta 2.12(\mathrm{~s}, 3 \mathrm{H}), 3.09(\mathrm{dd}, J=9.9,3.3 \mathrm{~Hz}, 1 \mathrm{H}), 3.12(\mathrm{dd}, J=10.1,3.2 \mathrm{~Hz}, 1 \mathrm{H}), 3.17$ (dd, $J=9.8,3.5 \mathrm{~Hz}, 2 \mathrm{H}), 3.20(\mathrm{dd}, J=10.1,3.5 \mathrm{~Hz}, 1 \mathrm{H}), 3.36(\mathrm{~s}, 3 \mathrm{H}), 3.36(\mathrm{~s}, 3 \mathrm{H}), 3.38(\mathrm{~s}, 3 \mathrm{H})$, $3.38(\mathrm{~s}, 3 \mathrm{H}), 3.44(\mathrm{~s}, 3 \mathrm{H}), 3.48(\mathrm{~s}, 3 \mathrm{H}), 3.49(\mathrm{~s}, 3 \mathrm{H}), 3.50(\mathrm{~s}, 3 \mathrm{H}), 3.52(\mathrm{~s}, 6 \mathrm{H}), 3.55(\mathrm{~s}, 3 \mathrm{H}), 3.59$ (s, 3H), $3.60(\mathrm{~s}, 3 \mathrm{H}), 3.60(\mathrm{~s}, 3 \mathrm{H}), 3.70(\mathrm{~s}, 3 \mathrm{H}), 3.74(\mathrm{~s}, 3 \mathrm{H}), 3.87(\mathrm{dd}, J=11.0,3.8 \mathrm{~Hz}, 1 \mathrm{H}), 3.92$ $(\mathrm{dd}, J=10.7,10.7 \mathrm{~Hz}, 1 \mathrm{H}), 3.94(\mathrm{dd}, J=10.4,3.2 \mathrm{~Hz}, 1 \mathrm{H}), 4.09(\mathrm{~d}, J=5.7 \mathrm{~Hz}, 1 \mathrm{H}), 4.33(\mathrm{ddd}, J$ $=10.1,4.1,1.6 \mathrm{~Hz}, 1 \mathrm{H}), 4.48(\mathrm{dd}, J=10.7,5.4 \mathrm{~Hz}, 1 \mathrm{H}), 4.61(\mathrm{ddd}, J=10.1,10.1,5.4 \mathrm{~Hz}, 1 \mathrm{H})$, $4.95(\mathrm{~d}, J=3.5 \mathrm{~Hz}, 2 \mathrm{H}), 5.02(\mathrm{~d}, J=3.5 \mathrm{~Hz}, 1 \mathrm{H}), 5.07(\mathrm{~d}, J=3.2 \mathrm{~Hz}, 2 \mathrm{H}), 6.37(\mathrm{~d}, J=1.3 \mathrm{~Hz}$, 1H); ${ }^{1} \mathrm{H}$ NMR (500 MHz, $\mathrm{CDCl}_{3}, 1 \mathrm{D}-\mathrm{TOCSY}$, irradiation at $\left.\mathrm{H}^{\mathrm{I}}, 6.37 \mathrm{ppm}\right): \delta 3.59(\mathrm{~d}, J=7.7 \mathrm{~Hz}$, 1H, H2 $\left.2^{\mathrm{I}}\right), 3.73\left(\mathrm{~m}, 1 \mathrm{H}, \mathrm{H} 3^{\mathrm{I}}\right), 4.09\left(\mathrm{~d}, J=5.8 \mathrm{~Hz}, 1 \mathrm{H}, \mathrm{H} 4^{\mathrm{I}}\right) ;{ }^{1} \mathrm{H}$ NMR $\left(500 \mathrm{MHz}, \mathrm{CDCl}_{3}, 1 \mathrm{D}-\right.$ 
TOCSY, irradiation at $\left.\mathrm{H} 5^{\mathrm{II}}, 4.61 \mathrm{ppm}\right): \delta 3.08\left(\mathrm{dd}, J=9.9,3.3 \mathrm{~Hz}, 1 \mathrm{H}, \mathrm{H} 2^{\mathrm{II}}\right), 3.44(\mathrm{dd}, J=9.1,9.1$ $\left.\mathrm{Hz}, 1 \mathrm{H}, \mathrm{H} 3^{\mathrm{II}}\right), 3.59\left(\mathrm{dd}, J=9.6,9.6 \mathrm{~Hz}, 1 \mathrm{H}, \mathrm{H} 4^{\mathrm{II}}\right), 3.91\left(\mathrm{dd}, J=10.6,10.6 \mathrm{~Hz}, 1 \mathrm{H}, \mathrm{H} 6^{\mathrm{II}}\right), 4.48(\mathrm{dd}$, $\left.J=10.8,5.3 \mathrm{~Hz}, 1 \mathrm{H}, \mathrm{H} 6{ }^{\mathrm{II}}\right), 4.94\left(\mathrm{~d}, J=2.5 \mathrm{~Hz}, 1 \mathrm{H}, \mathrm{H} 1^{\mathrm{II}}\right) ;{ }^{1} \mathrm{H} \mathrm{NMR}\left(500 \mathrm{MHz}, \mathrm{CDCl}_{3}, 1 \mathrm{D}-\mathrm{TOCSY}\right.$, irradiation at $\left.\mathrm{H}^{\mathrm{VI}}, 4.33 \mathrm{ppm}\right): \delta 3.19\left(\mathrm{dd}, J=9.9,2.9 \mathrm{~Hz}, 1 \mathrm{H}, \mathrm{H} 2^{\mathrm{VI}}\right), 3.49(\mathrm{dd}, J=9.6,9.6 \mathrm{~Hz}, 1 \mathrm{H}$, $\left.\mathrm{H} 4^{\mathrm{VI}}\right), 3.68\left(\mathrm{dd}, J=9.6,9.6 \mathrm{~Hz}, 1 \mathrm{H}, \mathrm{H} 3^{\mathrm{VI}}\right), 4.94\left(\mathrm{~d}, J=2.7 \mathrm{~Hz}, 1 \mathrm{H}, \mathrm{H} 1^{\mathrm{VI}}\right) ;{ }^{1} \mathrm{H}$ NMR $(500 \mathrm{MHz}$, $\mathrm{CDCl}_{3}, 1 \mathrm{D}-\mathrm{ROESY}$, irradiation at $\left.\mathrm{H} 1^{\mathrm{I}}, 6.37 \mathrm{ppm}\right): \delta 3.59\left(\mathrm{dd}, J=9.3,9.3 \mathrm{~Hz}, 1 \mathrm{H}, \mathrm{H} 4^{\mathrm{II}}\right), 4.09$ (d, $\left.J=5.5 \mathrm{~Hz}, 1 \mathrm{H}, \mathrm{H} 4^{\mathrm{I}}\right) ;{ }^{1} \mathrm{H} \mathrm{NMR}\left(500 \mathrm{MHz}, \mathrm{CDCl}_{3}, 1 \mathrm{D}-\mathrm{ROESY}\right.$, irradiation at $\left.\mathrm{H} 4^{\mathrm{I}}, 4.09 \mathrm{ppm}\right): \delta$ $4.95\left(\mathrm{~d}, J=3.5 \mathrm{~Hz}, 1 \mathrm{H}, \mathrm{H} 1{ }^{\mathrm{VI}}\right) ;{ }^{13} \mathrm{C} \mathrm{NMR}\left(125.7 \mathrm{MHz}, \mathrm{CDCl}_{3}\right): \delta 21.48\left(\mathrm{CH}_{3}\right), 57.71\left(\mathrm{CH}_{3}\right), 57.76$ $\left(\mathrm{CH}_{3}\right), 58.02\left(\mathrm{CH}_{3}\right), 58.25\left(\mathrm{CH}_{3}\right), 58.33\left(\mathrm{CH}_{3}\right), 58.56\left(\mathrm{CH}_{3}\right), 58.89\left(\mathrm{CH}_{3}\right), 58.91\left(\mathrm{CH}_{3}\right), 58.99$ $\left(\mathrm{CH}_{3}\right), 60.13\left(\mathrm{CH}_{3}\right), 60.68\left(\mathrm{CH}_{3}\right), 61.30\left(\mathrm{CH}_{3}\right), 61.40\left(\mathrm{CH}_{3}\right), 61.66\left(\mathrm{CH}_{3}\right), 61.99\left(\mathrm{CH}_{3}\right), 62.10$ $\left(\mathrm{CH}_{3}\right), 62.60(\mathrm{CH}), 66.24\left(\mathrm{CH}_{2}\right), 70.79(\mathrm{CH}), 70.82(\mathrm{CH}), 71.07\left(\mathrm{CH}_{2}\right), 71.17(\mathrm{CH}), 71.35(2 \times$ $\left.\mathrm{CH}_{2}\right), 71.39(\mathrm{CH}), 71.51\left(\mathrm{CH}_{2}\right), 79.60(\mathrm{CH}), 81.04(\mathrm{CH}), 81.11(2 \times \mathrm{CH}), 81.14(\mathrm{CH}), 81.26(\mathrm{CH})$, $81.40(\mathrm{CH}), 81.43(\mathrm{CH}), 81.52(2 \times \mathrm{CH}), 81.65(\mathrm{CH}), 81.83(2 \times \mathrm{CH}), 81.90(\mathrm{CH}), 81.96(2 \times \mathrm{CH})$, $82.33(\mathrm{CH}), 83.00(\mathrm{CH}), 94.66(\mathrm{CH}), 98.56(\mathrm{CH}), 98.90(\mathrm{CH}), 99.53(\mathrm{CH}), 99.64(\mathrm{CH}), 99.80$ (CH), $170.02(\mathrm{C}), 170.23(\mathrm{C})$; MS (ESI $\left.{ }^{+}-\mathrm{TOF}\right): \mathrm{m} / z(\%) 1245\left[(\mathrm{M}+\mathrm{Na})^{+}, 100\right]$; HRMS (ESI ${ }^{+}-$ TOF): $\mathrm{m} / z[\mathrm{M}+\mathrm{Na}]^{+}$calcd for $\mathrm{C}_{53} \mathrm{H}_{90} \mathrm{NaO}_{31}$ 1245.5364; found 1245.5369. Anal. calcd for $\mathrm{C}_{53} \mathrm{H}_{90} \mathrm{O}_{31}: \mathrm{C}, 52.04 ; \mathrm{H}, 7.42$. Found: $\mathrm{C}, 52.15 ; \mathrm{H}, 7.33$. Compound 38: colorless oil, $[\alpha]_{D}+134.3$ (c 1.32, $\mathrm{CHCl}_{3}$ ); IR (film): 3475, 2929, 1454, 1367, 1139, 1107, $1046 \mathrm{~cm}^{-1} ;{ }^{1} \mathrm{H}$ NMR (500 MHz, $\left.\mathrm{CDCl}_{3}\right): \delta 3.10-3.17(\mathrm{~m}, 4 \mathrm{H}), 3.19(\mathrm{dd}, J=9.9,3.3 \mathrm{~Hz}, 1 \mathrm{H}), 3.25(\mathrm{dd}, J=9.8,3.2 \mathrm{~Hz}, 1 \mathrm{H}), 3.35$ (s, 3H), 3.36 (s, 3H), 3.369 (s, 3H), 3.372 (s, 3H), 3.46 (s, 6H), 3.48 (s, 3H), 3.49 (s, 3H), 3.50 (s, 3H), $3.51(\mathrm{~s}, 3 \mathrm{H}), 3.58(\mathrm{~s}, 3 \mathrm{H}), 3.60(\mathrm{~s}, 3 \mathrm{H}), 3.62(\mathrm{~s}, 3 \mathrm{H}), 3.64(\mathrm{~s}, 3 \mathrm{H}), 3.70(\mathrm{~s}, 3 \mathrm{H}), 3.73(\mathrm{~s}, 3 \mathrm{H})$, $5.00(\mathrm{~d}, J=3.8 \mathrm{~Hz}, 1 \mathrm{H}), 5.01(\mathrm{~d}, J=3.8 \mathrm{~Hz}, 1 \mathrm{H}), 5.025(\mathrm{~d}, J=3.8 \mathrm{~Hz}, 1 \mathrm{H}), 5.033(\mathrm{~d}, J=4.1 \mathrm{~Hz}$, $1 \mathrm{H}), 5.06(\mathrm{~d}, J=3.2 \mathrm{~Hz}, 1 \mathrm{H}), 5.18(\mathrm{~d}, J=3.5 \mathrm{~Hz}, 1 \mathrm{H}) ;{ }^{13} \mathrm{C} \mathrm{NMR}\left(125.7 \mathrm{MHz}, \mathrm{CDCl}_{3}\right): \delta 57.66$ 
$\left(\mathrm{CH}_{3}\right), 57.82\left(\mathrm{CH}_{3}\right), 57.94\left(2 \times \mathrm{CH}_{3}\right), 58.34\left(\mathrm{CH}_{3}\right), 58.51\left(\mathrm{CH}_{3}\right), 58.74\left(\mathrm{CH}_{3}\right), 58.79\left(\mathrm{CH}_{3}\right), 59.07$ $\left(\mathrm{CH}_{3}\right), 59.14\left(\mathrm{CH}_{3}\right), 61.26\left(\mathrm{CH}_{3}\right), 61.44\left(\mathrm{CH}_{3}\right), 61.52\left(\mathrm{CH}_{3}\right), 61.86\left(\mathrm{CH}_{2}\right), 61.93\left(\mathrm{CH}_{3}\right), 61.98$ $\left(\mathrm{CH}_{3}\right), 62.24\left(\mathrm{CH}_{3}\right), 63.80\left(\mathrm{CH}_{2}\right), 66.28(\mathrm{CH}), 70.90(\mathrm{CH}), 70.93(\mathrm{CH}), 71.24\left(\mathrm{CH}_{2}\right), 71.57(\mathrm{CH})$, $71.80\left(\mathrm{CH}_{2}\right), 71.82\left(\mathrm{CH}_{2}\right), 71.94\left(\mathrm{CH}_{2}\right), 72.04(\mathrm{CH}), 78.29(\mathrm{CH}), 80.35(\mathrm{CH}), 80.56(\mathrm{CH}), 80.74$ $(\mathrm{CH}), 80.96(\mathrm{CH}), 81.03(\mathrm{CH}), 81.07(\mathrm{CH}), 81.26(\mathrm{CH}), 81.40(2 \times \mathrm{CH}), 81.72(\mathrm{CH}), 81.96(\mathrm{CH})$, $82.01(2 \times \mathrm{CH}), 82.23(\mathrm{CH}), 82.31(\mathrm{CH}), 82.59(\mathrm{CH}), 82.72(\mathrm{CH}), 97.35(\mathrm{CH}), 97.79(\mathrm{CH}), 98.77$ (CH), $99.62(\mathrm{CH}), 99.90(\mathrm{CH}), 100.07(\mathrm{CH}), 100.81(\mathrm{C})$; MS (ESI $\left.{ }^{+}-\mathrm{TOF}\right): \mathrm{m} / \mathrm{z}(\%) 1217[(\mathrm{M}+$ $\left.\mathrm{Na})^{+}, 100\right]$; HRMS (ESI - TOF): $\mathrm{m} / z[\mathrm{M}+\mathrm{Na}]^{+}$calcd for $\mathrm{C}_{52} \mathrm{H}_{90} \mathrm{NaO}_{30}$ 1217.5415; found 1217.5419. Anal. calcd for $\mathrm{C}_{52} \mathrm{H}_{90} \mathrm{O}_{30}$ : C, 52.25; H, 7.59. Found: C, 52.43; H, 7.61. Compound 39: colorless oil, $[\alpha]_{\mathrm{D}}+111.4\left(\right.$ c $\left.0.96, \mathrm{CHCl}_{3}\right)$; IR (film): 3468, 2929, 1757, 1454, 1367, 1137, 1109, $1044 \mathrm{~cm}^{-1} ;{ }^{1} \mathrm{H}$ NMR (500 MHz, $\left.\mathrm{CDCl}_{3}\right): \delta 3.13-3.20(\mathrm{~m}, 3 \mathrm{H}), 3.23(\mathrm{dd}, J=9.8,3.5 \mathrm{~Hz}, 1 \mathrm{H}), 3.38$ (s, 3H), 3.385 (s, 6H), 3.39 (s, 3H), 3.48 (s, 6H), 3.49 (s, 3H), 3.50 (s, 3H), 3.51 (s, 3H), 3.58 (s, $3 \mathrm{H}), 3.63(\mathrm{~s}, 6 \mathrm{H}), 3.64(\mathrm{~s}, 3 \mathrm{H}), 3.65(\mathrm{~s}, 3 \mathrm{H}), 3.66(\mathrm{~s}, 3 \mathrm{H}), 3.69(\mathrm{~s}, 3 \mathrm{H}), 3.87(\mathrm{dd}, J=8.5,8.5 \mathrm{~Hz}$, 1H), $4.03(\mathrm{~d}, J=8.5 \mathrm{~Hz}, 1 \mathrm{H}), 4.08-4.15(\mathrm{~m}, 1 \mathrm{H}), 4.24(\mathrm{ddd}, J=10.1,2.8,1.6 \mathrm{~Hz}, 1 \mathrm{H}), 5.06(\mathrm{~d}, J$ $=3.5 \mathrm{~Hz}, 2 \mathrm{H}), 5.065(\mathrm{~d}, J=3.8 \mathrm{~Hz}, 1 \mathrm{H}), 5.12(\mathrm{~d}, J=3.5 \mathrm{~Hz}, 1 \mathrm{H}), 5.16(\mathrm{~d}, J=3.5 \mathrm{~Hz}, 1 \mathrm{H}), 5.47$ (d, $J=2.8 \mathrm{~Hz}, 1 \mathrm{H}) ;{ }^{1} \mathrm{H}$ NMR (500 MHz, $\mathrm{CDCl}_{3}, 1 \mathrm{D}-\mathrm{TOCSY}$, irradiation at $\mathrm{H}^{\mathrm{I}}, 5.47 \mathrm{ppm}$ ): $\delta 3.45$ $\left(\mathrm{dd}, J=10.0,2.7 \mathrm{~Hz}, 1 \mathrm{H}, \mathrm{H} 2^{\mathrm{I}}\right), 3.88\left(\mathrm{dd}, J=9.3,9.3 \mathrm{~Hz}, 1 \mathrm{H}, \mathrm{H} 3^{\mathrm{I}}\right), 4.03\left(\mathrm{~d}, J=8.7 \mathrm{~Hz}, 1 \mathrm{H}, \mathrm{H} 4^{\mathrm{I}}\right)$; ${ }^{1} \mathrm{H}$ NMR (500 MHz, $\mathrm{CDCl}_{3}, 1 \mathrm{D}-\mathrm{TOCSY}$, irradiation at $\mathrm{H}^{\mathrm{VI}}, 4.24 \mathrm{ppm}$ ): $\delta 3.24$ (dd, $J=9.7,3.6$ $\mathrm{Hz}, 1 \mathrm{H}, \mathrm{H} 2^{\mathrm{VI}}$ ), $3.60\left(\mathrm{dd}, J=10.0,9.4 \mathrm{~Hz}, 1 \mathrm{H}, \mathrm{H} 4^{\mathrm{VI}}\right), 3.69\left(\mathrm{dd}, J=9.4,9.4 \mathrm{~Hz}, 1 \mathrm{H}, \mathrm{H} 3^{\mathrm{VI}}\right), 3.89(\mathrm{dd}$, $\left.J=10.7,3.1 \mathrm{~Hz}, 1 \mathrm{H}, \mathrm{H}^{\mathrm{VI}}\right), 5.17\left(\mathrm{~d}, J=2.9 \mathrm{~Hz}, 1 \mathrm{H}, \mathrm{H} 1^{\mathrm{VI}}\right) ;{ }^{1} \mathrm{H} \mathrm{NMR}\left(500 \mathrm{MHz}, \mathrm{CDCl}_{3}, 1 \mathrm{D}-\right.$ ROESY, irradiation at $\left.\mathrm{H}^{\mathrm{I}}, 4.03 \mathrm{ppm}\right): \delta 5.16\left(\mathrm{~d}, J=3.4 \mathrm{~Hz}, 1 \mathrm{H}, \mathrm{H} 1^{\mathrm{VI}}\right) ;{ }^{13} \mathrm{C} \mathrm{NMR}(125.7 \mathrm{MHz}$, $\left.\mathrm{CDCl}_{3}\right): \delta 57.91\left(4 \times \mathrm{CH}_{3}\right), 58.35\left(\mathrm{CH}_{3}\right), 58.91\left(2 \times \mathrm{CH}_{3}\right), 58.98\left(2 \times \mathrm{CH}_{3}\right), 59.14\left(\mathrm{CH}_{3}\right), 61.24$ $\left(\mathrm{CH}_{3}\right), 61.56\left(\mathrm{CH}_{3}\right), 61.56\left(\mathrm{CH}_{2}\right), 61.62\left(\mathrm{CH}_{3}\right), 61.65\left(\mathrm{CH}_{3}\right), 61.75\left(2 \times \mathrm{CH}_{3}\right), 71.08(2 \times \mathrm{CH})$, 
$71.08\left(\mathrm{CH}_{2}\right), 71.13(\mathrm{CH}), 71.21\left(\mathrm{CH}_{2}\right), 71.25\left(\mathrm{CH}_{2}\right), 71.34\left(\mathrm{CH}_{2}\right), 71.36(\mathrm{CH}), 71.65(\mathrm{CH}), 80.02$ $(\mathrm{CH}), 80.13(\mathrm{CH}), 80.29(\mathrm{CH}), 81.20(\mathrm{CH}), 81.23(\mathrm{CH}), 81.26(\mathrm{CH}), 81.34(\mathrm{CH}), 81.51(\mathrm{CH})$, $81.78(\mathrm{CH}), 81.84(2 \times \mathrm{CH}), 81.89(\mathrm{CH}), 81.97(\mathrm{CH}), 82.01(\mathrm{CH}), 82.11(2 \times \mathrm{CH}), 82.16(\mathrm{CH})$, $82.32(\mathrm{CH}), 99.26(\mathrm{CH}), 99.39(\mathrm{CH}), 99.68(\mathrm{CH}), 99.74(\mathrm{CH}), 100.34(\mathrm{CH}), 100.37(\mathrm{CH}), 169.42$ (C); MS (ESI -TOF): $m / z(\%) 1203$ [(M + Na) $\left.{ }^{+}, 100\right]$ HRMS (ESI ${ }^{+}$-TOF): $m / z[\mathrm{M}+\mathrm{Na}]^{+}$calcd for $\mathrm{C}_{51} \mathrm{H}_{88} \mathrm{NaO}_{30}$ 1203.5258; found 1203.5254. Anal. calcd for $\mathrm{C}_{51} \mathrm{H}_{88} \mathrm{O}_{30}$ : C, 51.86; H, 7.51. Found: C, 51.74; H, 7.40.

Methyl 6-O-tert-Butyldiphenylsilyl-2,3,4-tri-O-methyl- $\alpha$-D-glucopyranosyl-(1 $\rightarrow 4)$-6-O-tertbutyldiphenylsilyl-2,3-di-O-methyl- $\beta$-D-glucopyranoside (40 $\beta$ ) and Methyl 6-O-tertButyldiphenylsilyl-2,3,4-tri-O-methyl- $\alpha$-D-glucopyranosyl-(1 $\rightarrow 4)$-6-O-tert-butyldiphenylsilyl2,3-di-O-methyl- $\alpha$-D-glucopyranoside $(40 \alpha)$. To a solution of dry D-(+)-maltose (5 g, $14.6 \mathrm{mmol})$, imidazole (8.9 g, $131.6 \mathrm{mmol})$, and DMAP $(8.9 \mathrm{~g}, 73.1 \mathrm{mmol})$ in dry DMF $(75 \mathrm{~mL})$ was added TBDPSCl $(22.8 \mathrm{~mL}, 87.7 \mathrm{mmol})$ at $0{ }^{\circ} \mathrm{C}$. The mixture was stirred at room temperature for $20 \mathrm{~h}$, poured into water, and extracted with $\mathrm{CH}_{2} \mathrm{Cl}_{2}$. The organic phase was washed with $\mathrm{HCl}(10 \%)$, saturated aqueous $\mathrm{NaHCO}_{3}$, and concentrated under reduced pressure to give a residue that was dried over $\mathrm{P}_{2} \mathrm{O}_{5}$ in a high vacuum desiccator for $24 \mathrm{~h}$ and used in the subsequent reaction as a mixture without further purification. $\mathrm{NaH}(60 \%, 7 \mathrm{~g}, 175 \mathrm{mmol})$ was added in portions to a solution of the crude residue $(32 \mathrm{~g})$ in dry DMF I $\mathrm{mL}$ ) cooled to $0{ }^{\circ} \mathrm{C}$ and the mixture stirred at this temperature for $1 \mathrm{~h}$. MeI $(13.7 \mathrm{~mL}, 219.3 \mathrm{mmol})$ was then added dropwise and the stirring continued for $3 \mathrm{~h}$ at room temperature. The excess of $\mathrm{NaH}$ was destroyed with $\mathrm{MeOH}$ and the mixture poured into water, extracted with $\mathrm{CH}_{2} \mathrm{Cl}_{2}$ and concentrated under reduced pressure. The residue was purified by silica gel column chromatography (hexanes-EtOAc, 9:1 $\rightarrow 8: 2$ ) and only the disilylated anomeric isomers $\mathbf{4 0 \beta}(4.3 \mathrm{~g}, 4.76 \mathrm{mmol}, 33 \%)$ and $\mathbf{4 0} \alpha(1.6 \mathrm{~g}, 1.78 \mathrm{mmol}, 12 \%)$ 
were studied. Compound 40ß: $R_{\mathrm{f}}=0.49\left(n\right.$-hexane-EtOAc, 7:3), colorless oil, $[\alpha]_{\mathrm{D}}+44.5(c 1.19$, $\mathrm{CHCl}_{3}$ ); IR (film): 2933, 1148, $1104 \mathrm{~cm}^{-1} ;{ }^{1} \mathrm{H} \mathrm{NMR}\left(500 \mathrm{MHz}, \mathrm{CDCl}_{3}\right): \delta 0.94$ (s, 9H), 1.00 (s, 9H), $3.04(\mathrm{dd}, J=9.1,7.9 \mathrm{~Hz}, 1 \mathrm{H}), 3.12(\mathrm{dd}, J=9.6,3.9 \mathrm{~Hz}, 1 \mathrm{H}), 3.31(\mathrm{~m}, 1 \mathrm{H}), 3.39(\mathrm{~m}, 3 \mathrm{H}), 3.45$ (dd, $J=8.8,8.8 \mathrm{~Hz}, 1 \mathrm{H}), 3.48(\mathrm{~m}, 2 \mathrm{H}), 3.53(\mathrm{~s}, 3 \mathrm{H}), 3.53(\mathrm{~s}, 3 \mathrm{H}), 3.57(\mathrm{~s}, 3 \mathrm{H}), 3.59(\mathrm{~s}, 3 \mathrm{H}), 3.60$ (s, 3H), 3.64 (s, 3H), $3.66(\mathrm{dd}, J=9.8,8.8 \mathrm{~Hz}, 1 \mathrm{H}), 3.74(\mathrm{dd}, J=11.0,5.7 \mathrm{~Hz}, 1 \mathrm{H}), 3.85(\mathrm{dd}, J=$ 11.0, 2.2 Hz, 1H), $4.17(\mathrm{~d}, J=7.6 \mathrm{~Hz}, 1 \mathrm{H}), 5.55(\mathrm{~d}, J=4.1 \mathrm{~Hz}, 1 \mathrm{H}), 7.15-7.66(\mathrm{~m}, 20 \mathrm{H}){ }^{1}{ }^{\mathrm{H}} \mathrm{NMR}$ $\left(500 \mathrm{MHz}, \mathrm{CDCl}_{3}, 1 \mathrm{D}-\mathrm{TOCSY}\right.$, irradiation at $\left.\mathrm{H}^{\mathrm{I}}, 4.17 \mathrm{ppm}\right): \delta 3.04\left(\mathrm{dd}, J=8.8,8.1 \mathrm{~Hz}, 1 \mathrm{H}, \mathrm{H} 2^{\mathrm{I}}\right)$, $3.41\left(\mathrm{~m}, 1 \mathrm{H}, \mathrm{H} 5^{\mathrm{I}}\right), 4.45\left(\mathrm{dd}, J=8.9,8.9 \mathrm{~Hz}, 1 \mathrm{H}, \mathrm{H} 3^{\mathrm{I}}\right), 3.66\left(\mathrm{dd}, J=9.3,9.3 \mathrm{~Hz}, 1 \mathrm{H}, \mathrm{H} 4^{\mathrm{I}}\right), 3.74(\mathrm{dd}$, $\left.J=11.2,5.9 \mathrm{~Hz}, 1 \mathrm{H}, \mathrm{H6a}^{\mathrm{I}}\right), 3.84\left(\mathrm{dd}, J=10.9,2.3 \mathrm{~Hz}, 1 \mathrm{H}, \mathrm{H}^{\mathrm{I}} \mathrm{b}^{\mathrm{I}}\right) ;{ }^{1} \mathrm{H} \mathrm{NMR}\left(500 \mathrm{MHz}, \mathrm{CDCl}_{3}\right.$, 1D-TOCSY, irradiation at $\left.\mathrm{H} 1^{\mathrm{II}}, 5.55 \mathrm{ppm}\right): \delta 3.11\left(\mathrm{dd}, J=9.3,3.4 \mathrm{~Hz}, 1 \mathrm{H}, \mathrm{H} 2^{\mathrm{II}}\right), 3.31(\mathrm{~m}, 1 \mathrm{H}$, $\left.\mathrm{H} 5^{\mathrm{II}}\right), 3.37\left(\mathrm{~m}, 2 \mathrm{H}, \mathrm{H} 3^{\mathrm{II}}\right.$ and $\left.\mathrm{H} 4^{\mathrm{II}}\right), 3.46\left(\mathrm{br} \mathrm{d}, J=11.1 \mathrm{~Hz}, 1 \mathrm{H}, \mathrm{H} 6 \mathrm{a}^{\mathrm{II}}\right), 3.52(\mathrm{br} \mathrm{d}, J=11.3 \mathrm{~Hz}, 1 \mathrm{H}$, $\left.\mathrm{H}_{6} \mathrm{~b}^{\mathrm{II}}\right) ;{ }^{13} \mathrm{C} \mathrm{NMR}\left(125.7 \mathrm{MHz}, \mathrm{CDCl}_{3}\right): \delta 19.23(\mathrm{C}), 19.31(\mathrm{C}), 26.79\left(3 \times \mathrm{CH}_{3}\right), 26.81\left(3 \times \mathrm{CH}_{3}\right)$, $56.67\left(\mathrm{CH}_{3}\right), 59.15\left(\mathrm{CH}_{3}\right), 59.58\left(\mathrm{CH}_{3}\right), 60.16\left(\mathrm{CH}_{3}\right), 60.18\left(\mathrm{CH}_{3}\right), 60.71\left(\mathrm{CH}_{3}\right), 62.14\left(\mathrm{CH}_{2}\right), 63.61$ $\left(\mathrm{CH}_{2}\right), 71.89(\mathrm{CH}), 72.06(\mathrm{CH}), 75.11(\mathrm{CH}), 78.84(\mathrm{CH}), 81.90(\mathrm{CH}), 83.38(\mathrm{CH}), 83.77(\mathrm{CH})$, $86.41(\mathrm{CH}), 96.12(\mathrm{CH}), 104.04(\mathrm{CH}), 127.43(2 \times \mathrm{CH}), 127.47(2 \times \mathrm{CH}), 127.49(2 \times \mathrm{CH}), 127.54$ $(2 \times \mathrm{CH}), 129.36(\mathrm{CH}), 129.43(\mathrm{CH}), 129.47(2 \times \mathrm{CH}), 133.27(\mathrm{C}), 133.34(\mathrm{C}), 133.72(\mathrm{C}), 133.86$ (C), $135.38(2 \times \mathrm{CH}), 135.56(2 \times \mathrm{CH}), 135.77(4 \times \mathrm{CH}) ; \mathrm{MS}\left(\mathrm{ESI}^{+}-\mathrm{TOF}\right): m / z(\%) 925[(\mathrm{M}+$ $\left.\mathrm{Na})^{+}, 100\right]$; HRMS (ESI ${ }^{+}-\mathrm{TOF}$ ): $m / z[\mathrm{M}+\mathrm{Na}]^{+}$calcd for $\mathrm{C}_{50} \mathrm{H}_{70} \mathrm{NaO}_{11} \mathrm{Si}_{2}$ 925.4354; found 925.4366. Anal. calcd for $\mathrm{C}_{50} \mathrm{H}_{70} \mathrm{O}_{11} \mathrm{Si}_{2}: \mathrm{C}, 66.49 ; \mathrm{H}, 7.81$. Found: $\mathrm{C}, 66.75 ; \mathrm{H}, 7.93$. Compound

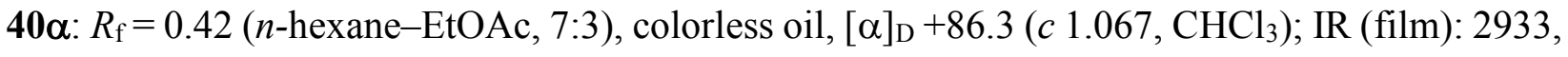
1156, 1104, $1029 \mathrm{~cm}^{-1} ;{ }^{1} \mathrm{H}$ NMR (500 MHz, $\left.\mathrm{CDCl}_{3}\right): \delta 0.94$ (s, 9H), 0.99 (s, 9H), 3.11 (dd, $J=$ 9.8, 4.1 Hz, 1H), 3.17 (dd, $J=9.6,3.6 \mathrm{~Hz}, 1 \mathrm{H}), 3.36(\mathrm{~s}, 3 \mathrm{H}), 3.51$ (s, $6 \mathrm{H}), 3.56(\mathrm{~s}, 3 \mathrm{H}), 3.60$ (s, 3H), $3.63(\mathrm{~s}, 3 \mathrm{H}), 4.78(\mathrm{~d}, J=3.5 \mathrm{~Hz}, 1 \mathrm{H}), 5.55(\mathrm{~d}, J=4.1 \mathrm{~Hz}, 1 \mathrm{H}), 7.16-7.66(\mathrm{~m}, 20 \mathrm{H}) ;{ }^{13} \mathrm{C}$ NMR 
(125.7 MHz, $\left.\mathrm{CDCl}_{3}\right): \delta 19.24(\mathrm{C}), 19.30(\mathrm{C}), 26.81\left(6 \times \mathrm{CH}_{3}\right), 54.59\left(\mathrm{CH}_{3}\right), 58.69\left(\mathrm{CH}_{3}\right), 59.52$ $\left(\mathrm{CH}_{3}\right), 60.00\left(\mathrm{CH}_{3}\right), 60.14\left(\mathrm{CH}_{3}\right), 60.70\left(\mathrm{CH}_{3}\right), 62.22\left(\mathrm{CH}_{2}\right), 63.63\left(\mathrm{CH}_{2}\right), 70.66(\mathrm{CH}), 71.92(\mathrm{CH})$, $72.48(\mathrm{CH}), 78.86(\mathrm{CH}), 81.93(\mathrm{CH}), 82.43(\mathrm{CH}), 83.37(\mathrm{CH}), 83.52(\mathrm{CH}), 96.27(\mathrm{CH}), 96.50$ $(\mathrm{CH}), 127.42(2 \times \mathrm{CH}), 127.44(2 \times \mathrm{CH}), 127.46(2 \times \mathrm{CH}), 127.53(2 \times \mathrm{CH}), 129.35(\mathrm{CH}), 129.39$ $(\mathrm{CH}), 129.43(\mathrm{CH}), 129.45(\mathrm{CH}), 133.38(\mathrm{C}), 133.41(\mathrm{C}), 133.77(\mathrm{C}), 133.88(\mathrm{C}), 135.48(2 \times \mathrm{CH})$, $135.60(2 \times \mathrm{CH}), 135.72(2 \times \mathrm{CH}), 135.80(2 \times \mathrm{CH}) ; \mathrm{MS}_{\left(\mathrm{ESI}^{+}-\mathrm{TOF}\right): m / z(\%)} 925\left[(\mathrm{M}+\mathrm{Na})^{+}\right.$, 100]; HRMS (ESI ${ }^{+}-\mathrm{TOF}$ ): $m / z$ [M + Na] ${ }^{+}$calcd for $\mathrm{C}_{50} \mathrm{H}_{70} \mathrm{NaO}_{11} \mathrm{Si}_{2}$ 925.4354; found 925.4358. Anal. calcd for $\mathrm{C}_{50} \mathrm{H}_{70} \mathrm{O}_{11} \mathrm{Si}_{2}: \mathrm{C}, 66.49 ; \mathrm{H}, 7.81$. Found: C, 66.10; H, 7.92.

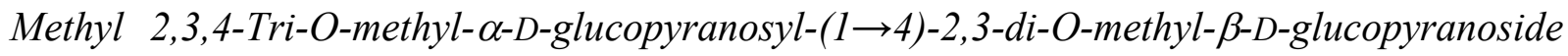
(41). To a solution of $\mathbf{4 0 \beta}(2 \mathrm{~g}, 2.2 \mathrm{mmol})$ in THF $(50 \mathrm{~mL})$ was added TBAF (1 M in THF, $6.6 \mathrm{~mL}$, $6.6 \mathrm{mmol}$ ) and the mixture stirred at room temperature for $7 \mathrm{~h}$. The THF was the removed under vacuum and the resulting oil purified by column chromatography (hexanes-EtOAc, $3: 7 \rightarrow$ EtOAc) to give the compound $41(675 \mathrm{mg}, 1.58 \mathrm{mmol}, 72 \%)$ as a colorless oil; $[\alpha]_{\mathrm{D}}+74.1\left(c 1.077, \mathrm{CHCl}_{3}\right)$; IR (film): 3460, 2936, 1144, 1081, $1029 \mathrm{~cm}^{-1} ;{ }^{1} \mathrm{H}$ NMR (500 MHz, $\left.\mathrm{CDCl}_{3}\right): \delta 3.00$ (dd, $J=9.8$, $8.8 \mathrm{~Hz}, 1 \mathrm{H}), 3.03(\mathrm{dd}, J=9.1,7.9 \mathrm{~Hz}, 1 \mathrm{H}), 3.15(\mathrm{dd}, J=9.8,4.1 \mathrm{~Hz}, 1 \mathrm{H}), 3.35$ (ddd, $J=9.8,2.8$, $2.8 \mathrm{~Hz}, 1 \mathrm{H}), 3.43$ (dd, $J=10.4,9.1 \mathrm{~Hz}, 1 \mathrm{H}), 3.43$ (dd, $J=8.8,8.8 \mathrm{~Hz}, 1 \mathrm{H}), 3.52$ (s, 3H), 3.53 (s, $3 \mathrm{H}), 3.55(\mathrm{~s}, 3 \mathrm{H}), 3.57(\mathrm{~s}, 3 \mathrm{H}), 3.58(\mathrm{~s}, 3 \mathrm{H}), 3.60(\mathrm{~s}, 3 \mathrm{H}), 3.63(\mathrm{~s}, 3 \mathrm{H}), 3.65(\mathrm{dd}, J=11.4,6.0 \mathrm{~Hz}$, 1H), $3.81(\mathrm{dd}, J=12.3,2.8 \mathrm{~Hz}, 1 \mathrm{H}), 3.85(\mathrm{dd}, J=12.3,3.2 \mathrm{~Hz}, 1 \mathrm{H}), 3.85(\mathrm{dd}, J=11.7,2.2 \mathrm{~Hz}$, $1 \mathrm{H}), 3.88(\mathrm{dd}, J=9.5,9.5 \mathrm{~Hz}, 1 \mathrm{H}), 4.19(\mathrm{~d}, J=7.9 \mathrm{~Hz}, 1 \mathrm{H}), 5.61(\mathrm{~d}, J=4.1 \mathrm{~Hz}, 1 \mathrm{H}) ;{ }^{13} \mathrm{C} \mathrm{NMR}$ (125.7 MHz, $\left.\mathrm{CDCl}_{3}\right): \delta 57.0\left(\mathrm{CH}_{3}\right), 59.9\left(\mathrm{CH}_{3}\right), 60.1\left(\mathrm{CH}_{3}\right), 60.2\left(\mathrm{CH}_{3}\right), 60.6\left(\mathrm{CH}_{3}\right), 60.8\left(\mathrm{CH}_{3}\right)$, $60.9\left(\mathrm{CH}_{2}\right), 61.9\left(\mathrm{CH}_{2}\right), 71.3(\mathrm{CH}), 72.2(\mathrm{CH}), 74.2(\mathrm{CH}), 80.5(\mathrm{CH}), 81.8(\mathrm{CH}), 83.5(\mathrm{CH}), 84.3$ (CH), $86.5(\mathrm{CH}), 96.5(\mathrm{CH}), 104.3(\mathrm{CH})$; MS (ESI $\left.{ }^{+}-\mathrm{TOF}\right): \mathrm{m} / z(\%) 449\left[(\mathrm{M}+\mathrm{Na})^{+}, 100\right]$; HRMS 
(ESI ${ }^{+}$-TOF): $m / z[\mathrm{M}+\mathrm{Na}]^{+}$calcd for $\mathrm{C}_{18} \mathrm{H}_{34} \mathrm{NaO}_{11}$ 449.1999; found 449.1994. Anal. calcd for $\mathrm{C}_{18} \mathrm{H}_{34} \mathrm{O}_{11}: \mathrm{C}, 50.70 ; \mathrm{H}, 8.04$. Found: $\mathrm{C}, 50.35 ; \mathrm{H}, 8.24$.

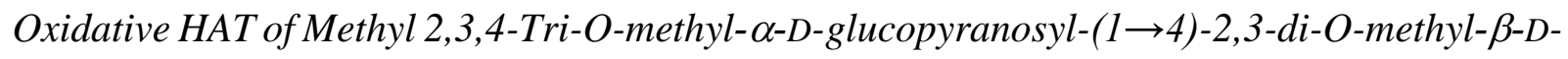
glucopyranoside (41). A solution of alcohol 41 (100 mg, $0.235 \mathrm{mmol})$ in dry $\mathrm{CH}_{2} \mathrm{Cl}_{2}(9.4 \mathrm{~mL})$ containing DIB (166.5 mg, $0.516 \mathrm{mmol})$ and $\mathrm{I}_{2}(60 \mathrm{mg}, 0.235 \mathrm{mmol})$ was stirred under nitrogen at $32{ }^{\circ} \mathrm{C}$ for $3 \mathrm{~h}$ while irradiated with two $80 \mathrm{~W}$ tungsten-filament lamps. The reaction mixture was then directly loaded onto a silica gel (TLC Silica gel $60 \mathrm{~F}_{254}$, scraped from Merck Aluminum sheets) column chromatography (hexanes-acetone, $60: 40 \rightarrow 25: 75$ ) to give the ten-membered lactone $\mathbf{4 2}$ (11 mg, $0.024 \mathrm{mmol}, 9 \%)$ and the acetyl-derivative 43 (11 mg, $0.023 \mathrm{mmol}, 10 \%)$. Compound $\mathbf{4 2}$ : colorless oil, $[\alpha]_{\mathrm{D}}-24.2$ (c 0.520, $\mathrm{CHCl}_{3}$ ); IR (film): 2938, 1746, 1455, 1372, 1126, $1095 \mathrm{~cm}^{-1} ;{ }^{1} \mathrm{H}$ $\operatorname{NMR}\left(500 \mathrm{MHz}, \mathrm{CDCl}_{3}\right): \delta 2.13(\mathrm{~s}, 3 \mathrm{H}), 2.89\left(\mathrm{dd}, J=9.3,7.7 \mathrm{~Hz}, 1 \mathrm{H}, \mathrm{H} 2^{\mathrm{II}}\right), 3.15(\mathrm{dd}, J=8.8,8.8$ $\left.\mathrm{Hz}, 1 \mathrm{H}, \mathrm{H} 3^{\mathrm{II}}\right), 3.31$ (dd, $\left.J=7.7,2.0 \mathrm{~Hz}, 1 \mathrm{H}, \mathrm{H}^{\mathrm{I}}\right), 3.45$ (s, 3H), 3.49 (s, 3H), 3.53 (s, 3H), 3.56 (dd, $\left.J=8.4,8.4 \mathrm{~Hz}, 1 \mathrm{H}, \mathrm{H} 3^{\mathrm{I}}\right), 3.59(\mathrm{~s}, 3 \mathrm{H}), 3.60(\mathrm{~s}, 3 \mathrm{H}), 3.61(\mathrm{~s}, 3 \mathrm{H}), 3.62(\mathrm{dd}, J=9.1,9.1 \mathrm{~Hz}, 1 \mathrm{H}$, $\left.\mathrm{H} 4^{\mathrm{II}}\right), 3.75\left(\mathrm{~d}, J=8.8 \mathrm{~Hz}, 1 \mathrm{H}, \mathrm{H} 4^{\mathrm{I}}\right), 3.80\left(\mathrm{ddd}, J=9.8,9.8,5.4 \mathrm{~Hz}, 1 \mathrm{H}, \mathrm{H} 5^{\mathrm{II}}\right), 4.12(\mathrm{~d}, J=7.9 \mathrm{~Hz}$, 1H, H1 $\left.{ }^{\mathrm{II}}\right), 4.18\left(\mathrm{dd}, J=11.4,9.8 \mathrm{~Hz}, 1 \mathrm{H}, \mathrm{H} 6^{\mathrm{II}}\right), 4.59\left(\mathrm{dd}, J=11.2,5.2 \mathrm{~Hz}, 1 \mathrm{H}, \mathrm{H} 6^{\mathrm{II}}\right), 6.23(\mathrm{~d}, J=$ $\left.2.2 \mathrm{~Hz}, 1 \mathrm{H}, \mathrm{H} 1^{\mathrm{I}}\right) ;{ }^{1} \mathrm{H}$ NMR $\left(500 \mathrm{MHz}, \mathrm{CDCl}_{3}, 1 \mathrm{D}-\mathrm{TOCSY}\right.$, irradiation at $\left.\mathrm{H} 1^{\mathrm{I}}, 6.23 \mathrm{ppm}\right): \delta 3.31$ $\left(\mathrm{dd}, J=7.8,1.9 \mathrm{~Hz}, 1 \mathrm{H}, \mathrm{H} 2^{\mathrm{I}}\right), 3.56\left(\mathrm{dd}, J=8.4,8.4 \mathrm{~Hz}, 1 \mathrm{H}, \mathrm{H} 3^{\mathrm{I}}\right), 3.76\left(\mathrm{~d}, J=8.7 \mathrm{~Hz}, 1 \mathrm{H}, \mathrm{H} 4^{\mathrm{I}}\right) ;{ }^{1} \mathrm{H}$ NMR (500 MHz, $\mathrm{CDCl}_{3}, 1 \mathrm{D}-\mathrm{TOCSY}$, irradiation at $\left.\mathrm{H}^{\mathrm{II}}, 2.89 \mathrm{ppm}\right): \delta 3.15$ (dd, $J=8.9,8.9 \mathrm{~Hz}$, $\left.1 \mathrm{H}, \mathrm{H} 3^{\mathrm{II}}\right), 3.63\left(\mathrm{dd}, J=9.2,9.2 \mathrm{~Hz}, 1 \mathrm{H}, \mathrm{H} 4^{\mathrm{II}}\right), 3.81\left(\mathrm{ddd}, J=9.3,9.3,5.4 \mathrm{~Hz}, 1 \mathrm{H}, \mathrm{H} 5^{\mathrm{II}}\right), 4.13(\mathrm{~d}, J$ $\left.=7.8 \mathrm{~Hz}, 1 \mathrm{H}, \mathrm{H} 1^{\mathrm{II}}\right), 4.19\left(\mathrm{dd}, J=10.6,10.6 \mathrm{~Hz}, 1 \mathrm{H}, \mathrm{H} 6^{\mathrm{II}}\right), 4.60\left(\mathrm{dd}, J=11.0,5.2 \mathrm{~Hz}, 1 \mathrm{H}, \mathrm{H} 6^{\mathrm{II}}\right)$; ${ }^{13} \mathrm{C}$ NMR (125.7 MHz, $\left.\mathrm{CDCl}_{3}\right): \delta 21.34\left(\mathrm{CH}_{3}\right), 57.08\left(\mathrm{CH}_{3}\right), 59.00\left(\mathrm{CH}_{3}\right), 60.43\left(\mathrm{CH}_{3}\right), 61.64(2$ $\left.\times \mathrm{CH}_{3}\right), 62.00\left(\mathrm{CH}_{3}\right), 65.14\left(\mathrm{CH}_{2}, \mathrm{C}^{\mathrm{II}}\right), 67.32\left(\mathrm{CH}, \mathrm{C}^{\mathrm{II}}\right), 81.51\left(\mathrm{CH}, \mathrm{C} 4^{\mathrm{I}}\right), 82.37\left(\mathrm{CH}, \mathrm{C}^{\mathrm{II}}\right), 83.04$ $\left(\mathrm{CH}, \mathrm{C} 3^{\mathrm{I}}\right), 83.22\left(\mathrm{CH}, \mathrm{C}^{\mathrm{II}}\right), 83.88\left(\mathrm{CH}, \mathrm{C}^{\mathrm{I}}\right), 84.71\left(\mathrm{CH}, \mathrm{C}^{\mathrm{II}}\right), 94.51\left(\mathrm{CH}, \mathrm{C} 1^{\mathrm{I}}\right), 103.82(\mathrm{CH}$, 
$\left.\mathrm{C} 1^{\mathrm{II}}\right), 170.32(\mathrm{C}), 170.60(\mathrm{C})$; MS (ESI $\left.{ }^{+}-\mathrm{TOF}\right): \mathrm{m} / z(\%) 475\left[(\mathrm{M}+\mathrm{Na})^{+}, 100\right]$; HRMS (ESI $\left.{ }^{+}-\mathrm{TOF}\right)$ : $m / z[\mathrm{M}+\mathrm{Na}]^{+}$calcd for $\mathrm{C}_{19} \mathrm{H}_{32} \mathrm{NaO}_{12}$ 475.1791; found 475.1793. Anal. calcd for $\mathrm{C}_{19} \mathrm{H}_{32} \mathrm{O}_{12}: \mathrm{C}$, 50.44; H, 7.13. Found: C, 50.16; H, 7.21. Compound 43: colorless oil, $[\alpha]_{\mathrm{D}}+43.8\left(c 0.820, \mathrm{CHCl}_{3}\right)$; IR (film): 3520, 2933, 1745, 1456, 1369, 1107, 1081, $1055 \mathrm{~cm}^{-1} ;{ }^{1} \mathrm{H}$ NMR (500 MHz, $\left.\mathrm{CDCl}_{3}\right): \delta$ $2.05(\mathrm{~s}, 3 \mathrm{H}), 3.05\left(\mathrm{dd}, J=9.1,7.6 \mathrm{~Hz}, 1 \mathrm{H}, \mathrm{H} 2^{\mathrm{II}}\right), 3.29\left(\mathrm{ddd}, J=9.8,3.0,3.0 \mathrm{~Hz}, 1 \mathrm{H}, \mathrm{H} 5^{\mathrm{II}}\right), 3.34$ (dd, $\left.J=9.0,9.0 \mathrm{~Hz}, 1 \mathrm{H}, \mathrm{H} 3^{\mathrm{II}}\right), 3.47(\mathrm{~s}, 3 \mathrm{H}), 3.52(\mathrm{~s}, 3 \mathrm{H}), 3.56(\mathrm{~s}, 3 \mathrm{H}), 3.58(\mathrm{~s}, 3 \mathrm{H}), 3.61(\mathrm{~s}, 3 \mathrm{H})$, $3.78\left(\mathrm{dd}, J=9.5,9.5 \mathrm{~Hz}, 1 \mathrm{H}, \mathrm{H} 4^{\mathrm{II}}\right), 3.86\left(\mathrm{dd}, J=12.3,2.8 \mathrm{~Hz}, 1 \mathrm{H}, \mathrm{H} 6^{\mathrm{II}}\right), 3.94(\mathrm{~d}, J=12.0 \mathrm{~Hz}, 1 \mathrm{H}$, H6 $\left.{ }^{\mathrm{I}}\right), 4.09\left(\mathrm{dd}, J=12.3,3.2 \mathrm{~Hz}, 1 \mathrm{H}, \mathrm{H} 6^{\mathrm{II}}\right), 4.09\left(\mathrm{~d}, J=1.6 \mathrm{~Hz}, 1 \mathrm{H}, \mathrm{H} 4^{\mathrm{I}}\right), 4.18(\mathrm{~d}, J=7.6 \mathrm{~Hz}, 1 \mathrm{H}$, $\left.\mathrm{H} 1^{\mathrm{II}}\right), 4.59\left(\mathrm{~d}, J=11.7 \mathrm{~Hz}, 1 \mathrm{H}, \mathrm{H}^{\mathrm{I}}\right), 4.69(\mathrm{~d}, J=6.3 \mathrm{~Hz}, 1 \mathrm{H}), 5.09(\mathrm{~d}, J=6.3 \mathrm{~Hz}, 1 \mathrm{H}), 5.58(\mathrm{~d}, J$ $\left.=2.5 \mathrm{~Hz}, 1 \mathrm{H}, \mathrm{H} 1^{\mathrm{I}}\right) ;{ }^{1} \mathrm{H}$ NMR $\left(500 \mathrm{MHz}, \mathrm{CDCl}_{3}, 1 \mathrm{D}-\mathrm{TOCSY}\right.$, irradiation at $\left.\mathrm{H} 2^{\mathrm{II}}, 3.05 \mathrm{ppm}\right): \delta 3.29$ (ddd, $\left.J=9.9,2.9,2.9 \mathrm{~Hz}, 1 \mathrm{H}, \mathrm{H} 5^{\mathrm{II}}\right), 3.34\left(\mathrm{dd}, J=9.0,9.0 \mathrm{~Hz}, 1 \mathrm{H}, \mathrm{H} 3^{\mathrm{II}}\right), 3.78(\mathrm{dd}, J=9.4,9.4 \mathrm{~Hz}$, 1H, H4 $\left.{ }^{\mathrm{II}}\right), 3.86\left(\mathrm{dd}, J=12.2,3.4 \mathrm{~Hz}, 1 \mathrm{H}, \mathrm{H} 6^{\mathrm{II}}\right), 4.09\left(\mathrm{dd}, J=12.0,2.4 \mathrm{~Hz}, 1 \mathrm{H}, \mathrm{H} 6^{\mathrm{II}}\right), 4.18(\mathrm{~d}, J=$ 7.6 Hz, $\left.1 \mathrm{H}, \mathrm{H} 1^{\mathrm{II}}\right) ;{ }^{1} \mathrm{H}$ NMR (500 MHz, $\mathrm{CDCl}_{3}, 1 \mathrm{D}-\mathrm{TOCSY}$, irradiation at $\left.\mathrm{H} 1^{\mathrm{I}}, 5.58 \mathrm{ppm}\right): \delta 3.62$ $\left(\mathrm{m}, 2 \mathrm{H}, \mathrm{H} 2^{\mathrm{I}}\right.$ and $\left.\mathrm{H} 3{ }^{\mathrm{I}}\right), 5.58\left(\mathrm{~d}, J=2.8 \mathrm{~Hz}, 1 \mathrm{H}, \mathrm{H} 4{ }^{\mathrm{I}}\right) ;{ }^{13} \mathrm{C} \mathrm{NMR}\left(125.7 \mathrm{MHz}, \mathrm{CDCl}_{3}\right): \delta 21.89\left(\mathrm{CH}_{3}\right)$, $56.82\left(\mathrm{CH}_{3}\right), 57.88\left(\mathrm{CH}_{3}\right), 59.83\left(\mathrm{CH}_{3}\right), 60.23\left(\mathrm{CH}_{3}\right), 60.43\left(\mathrm{CH}_{3}\right), 61.17\left(\mathrm{CH}_{2}, \mathrm{C} 6{ }^{\mathrm{II}}\right), 71.11\left(\mathrm{CH}_{2}\right.$, $\left.\mathrm{C}^{\mathrm{I}}\right), 74.44\left(\mathrm{CH}, \mathrm{C} 4^{\mathrm{I}}\right), 74.66\left(\mathrm{CH}, \mathrm{C} 5^{\mathrm{II}}\right), 75.13\left(\mathrm{CH}, \mathrm{C} 4^{\mathrm{II}}\right), 76.40\left(\mathrm{CH}, \mathrm{C}^{\mathrm{I}}\right.$ or $\left.\mathrm{C} 3^{\mathrm{I}}\right), 79.17\left(\mathrm{CH}, \mathrm{C}^{\mathrm{I}}\right.$ or $\left.\mathrm{C}^{\mathrm{I}}\right), 84.11\left(\mathrm{CH}, \mathrm{C} 2^{\mathrm{II}}\right), 86.22\left(\mathrm{CH}, \mathrm{C}^{\mathrm{II}}\right), 92.88\left(\mathrm{CH}_{2}\right), 96.45\left(\mathrm{C}, \mathrm{C}^{\mathrm{I}}\right), 97.16\left(\mathrm{CH}, \mathrm{C}^{1}\right), 104.29$ $\left(\mathrm{CH}, \mathrm{Cl}^{\mathrm{II}}\right), 168.79(\mathrm{C}) ; \mathrm{MS}\left(\mathrm{ESI}^{+}-\mathrm{TOF}\right): \mathrm{m} / z(\%) 505\left[(\mathrm{M}+\mathrm{Na})^{+}, 100\right] ; \mathrm{HRMS}\left(\mathrm{ESI}^{+}-\mathrm{TOF}\right): \mathrm{m} / \mathrm{z}$ $[\mathrm{M}+\mathrm{Na}]^{+}$calcd for $\mathrm{C}_{20} \mathrm{H}_{34} \mathrm{NaO}_{13}$ 505.1897; found 505.1905. Anal. calcd for $\mathrm{C}_{20} \mathrm{H}_{34} \mathrm{O}_{13}: \mathrm{C}, 49.79$; H, 7.10. Found: C, 49.46; H, 7.15.

\section{ASSOCIATED CONTENT}

\section{Supporting Information}


The Supporting Information is available free of charge on the ACS Publications website at DOI:

The material includes crystal structure of $\mathbf{1 5}$; copies of spectra for all new compounds; absolute energy and Cartesian coordinates of minimized structures of compounds $\mathbf{3 2}$ and $\mathbf{3 8}$ (PDF).

Crystallographic data (CIF).

\section{AUTHOR INFORMATION}

\section{Corresponding Author}

*angelesmartin@ipna.csic.es

*esuarez@ipna.csic.es

\section{Notes}

The authors declare no competing financial interest.

\section{ACKNOWLEDGMENTS}

Financial support by the Investigation Programs of the Ministerio de Economía y Competitividad (CTQ2010-18244), Fundación CajaCanarias (2015-BIO08) and the COST Action CM1201 "Biomimetic Radical Chemistry" is acknowledged. D.A.-D. thanks the Ministerio de Economía y Competitividad for a fellowship. We thank Dr. Shinya Fushinobu (University of Tokyo, http://www.ric.hi-ho.ne.jp/asfushi/) for access to the Cremer-Pople parameter calculator.

\section{REFERENCES:}


(1) For reviews on cyclodextrins and their applications, see: (a) Delbianco, M.; Bharate, P.; VarelaAramburu, S.; Seeberger, P. H. Chem. Rev. 2016, 116, 1693-1752. (b) Crini, G. Chem. Rev. 2014, 114, 10940-10975. (c) Todres, Z. V. In Organic Chemistry in Confining Media; Springer, Switzerland, 2013, chap. 1, pp. 1-63. (d) Davis, F.; Higson, S. In Macrocycles: Construction, Chemistry and Nanotechnology Applications; John Wiley \& Sons, 2011, pp. 190-254. (e) Cyclodextrins and Their Complexes; Dodziuk, H. Ed.; Wiley-VCH: Weinheim, 2006.

(2) (a) Simões, S. M. N.; Rey-Rico, A.; Concheiro, A.; Alvarez-Lorenzo, C. Chem. Commun. 2015, 51, 6275-6289. (b) Rodríguez-Lavado, J.; de la Mata, M.; Jiménez-Blanco, J. L.; García-Moreno, M. I.; Benito, J. M.; Díaz-Quintana, A.; Sánchez-Alcázar, J. A.; Higaki, K.; Nanba, E.; Ohno, K.; Suzuki, Y.; Ortiz Mellet, C.; García Fernández, J. M. Org. Biomol. Chem. 2014, 12, 2289-2301. (c) Otero-Espinar, F. J.; Blanco-Méndez, J. Curr. Top. Med. Chem. 2014, 14, 463-551. (d) Ortiz Mellet, C.; García Fernández, J. M.; Benito, J. M. Chem. Soc. Rev. 2011, 40, 1586-1608. (e) Ortiz Mellet, C.; Benito, J. M.; García Fernández, J. M. Chem. Eur. J. 2010, 16, 6728-6742. (f) LazaKnoerr, A. L.; Gref, R.; Couvreur, P. J. Drug Targeting 2010, 18, 645-656.

(3) (a) Raynal, M.; Ballester, P.; Vidal-Ferran, A.; van Leeuwen, P. W. N. M. Chem. Soc. Rev. 2014, 43, 1734-1787. (b) Pedersen, C. M.; Bols, M. In Organic Synthesis and Molecular Engineering; Nielsen, M. B. Ed.; Wiley: Hoboken, 2013; pp. 305-332. (c) Zhou, Y.; Pedersen, C. M.; Bols, M. Tetrahedron Lett. 2013, 54, 2458-2461. (d) Zhou, Y.; Marinescu, L.; Pedersen, C. M.; Bols, M. Eur. J. Org. Chem. 2012, 6383-6389. (e) Breslow, R. In Artificial Enzymes; WileyVCH: Weinheim, 2005.

(4) (a) Hapiot, F.; Bricout, H.; Menuel, S.; Tilloy, S.; Monflier, E. Catal. Sci. Technol. 2014, 4, 1899-1908. (b) Kanagaraj, K.; Pitchumani, K. J. Org. Chem. 2013, 78, 744-751, (c) Liang, L.; Diallo, A. K.; Salmon, L.; Ruiz, J.; Astruc, D. Eur. J. Inorg. Chem. 2012, 2950-2958. (d) Senra, J. 
D.; Malta, L. F. B.; Aguiar, L. C. S.; Simas, A. B. C.; Antunes, O. A. C. Curr. Org. Chem. 2010, 14, 1337-1355. (e) Hapiot, F.; Tilloy, S.; Monflier, E. Chem. Rev. 2006, 106, 767-781.

(5) For recent examples on modified cyclodextrins, see: (a) Menuel, S.; Dourmert, B.; Saitzek, S.; Ponchel, A.; Delevoye, L.; Monflier, E.; Hapiot, F. J. Org. Chem. 2015, 80, 6259-6266. (b) Gallego-Yerga, L.; González-Álvarez, M. J.; Mayordomo, N.; Santoyo-González, F.; Benito, J. M.; Ortiz Mellet, C.; Mendicuti, F.; García Fernández, J. M. Chem. Eur. J. 2014, 20, 6622-6627. (c) Balbuena, P.; Gonçalves-Pereira, R.; Jiménez Blanco, J. L.; García-Moreno, M. I.; Lesur, D.; Ortiz Mellet, C.; García Fernández, J. M. J. Org. Chem. 2013, 78, 1390-1403. (d) Xiao, S.; Yang, M.; Yu, F.; Zhang, L.; Zhou, D.; Sinaÿ, P.; Zhang, Y. Tetrahedron 2013, 69, 4053-4060. (e) Zhang, P.; Wing, A.; Cui, L.; Ling, C.-C. Org. Lett. 2012, 14, 1612-1615. (f) Ghosh, R.; Zhang, P.; Wang, A.; Ling, C.-C. Angew. Chem., Int. Ed. 2012, 51, 1548-1552. For a review, see: (g) Guieu, S.; Sollogoub, M. In Modern Synthetic Methods in Carbohydrate Chemistry: From Monosaccharides to Complex Glycoconjugates; Werz, D. B.; Vidal, S. Eds.; Wiley-VCH: Weinheim, 2013, pp. 241283.

(6) (a) Prochowicz, D.; Kornowicz, A.; Justyniak, I.; Lewiński, J. Coord. Chem. Rev. 2016, 306, 331-345. (b) Harada, A.; Takashima, Y.; Nakahata, M. Acc. Chem. Res. 2014, 47, 2128-2140. (c) Zerkoune, L.; Angelova, A.; Lesieur, S. Nanomaterials 2014, 4, 741-765. (d) Gallego-Yerga, L.; Lomazzi, M.; Sansone, F.; Ortiz Mellet, C.; Casnati, A.; García Fernández, J. M. Chem. Commun. 2014, 50, 7440-7443. (e) Engeldinger, E.; Armspach, D.; Matt, D. Chem. Rev. 2003, 103, 41474173.

(7) (a) Gattuso, G.; Nepogodiev, S. A.; Stoddart, J. F. Chem. Rev. 1998, 98, 1919-1958. (b) Asthon, P. R.; Gattuso, G.; Königer, R.; Stoddart, J. F.; Williams, D. J. J. Org. Chem. 1996, 61, 9553-9555.

(c) Yamamura, H.; Masuda, H.; Kawase, Y.; Kawai, M.; Butsugan, Y.; Einaga, H. Chem. Commun. 1996, 1069-1070. (d) Asthon, P. R.; Boyd, S. E.; Gattuso, G.; Hartwell, E. Y.; Königer, R.; 
Spencer, N.; Stoddart, J. F. J. Org. Chem. 1995, 60, 3898-3903. (e) Asthon, P. R.; Ellwood, P.; Staton, I.; Stoddart, J. F. J. Org. Chem. 1991, 56, 7274-7280. (f) Gadelle, A.; Defaye, J. Angew. Chem., Int. Ed. Engl. 1991, 30, 78-80. (g) Fujita, K.; Yamamura, H.; Imoto, T.; Tabushi, I. Chem. Lett. 1988, 17, 543-546.

(8) (a) Lindner, H. J.; Yuan, D.-Q.; Fujita, K.; Kubo, K.; Lichtenthaler, F. W. Chem. Commun. 2003, 1730-1731. (b) Fujita, K.; Chen, W.-H.; Yuan, D.-Q.; Nogami, Y.; Koga, T.; Fujioka, T.; Mihashi, K.; Immel, S.; Lichtenthaler, F. W. Tetrahedron: Asymmetry 1999, 10, 1689-1696. (c) Ikeda, H.; Nagano, Y.; Du, Y.; Ikeda, T.; Toda, F. Tetrahedron Lett. 1990, 31, 5045-5048.

(9) (a) Ohta, K.; Fujita, K.; Shimada, H.; Ikegami, Y.; Nogami, Y.; Koga, T. Chem. Phar. Bull. 1997, 45, 631-635. (b) Nogami, Y.; Nasu, K.; Koga, T.; Ohta, K.; Fujita, K.; Immel, S.; Lindner, H. J.; Schmitt, G. E.; Lichtenthaler, F. W. Angew. Chem., Int. Ed. Engl. 1997, 36, 1899-1902. (c) Fujita, K.; Shimada, H.; Ohta, K.; Nogami, Y.; Nasu, K.; Koga, T. Angew. Chem., Int. Ed. Engl. 1995, 34, 1621-1622.

(10) (a) Caira, M. R.; Griffith, V. J.; Nassimbeni, L. R.; van Oudtshoorn, B. J. Chem. Soc., Perkin Trans. 2, 1994, 2071-2072. (b) Steiner, T.; Saenger, W. Angew. Chem. Int. Ed. 1998, 37, 34043407.

(11) (a) Frihed, T. G.; Bols, M.; Pedersen, C. M. Eur. J. Org. Chem. 2016, 2740-2756. (b) Boultadakis-Arapinis, M.; Lescot, C.; Micouin, L.; Lecourt, T. Synlett 2013, 24, 2477-2491. (c) Pérez-Martín, I.; Suárez, E. In Encyclopedia of Radicals in Chemistry, Biology and Materials; Chatgilialoglu, C.; Studer, A. Eds.; Wiley: Chichester, 2012; Vol. 2, pp. 1131-1174.

(12) Gilbert, B. C.; Smith, J. R. L.; Taylor, P.; Ward, S.; Whitwood, A. C. J. Chem. Soc., Perkin Trans. 2 2000, 2001-2007.

(13) (a) Francisco, C. G.; Herrera, A. J.; Kennedy, A. R.; Martín, A.; Melián, D.; Pérez-Martín, I.; Quintanal, L. M.; Suárez, E. Chem. Eur. J. 2008, 14, 10369-10381. (b) Martín, A. Pérez-Martín, 
I.; Quintanal, L. M.; Suárez, E. Org. Lett. 2007, 9, 1785-1788. (c) Francisco, C. G.; Herrera, A. J.; Kennedy, A. R.; Melián, D.; Suárez, E. Angew. Chem. Int. Ed. 2002, 41, 856-858. For a recent review on HAT, see: (d) Nechab, M.; Mondal, S.; Bertrand, M. P. Chem. Eur. J. 2014, 20, 1603416059.

(14) Since it is generally accepted that a narrow range of distances of approximately $2.5-3.0 \AA$ are required for the abstraction to take place, the distances between the $\mathrm{O} 6$ and the extractable hydrogens are indicative of HAT reaction feasibility. (a) Feray, L.; Kuznetsov, N.; Renaud, P. In Radicals in Organic Synthesis; Renaud, P.; Sibi, M. P. Eds.; Wiley-VCH: Weinheim, 2001; Vol. 2, pp. 246-278. (b) Dorigo, A. E.; Houk, K. N. J. Am. Chem. Soc. 1987, 109, 2195-2197. (c) Dorigo, A. E.; Houk, K. N. J. Org. Chem. 1988, 53, 1650-1664. (d) Dorigo, A. E.; McCarrick, M. A.; Loncharich, R. J.; Houk, K. N. J. Am. Chem. Soc. 1990, 112, 7508-7514.

(15) Steiner, T. Saenger, W. Angew. Chem. Int. Ed. 1998, 37, 3404-3407.

(16) Alvarez-Dorta, D.; León, E. I.; Kennedy, A. R.; Martín, A.; Pérez-Martín, I.; Suárez, E. Angew. Chem. Int. Ed. 2015, 54, 3674-3678.

(17) (a) Chen, Z.; Bradshaw, J. S.; Shen, Y.-F.; Habata, Y.; Lee, M. L. J. Org. Chem. 1997, 62, 8529-8534. (b) Chen, Z.; Bradshaw, J. S.; Lee, M. L. Tetrahedron Lett. 1996, 37, 6831-6834. (c) Yi, G.; Bradshaw, J. S.; Rossiter, B. E.; Malik, A.; Li, W.; Petersson, P.; Markides, K. E.; Lee, M. L. J. Org. Chem. 1993, 58, 4844-4850. For other procedures for the synthesis of mono- and dihydroxyl permethylated CDs, see: (d) Lecourt, T.; Mallet, J.-M.; Sinaÿ, P. Carbohydr. Res. 2003, 338, 2417-2419. (e) du Roizel, B.; Baltaze, J.-P.; Sinaÿ, P. Tetrahedron Lett. 2002, 43, 2371-2373. (f) Kaneda, T.; Fujimoto, T.; Goto, J.; Asano, K.; Yasufuku, Y.; Jung, J. H.; Hosono, C.; Sakata, Y. Chem. Lett. 2002, 514-515. (g) Tanaka, M.; Kawaguchi, Y.; Niinae, T.; Shono, T. J. Chromatogr. 1984, 314, 193-200. 
(18) (a) Sabadini, E.; Egídio, F. C.; Fujiwara, F. Y.; Cosgrove, T. J. Phys. Chem. B 2008, 112, 3328-3332. (b) Kusmin, A.; Lechner, R. E.; Kammel, M.; Saenger, W. J. Phys. Chem. B 2008, $112,12888-12898$.

[19] We have found scant information on the synthesis and conformational studies of 1,3,5trioxocane compounds, see: (a) Burkert, U. Z. Naturforsch. B 1980, 35, 1479-1481. (b) Anet, F. A. L. In Conformational Analysis of Medium-Sized Heterocycles; Glass, R. S. Ed.; VCH: New York, 1988; pp. 35-95. (c) McGuire, R. R.; Pflug, J. L.; Rakowsky, M. H.; Shackelford, S. A.; Shaffer, A. A. Heterocycles 1994, 38, 1979-2004. (d) Heller, L.; Schwarz, S.; Perl, V.; Köwitsch, A.; Siewert, B.; Csuk, R. Eur. J. Med. Chem. 2015, 101, 391-399.

(20) The intermolecular addition of an external nucleophile has previously been observed by us in disaccharide systems but only as a minor side reaction, see: León, E. I.; Martín, A.; Pérez-Martín, I.; Quintanal, L. M.; Suárez, E. Eur. J. Org. Chem. 2010, 5248-5262. See others examples in Ref. 13a.

(21) Coupling constants and dihedrals were calculated with Maestro 9.9.013 on minimized structures using MacroModel and the AMBER* force field with the GB/SA solvent model for $\mathrm{CHCl}_{3}$, Schrödinger, LLC, New York, NY, 2009. The experimental constants were determined on the basis of 1D TOCSY experiments.

(22) Although, according to the IUPAC nomenclature the unit VII is more usually named as (5R)$5^{\mathrm{VII}}-O$-acetyl-2,3,6-tri- $O$-methyl- $\alpha$-D- $x y l o$-hexos-5-ulopyranosyl we prefer to see it as a 5 - $C$ acetoxy- $\beta$-L-idopyranosyl derivative, which described better the inversion at $\mathrm{C} 5^{\mathrm{VII}}$ and the ${ }^{1} C_{4}$ conformation of the pyranose ring and is also alternatively accepted by the IUPAC (see a similar example in Nomenclature of Carbohydrates, Rule 2-Carb-16.1). 
(23) For a recent review on hypervalent iodine compounds and their applications see: (a) Yoshimura, A.; Zhdankin, V. V. Chem. Rev. 2016, 116, 3328-3435. For the preparation of [Bis(acyloxy)iodo]arenes, see: (b) Varvoglis, A. In Hypervalent Iodine in Organic Synthesis; Academic Press: London, 1997; pp. 11-12. (c) Sharefkin, J. G.; Saltzman, H. Org. Synth. Coll. 1973, $V, 660-663$.

(24) Courtneidge, J. L.; Lusztyk, J.; Pagé, D. Tetrahedron Lett. 1994, 35, 1003-1006.

(25) Fenger, T. H.; Bjerre, J.; Bols, M. ChemBioChem 2009, 10, 2494-2503.

(26) Chaise, T.; Cardinael, P.; Tisse, S.; Combret, J.-C.; Bouillon, J.-P. Tetrahedron: Asymmetry 2008, 19, 348-357.

(27) Only a few reports concerning 1,7-diols for the preparation of 1,6-dioxaspiro[4.4]nonane derivatives through favorable 1,5-HAT reactions have been published, see: (a) González, A. G.; Francisco, C. G.; Freire, R.; Hernández, R.; Salazar, J. A.; Suárez, E. Tetrahedron Lett. 1976, 17, 2725-2728. (b) Concepción, J. I.; Francisco, C. G.; Hernández, R.; Salazar, J. A.; Suárez, E. Tetrahedron Lett. 1984, 25, 1953-1956. (c) Urones, J. G.; Basabe, P.; Marcos, I. S.; Martín, D. D.; Sexmero, M. J.; Peral, M. H.; Broughton, H. B. Tetrahedron 1992, 48 10389-10398. (d) Paolucci, C.; Mazzini, C.; Fava, A. J. Org. Chem. 1995, 60, 169-175.

(28) MacroModel, version 9.9.013 and the AMBER* force field with the GB/SA solvent model for $\mathrm{CHCl}_{3}$, Schrödinger, LLC, New York, NY, 2014.

(29) Kumprecht, L.; Budesinsky, M.; Bour, P.; Kraus, T. New J. Chem. 2010, 34, 2254-2260.

(30) CCDC 1039442 contains the supplementary crystallographic data for the compound $\mathbf{1 5}$. These data can be obtained free of charge from The Cambridge Crystallographic Data Centre via www.ccdc.cam.ac.uk/data_request/cif. 
(31) Steiner, T.; Saenger, W. Carbohydr. Res. 1996, 282, 53-63. Crystallographic data were obtained by courtesy of The Cambridge Crystallographic Data Centre $($ Database code CSD $=$ TEVCEC).

(32) Cremer, D.; Pople, J. A. J. Am. Chem. Soc. 1975, 97, 1354-1358.

(33) A similar situation is encountered in spiro ortho ester of disaccharide systems, see: (a) Ashworth, P.; Belagali, S. L.; Casson, S.; Marczak, A.; Kocienski, P. Tetrahedron 1991, 47, 9939_9946. (b) Ohtake, H.; Iimori, T.; Ikegami, S. Tetrahedron Lett. 1997, 38, 3413-3414. (c) Ohtake, H.; Ichiba, N.; Shiro, M.; Ikegami, S. J. Org. Chem. 2000, 65, 8164-8170. (d) Nicolaou, K. C.; Mitchell, H. J.; Fylaktakidou, K. C.; Suzuki, H.; Rodríguez, R. M. Angew. Chem. Int. Ed. 2000, 39, 1089-1093.

(34) Easy dehomologation of 5-O-yl secondary radicals has been described in furanose rings, however the homologous reaction of 6- $O$-yl radicals in pyranose systems has not been observed, see: (a) Guyenne, S.; León, E. I.; Martín, A.; Pérez-Martín, I.; Suárez, E. J. Org. Chem. 2012, 77, 7371-7391. (b) Boto, A.; Hernández, D.; Hernández, R.; Suárez, E. J. Org. Chem. 2003, 68, 5310-5319. For an analogous radical decarboxylation of aldofuranuronic acids, see: (c) Dhavale, D. D.; Tagliavini, E.; Trombini, C.; Umani-Ronchi, A. Tetrahedron Lett. 1988, 29, 6163-6166.

(35) Armspach, D.; Poorters, L.; Matt, D.; Benmerad, B.; Balegroune, F.; Toupet, L. Org. Biomol. Chem. 2005, 3, 2588-2592.

(36) Pawar, D. M.; Cain, D.; Gill, G.; Bain, A. D.; Sullivan, R. H.; Noe, E. A. J. Org. Chem. 2007, $72,25-29$.

(37) This 1,6-HAT reaction between 6- $O$-yl radicals and a methoxyl group at $\mathrm{C} 4$ to give a 1,3 dioxane ring, has been previously described by us in glucopyranosyl systems, see: Boto, A.; Hernández, D.; Hernández, R.; Suárez, E. J. Org. Chem. 2006, 71, 1938-1948. 
(38) (a) Carofiglio, T.; Cordioli, M.; Fornasier, R.; Jicsinszky, L.; Tonellato, U. Carbohydr. Res. 2004, 339, 1361-1366. For other procedures for the synthesis of per-2,3- $O$-methyl- $\beta-\mathrm{CD}$, see: (b) Fügedi, P. Carbohydr. Res. 1989, 192, 366-369. (c) Takeo, K.; Mitoh, H.; Uemura, K. Carbohydr. Res. 1989, 187, 203-221.

(39) (a) Yi, G.; Bradshaw, J. S.; Rossiter, B. E.; Reese, S. L.; Petersson, P.; Markides, K. E.; Lee, M. L. J. Org. Chem. 1993, 58, 2561-2565. For other procedures for the synthesis of per-2,3-Omethyl- $\alpha-C D$, see: (b) Poorters, L.; Armspach, D.; Matt, D. Eur. J. Org. Chem. 2003, 1377-1381. (c) Ashton, P. R.; Hartwell, E. Y.; Philp, D.; Spencer, N.; Stoddart, J. F. J. Chem. Soc., Perkin Trans. 2 1995, 1263-1277.

(40) For a solvent-dependent chair inversion equilibrium in a mono-altro- $\alpha$-CD derivative, see: (a) Yamauchi, K.; Miyawaki, A.; Takashima, Y.; Yamaguchi, H.; Harada, A. Org. Lett. 2010, 12, 1284-1286. For a study on conformation equilibria of related $\alpha$-L-iduronate residues in disaccharide derived from heparin, see: (b) Sanderson, P. N.; Huckerby, T. N.; Nieduszynski, I. A. Biochem. J. 1987, 243, 175-181. 